\title{
GOLD NANORODS AS MOLECULAR PROBES FOR LIGHT-BASED IMAGING TECHNIQUES
}

Raja Gopal Rayavarapu 
Thesis committee members:

$\begin{array}{ll}\text { prof.dr. H. Rogalla } & \text { University of Twente (chairman) } \\ \text { prof.dr. A.G. J. M. van Leeuwen } & \text { University of Twente (thesis advisor) } \\ \text { dr. S. Manohar } & \text { University of Twente (assistant advisor) } \\ \text { prof.dr. J.F.J. Engbersen } & \text { University of Twente } \\ \text { prof.dr. L.W.M.M. Terstappen } & \text { University of Twente } \\ \text { prof.dr.ir. B. Poelsema } & \text { University of Twente } \\ \text { prof.dr. A. Sturk } & \text { AMC, UvA } \\ \text { dr. W.H. de Jong } & \text { RIVM, Bilthoven }\end{array}$

The research described in this thesis was carried out at the Biomedical Photonic Imaging Group, MIRA Institute for Biomedical Technology and Technical Medicine, Faculty of Science and Technology, University of Twente, P. O. Box 217, 7500 AE Enschede, The Netherlands.

The research has been financially supported primarily by the former BMTI (presently MIRA) institute in the speerpunt program, NIMTIK: Non Invasive Molecular Tumor Imaging and Killing; and also by SenterNovem through the PRESMITT project (IPD067771) in the program IOP Photonic Devices; and by the Nederlandse Wetenschappelijk Organisatie (NWO) and Stichting Technische Wetenschappen (STW) through project TTF 6527.

Cover Design: Raja Gopal Rayavarapu \& Vishnu Vardhan Pully

Front cover page illustrates the deposition of gold nanorods on silicon wafer. The gold nanorods selfassemble by forming a ring-like structure when dried on the wafer as visualized using scanning electron microscopy (SEM). The SEM image shows the presence of nanorods and nanospheres along the boundary of dried pellet of gold nanorods solution. Back cover page of the thesis shows monodisperse gold nanorods in high concentration with no nanospheres as by-products, spread on the silicon wafer after drying and visualized using SEM.

Printed by: Wöhrmann Print Service, Zutphen, The Netherlands.

ISBN: 978-90-365-2994-5

DOI: $10.3990 / 1.9789036529945$

Copyright @ Raja Gopal Rayavarapu, 2010

All rights reserved. No part of the material protected by this copyright notice may be reproduced or utilized in any form or by any means, electronic or mechanical, including photo copying, recording or by any information storage and retrieval system, without prior permission from the author. 


\section{GOLD NANORODS AS MOLECULAR PROBES FOR LIGHT-BASED IMAGING TECHNIQUES}

\section{DISSERTATION}

to obtain

the degree of doctor at the University of Twente, on the authority of the rector magnificus, prof.dr. H. Brinksma, on account of the decision of the graduation committee, to be publicly defended on Thursday, March $25^{\text {th }}, 2010$ at 13.15 hrs.

by

Raja Gopal Rayavarapu born on October $5^{\text {th }}, 1978$ in Pentasriramapuram, India 
This dissertation has been approved by:

Promotor: $\quad$ prof.dr. A. G. J. M. van Leeuwen

Assistant promotor: dr. S. Manohar 
To my parents and brother 



\section{Table of Contents}

Chapter 1

Chapter 2

Chapter 3

Chapter 4

Chapter 5

Chapter 6

Chapter 7

Chapter 8

Summary

Samenvatting

Abbreviations

Acknowledgements

List of Publications

About the author
Introduction

1-12

Synthesis and bioconjugation of gold nanoparticles as

13-32

potential molecular probes for light-based imaging

techniques

Iodide impurities in hexadecyltrimethylammonium

33-46

bromide (CTAB) products: Lot-Lot variations and

influence on gold nanorod synthesis

Maneuvering gold deposition to tailor the tips of gold

47-60

nanorods by changing initial ascorbate-gold ion ratios

in synthesis

In vitro toxicity studies of polymer-coated gold

61-82

nanorods

Blood clearance and tissue distribution of PEGylated

and Non-PEGylated gold nanorods after intravenous

administration in rats

Antibody conjugation to gold nanorods via PEG

101-116

linkers: in vitro toxicity and cellular uptake studies in breast adenocarcinoma cell line

Conclusions and Outlook/Recommendations

117-126

127-128

129-132

133-134

135-136

137-138

139-140 

Chapter 1

\section{Introduction}

Metallic nanoparticles, with sizes typically between 1 and $1000 \mathrm{~nm}$, have been used as colloids or sols for ornamental decoration since the middle ages [1]. Currently, these nanoparticles are scientifically interesting owing to their size and shape dependent physical as well as chemical properties. Metallic nanoparticles capped by organic molecules (mercaptothiols, carboxylate and ammonium compounds) can be organized into ordered one-, two- and three-dimensional structures and these structures have potential applications in nanodevices and nanoelectronics [2]. Furthermore, owing to the plasmonic properties of noble metal nanoparticles, the synthesis and application of nanocrystalline metallic particles [5-7] is being studied. Electrochemical [8] and wet-chemical synthesis methods [9, 10, 11] are investigated to prepare nanoparticles with novel structures and shapes with a high yield and monodispersity 


\section{Gold nanoparticles}

At the present time, gold nanoparticles provoke much interest from an application point of view especially due to their unique optical properties in combination with their biocompatibility and chemical inertness $[12,13]$. The surface plasmon effect can cause intense field enhancements in gold nanoparticles, which endow these particles with strong intrinsic optical properties such as absorption, and scattering, but also enhanced fluorescence and Raman scattering signals from proximal molecules. Further, the surface chemistry of gold accommodates easy coupling of several organic molecules such as thiols, proteins, nucleic acids and polymers $[14,15,16]$ making directed interactions of functionalized particles with cells and sub-cellular entities. In this thesis, we will focus on tuning the optical properties of gold nanoparticles, with special attention to their absorption spectrum, as a molecular probe for biomedical/bio-imaging applications.

\section{Gold nanospheres}

Gold nanoparticles have been first developed in 1857 by Faraday in the colloidal form [1]. The nanospheres were prepared by the reduction of aqueous solution of chloroauric acid $\left(\mathrm{HAuCl}_{4} .3 \mathrm{H}_{2} \mathrm{O}\right)$ using phosphorus. Later in the $20^{\text {th }}$ century, various methods were reported in synthesizing gold particles in colloidal form. The synthesis using citrate reduction of gold reported by Turkevitch in $1951[17,18]$ has been the backbone for particles used in several applications in many fields of science. Still, improved parameters that influence the particle diameter and physical-optical properties have driven research in synthesis procedures. For example, thiols that have strong interaction with gold surfaces $[19,20]$ are being used for the synthesis of small gold clusters $(\sim 1-5 \mathrm{~nm})$. Gold nanoparticles can also be capped by several agents such as sodium citrate, cetyltrimethylammonium bromide (CTAB) [10, 11, 12], disulfides, polymers with mercapto and cyano functional groups and dendrimers $[21,22]$ that can make nanoparticles functionalized by coupling with proteins, peptides and drugs to aid in targeting of disease in vivo.

\section{Biomedical applications of gold nanospheres}

The optical and physico-chemical properties of gold nanospheres help in several applications for drug-delivery as well as diagnostic imaging [23]. For in vivo applications, the goal of nanoparticle delivery is to arrive at the diseased tissues after intravenous administration into the blood. The targeting of a disease site via nanoparticles can be either passive or active 
[24]. 'Passive' targeting depends on their extravasation through leaky (gaps $\approx 600 \mathrm{~nm}$ ) blood vessels in unhealthy tissue or due to the passive uptake by cells. Then, the size of the carrier plays a major role to take advantage of the enhanced permeation and retention (EPR) effect [25]. Chan et al [26] have recently shown that citrate capped gold nanospheres can be found clustered within the cell organelles when incubated with Hela cells. The uptake of the gold nanospheres by cells, however, is different for different diameters of spheres (see figure 1), which may hamper the biocompatibility and stability of gold nanospheres and thus their in vivo applications.
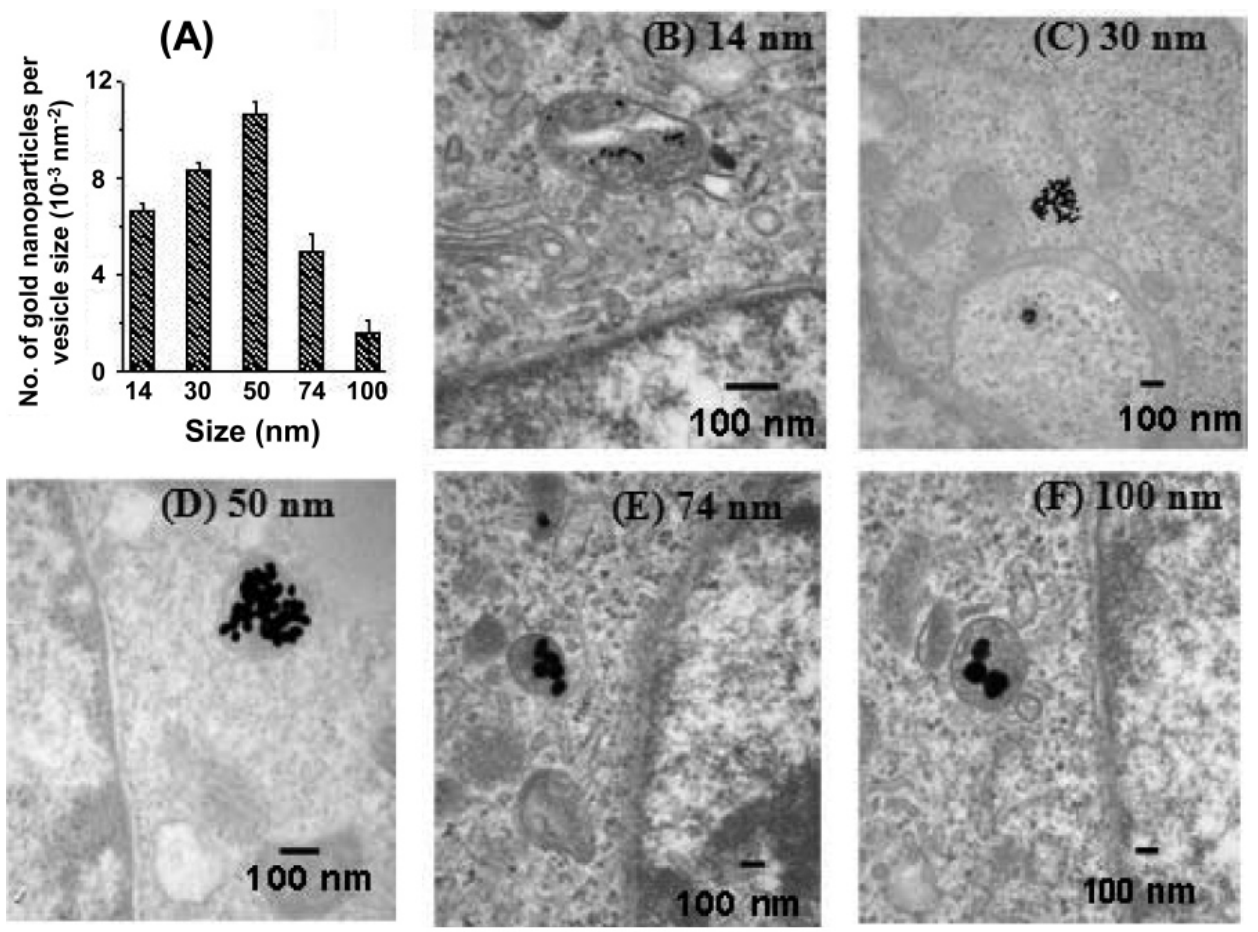

Figure 1: Transmission electron microscopy imaging and measurements of gold nanoparticles in cells. (A) The graph of number of gold nanoparticles per vesicle diameter vs. nanoparticle size. (B-F) TEM images of gold nanoparticles with sizes 14, 30,50,74, and $100 \mathrm{~nm}$ trapped inside vesicles of a Hela cell, respectively (Reproduced with permission from Ref. 26).

Active targeting of the disease site via nanoparticle conjugates (small molecules, peptides or proteins) binding to the receptors present on the cellular membrane can make the in vivo application of gold nanospheres more specific. Then, gold nanoparticles can even play a role in gene therapy via nucleic acid delivery. The effective delivery vehicles need to provide efficient cell entry, protection from nucleases that break nucleic acids and release of the nucleic acid to nucleus [27]. Rotello et al [28] have shown that gold nanoparticles, functionalized with cationic quaternary ammonium groups attach to plasmid DNA via noncovalent interaction and provide efficient gene delivery in mammalian $293 \mathrm{~T}$ cells. Liu et al 
[29] have reported the ability of gold nanoparticles to deliver plasmid DNA into breast cancer cells (MCF-7) by effective coupling of DNA by anchoring $\beta$-cyclodextrin on the periphery of oligo(ethylenediamino)-modified gold nanoparticles (OEA-CD-NP). Rotello et al [30] have also reported protein delivery via functionalized gold nanoparticles where cationic tetra-alkyl ammonium functionalized gold nanoparticles recognize the surface of an anionic protein through complementary electrostatic interaction and inhibit its activity. In a recent study, Sastry et al [31] have demonstrated functionalized gold nanoparticles as carriers of insulin that are stabilized by chitosan coated particles that adsorb insulin on their surface and provide efficient delivery for transmucosal delivery.

\section{Photothermal effects for cancer imaging and treament}

In this thesis, we focus on the use of gold nanoparticles for tumor imaging and/or eradication. Gold nanospheres have a strong absorption band between $500 \mathrm{~nm}-560 \mathrm{~nm}$ in the visible region of the electromagnetic spectrum, which results from the oscillation of free electrons due to the phenomena of surface plasmon resonance [12] (figure 2).

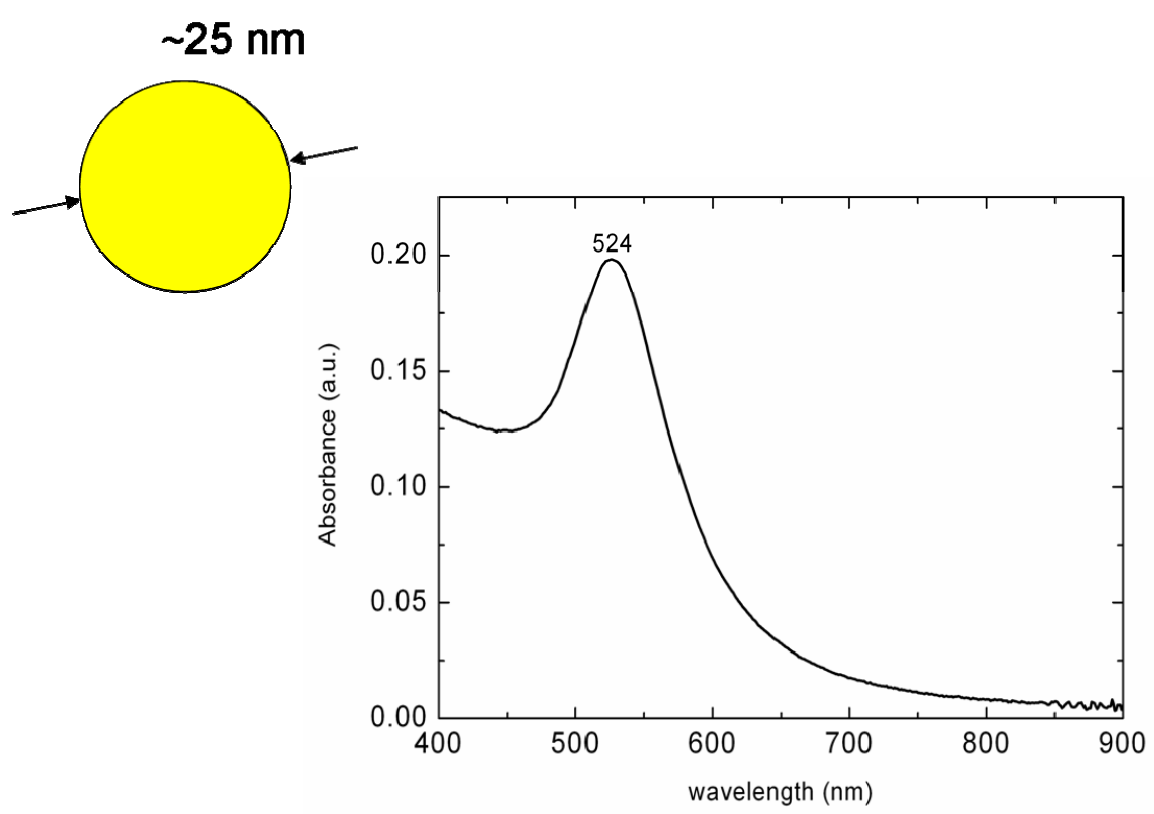

Figure 2: Gold nanospheres have a single plasmon peak absorbing light in the green region (around $524 \mathrm{~nm}$ ) of the electromagnetic spectrum.

Consequently, gold nanospheres irradiated with green light causes local heating that can produce ultrasound (US) waves (for imaging) or, with higher fluences can destroy (superficial) tumors. Citrate-stabilized gold nanospheres (core diameter, $d=30 \mathrm{~nm}$ ) coated with anti-EGFR (epidermal growth factor receptor) to target HSC3 cancer cells (human oral 
squamous cell carcinoma) in combination with light therapy indeed caused localized heating that lead to tumor cell death. Consequently, the use of gold nanospheres can enhance the efficacy of photothermal therapy by $\sim 20$ times than conventional fluorescent dyes [32]. In another approach, optically responsive delivery systems were reported by Caruso et al [33] where gold nanoparticles were incorporated into shells of microcapsules encapsulated with fluorescein labeled dextran by layer-by-layer technique [33]. The gold nanoparticles dope the capsule shells and upon irradiation with light, the shell ruptures and thus the fluorescently labeled dextran is released. Skirtach et al [34] have followed similar strategy to deliver encapsulated materials from polyelectrolyte-multilayer capsules inside living cancer cells.

\section{Limitations of using gold nanospheres}

A major limitation of gold nanospheres is that their peak light absorbance lies in the green part of the spectrum, which prevents their usage for deep in vivo imaging and treatment of diseases. The absorbance of hemoglobin compounds dominate in the visible region in comparison to the near infrared (NIR) region $(600 \mathrm{~nm}-950 \mathrm{~nm})$ of the spectrum (figure 3 ). Consequently, the penetration depth of the light in the visible region of spectrum is low [35].

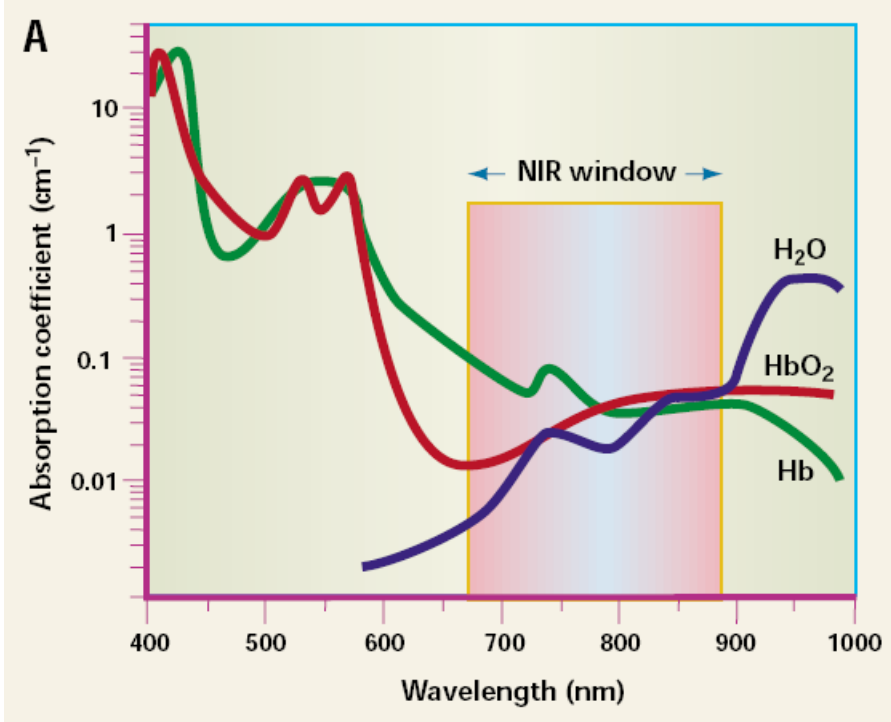

Figure 3: Absorption coefficient of reduced hemoglobin $(\mathrm{Hb})$ and Oxyhemoglobin $(\mathrm{HbO} 2)$ and water at different wavelengths. The cumulative absorption coefficient is minimal in the range of $\sim 600 \mathrm{~nm}$ to $950 \mathrm{~nm}$ that is known as the near-infrared region of the spectrum, NIR. [Reproduced with permission from Ref. 35].

Non-spherical gold nanoparticles can absorb in the NIR region, depending on the size parameters [36]. Unlike gold nanospheres, gold nanorods are characterized by two plasmon peaks. The transverse plasmon (TP) peak occurs due to plasmons excited along the short axis 
of gold nanorod causing them to absorb in the vicinity of $520 \mathrm{~nm}$. The lower energy peak, which arises from plasmons excited along the long axis of the rod, is called the longitudinal plasmon (LP) peak. The longitudinal plasmon peak can be tuned to occur in the far-red or near infrared (NIR) between $600 \mathrm{~nm}-950 \mathrm{~nm}$. The wavelength of maximal absorbance by gold nanorods depends on their aspect ratio (long axis (length)/short axis (width). Consequently, tuning these size parameters can facilitate the absorption of light from farvisible to the near infrared region (NIR) of the spectrum as shown in figure 4.

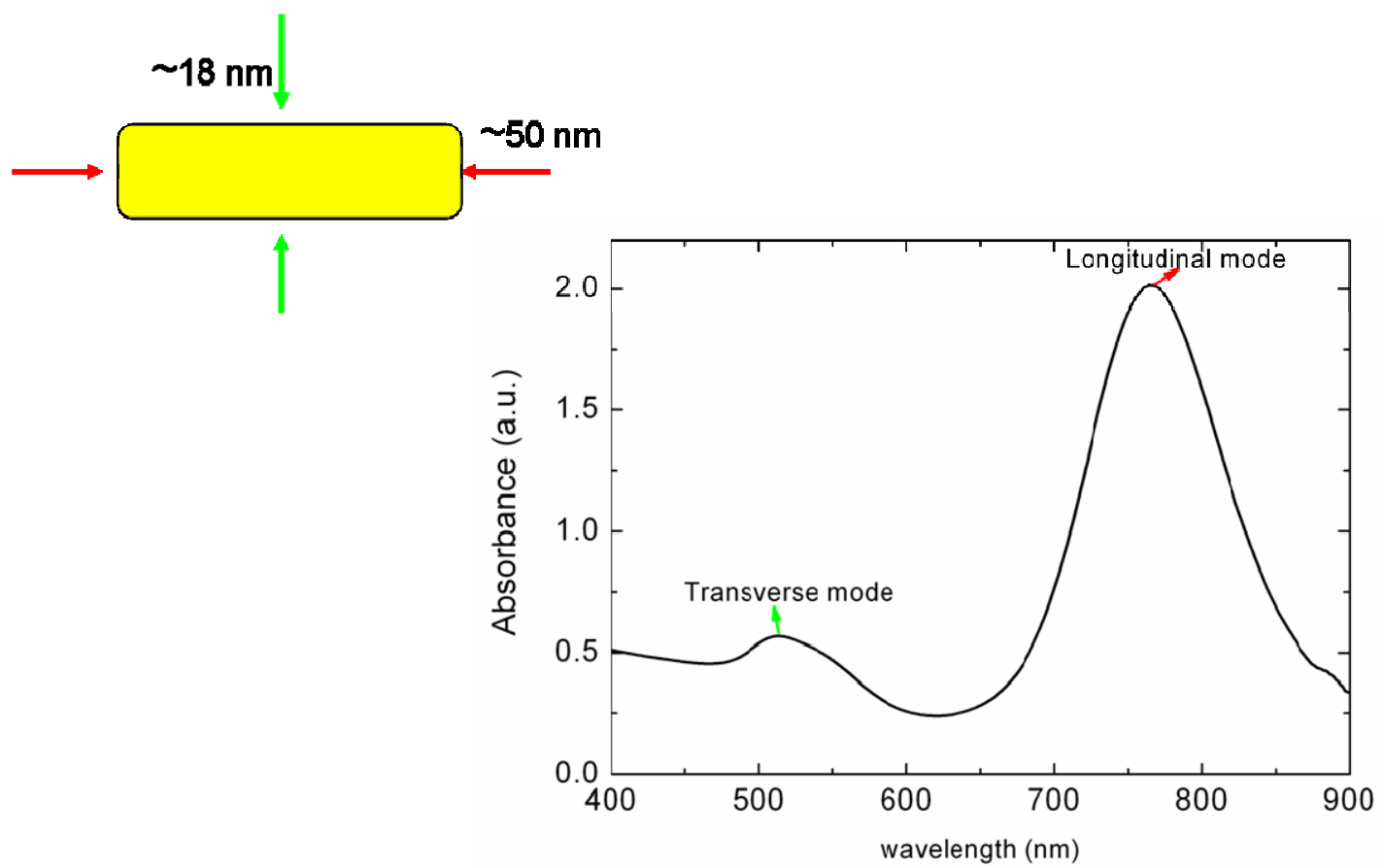

Figure 4: Schematic representation of axis of gold nanorods and optical spectrum of gold nanorods. The spectrum shows gold nanorods having a transverse plasmon peak representing the short axis in the vicinity of $\sim 516 \mathrm{~nm}$ and a longitudinal plasmon peak in the vicinity of $\sim 760 \mathrm{~nm}$. The longitudinal plasmon peak can be can be tuned from $600 \mathrm{~nm}$ to $850 \mathrm{~nm}$ with change in the aspect ratio (length/width) of nanorod.

Due to the tunability of the longitudinal plasmon peak of gold nanorods, they are suitable for deep in vivo imaging applications. Synthesis of gold nanorods with distinct and reproducible aspect ratios with a high yield is then a requirement.

\section{Synthesis of gold nanorods}

The commonly used method for synthesis of gold nanorods is a 3-step method, in which a citrate seed solution is used $[9,11]$. 
I. Synthesis of seed

$2.5 \times 10^{-4} \mathrm{M} \mathrm{HAuCl}_{4}+$

$2.5 \times 10^{-4} \mathrm{M} \mathrm{Na}$-citrate
Gold nanoparticle seeds

( 4nm diameter)
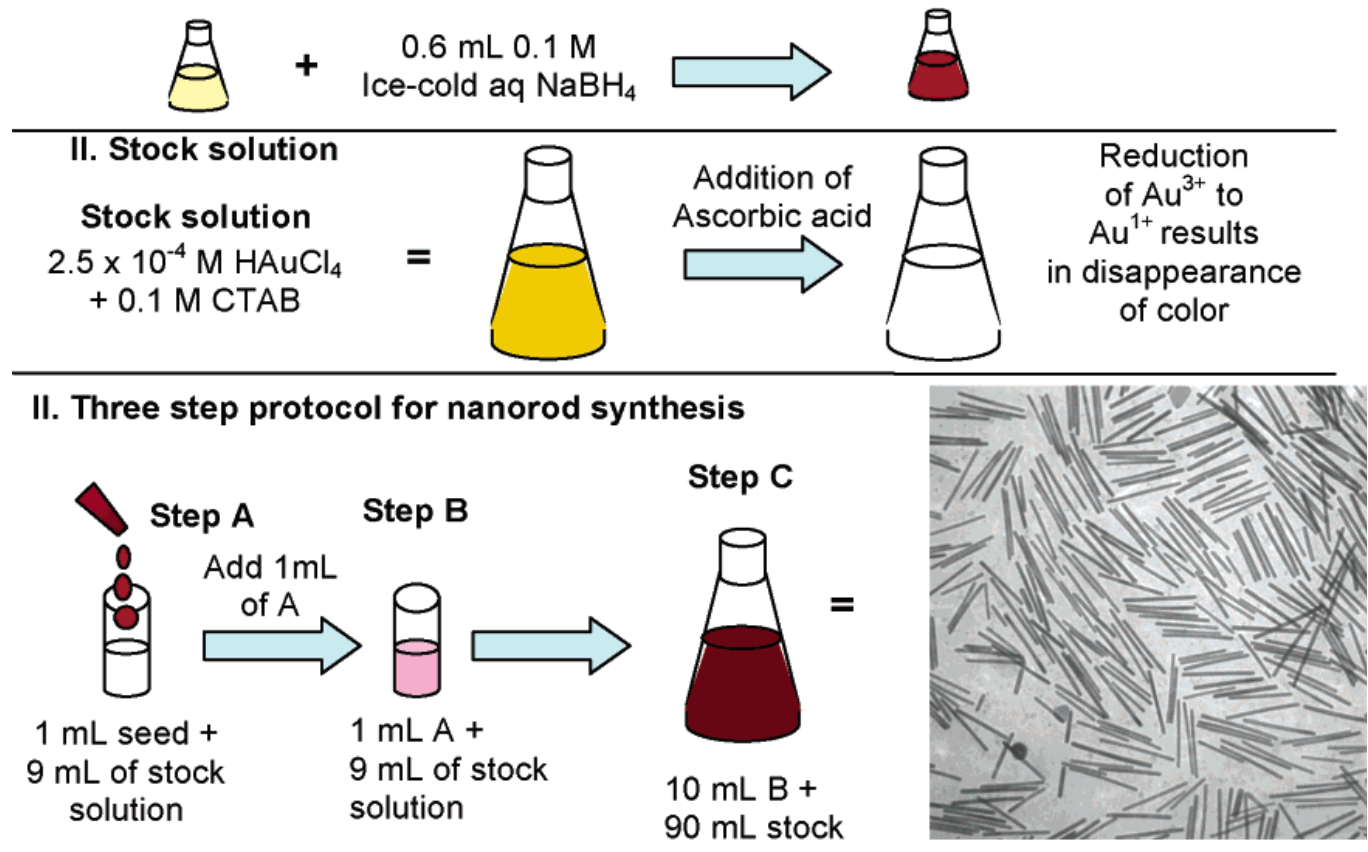

Figure 5: Seed-mediated growth approach to making gold and silver nanorods of controlled aspect ratio. The specific conditions shown here, for $20 \mathrm{ml}$ volume of seed solution, lead to high-aspect ratio gold nanorods. (bottom right) Transmission electron micrograph of gold nanorods that are an average of $500 \mathrm{~nm}$ long. (Reproduced with permission from Ref. 9)

In this method as depicted in figure 5, citrate capped seed are used as nucleating structures. This seed solution is used further in a 3-step method involving growth solutions (CTAB and gold salt). The growth solution is further supplemented with ascorbic acid that converts $\mathrm{Au}^{3+}$ to $\mathrm{Au}^{1+}$ oxidation state changing the orange colored solutions to colorless. Further, addition of citrate seed solution to the solution yields gold nanorods.

To adjust the size, the mechanism of nanorod formation is important to understand. Murphy et al [9] proposed the following mechanism of nanorod formation using citrate capped seed particles as shown in figure 6. The face centered cubic (fcc) crystal of gold have certain facets such as $\{111\},\{100\}$ and $\{110\}$. The development of facets occurs from the nucleating citrate capped seed crystals. The surfactant (CTAB) preferentially binds to $\{100\}$ facet. The continuous deposition of $\mathrm{Au}$ and its reduction by ascorbic acid (weak reducing agent) lead to growth in 1-dimensional manner at the exposed facets $\{111\}$ in pentatetrahedral twin formation. The $\mathrm{CTAB}$ thus forms a bilayer on the less stable facets $\{100\}$ on the side-surfaces of the nanorod. 
STEP 1: SYMMETRY BREAKING IN FCC METALS

STEP 2: PREFERENTIAL SURFACTANT BINDING TO SPECIFIC CRYSTAL FACES

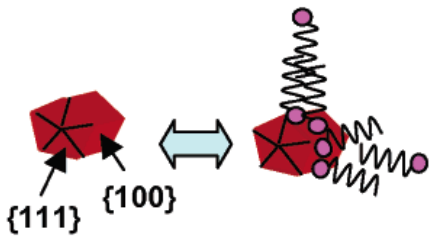

A) BINDING OF CTAB
TO AU(100) FACE

$$
\xi=\mathrm{CTAB}
$$

THE POSITIVELY CHARGED CTAB BILAYER STABILIZES THE NANORODS

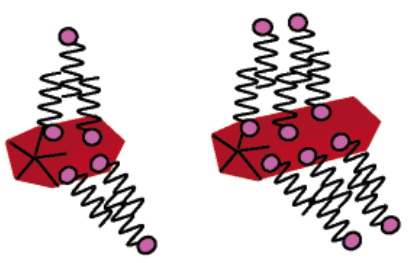

B) CONTINUED GROWTH IN 1-D UNTIL THE REAGENTS ARE EXHAUSTED
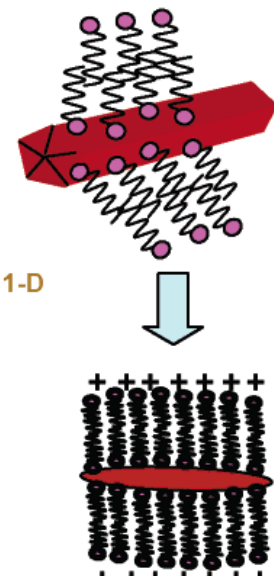

Figure 6: Proposed mechanism of surfactant-directed metal nanorod growth. The single crystalline seed particles have facets that are differentially blocked by surfactant (or an initial halide layer that then electrostatically attracts the cationic surfactant). Subsequent addition of metal ions and weak reducing agent lead to metallic growth at the exposed particle faces. In this example, the pentatetrahedral twin formation leads to $\mathrm{Au}$ $\{111\}$ faces that are on the ends of the nanorods, leaving less stable faces of gold as the side faces, which are bound by the surfactant bilayer. (Reproduced with permission from Ref. 9)

This synthesis procedure had drawbacks on final yield and additional by-products such as nanotriangles, nanoplates were formed with low yield of gold nanorods. The nanorods formed are long (up to $500 \mathrm{~nm}$ ) with a maximal absorbing wavelength of $\sim 1750 \mathrm{~nm}$ and, as they do not fall within the optical therapeutic imaging window/biological window (600 nm$950 \mathrm{~nm}$ ), are less suitable for biomedical imaging.

Nikoobakht and El-Sayed [37] proposed a synthesis procedure of gold nanorods using silverassisted surfactant method where pre-formed CTAB-stabilized Au seeds are used as nucleating particles. With the use of silver nitrate in the growth solutions, the procedure produces nanorods that absorb between $600 \mathrm{~nm}-850 \mathrm{~nm}$ in high yield and monodispersity. However, stability of nanoparticles is still a concern as CTAB stabilized gold nanorods aggregate at room temperature due to crystallization of CTAB present on the side-surfaces of nanorods $[9,11]$. As the excess $\mathrm{CTAB}$ present in the solution of gold nanorods is toxic to cells, the use of gold nanorods is limited for several biomedical applications in vivo. For making gold nanorods biocompatible, the surfaces should be treated with polymers that give stability to the nanoparticles [38]. 


\section{Stability of gold nanospheres and gold nanorods}

The stability of CTAB capped gold nanoparticles can be improved by coating the surfaces of nanoparticles with various polyelectrolytes or biodegradable polymers. Several studies have shown the use of polyethylene glycol (PEG) as a stable polymer to coat the surfaces to prevent them from clustering or aggregation. The PEG molecule is easily soluble in water, and can be terminated with functional molecules at its ends. PEGylation of nanoparticles also helps in providing a stealth character [38] when administered in the blood stream of living organisms that allows them to evade the immune response and so that they do not interact with cells of the reticulo-endothelial system (RES). These characteristics make PEG an essential polymer for providing stability and use in in vivo applications.

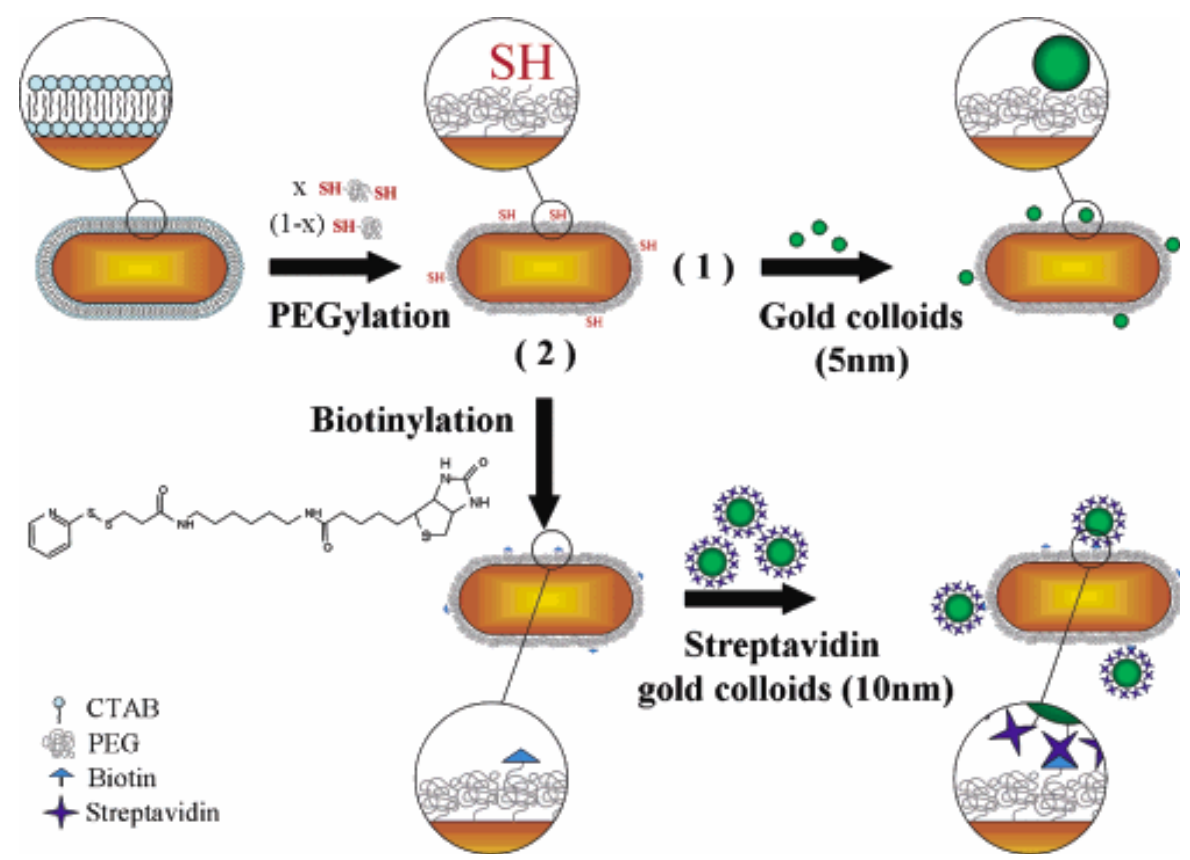

Figure 7: Schematic representation of the gold nanorod functionalization strategy: Mixed monofunctional and bifunctional PEG chains are first grafted on the surface of the rods (PEGylation). The free thiol groups are then reacted with either (1) small gold colloid ( $5 \mathrm{~nm}$ ), leading to rods decorated with small colloids, or (2) a biotin derivative. Gold colloids $(10 \mathrm{~nm})$ coated with streptavidin can be then immobilized on the surface of the nanorods (Reproduced with permission from Ref. 39).

Stabilization by polymers such as PEG-SH (thiolated PEG) as recently shown by Sonnischen et al [39] also can be used to self-assembly gold nanorods (see figure 7).

In this manner, the spherical and rod shaped gold nanoparticles are made stable and biocompatible with treatment with polymers and functionalization with ligands such as biotin/streptavidin [39] leads the way for imaging as well as therapeutic applications in the field of medicine and biology. 


\section{Aim/Scope of the thesis}

The aim of the thesis is to develop functionalized gold nanorods as molecular probes that are biocompatible, stable, with the ability to target tumor cells and consequently can be used in vivo for light-based imaging and treatment techniques (see figure 8).

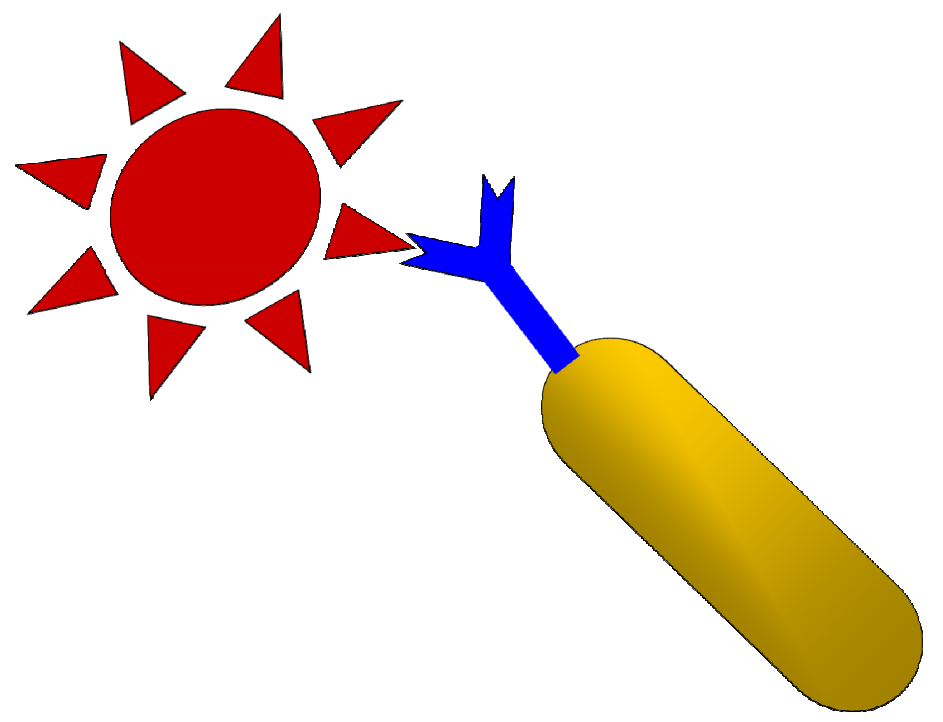

Figure 8: Contrast agent/Molecular probe where gold nanorod (represented in yellow) can be functionalized by attaching an antibody (represented in blue) that could bind to the receptors (represented in red) of tumor cell and hence can act as a molecular probe for biomedical imaging applications in therapy.

To reach that goal, the following research questions have to be answered:

1) How to synthesize and optimize size/shape of gold nanorods suitable for the NIR imaging window $(600 \mathrm{~nm}-950 \mathrm{~nm})$ in high yield and monodispersity?

2) What is the influence of several chemical components used in the synthesis procedures on nanoparticle shape, size and yield?

3) How to develop molecular probes by functionalizing gold nanorods with suitable antibodies that bind strongly to the receptors of tumor cells?

4) How to stabilize gold nanorods and increase their shelf-life and prevent aggregation?

5) What is the effect in vitro on different cell lines and in vivo biodistribution studies of gold nanorods?

To answer the above research questions, we first have opted to focus on synthesis procedures to improve the yield of gold nanorods by having control on the parameters during synthesis, 
as described in Chapter 2. In that chapter, we use the wet chemical synthesis of gold nanorods based on the seed-mediated silver assisted mono-surfactant method. We also describe the bioconjugation of gold nanorods to HER81 mAb (monoclonal antibody) via electrostatic/hydrophobic binding.

Chapter 3 shows that certain suppliers of CTAB having same product numbers do not synthesize nanorods. This is due to the presence of iodide impurities that can vary significantly from lot-to-lot within a product, to such an extent that there is no guarantee that gold nanorods can be synthesized with one or other CTAB product.

Chapter 4 shows that certain chemical components have influence on the shape/size and and therefore optical properties of nanoparticles. The increased addition of ascorbic acid volumes during synthesis causes efficient reduction of available gold ions that increases the sizes of the particles while changing their shape from nanorod to dog-bone, dumbbell and irregular shaped nanostructures.

Chapter 5 shows the toxicity studies in vitro on several cell lines. We found that the cell viability is heavily dependent on the type of coating (CTAB capped versus polymer coated gold nanorods).

Chapter 6 describes the in vivo biodistribution studies of gold nanorod in small animals after intravenous injection of CTAB capped and PEGylated gold nanorods. The accumulation of nanorods in different organs of rat is shown to be dependent on the type of coating present on the surface of nanoparticle. The coating also affects circulation times of nanoparticles in blood. These results recommend the type of coating on nanorods that are make them suitable and biocompatible for applications in vivo.

Chapter 7 describes the method of covalent bioconjugation of gold nanorods using PEGlinker (via thiol linkage). The toxicity and uptake of conjugated gold nanorods by breast carcinoma cell line are reported.

Chapter 8 discusses the conclusions of this thesis and recommendations/outlook for using gold nanorods as molecular probes for in vivo applications are presented. 


\section{References:}

1) M. Faraday, Philos. Trans. R. Soc. London, 147, pp. 145-181, 1857.

2) C.N. Rao, G.U. Kulkarni, P.J. Thomas and P. Edwards, Chem. Soc. Rev., 29, pp. 27-35, 2000.

3) C.B. Murray, D.J. Noms and M.G. Bawendi, J. Am. Chem. Soc., 115, 8706, 1993.

4) S.H. Yu, M. Yoshimura, J.M.C. Moreno, T. Fujiwara, T. Fujino, R. Teranishiet al., Langmuir, 17, 1700, 2001.

5) S. Eustis and El-Sayed, M. A., Chem. Soc. Rev. 35, 209, 2006.

6) H.H. Richardson, Z.N. Hickman, A.O. Govorov, A.C. Thomas, W. Zhang, M.E. Kordesch, Nano Lett., 6, pp. 783-788, 2006.

7) A.O. Govorov and H.H. Richardson, 2, Nano Today, 2007.

8) Y. Yu, S. Chang, C. Lee and C.R. Chris Wang, J. Phys. Chem. B, 101, pp. 6661-6664, 1997.

9) C.J. Murphy, T.K. Sau, A.M. Gole, C.J. Orendorff, J. Gao, L. Gou, S.E. Hunyadi and T. Li, J. Phys. Chem. B, 109, pp. 13857-13870, 2005.

10) N.R. Jana, L. Gearheart and C.J. Murphy, Adv. Mater, 13, pp. 1389-1393, 2001.

11) N.R. Jana, L. Gearheart, and C.J. Murphy, J. Phys. Chem. B., 105, pp. 4065-4067, 2001.

12) J. Perez-Juste, I. Pastoriza-Santos, L. Liz-Marzan and P. Mulvaney,., Coordination Chemistry Reviews., 249, pp. 1870-1901, 2005.

13) W. Cai, T. Gao, H. Hong and J. Sun, Nanotechnology, Science and Applications, 1, pp. 17-32, 2008.

14) G.F. Paciotti, L. Myer, D. Weinreich, D. Goia, N. Pavel, R.E. McLaughlin and L. Tamarkin, Drug Deliv., 11, pp. 69-183, 2004.

15) P. Mukherjee, R. Bhattacharya, N. Bone, Y.K. Lee, C.R. Patra, S. Wang, L. Lu, S. Charla, P.C. Banerjee, M.J. Yaszemski, J. Nanobiotechnology, 5, 2007.

16) I. El-Sayed, X. Huang, F. Macheret, J.O. Humstoe, R. Kramer, M.A. El-Sayed, Technol Cancer Res Treat, 6, pp. 403-412, 2007.

17) J. Turkevich and J. Hillier, Anal. Chem., 21, 475, 1949.

18) J. Turkevich, P.C. Stevenson and J. Hillier, Discuss. Faraday Soc.

19) T. Yonezawa, K. Yasui and N. Kimizuka, Langmuir, 17, 271, 2001.

20) T. Teranishi, I. Kiyokawa, M. Miyake, Adv. Mater., 10, 596, 1998.

21) S.H. Chen and K. Kimura, Langmuir, 15, 1075, 1999.

22) M.Q. Zhao, L. Sun and R.M. Crooks, J. Am. Chem. Soc., 120, 4877, 1998.

23) P.K. Jain, I.H. El-Sayed and M.A. El-Sayed, Nano Today, 2, pp. 18-29, 2007.

24) L. Brannon-Peppas and J.O. Blanchette, Adv. Drug Deliver. Rev. 56, pp. 1649-1659, 2004.

25) D.F. Baban and L.W. Seymour, Adv. Drug Deliv. Rev. 34, pp. 109-119, 1998.

26) B.D. Chithrani, A.A. Ghazani, W.C. Chan, Nano Lett., 6, pp. 662-668, 2006.

27) M. Thomas and A.M. Klibanov, Appl. Microbiol. Biotechnol. 62, pp. 27-34, 2003.

28) C.M. McIntosh, E.A. Esposito, A.K. Boal, J.M. Simard, C.T. Martin and V.M. Rotello, J. Am. Chem. Soc. 123, pp. 7626-7629, 2001.

29) H. Wang, Y. Chen, X.Y. Li and Y. Liu, Mol. Pharm. 4, pp. 189-198, 2007.

30) A. Verma, J.M. Simard, J.W.E. Worrall and V.M. Rotello, J. Am. Chem. Soc. 126, pp. 13987-13991, 2004.

31) D.R. Bhumkar, H.M. Joshi, M. Sastry and V.B. Pokharkar, Pharm. Res. 24, pp. 1415-1426, 2007.

32) P.K. Jain, K.S. Lee, I.H. El-Sayed and M.A. El-Sayed, J. Phys. Chem. B, 110, 2006.

33) A.S. Angelatos, B. Radt and F. Caruso, J. Phys. Chem., B., 109, pp. 3071-3076, 2005.

34) A.G. Skirtach, A.M. Javier, O. Kreft, K. Kohler, A.P. Alberola, H. Mohwald, W.J. Parak and G.B. Sukhorukov, Angew. Chem. Int. Ed. 45, pp. 4612-4617, 2006.

35) R. Weissleder, Nature Biotechnology, 19, pp. 316-317, 2001.

36) P.K. Jain, X. Huang, I.H. El-Sayed, M.A. El-Sayed, Acc Chem Res., 1, pp. 1578-1586, 2008.

37) B. Nikoobakht and M.A. El-Sayed, Chem. Mater, 15, pp. 1957-1962, 2003.

38) T. Niidome, M. Yamagata, Y. Okamoto, Y. Akiyama, H. Takahashi, T. Kawano, Y. Katayama, Y. Niidome. J Cont. Release, 12, pp. 343-347, 2006.

39) S. Pierrat, I. Zins, A. Breivogel, and C. Sönnichsen, NanoLett., 7, pp. 259-263, 2007. 
Chapter 2

\title{
Synthesis and bioconjugation of gold nanoparticles as potential molecular probes for light-based imaging techniques
}

\begin{abstract}
We have synthesized and characterized gold nanoparticles (spheres and rods) with optical extinction bands within the "optical imaging window". The intense plasmon resonant driven absorption and scattering peaks of these nanoparticles make them suitable as contrast agents for optical imaging techniques. Further, we have conjugated these gold nanoparticles with an anti-HER-2-neu mouse monoclonal antibody $(\mathrm{mAb})$ that is specific to the SKBR3 breast carcinoma cell line. The bioconjugation protocol uses non-covalent modes of binding based on a combination of electrostatic and hydrophobic interactions of the antibody and the gold surface. We discuss various aspects of the synthesis and bioconjugation protocols and the characterization results of the functionalized nanoparticles. Some proposed applications of these potential molecular probes in the field of biomedical imaging are also discussed.
\end{abstract}

This chapter published as "R.G. Rayavarapu, W. Petersen, C. Ungureanu, J. Post, T.G. van Leeuwen and S. Manohar", International Journal of Biomedical Imaging, 29817, 2007. 


\section{Introduction}

Optical imaging encompasses a multitude of techniques for the elucidation of morphology, molecular function and metabolism of tissue with the general objective of detecting, diagnosing, staging and treatment monitoring of disease. Progression of disease is usually accompanied by changes in physiology and pathology that are manifested as location specific changes in optical properties thereby affording contrast for optical imaging to study disease.

Optical imaging techniques span the range from surface to bulk imaging systems with applications ranging from "optical biopsies" to full human breast imaging with resolutions that cover the microscopic to macroscopic. Some important imaging techniques for superficial tissue imaging are confocal microscopy [1], two-photon microscopy [2] and optical coherence tomography (OCT) [3]. Techniques that permit sub-surface to deep imaging are diffuse optical imaging (DOT) [4] and photoacoustic imaging [5].

The interaction of visible and nearinfrared (NIR) light with tissue is dominated by:

a) absorption processes which are due to the presence of various chromophores such as hemoglobin, oxy-hemoglobin, melanin, water and lipids [6].

b) scattering processes due to the cell membrane and cell structures such as the nucleus, mitochondria, lysosomes etc [6].

Penetration of light in tissue is dependent on the extent of the two processes above and is low in the high-energy visible region of the spectrum. This is due to high absorption by hemoglobin and severe light scattering. In the wavelength regime between $600 \mathrm{~nm}$ and 1300 $\mathrm{nm}$, absorption and scattering losses are minimal permitting high light penetration. This is the so-called "optical imaging window" which is exploited for deep imaging in tissue [7].

The sensitivity and specificity of optical imaging techniques is governed by contrast: the ability of the disease to differentially scatter or absorb light compared with non-pathological tissue and background noise. This native or endogenous contrast may not be sufficient and in any case, the interactions of light with tissue are not disease- specific. There is thus a role for exogenously administered contrast enhancing agents that have affinity for the disease site through biochemical interactions, providing not only sensitive but also disease-specific signals.

Contrast agents for optical imaging thus far have been near-infrared dyes based on cyanine dyes [8] such as Indocyanine Green [9], but in the last few years, gold nanoparticles [10, 11, 
12] have shown themselves to be prime candidates due to their unusual optical properties and inherent biocompatibility.

Gold metal nanoparticles (NPs) exhibit narrow and intense absorption and scattering bands due to the phenomenon of plasmon resonance. This occurs at the resonance condition of the collective oscillation that conduction electrons experience in an electromagnetic field of the appropriate wavelength [13]. The plasmon resonant condition of gold NPs depends upon their size, shape, structure (solid or hollow), and aggregation on the embedding medium. Spherical gold nanoparticles have a single plasmon resonant extinction peak at around $520 \mathrm{~nm}$, which does not shift extensively with changes in size and refractive-index of the surrounding medium. This is a wavelength at which light penetration in tissue is poor due to strong scattering and absorption by hemoglobin, and gold nanospheres are not ideal for deep imaging in tissue.

Rod shaped NPs exhibit two plasmon resonances due to oscillation of the conduction electrons along the short axis and along the long axis. The former plasmon band is called the transverse resonance and the latter the longitudinal resonance. While the transverse plasmon band occurs in the neighborhood of $520 \mathrm{~nm}$, the longitudinal band is red-shifted. The extent of the red-shift depends on the aspect ratio of the gold nanorod (AuNR); the higher the aspect ratio, the further the shift. Thus by tailoring the length and/or width of these particles, their extinction peaks may be made theoretically to cover the low-energy visible to infrared wavelength regions.

The intense scattering and absorption of light that occurs under the plasmon resonant condition coupled with the ability to tune the resonance into the near-infra red (NIR) by manipulating the aspect ratio, makes gold nanorods extremely attractive as contrast agents for optical imaging techniques. Further, gold-protein chemistry is well developed and several bioconjugation protocols are available in the literature, which could allow the combination of the targeting functionality of antibodies with such gold NPs. The inertness and biocompatibility of gold in general, holds promise the use of gold NPs for in vivo imaging applications.

Gold NPs can be synthesized using wet chemical methods, which are based on the reduction of gold salts by reducing agents such as sodium borohydride and ascorbic acid. Seed mediated methods dominate wet chemical synthesis routes. These involve the reduction of gold using weak reducing agents onto small nanospheres of gold as seed; in the presence of 
shape directing surfactants usually cetyltrimethylammonium bromide (CTAB). These methods may be distinguished into those that use silver ion assistance in growth solutions and those that do not.

Murphy and co-workers described the three-step growth protocol $[14,15]$, where medium to high aspect ratio nanorods could be synthesized, without the use of silver nitrate. Seed particles are generated by reducing gold salt using sodium borohydride in the presence of sodium citrate. The spheres are coated with a layer of negatively charged citrate ions that maintain colloid stability against aggregation by electrostatic repulsion. These spheres seed a growth solution comprising gold salt, CTAB and ascorbic acid in three steps thereby slowing down reduction. The mechanism of nanorod formation by this method is not yet fully understood. Murphy and co-workers [3] proposed that the polar CTA ${ }^{+}$head group of the surfactant binds with greater preference to certain crystallographic faces thereby passivating them to the deposition of gold. The other faces on the other hand, would be exposed for gold to be reduced on, thereby producing anisotropic growth into rods.

The methods using silver nitrate in the growth solutions, were proposed by Jana et al. [16], but modified by Nikoobakht and El-Sayed [17] to achieve spectacular yields of nanorods with excellent monodispersity. Importantly, they also showed that changing the quantity of $\mathrm{Ag}^{+}$ions in the growth solution allows for fine-tuning of the aspect ratios of the nanorods. The mechanism at work in this protocol has been debated in the recent past. One mechanism postulates $\mathrm{CTAB}$ as a soft template that elongates on addition of $\mathrm{Ag}^{+}$ions, which occupy regions between the $\mathrm{CTA}^{+}$head groups to reduce the repulsion between the head groups [17]. A second mechanism invokes the CTAB passivation concept with additional adsorption of silver bromide on facets slowing down reduction and producing rods shorter than those made without using $\mathrm{Ag}^{+}$[18]. A third mechanism which has appeared recently [19], proposes the underpotential deposition (UPD) of $\mathrm{Ag}^{0}$ on certain facets, followed by CTAB binding, which serves to stabilize the faces, and allowing gold reduction on other faces resulting in rod formation.

In this article, we present our experiences in synthesizing gold nanospheres and nanorods using slight modifications to the protocols discussed above. Our goal is to obtain nanorods whose aspect ratios can be tuned to obtain plasmon peaks between $650 \mathrm{~nm}-850 \mathrm{~nm}$. Next, we conjugate the gold nanospheres and gold nanorods to the HER81 monoclonal antibody using electrostatic and hydrophobic interactions. The conjugation does not use modifications of the 
bilayer charge of nanorods nor does it uses any linkers. We discuss various aspects of these protocols and postulate a possible mechanism for the bioconjugation of the antibody with the gold nanorods. We also discuss the feasibility of using these molecular probes for contrast enhancement of photoacoustic cancer imaging using simulations.

\section{Materials And Methods}

\section{Gold nanorods using the silver assisted single surfactant growth method}

The following are the reagents used for the synthesis of the gold seed and gold nanorods: Tetrachloroauric acid (Acros Organics), cetyltrimethylammonium bromide CTAB $>99 \%$ (Fluka), sodium citrate (Sigma), $\mathrm{NaBH}_{4}$, sodium borohydride 99\% (Aldrich), ascorbic acid 99\% (Aldrich), $\mathrm{AgNO}_{3}$, silver nitrate 99.8\% (Merck). All reagents were used as received. Prior to use, all glassware was cleaned with $\mathrm{HF}$, further with aquaregia $\left(\mathrm{HCl} / \mathrm{HNO}_{3}\right)$ and rinsed twice with Milli Q water.

As mentioned earlier the seed-mediated protocol requires the use of small gold nanospheres to seed growth solutions with silver nitrate as per the protocol of Nikoobakht and El-Sayed [17].

\section{Gold seed of $3.5 \mathrm{~nm}$ diameter}

Five $\mathrm{ml}$ of $0.2 \mathrm{M} \mathrm{CTAB}$ is sonicated for 20 minutes at $40^{\circ} \mathrm{C}$ in a water bath. To $5 \mathrm{ml}$ of this in an inert atmosphere with nitrogen gas, $5 \mathrm{ml}$ of $\mathrm{HAuCl}_{4} .3 \mathrm{H}_{2} \mathrm{O}(0.0005 \mathrm{M})$ is added with continuous stirring. Then $0.6 \mathrm{ml}$ of ice-cold $0.01 \mathrm{M} \mathrm{NaBH}_{4}$ is added all at once with vigorous stirring for 1 minute. The color of the CTAB-capped seed solution is light brownish yellow.

\section{Gold nanorods of varying aspect ratios}

The growth solution consists of CTAB $(0.2 \mathrm{M})$ and $\mathrm{HAuCl}_{4} .3 \mathrm{H}_{2} \mathrm{O}(0.0005 \mathrm{M})$. The color is dark yellow. Five such identical solutions of $5 \mathrm{ml}$ were prepared. Appropriate volumes of $\mathrm{AgNO}_{3}(0.006 \mathrm{M})$ depending on the desired aspect ratio of nanorods were added to each growth solution. The mild reducing agent ascorbic acid $(0.078 \mathrm{M})$ was added to each solution that turns the solutions colorless. Finally, $14 \mu 1$ of 8 minutes aged, preformed CTAB-capped seed solution was added to each conical flask and gently mixed. It was allowed to stand for 3 hours at a temperature of $24^{\circ} \mathrm{C}$. The color of the nanorod suspensions are dark blue with a brownish opalescence. These sols were then concentrated by centrifuging which also removes the excess unbound $\mathrm{CTAB}$, and then stored at $4^{\circ} \mathrm{C}$. 


\section{Characterization of gold nanoparticles}

Electron microscopy of the NPs was performed using a CM 30 Philips Transmission Electron Microscope (TEM) or a Zeiss-1550 high-resolution Scanning Electron Microscope (HRSEM). Particle sizes were estimated using NI Vision Module (LABVIEW) on the digital SEM images with at least 250 particles considered in each case. Extinction spectra of NPs (and bioconjugated NPs) were measured using the Shimadzu PC3101- UV-VIS-NIR spectrophotometer.

The concentration of nanorods synthesized was estimated using the relation $A=c d \varepsilon$, where $A$ is the measured absorbance, $c$ the concentration in $\mathrm{M}, \varepsilon$ the molar extinction coefficient $\left(\mathrm{M}^{-}\right.$ ${ }^{1} \mathrm{~cm}^{-1}$ ) and $d$ the path length of the cuvette used to record the spectra. The molar extinction coefficients can be obtained from a recent report where $\varepsilon$ values were estimated for a range of aspect ratios of nanorods by measuring the gold content in sols using inductively coupled plasma (ICP) atomic emission spectroscopy [19].

\section{Bioconjugation of HER81 mAb to gold nanoparticles}

Conjugation was achieved using combination of electrostatic and hydrophobic binding interactions. The particles chosen for bioconjugation were $25 \mathrm{~nm}$ citrate-capped gold spheres (Aurion, Wageningen, The Netherlands), and silver-assisted surfactant mediated gold nanorods with aspect ratios approximately 2.85 (See Table 1) with the longitudinal plasmon peak at $764 \mathrm{~nm}$. The anti-HER-2 monoclonal antibody was chosen as the targeting moiety. The antibody designated as HER81, is a mouse mAb and recognizes an epitope on HER-2. HER-2 is a member of the epidermal growth factor receptor (EGFR) family and is over expressed in 20-40\% of human breast cancers [20].

In general, for optimum conjugation, it is recommended that the $\mathrm{pH}$ of the antibody and gold sol be maintained at or slightly higher than the isoelectric point ( $\mathrm{pI}$ ) of the antibody [21]. The isoelectric (pI) point of HER81 mAb was determined using the Pharmacia PhastSystem Iso Electric Focussing (IEF). The $\mathrm{pH}$ of the antibody was adjusted with dialysis in $5 \mathrm{mM}$ sodium acetate buffer and the $\mathrm{pH}$ of the colloidal gold was adjusted with $0.1 \mathrm{M} \mathrm{KOH}$, to approximately $0.5 \mathrm{pH}$ units above this value. 
Next, the minimum protecting amount of antibody to be used for the conjugation is determined. This is the amount of protein that is required to maintain colloidal stability of the conjugated NPs upon addition of $\mathrm{NaCl}$ [21] as judged by colorimetric analysis; as long as the conjugated NPs turns blue, particle aggregation takes place implying that the amount of protein is not sufficient to stabilize the suspension. By trial, different amounts of antibody are added to samples of the gold sol, gently mixed and allowed to stand at room temperature for 2 minutes. Spectroscopic analysis reveals which sample remains stable; the minimum amount of protein added is then ascertained and is used for subsequent conjugation of the gold sol.

To block the free surfaces on the gold, 10\% Bovine Serum Albumin (BSA) maintained at the same $\mathrm{pH}$ as antibody, is used. The BSA was added to the conjugate until the final concentration of $1 \% \mathrm{BSA}$ and allowed to incubate for 5 minutes. The resultant was then centrifuged for 30 minutes at $12000 \mathrm{~g}$ to remove excess of protein and incompletely stabilized particles. The resulting pellet is re-dispersed in phosphate buffered saline (PBS) in 1\% BSA and stored at $4^{\circ} \mathrm{C}$.

\section{Cell culture and cell-bioconjugate incubation}

HER81- positive mammary adenocarcinoma (SKBR3) was used as the positive cell line; Chinese Hamster Ovary (CHO) as the negative cell line. The cells were cultured in RPMI 1640 medium (Invitrogen) supplemented with glutamine, $10 \%$ FBS (Fetal Bovine Serum) with antibiotics. Cells were maintained in an incubator at $37{ }^{\circ} \mathrm{C}$ and $5 \% \mathrm{CO}_{2}$.

The cells were cleaved using trypsin, re-plated onto $12 \mathrm{~mm}$ glass cover slips in a 6-well tissue culture plate, and allowed to grow for 2 days in an incubator. When the cells grew to $80 \%$ confluence on the cover slips, the cells were rinsed with Phosphate Buffered Saline (PBS) and fixated in 4\% paraformaldehyde (PFA).

\section{Immunostaining and confocal microscopy}

After fixation, immunostaining was performed on the cells. The cells were incubated for 2 hours with the conjugated NPs and this was followed by silver enhancement performed using a silver-staining kit (Aurion, Wageningen, The Netherlands).

Confocal microscopy reflection images of the cells on cover slips were recorded on a Zeiss LSM 510 confocal laser-scanning microscope using a C-Apochromat 63 X/1.4 numerical aperture (NA) water-immersion objective. An excitation wavelength of $543 \mathrm{~nm}$ was chosen 
and reflection images recorded using a $500 \mathrm{~nm}-550 \mathrm{~nm}$ bandpass filter. All images were acquired with pinhole diameters of $178 \mu \mathrm{m}$. Care were taken to ensure that the excitation intensity as well as detector and amplifier gains were maintained at the same values for all images to facilitate comparison.

\section{Results}

\section{Synthesis of gold nanorods}

We used seed particles within about 5-8 minutes of formation in the subsequent growth phase. The optical extinction spectrum of aged seed is shown in Figure 1.

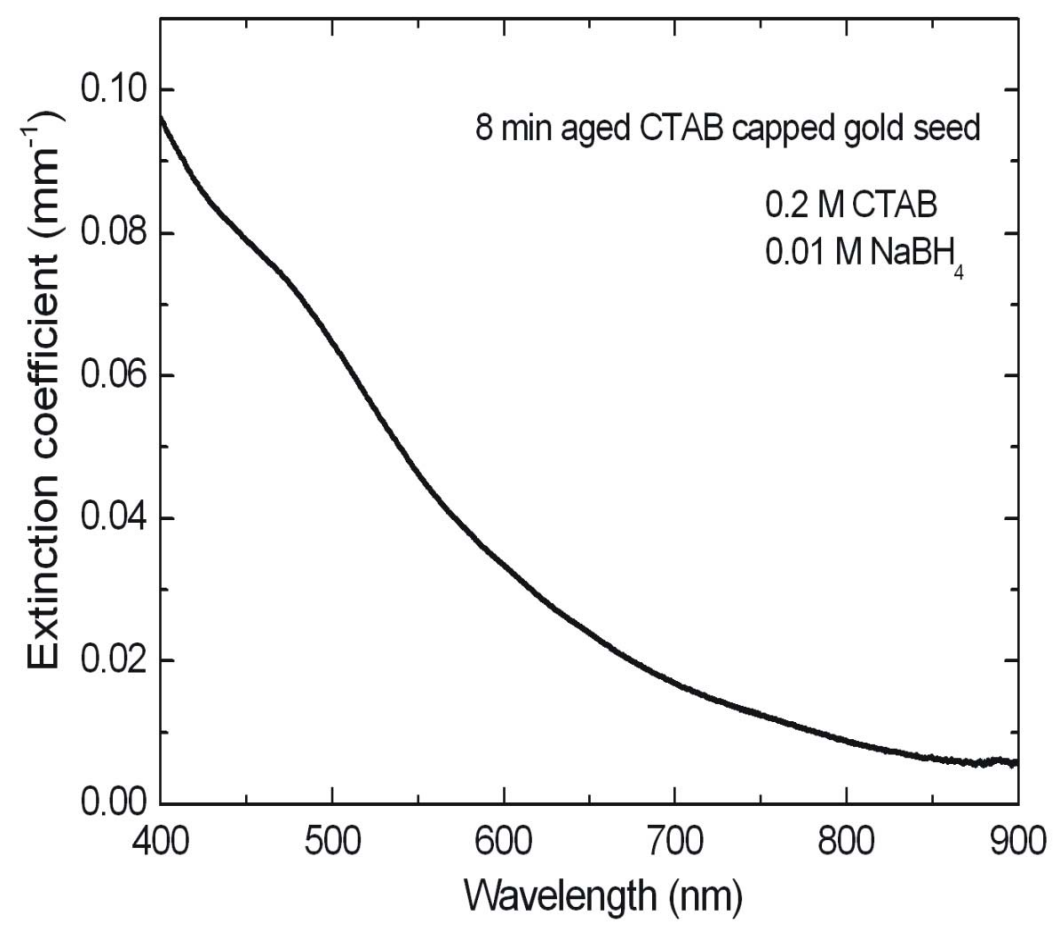

Figure 1: Optical extinction spectrum of preformed 8-minute-aged CTAB-capped gold nanospheres as seed for nanorod synthesis.

Figure 2 shows the extinction spectrum and SEM image of the nanorods synthesized using 50 $\mu 1 \mathrm{AgNO}_{3}$ in the growth solution. The peak at $675 \mathrm{~nm}$ can be attributed to longitudinal plasmon resonance and the peak in the vicinity of $516.5 \mathrm{~nm}$ to transverse plasmon resonance. 


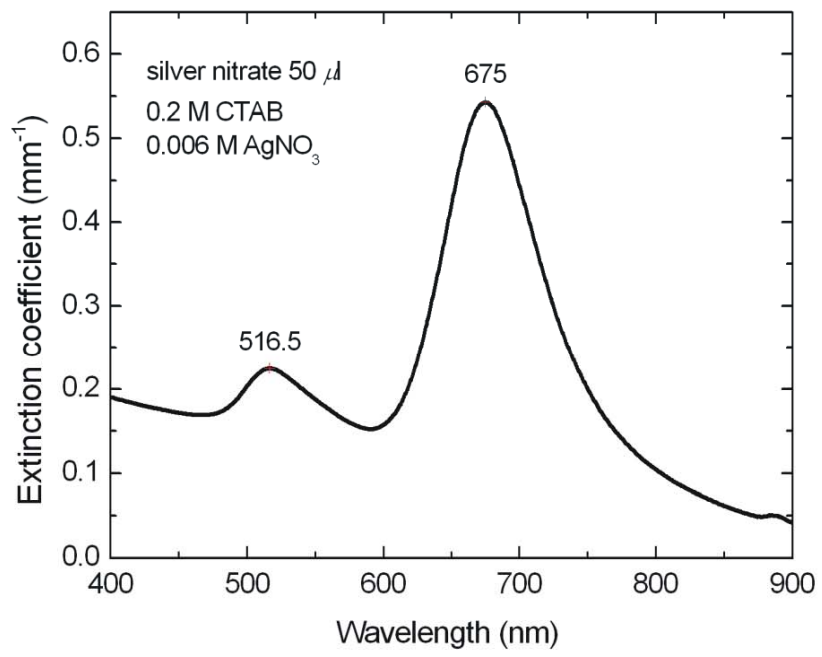

(a)

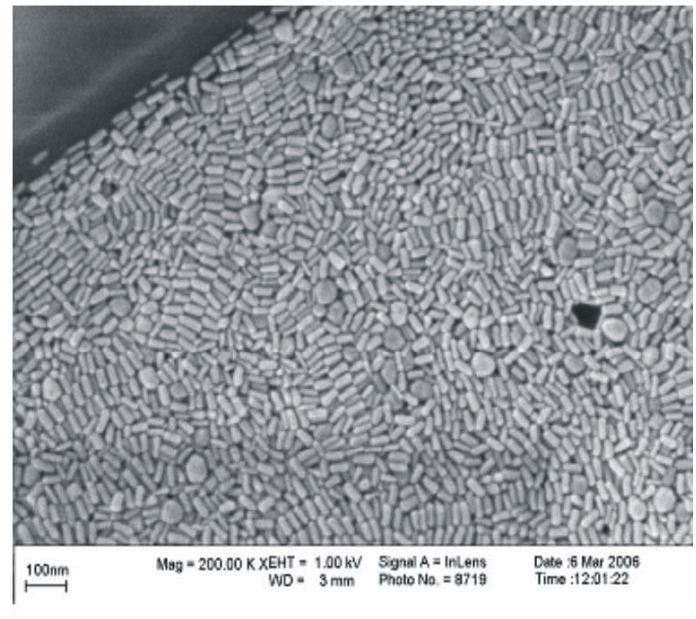

(b)

Figure 2: Gold nanorods synthesized using $50 \mu \mathrm{L}$ of silver nitrate in growth solution. (a) Optical extinction spectrum showing the transverse plasmon peak at $516.5 \mathrm{~nm}$ and the longitudinal plasmon peak at $675 \mathrm{~nm}$. The amplitude of the longitudinal plasmon peak is higher than transverse plasmon peak which indicates the formation of high yield of nanorods compared to spheres. (b) Scanning electron microscope (SEM) image of gold nanorods showing high monodispersity. Few nanospheres are observed.

Examination of the SEM image and determination of the mean sizes confirms this: the NPs produced consist of monodisperse nanorods of aspect ratio of $2.3 \pm 0.3$, with a small number of large spheres; the latter's extinction peak coinciding with the transverse plasmon band of the nanorods.

Figure 3 shows the extinction spectrum and the SEM image for the sample produced using $250 \mu l$ of silver nitrate. It is seen that the longitudinal plasmon band is shifted to $850 \mathrm{~nm}$.

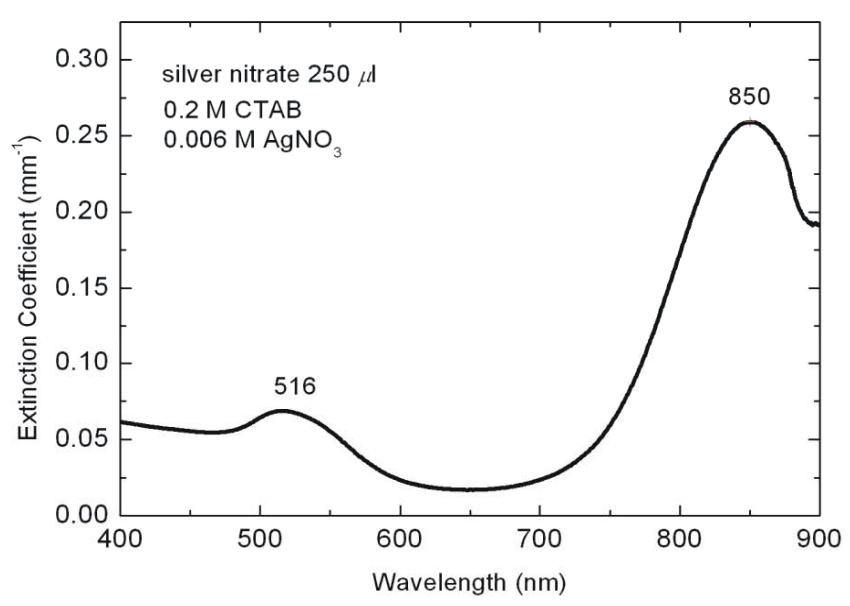

(a)

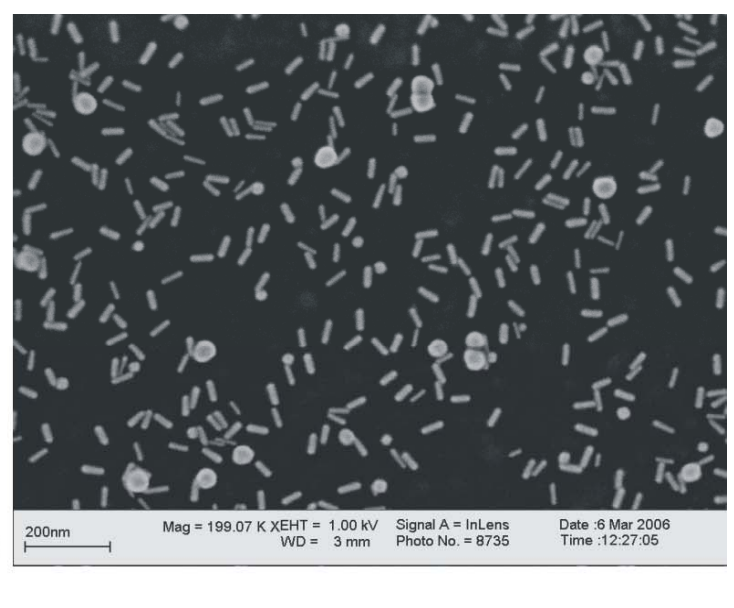

(b)

Figure 3: Gold nanorods synthesized using $250 \mu \mathrm{L}$ of silver nitrate in growth solution. (a) Optical extinction spectrum showing the transverse plasmon peak at $516 \mathrm{~nm}$ and the longitudinal plasmon peak at $850 \mathrm{~nm}$. (b) Scanning electron microscope (SEM) image. 
Sizing from the SEM image yields an average aspect ratio of $3.6 \pm 0.6$. Figure 6 shows the size statistics of the 2 specimens;

The values of the molar extinction coefficient for the 2 cases above are $(3.3 \pm 0.3) \times 10^{9}$ and (5.5 \pm 0.3$) \times 10^{9} \mathrm{M}^{-1} \mathrm{~cm}^{-1}$ obtained by extrapolation of the data from Ref. 19. With this, we arrive at the concentration of the nanorods with peak at $675 \mathrm{~nm}$ as $4.3 \pm 0.3 \times 10^{11} \mathrm{NR} / \mathrm{ml}$; for nanorods with the peak at $850 \mathrm{~nm}$ as $1.3 \pm 0.7 \times 10^{11} \mathrm{NR} / \mathrm{ml}$.

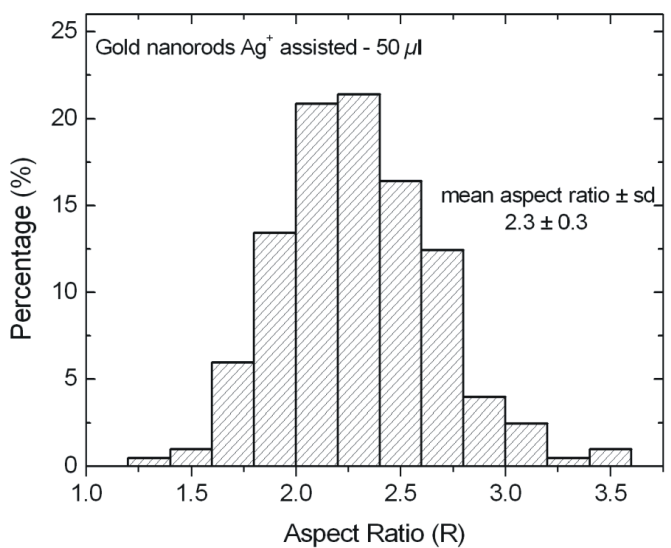

(a)

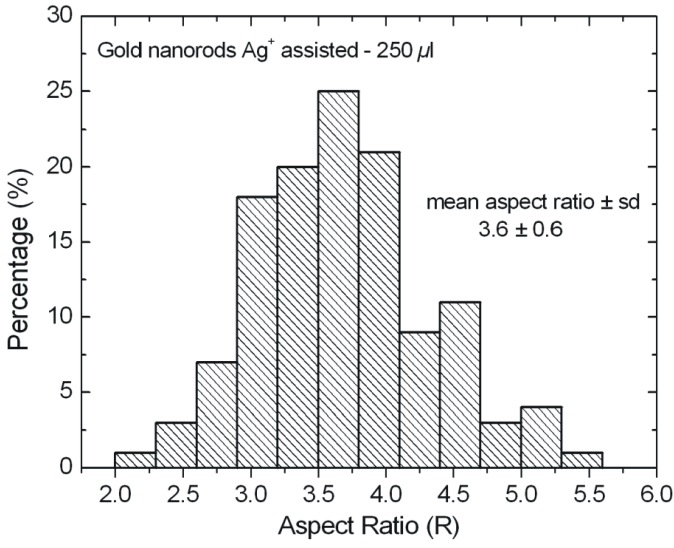

(b)

Figure 4: Histograms of gold nanorod aspect ratios synthesized with (a) $50 \mu \mathrm{L}$ silver nitrate, mean aspect ratio of $2.3 \pm 0.3$ (mean length $44.8 \pm 4.1 \mathrm{~nm}$, mean width $19.8 \pm 2.9 \mathrm{~nm}$ ); and with (b) $250 \mu \mathrm{L}$ silver nitrate, mean aspect ratio of $3.6 \pm 0.6$ (mean length $51.0 \pm 4.4 \mathrm{~nm}$, mean width $14.1 \pm 2.1$ ).

Figure 5 shows the consolidated normalized extinction spectra of 5 nanorod solutions, having identical growth solutions with varying silver nitrate volumes. The spectra were normalized to the peak at $516 \mathrm{~nm}$, which is due to a combination of the transverse plasmon resonance of the nanorods and the signature peak of gold nanospheres. It is seen that with higher silver nitrate volumes, the extent of red-shifting increases [22]. The details of the observed changes in aspect ratios and plasmon bands are presented in Table-1.

\begin{tabular}{cccccc}
\hline Sample & Volume of $\mathrm{AgNO}_{3}(\mu \mathrm{L})$ & Aspect ratio $(\mathrm{R})$ & Length $(\mathrm{nm})$ & Width $(\mathrm{nm})$ & LP band $(\mathrm{nm})$ \\
\hline 1 & 50 & $2.3 \pm 0.3$ & $44.8 \pm 4.1$ & $19.8 \pm 2.9$ & 675 \\
2 & 100 & $2.85 \pm 0.6$ & $45.1 \pm 5.5$ & $15.8 \pm 3.1$ & 764 \\
3 & 150 & $3.0 \pm 0.6$ & $41.7 \pm 3.9$ & $13.9 \pm 2.3$ & 788 \\
4 & 200 & $3.1 \pm 0.5$ & $52.0 \pm 4.6$ & $16.8 \pm 2.8$ & 831 \\
5 & 250 & $3.6 \pm 0.6$ & $51.0 \pm 4.4$ & $14.1 \pm 2.1$ & 850 \\
\hline
\end{tabular}

Table 1: Mean aspect ratios, lengths, widths, and longitudinal plasmon peaks for nanorods synthesized using the silver-assisted seed-mediated growth method. 


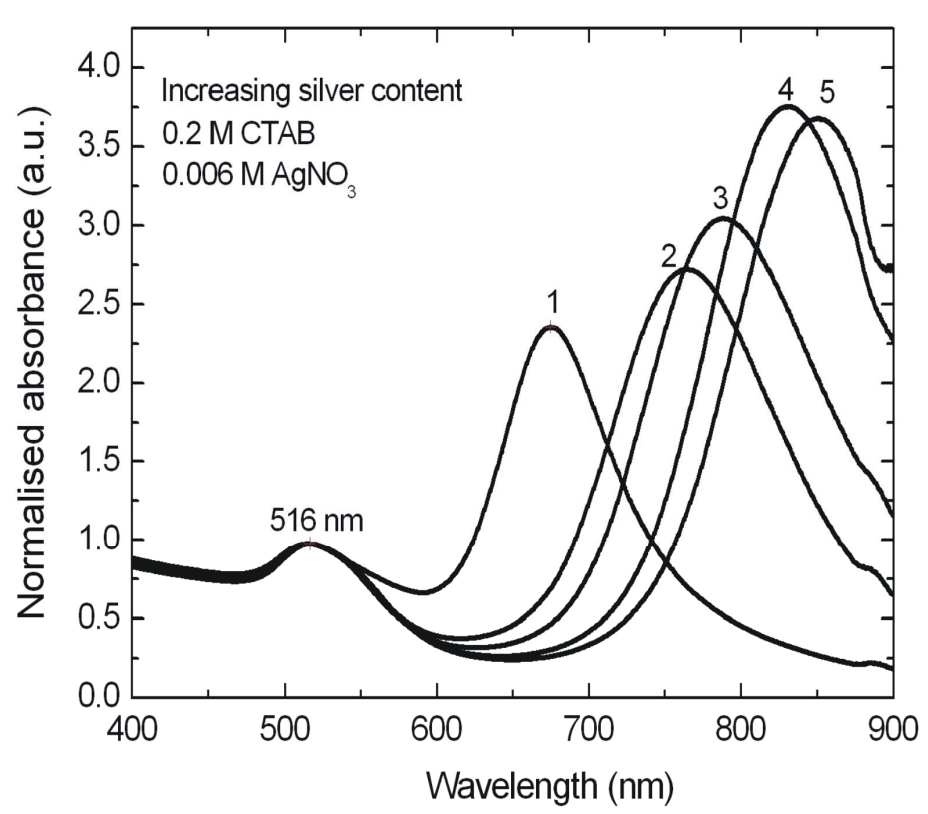

Figure 5: Normalized extinction spectra of gold nanorods with increasingly red-shifted longitudinal plasmon bands, synthesized using $50,100,150,200$, and $250 \mu \mathrm{l}$ of silver nitrate in the growth solution for curves 1-5, respectively. Normalization of the spectra is done with respect to the transverse plasmon peak amplitudes.

\section{Bioconjugation of gold nanospheres and gold nanorods}

A signature for successful binding of protein to gold NPs is a red-shifted and amplitude reduced plasmon band. Both these effects are due to the formation of the inhomogeneous layer of protein on the gold particle surface that leads to the modification of refractive index of the embedding medium. Figure 6(a) shows the extinction spectrum of nanospheres before and after incubation with HER81. Figure 6(b) is the corresponding situation in nanorods. In both cases, the characteristic red-shift in the extinction peak of the plasmon bands is seen.

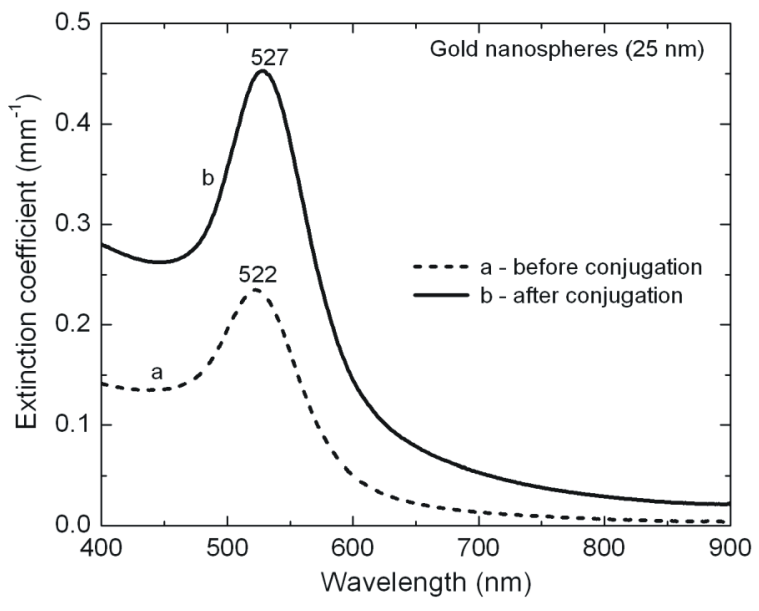

(a)

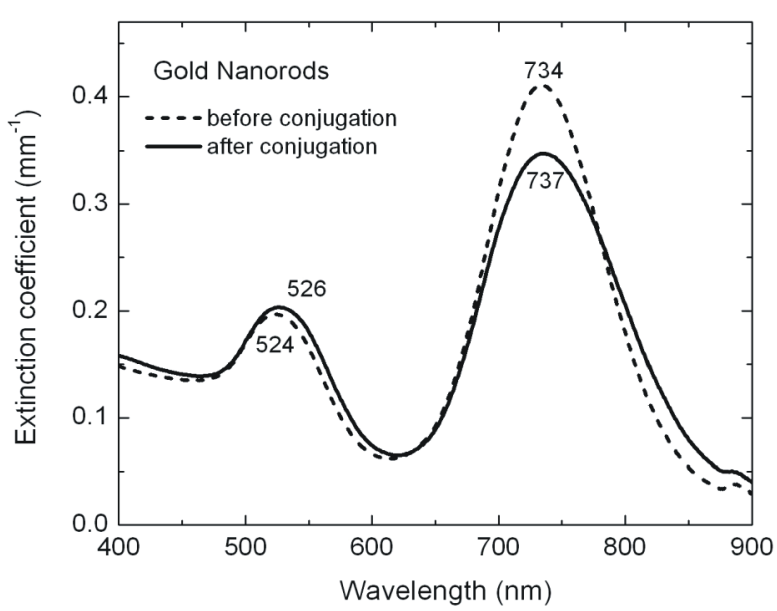

(b)

Figure 6: Extinction spectra before and after incubation of HER81 with (a) gold nanospheres, (b) gold nanorods. In both cases, a red shift in plasmon band(s) occurs after incubation with the antibody signifying successful bioconjugation. 
Not too much should be read into the amplitude changes of the extinction spectra since centrifugation of the bioconjugate to remove unbound protein, re-dispersion in water and other procedures result in a change in the concentration of the NPs used for spectroscopy. Figure 7(a) left image is the confocal reflectance image of the HER81/gold sphere conjugates incubated with SKBR3 cells. The right image of Figure 7(a) is the phase contrast image. As discussed in the experimental section, silver enhancement was used by which silver is reduced onto the gold particles forming large clusters around $500 \mathrm{~nm}$ in size. This then enables visualization under the microscope. The HER-2 receptors are expressed at the cell membranes of SKBR3 cells. The high intensities in both images at the cell membrane are then evidence of the preservation of the functionality of the antibody and illustrate successful conjugation. The images in Figure 7(b), which show the situation with the negative control using the $\mathrm{CHO}$ cells, display no such accumulation of gold particles.

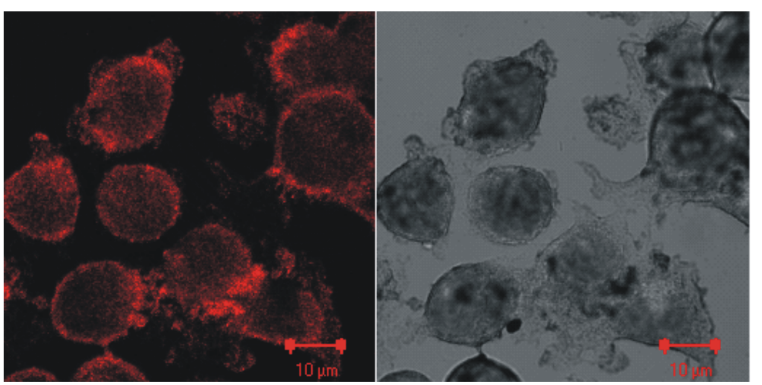

(a)

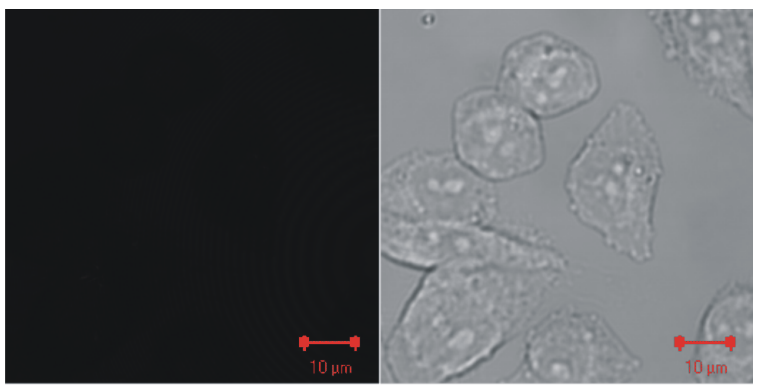

(b)

Figure 7: Confocal reflectance images (left) and bright field images (right) of (a) SKBR3 cells incubated with silver-stained HER81/gold sphere conjugates, (b) CHO cells under the same conditions. Care was taken to maintain the same acquisition parameters in both cases. The silver-stained bioconjugates are detected at the cell membranes of SKBR3 cells where HER2 is localized. This indicates successful conjugation and retention of functionality of the antibody after conjugation. No such accumulation of HER81/gold sphere conjugates is demonstrated in HER2 negative CHO cells.

Figure 8(a) and (b) are the results of the corresponding controls using the HER81/gold nanorods.

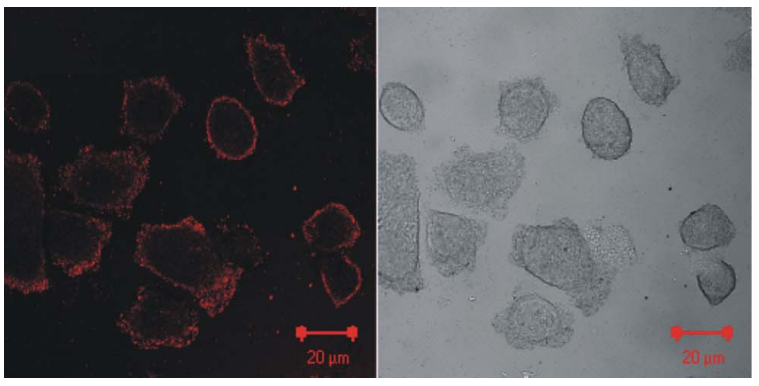

(a)

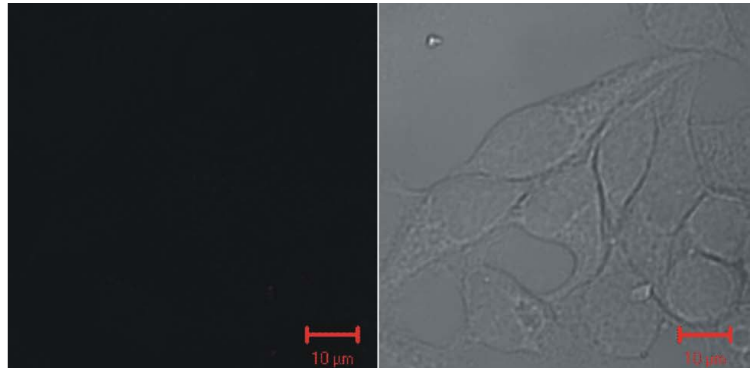

(b)

Figure 8: Corresponding images as in Figure 7 for bioconjugates consisting of silver-stained HER81/gold nanorod conjugates incubated with (a) SKBR3 cells and (b) CHO cells. Care was taken to maintain the same acquisition parameters in both cases. The bioconjugates are accumulated at the cell membranes of SKBR3 cells where HER2 is localized. As expected, no such accumulation takes place in the case of HER2 negative CHO cells. 


\section{Discussion}

\section{Gold nanorod synthesis}

The end products of the seed mediated growth protocols are crucially dependent on the nature of the seed: upon their size and upon the capping agents used. Additionally the constituents and their concentrations in the growth solution influence the outcome of the synthesis products. The addition of silver ions in the growth solution and the use of preformed CTAB stabilized seed in the protocol of Nikoobakht and El-Sayed [17] produced not only a high yield of monodisperse nanorods but fine tunability of aspect ratios.

There are many unanswered questions regarding the mechanism of formation of gold nanorods using the silver assisted protocol and this has been the topic of several studies [15, 17, 18, 19]. Recent reports of Orendorff and Murphy [19], and Liu and Guyot-Sionnest (23) provide some insights into the mechanisms that could be involved in the synthesis. It is postulated that silver ions are reduced by ascorbic acid even though it is a weak reducing agent, by the phenomenon of underpotential deposition (UPD). This is reduction of silver in monolayers on the growing gold nanorod surface at a potential less than the standard reduction potential [19]. The deposition is not uniform on the gold surface but occurs faster on the sidewalls compared with the end faces. Remarkably, the sidewalls in the case of nanorods produced with silver assistance using CTAB protected seed bear $\mathrm{Au}\{110\}$ faces, while the end faces have $\mathrm{Au}\{100\}$ faces. This is contrast to the rods prepared by using citrate-capped seed without $\mathrm{Ag}^{+}$. This faster passivation of the sidewalls is followed by CTAB binding possibly via bromide ions. This inhibits the reduction of gold, which deposits on the end faces. Ultimately, the end faces are also stabilized preventing the formation of very long nanorods. The model claims also to explain the increase in aspect ratio of the nanorods produced with higher concentration of silver ions used, by proposing that higher UPD of silver monolayers occur on the sidewalls which one assumes reduces the width of the nanorods thus increasing the aspect ratios [19].

Indeed, we observe some phenomena that are consistent with the above model. We are able to synthesize gold nanorods only up to an aspect ratio of 3.6 as shown in Figure 3. Addition of higher volumes of silver nitrate produces no further increase in the aspect ratios of the particles. These particles have an average length of $51 \mathrm{~nm}$. This supports the idea that ultimately complete passivation of the entire nanorod surface occurs preventing further gold 
deposition even though the reagents have not been exhausted. Further, we also observe that nanorods that are made with increasing $\mathrm{Ag}^{+}$volumes have smaller diameters with the lengths practically unchanged or only slightly increasing (See Table 1). The above model can also explain this. It must be mentioned that the model does not have an appealing explanation regarding the ability to tune the aspect ratios so precisely by $\mathrm{Ag}^{+}$variation. It is very likely that the model will have to undergo refinements or even major changes before it is universally accepted.

\section{Gold nanoparticle - antibody conjugation}

The non-covalent conjugation of proteins to colloidal gold is usually due to a combination of electrostatic and hydrophobic interactions. Citrate capped gold NPs are negatively charged due to a layer of negative citrate ions. Positively charged amino groups of the antibody will be attracted to the gold surface, and when the protein comes close enough for binding, the hydrophobic pockets of the protein will make contact and bind with the gold [24]. A general guideline to optimize the bioconjugation is that the $\mathrm{pH}$ of the antibody and the gold sol must be maintained at or slightly above the isoelectric point of the antibody [24].

Following the above procedures for citrate capped gold nanospheres with the HER81 antibody resulted in good bioconjugation as borne out by the spectroscopy and bioactivity studies on the positive control (Figure 7).

With the nanorods produced using the silver assisted protocol, the situation is more complex compared with nanospheres. In this case, the sidewalls are expected to be stabilized with a bilayer of CTAB, which imparts a positive charge to the gold. Huang et al [25], first changed the positively charged surface to a negatively charged one by exposing the nanorods to poly(styrenesulfonate) PSS polyelectrolyte solution. The PSS-capped nanorods were then treated in the same way as above with the conjugation being done with anti-EGFR monoclonal antibodies.

We performed a measurement of the zeta potential of the gold nanorod solution as originally prepared and determined a value of $+55 \mathrm{mV}$. Centrifugation to remove the excess unbound $\mathrm{CTAB}$ and re-dispersion of the rods in water saw a reduction in the zeta potential to $+7.5 \mathrm{mV}$, which also points to a low stability. We surmise that inspite of the net positive charge, the unpassivated end faces would be negatively charged due to the presence of $\mathrm{AuCl}^{-2}$ ions [21]. We therefore performed the same protocol as above and found that the bioconjugation indeed 
was achieved as borne out by the red-shifted extinction spectra as seen in Figure 6. Further, confocal microscopy successfully detected the bioconjugates in the positive control (Figure $8)$, indicating the success of the conjugation.

We believe that the mechanism of conjugation is the same as that in the case of gold nanospheres that is electrostatic and hydrophobic physisorption. It is also likely that at the $\mathrm{pH}$ at which the antibody is maintained, the Fc fragment of the antibody that is rich in positively charged amino side chains such as lysine will bind to the negatively charged chloride ion layer on the exposed end faces of the rods. We intend to perform studies that will elucidate this aspect. Further, we will perform the protocol of first capping the nanorods with PSS for example, and then comparing the antigen binding affinity constants of the bioconjugates from the two methods.

\section{Potential contrast enhancing applications}

The scattering and absorption bands of the synthesized nanorods span the wavelength regime between $675 \mathrm{~nm}-850 \mathrm{~nm}$ that is of interest to optical imaging. This occupies the most important part of the "optical imaging window" where light penetration in tissue is high due to reduced scattering and absorption coefficients. Optical imaging techniques (Table 2) that rely on scattering and /or absorption contrast to detect pathological tissue could benefit from the use of such nanoparticles with or without targeting capability.

\begin{tabular}{l|c|c|l|l}
\hline Technique & Imaging depth & Imaging resolution & \multicolumn{1}{|c}{ Mechanism } & Typical imaging applications \\
\hline Confocal microscopy [1] & $500 \mu \mathrm{m}$ & $>250 \mathrm{~nm}$ & Scattering /absorption & Tissue surfaces \\
\hline Two-photon microscopy [2] & $800 \mu \mathrm{m}$ & $>250 \mathrm{~nm}$ & Absorption & Tissue surfaces \\
\hline $\begin{array}{l}\text { Optical coherence } \\
\text { tomography [3] }\end{array}$ & $2 \mathrm{~mm}$ & $1 \mu \mathrm{m}$ & Scattering & $\begin{array}{l}\text { Surfaces/subsurfaces } \\
\text { of tissue }\end{array}$ \\
\hline $\begin{array}{l}\text { Diffuse optical tomography [4] } \\
\text { Photoacoustic imaging [5] }\end{array}$ & $>20 \mathrm{~mm}$ & $\approx 10 \%$ depth & Scattering/absorption & $\begin{array}{l}\text { Small animal; } \\
\text { human breast and brain }\end{array}$ \\
\hline
\end{tabular}

Table 2: Some important optical imaging techniques that utilize absorption and scattering contrasts in biology and medicine.

Our goal is to employ these particles as contrast agents for photoacoustic cancer imaging. Photoacoustic imaging relies on optical absorption for its signals. When photons are absorbed non-radiative de-excitation of the absorbed optical energy takes place with the release of localized heat. The local thermal expansion that results produces pressure transients [5]. When illuminated with pulsed laser light, a tumour site by virtue of its higher absorption with 
respect to the healthy background tissue, due to angiogenesis [26], will act as a source of bipolar photoacoustic pulses. This ultrasound propagates with minimal distortion to the surface where it is detected using appropriate wideband detectors. The time-of-flight, amplitude and peak-peak time of the bipolar PA pulse, possess information regarding the location, absorption and dimensions of the source, thereby permitting a reconstruction of the tumour site $[27,28]$.

It is known the NIR optical absorption contrast of tumours vis-à-vis healthy tissue, measured using optical mammographic methods, is between 1.5 and 3. Clinical trials of optical mammography are being conducted worldwide but at present, it seems implausible that intrinsic contrast alone will provide sufficient sensitivity and specificity and targeted contrast enhancement is likely to be required [29]. Since the same contrast mechanism of optical absorption is operative in photoacoustic imaging as well, a similar conclusion may be anticipated.

An impression of the feasibility of using the nanorods synthesized for contrast enhancement is now discussed The absorption cross-section of a nanorod at a wavelength say $800 \mathrm{~nm}$ is estimated using Discrete Dipole Approximation (DDSCAT) simulations [30, 31] as $C_{a b s}=2.8$ $\mathrm{x} 10^{-14} \mathrm{~m}^{2}$. A typical average optical absorption coefficient for an invasive ductal carcinoma is $\mu_{a}=0.008 \mathrm{~mm}^{-1}$ at $800 \mathrm{~nm}$. In order to achieve contrast enhancement, a certain number density of gold nanorods is required to exhibit higher absorption than the intrinsic value and may be calculated as:

$$
\rho_{\mathrm{NR}} \geq \frac{\mu_{a}}{C_{\mathrm{abs}}}
$$

This gives $\rho_{N R}=2.8 \times 10^{8} \mathrm{NR} / \mathrm{cm}^{3}$. Further photoacoustic signals can be enhanced by a thermal non-linearity mechanism to 3 orders of magnitude higher [32], then the modified number density of nanorods is only $\rho_{N R}=2.8 \times 10^{8} \mathrm{NR} / \mathrm{cm}^{5}$.

Published studies report that most tumor cell types express from $2 \times 10^{4}$ to $20 \times 10^{4}$ EGFR receptors/cell [12]. Let us assume arbitrarily that $2 \times 10^{3}$ of these sites per cell are occupied by conjugated nanorods. Further, if we assume that $1 \%$ of cells at a tumor site are diseased gives a figure of $2 \times 10^{6}$ cancer cells $/ \mathrm{cm}^{3}$. This will then lead to an estimation of the density of binding sites of the order of $10^{9} \mathrm{~cm}^{-3}$. Comparison of $\rho_{N R}$ and the estimated figure of density of binding sites leads us to believe that contrast enhancement will be possible. 
We will test these molecular probes in small animal photoacoustic imaging. We will start with simple tumour models that exhibit luxurious vascularisation in immuno compromised nude mice. One such model could be pancreas tumour cells (CA20948) injected subcutaneously or intramuscularly in the small animal. Further, models that are less vascularized will be used, such as prostate cancer tumours and sarcoma models. Photoacoustic imaging (with and without contrast agent) will be performed, with the animal anaesthetized using gaseous Isoflurane $/ \mathrm{O}_{2}$. The untargeted nanoparticles will be injected directly in the tumour in initial studies. Further, passive targeting will be studied by injecting the untargeted nanoparticles intravenously in the tail vein; enhanced permeation and retention (EPR) is expected to occur at the tumour site. Final studies will be based on active targeting, where the antibody coupled contrast agent will be injected in the tail vein.

In all studies, emphasis will be on ascertaining the sensitivity/efficacy of the technique with and without contrast agent. This will be done using calliper measurements and invasive endpoint methods such as excision and weighing of tumour masses, and immunehistopathology. The gold standard however will be contrast-enhanced MRI.

\section{Conclusions}

We have synthesized gold nanorods with optical extinction peaks in the region from $675 \mathrm{~nm}$ $850 \mathrm{~nm}$ making these eminently suited for scattering and absorption contrast enhancements in optical imaging. We have performed bioconjugation of these nanorods with HER81 antibodies, which have affinities for the HER-2neu receptors expressed by SKBR3 breast carcinoma cells. We demonstrated in fixated cell studies that the targeting functionality of the antibody moiety remains viable. However, it must be mentioned that the situation in vivo will be complex compared to the simplified situation in vitro. Other unresolved issues remain at present. One of these is regarding cell toxicity and cellular uptake of these particles in vivo. Further, whether these molecular probes will be able to extravasate into the tumor tissue through leaks in the vasculature has not yet been studied. These are some lines of research that we intend to follow in the near future.

\section{Acknowledgements}

We acknowledge fruitful discussions with Dr. Henk-Jan van Manen, Dr. Rolf Vermeij, and Dr. Christian Blum in various aspects related to bioconjugate chemistry, cell growth, and 
microscopy studies. The assistance of Sam Mathew (IIT Bombay) in early synthesis experiments is acknowledged. We thank Dr. Christina Graf (University of Wuerzburg) for discussions regarding the synthesis protocols. Peter van de Plas (Aurion, Wageningen, The Netherlands) is acknowledged for advice and tips on the use of bioconjugation protocols.

We received the HER81 mAb and SKBR3 cells from Prof. Leon Terstappen and Dr. Arjan Tibbe (Immunicon). Electron Microscopy was carried out by Mark Smithers and Dr. Enrico Keim (CMAL/MESA+). We thank Frank Roesthuis (LT/TNW) for the use of fume hoods in the clean room. The research is funded by the University of Twente through the thrust area program NIMTIK, and by the Nederlandse Wetenschappelijk Organisatie (NWO) and Stichting Technische Wetenschappen (STW) through project TTF 6527. Simulations of optical properties of particles were performed using supercomputing facilities of the National Computing Facilities Foundation (NCF) supported by the NWO.

\section{References}

1) J.A. Conchello and J. W. Lichtman, Nat. Meth., 2, pp. 920 - 931, 2005.

2) F. Helmchen and W. Denk, Nat. Meth., 2, pp. 932 - 940, 2005.

3) J. G Fujimoto, Nature Biotech., 21,pp 1361 1367, 2003.

4) D.A. Boas, D.H. Brooks, E.L. Miller; C.A. DiMarzio, M. Kilmer, R.J. Gaudette, Z. Quan, Signal Proc. Mag, IEEE, 18, pp.57-75, 2001.

5) M. Xu and L.V. Wang, Rev. Sci. Instrum., 77, 2006.

6) R.R. Kortom, and E. Sevick-Muraca, Annu. Rev. Phys. Chem., 47, pp. 555-606, 1996.

7) B.J. Tromberg, N. Shah, R. Lanning, A. Cerussi, J. Espinoza, T. Pham, L. Svaasand and J. Butler, Neoplasia, 2, pp. 26-20, 2000.

8) K. Licha, Contrast Agents II: Optical, Ultrasound, X-Ray and Radiopharmaceutical Imaging, 222, 2002.

9) X. Intes, J. Ripoll, Y. Chen, S. Nioka, A.G. Yodh, and B. Chance, Med. Phys., 30, pp. 1039-1047, 2003.

10) A.W.H. Lin, N.A. Lewinski, J.L. West, N.J. Halas, and R.A. Drezek, J. Biomed. Opt., 10, 064035, 2005.

11) X. Huang, I.H. El-Sayed, W. Qian and M.A. El-Sayed, J. Am. Chem. Soc., 128, pp. 2115 - 2120, 2006.

12) K. Sokolov, M. Follen, J. Aaron, I. Pavlova, A. Malpica, R. Lotan and R. Richards-Kortum, Canc. Res., 63, pp. 1999-2004, 2003.

13) D.A. Stuart, A.J. Haes, C.R. Yonzon, E.M. Hicks and R.P. Van Duyne, IEEE Proc.-Nanobiotechnol, 152, pp. 13-32, 2005.

14) A. Gole, and C.J. Murphy, Chem. Mater., 16, pp. 3633 -3640, 2004.

15) C.J. Murphy, T.K. Sau, A.M. Gole, C. J. Orendorff, J. Gao, L. Gou, S. E. Hunyadi and T. Li, J. Phys. Chem. B, 109, pp. 13857-13870, 2005.

16) N.R. Jana, L. Gearheart and C.J. Murphy, Adv. Mater, 13, pp 1389-1393, 2001.

17) B. Nikoobakht and M.A. El-Sayed, Chem. Mater, 15, pp. 1957-1962, 2003.

18) T.K. Sau and C.J. Murphy, Langmuir, 20, pp. 6414 -6420, 2004.

19) C.J. Orendorff, and C.J. Murphy, J. Phys. Chem. B., 110, pp. 3990-3994, 2006.

20) D.J. Slamon, G.M. Clark, S.G. Wong, W.J. Levin, A. Ullrich and W.L. McGuire, Science, 235, pp. 177-182, 1987.

21) D.A. Handley, Colloidal Gold: Principles, Methods, and Applications (Hayat, M. A., ed), pp. 13-32, Academic Press, Inc., New York, 1989.

22) B. Nikoobakht and M.A. El-Sayed, Langmuir, 17, pp. 6368-6374, 2001.

23) M. Liu and P. Guyot-Sionnest, J. Phys. Chem. B., 109, pp. 22192-22200, 2005.

24) G.T. Hermanson, Bioconjugate techniques, pp. 593-605, Academic Press, Inc., New York, 1996. 
25) X. Huang, I.H. El-Sayed, and M.A. El-Sayed, J Am. Chem. Soc, 125, pp. 1215-1220, 2006.

26) P. Carmeliet and R. K. Jain, Nature, 407, pp. 249-257, 2000.

27) S. Manohar, A. Kharine, J. C. G. van Hespen, W. Steenbergen, and T. G. van Leeuwen, J. Biomed. Opt., 9, pp. 1172-1181, 2004.

28) S. Manohar, A. Kharine, J. C. G. van Hespen, W. Steenbergen, and T. G. van Leeuwen, Phys. Med. Biol, 50, pp. 2543-2557, 2005.

29) H. Rinneberg, D. Grosenick, K. T. Moesta, J. Mucke, B. Gebauer, C. Stroszczynski, H. Wabnitz, M. Moeller, B. Wassermann and P.M. Schlag, Tech. Cancer Res. Treat, 4, 2005.

30) B.T. Draine and P.J. Flatau, http://arxiv.org/abs/astro-ph/xxx.

31) B.T. Draine and P.J. Flatau, J. Opt. Soc. Amer., 11A, pp. 1491-1499, 1994.

32) I.G. Calasso, W. Craig and G.J. Diebold, Phys. Rev. Lett., 86, pp. 3550-3553, 2001. 


\title{
Chapter 3
}

\section{Iodide Impurities in Hexadecyltrimethylammonium Bromide (CTAB) Products: Lot-Lot Variations and Influence on Gold Nanorod Synthesis}

\begin{abstract}
Recent reports [Smith and Korgel, Langmuir 2008, 24, 644-649 and Smith et al., Langmuir 2009, 25, 9518-9524] have implicated certain hexadecyltrimethylammonium bromide (CTAB) products with iodide impurities, in the failure of a seed-mediated, silver and surfactant-assisted growth protocol, to produce gold nanorods. We used two of the three 'suspect' CTAB products and a 'good' CTAB product in the protocol, varying silver nitrate solutions in the growth solutions. We obtained excellent gold nanorod samples as witnessed in signature longitudinal plasmon peaks in optical extinction spectra, which we substantiated using electron microscopy. Analysis of these samples using inductively coupled plasma mass spectroscopy (ICP-MS) failed to detect iodide. We subsequently learnt from discussions with Smith et al. that different lot numbers within the same product had been analyzed by our respective laboratories. We can conclude that iodide impurities can vary significantly from lot to lot within a product, to such an extent that there is no guarantee that gold nanorods can be synthesized with one or other CTAB product. Conversely, labeling a CTAB product, identified by a product number or supplier name, as one whose use precludes the formation of nanorods, is also hasty.
\end{abstract}

This chapter has been published in Langmuir as "R.G. Rayavarapu, C. Ungureanu, P. Krystek†े, T.G. van Leeuwen and S. Manohar" (†- MiPlaza, Philips Research). 


\section{Introduction}

Rod-shaped gold nanoparticles have attracted intense attention from researchers largely on two fronts:

1. in the biomedical physics arena where predominantly the plasmon resonance-driven optical features, especially intense absorptions in the near-infrared, inspire new ideas for applications in molecular medicine [1-6].

2. in the fundamental chemistry and physics of underlying mechanisms of methods that initiate and nurture symmetry breaking of gold nuclei to form rods [7-16].

This understanding in 2. is constantly evolving but as yet is not complete for synthesis protocols which may be described as working fairly well in their control of the size and shape of the nanoparticle products $[8,10,11,17]$. Basic scientific curiosity as to the mechanics at the atomic and molecular scales that culminate in the rod-shaped particles is not the only driving force for this research. A good understanding will provide a handle towards the desired exquisite and reproducible control over nanoparticle sizes and shapes that will accelerate the transition of certain synthetic routes from laboratory protocols to manufacturing processes.

Recently the group of Smith and Korgel [18] published a study on the dependence of the success or failure of a well-accepted gold nanorod synthesis protocol $[10,19]$ on the source of hexadecyltrimethylammonium bromide (CTAB) used in the experiments. This study was initiated following their discovery $[18,20]$ that certain $\mathrm{CTAB}$ products resulted only in nanosphere formation, while other products yielded nanorods as expected. The paper concluded that there was an undetermined impurity in certain $\mathrm{CTAB}$ products that disrupted the mechanism that produced nanorods. This impurity was subsequently identified as being iodide using inductively coupled plasma mass spectroscopy (ICP-MS) [21].

This intrigued us since we had used different CTAB products in synthesizing nanorods, always successfully [22]. We decided to test two of the three 'wrong' or 'suspect' CTABs (the third was not available from the supplier anymore) and a 'good' CTAB: Acros 22716V, Sigma H5882 and Fluka 52370 respectively. The synthesis procedure we used was similar to that described in Refs. 18 and 21. We found that we were able to synthesize excellent samples of nanorods from all three CTAB products. We then carefully analyzed the samples 
using inductively coupled plasma mass spectroscopy (ICP-MS), with methodological limits of detection superior to those in the aforementioned reports. No significant amounts of iodide were detected in any of the CTAB products.

\section{Experimental}

\section{Protocol}

We used, to the best of our understanding, the same protocol followed in Smith and Korgel [18] and Smith et al [21]. The only exception is that we use the growing gold spheres in the growth solution within 5 minutes, while Ref. 19 seeds growth solution after 2 hours. However, we do not believe this to be a crucial difference. The method is from Ref. 19 which itself is based on the seed-mediated silver-CTAB assisted protocol of Nikoobakht and ElSayed [10]. We describe here the steps followed:

1. Preparation of growth solution: To freshly prepared $0.5 \mathrm{ml}$ of $0.01 \mathrm{M}$ gold salt solution, is added $9.5 \mathrm{ml}$ of $0.1 \mathrm{M} \mathrm{CTAB}$ solution with thorough mixing to yield a dark yellow solution. To this $55 \mu \mathrm{l}$ of $0.1 \mathrm{M}$ ascorbic acid is added with stirring. The resultant turns colorless. According to Ref. 19, a volume of between 20 - $100 \mu 1$ of $0.01 \mathrm{M}$ silver nitrate $\left(\mathrm{AgNO}_{3}\right)$ may be added to make the growth solution, but the Korgel group [19] appears to have specifically used $75 \mu \mathrm{l}$. We decided to use different volumes of $0.01 \mathrm{M} \mathrm{AgNO}_{3}(20,50,70,100,200,250$, 300 and $350 \mu \mathrm{l}$ ), thus making 8 growth solutions which would be seeded in a subsequent step.

2. Preparation of gold seed: To freshly prepared $0.25 \mathrm{ml}$ of $0.01 \mathrm{M}$ gold salt solution, $9.75 \mathrm{ml}$ of $0.1 \mathrm{M} \mathrm{CTAB}$ is added with stirring. Ice-cold, freshly prepared $0.01 \mathrm{M}$ sodium borohydride solution in a volume of $0.6 \mathrm{ml}$ is added to the mixture all at once with vigorous stirring for 2 minutes. The resultant is used within about 5 minutes to seed each growth solution.

3. Growth phase: The seed solution is added in a volume of $12 \mu 1$ to the growth solutions with gentle stirring. The resultants are maintained undisturbed at $25^{\circ} \mathrm{C}$ for 24 hours after which they are centrifuged and the supernatants removed. The precipitates are re-dispersed in $10 \mathrm{ml}$ of Milli Q water.

This procedure was repeated for each CTAB product tested. 


\section{Deliberate contamination of good CTAB with potassium iodide}

The protocol described above was followed using Fluka 52370 (see further) with the differences that two different volumes of $0.1 \mathrm{M}$ potassium iodide (KI) were added to the growth solutions to obtain end concentrations of $0.86 \mu \mathrm{M}$ and $1.72 \mu \mathrm{M} \mathrm{KI}$.

\section{Materials and supplies}

We used the following CTAB products: Acros 22716V, Sigma H5882 and Fluka 52370, for both growth and seed solutions. Of these the first two were 'wrong' $[18,21]$ or 'suspect' CTAB, while the third was a 'good' CTAB.

Gold salt (Tetrachloroauric acid $\mathrm{HAuCl}_{4} \cdot 3 \mathrm{H}_{2} \mathrm{O}, 99.99 \%$ ) was purchased from Acros Organics (Belgium), sodium borohydride $\left(\mathrm{NaBH}_{4}, 99 \%\right)$, and ascorbic acid (99\%) from Aldrich (The Netherlands), and silver nitrate $\left(\mathrm{AgNO}_{3}, 99.8 \%\right)$ from Merck (Germany). Solutions were prepared using Milli-Q Gradient System water (Millipore, QuantumR-EX). Prior to use, all glassware was cleaned with hydrofluoric acid $(\mathrm{HF})$, further with aquaregia $\left(\mathrm{HCl} / \mathrm{HNO}_{3}\right)$ and rinsed thoroughly with Milli Q water.

\section{Materials characterization using ICP-MS}

In addition to the CTAB samples above, we also studied Sigma H9151 as a second 'good' sample. The CTAB powders were weighed to $0.03 \mathrm{~g}$ in duplicate, and dissolved in $6 \mathrm{ml} 2 \%$ nitric acid $\left(\mathrm{HNO}_{3}\right)$. To ensure dissolution of the samples, the vessels were placed in an ultrasonic bath for 15 minutes. The samples were then diluted 10-fold in an organic alkali: $2 \%$ tetra methyl ammonium hydroxide (TMAH) or tetraethyl ammonium hydroxide (TEAH) both from Fluka, were tested. Indium (In) was added off-line as the internal standard for drift compensation during the ICP-MS measurements. The final concentration in the analyzed solutions was $0.5 \mathrm{ppm}$ In. The determination of iodide was carried out using a Perkin Elmer Elan 6100 DRC II ICP-MS.

The following operating conditions were used:

Forward RF power: $1450 \mathrm{~W}$; Plasma gas flow: $15 \mathrm{~L} / \mathrm{min}$ Ar; Auxiliary gas flow: $1.13 \mathrm{~L} / \mathrm{min}$ Ar; Nebulizer gas flow: $0.90 \mathrm{~L} / \mathrm{min}$ Ar. The isotopes ${ }^{127} \mathrm{I}$ and ${ }^{115} \mathrm{In}$ were measured using an integration time of $4.5 \mathrm{~s}$, for five replicates. The calibration solutions were prepared from a stock standard solution from Perkin Elmer in the calibration range 0.25 to $1000 \mathrm{ppb}$ I. 


\section{Nanoparticle characterization}

Electron microscopy of the nanoparticles was performed using a Zeiss-1550 scanning electron microscope (SEM). Particle sizes were estimated using the NI Vision module (Labview, National Instruments) on the digital SEM images with around 200 particles considered in each case. In cases where the numbers of particles (rods or spheres) were low, the sizes were estimated using the available numbers which are mentioned where size statistics are presented (See Supporting Information). Optical transmission spectra ( $T$ vs. 1) of nanoparticles were measured with collimated transmission in a Shimadzu PC3101 UV-VisNIR spectrophotometer. Using the Beer-Lambert law, these were converted to extinction $\left(\mu_{e x t}\right.$ $\left.\left[\mathrm{mm}^{-1}\right]\right)$ values as:

$$
\mu_{e x t}(\lambda) \mid=\frac{1}{d} \ln \left\{\frac{T(\lambda)}{100}\right\}
$$

where $d[\mathrm{~mm}]$ is the pathlength of the cuvettes used.

With the size and shape of the particles, ascertained from SEM and using Mie theory or Discrete Dipole Approximation (DDA) [23, 24] for spheres and rods respectively, the optical extinction efficiency $\left(Q_{e x t}\right)$ of the nanoparticles was calculated. The concentration of particles was calculated as (See Supporting Information):

$$
N_{\text {part }}\left[\mathrm{cm}^{-3}\right]=\frac{\mu_{\mathrm{ext}}\left[\mathrm{cm}^{-1}\right]}{\mathrm{Q}_{\mathrm{ext}} \pi \mathrm{r}^{2}\left[\mathrm{~cm}^{2}\right]}
$$

where $r=(3 V=4 \pi)^{1 / 3}$ is the effective radius of a sphere having the same volume $(V)$ as the particle.

\section{Results}

\section{Nanoparticles using Acros 22716V CTAB}

Figure 1(a) shows the optical extinction spectra of the 8 samples prepared using the different volumes of $0.01 \mathrm{M} \mathrm{AgNO}_{3}$. Each spectrum's peak at $522 \mathrm{~nm}$ corresponds to the single plasmon peak of gold nanospheres and the transverse plasmon peak of gold nanorods. Each curve's reddened peak is the signature longitudinal plasmon peak of gold nanorods, incidentally the biggest reason for the enormous interest in these particles for applications in molecular medicine as contrast agents for light [25] or light-excited therapeutic agents [3]. For the case of $20 \mu \mathrm{l} \mathrm{ANO}_{3}$, the relative amplitudes of the two peaks indicate that the sol 
contains a predominance of nanospheres compared with nanorods. From $50 \mu \mathrm{AgNO}$ onwards, the situation is reversed with increasingly higher concentrations of nanorods. Beyond $300 \mu \mathrm{AgNO}_{3}$, the red-shifting of the longitudinal plasmon peak and the increase in its amplitude appear to stop at $950 \mathrm{~nm}$, with the last spectrum showing a slightly blue-shifted and lower amplitude peak.

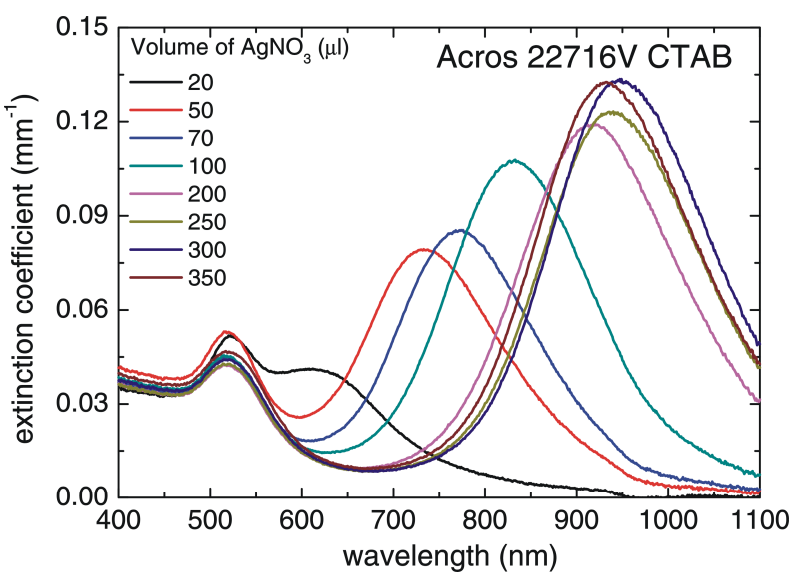

(a)

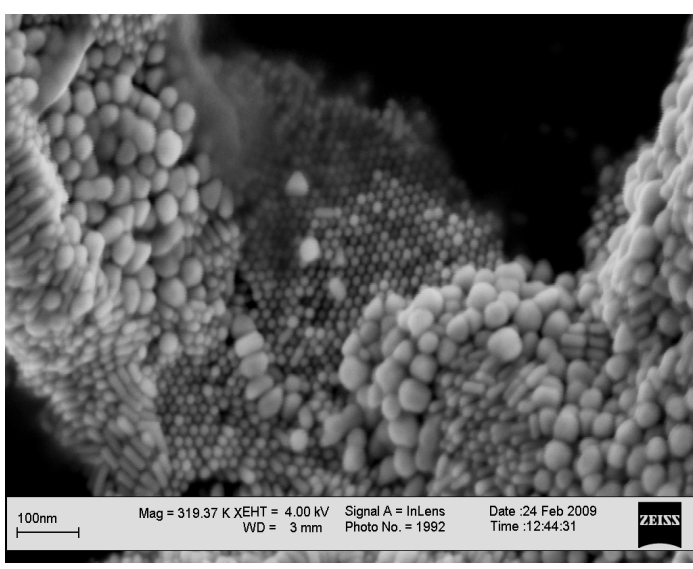

(b)

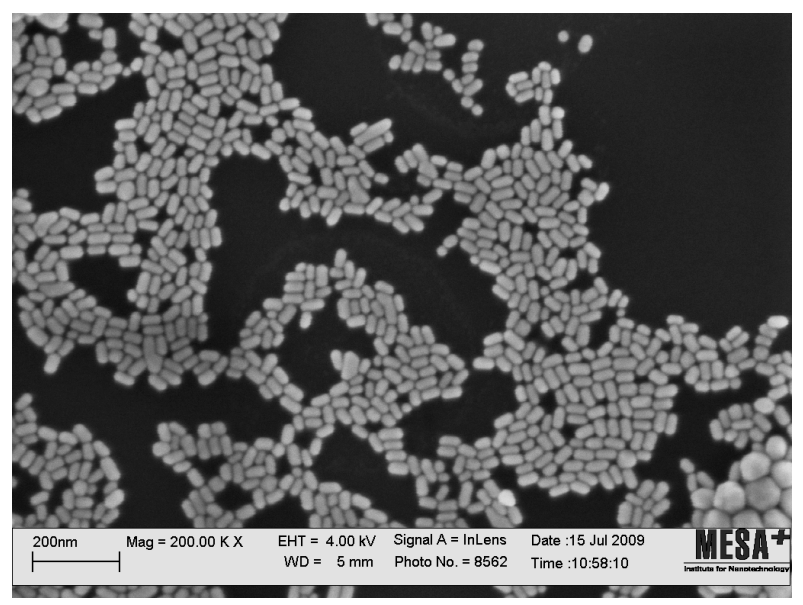

(c)

Figure 1: (a) Optical extinction spectra of gold nanoparticles synthesized when Acros 22716V CTAB was used. Red-shifted peaks in each spectrum indicate the presence of nanorods; all cases studied yielded nanorods with this CTAB. Scanning electron micrographs (SEM) of gold nanoparticles prepared when (b) $20 \mu 1$ and (c) $50 \mu \mathrm{l}$ of $0.01 \mathrm{M} \mathrm{AgNO}_{3}$ were used respectively. In the former case, a relatively higher percentage of nanospheres are produced.

Figures 1(b) and (c) are the SEM images of the particles produced using $20 \mu 1$ and $50 \mu 1$ $\mathrm{AgNO}_{3}$. With the lowest volume of $\mathrm{AgNO}_{3}$, there are indeed a higher proportion of spherical particles compared with nanorods. 


\section{Nanoparticles using Sigma H5882 CTAB}

The extinction spectra in Figure 2 show the two signature peaks that betray gold nanorod presence, in all but the $20 \mu \mathrm{l} 0.01 \mathrm{M} \mathrm{AgNO}_{3}$ case; the solitary peak is due to gold nanospheres and no nanorods have been formed.

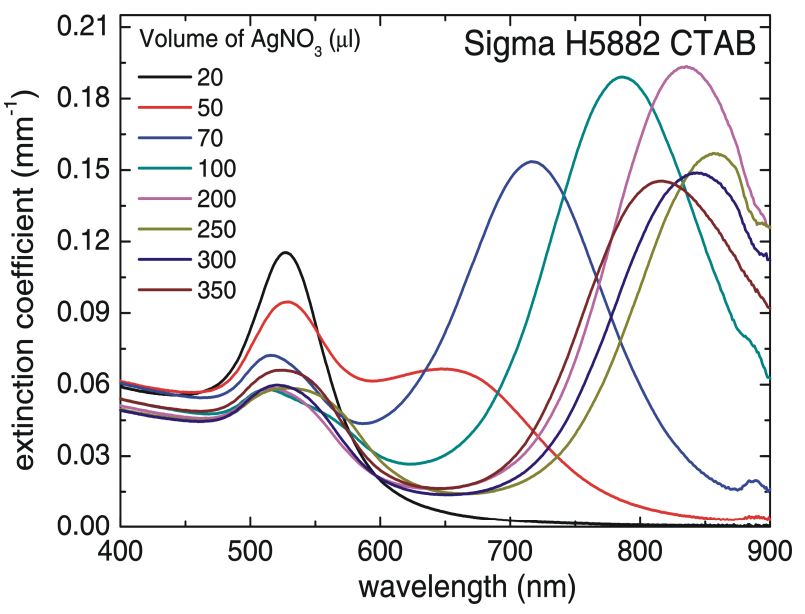

(a)

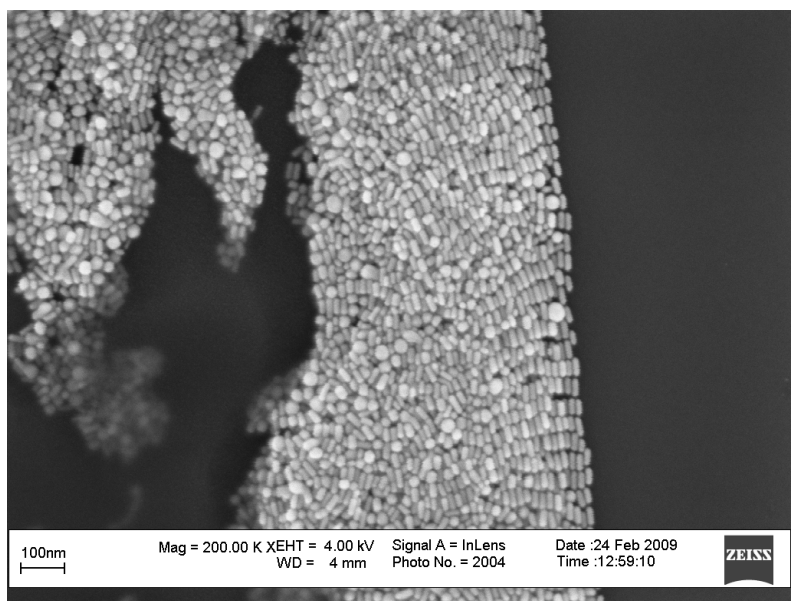

(c)

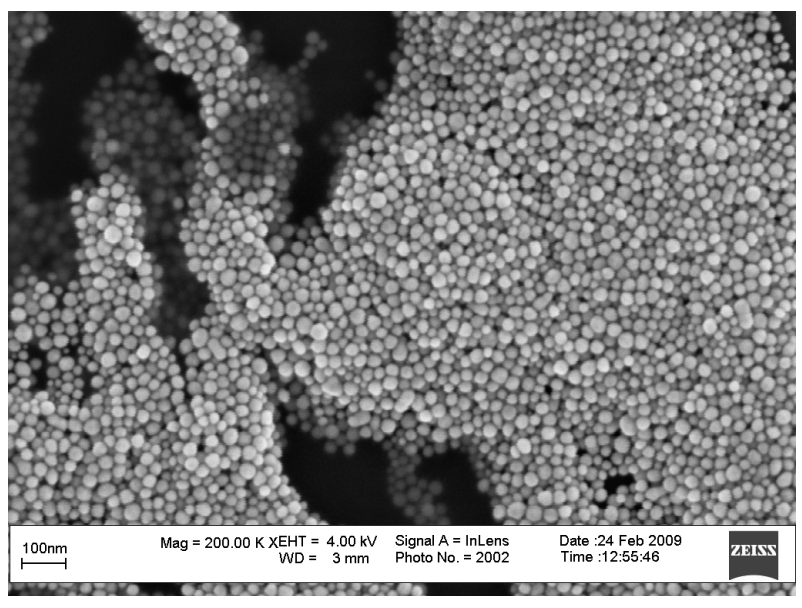

(b)

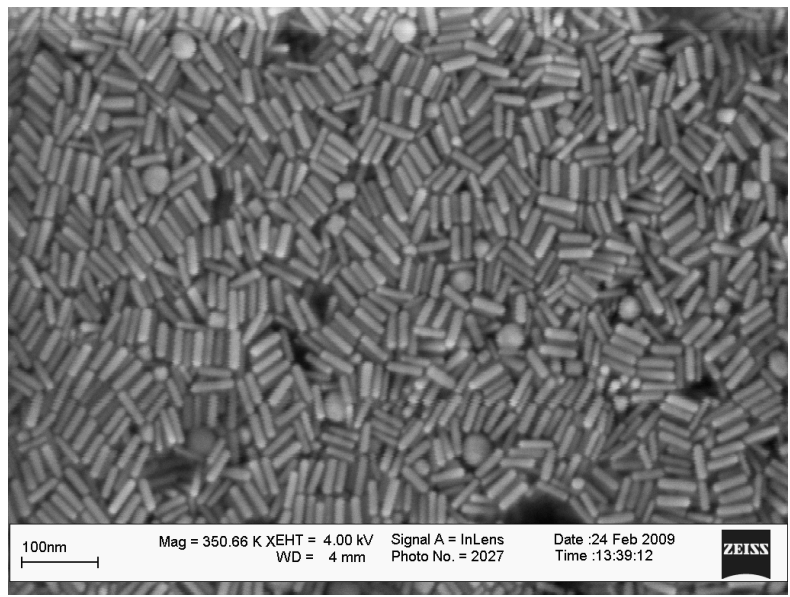

(d)

Figure 2: (a) Optical extinction spectra of gold nanoparticles synthesized when Sigma H5882 CTAB was used. Only for the case when $20 \mu \mathrm{l}$ of $0.01 \mathrm{M} \mathrm{AgNO}_{3}$ was used in the growth solution no nanorods were produced, as seen in the solitary peak at $522 \mathrm{~nm}$ signifying the presence of nanospheres. Scanning electron micrographs (SEM) of gold nanoparticles prepared with (b) $20 \mu \mathrm{l}$ of $\mathrm{AgNO}_{3}$ where only nanospheres were produced; (c) 50 $\mu \mathrm{l}$ of $\mathrm{AgNO}_{3}$ where a relatively high percentage of nanospheres compared with nanorods were produced, and (d) $250 \mu \mathrm{l}$ of $\mathrm{AgNO}_{3}$ where a high percentage of nanorods were produced.

With the use of $50 \mu \mathrm{AgNO}_{3}$ a relatively higher proportion of gold spheres are present, but with progressively increasing $\mathrm{Ag}^{+}$ion concentrations, nanorods are formed in abundance. These are seen to possess higher aspect ratios with the red-shifting of the plasmon peaks until $850 \mathrm{~nm}$ for $250 \mu \mathrm{l} \mathrm{AgNO}_{3}$. Beyond this, there is regression with blue-shifting and lowering of amplitudes. The SEM images corresponding to the cases 20,50 and $250 \mu 1 \mathrm{AgNO}_{3}$ are seen 
in Figure 2(b), (c) and (d) which corroborate the spectral evidence for the relative absence or presence of gold nanorods in the three cases.

\section{Nanoparticles using Fluka 52370 CTAB}

The extinction spectra shown Figure 3 show a behavior quite similar to the case with Acros, where nanorods are produced for all volumes of $0.01 \mathrm{M} \mathrm{AgNO}_{3}$ used. Red-shifting however stops at $850 \mathrm{~nm}$ for $200 \mu \mathrm{AgNO}_{3}$. It should be noted that the optical densities, which correspond to the concentrations of particles in the sol, are higher in this case compared with when the Acros and Sigma CTAB products are used.

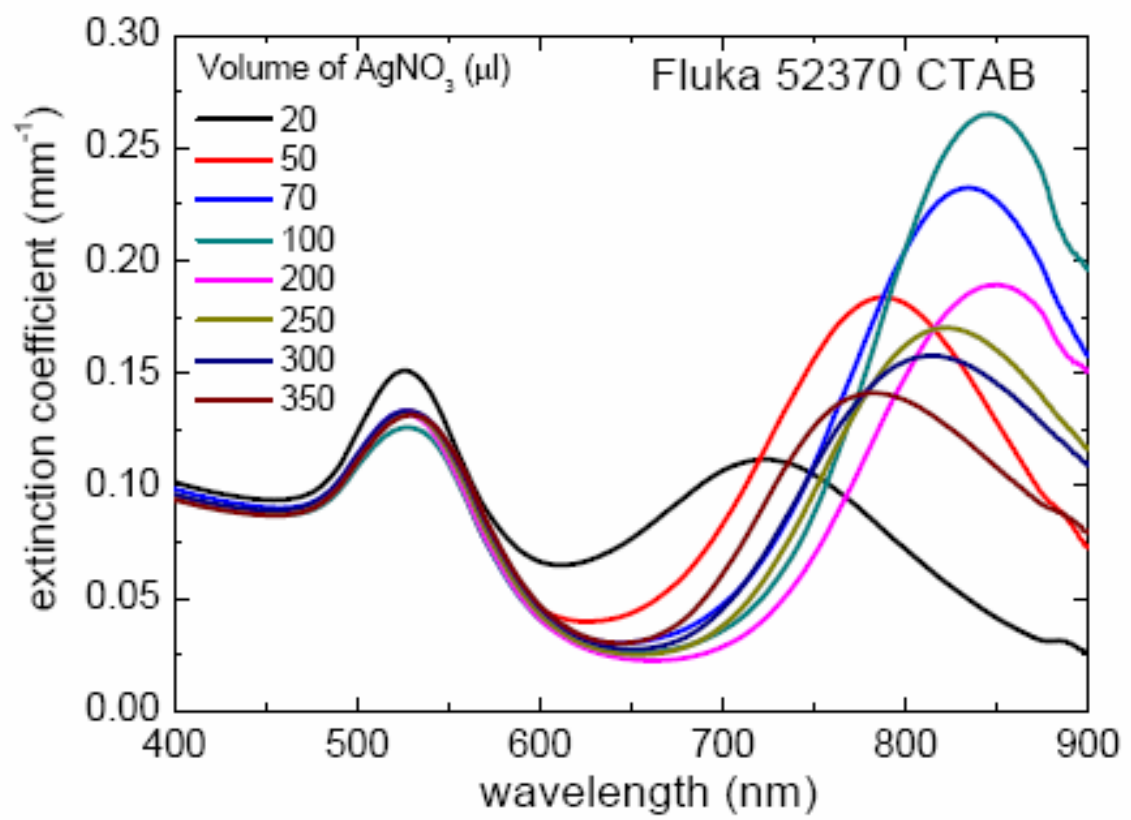

Figure 3: Optical extinction spectra of gold nanoparticles synthesized when Fluka 52370 CTAB was used. In all cases studied, nanorods were produced with this CTAB.

\section{ICP-MS analysis of CTAB products}

The results of several quality control experiments are summarized:

- Control of calibration range: The concentration of $25 \mathrm{ppb}$ was controlled and the recovery was $(n=1) 101 \%$. The concentration of $100 \mathrm{ppb}$ was controlled and the recovery was $(\mathrm{n}=1) 117 \%$.

- Control of reference substance: Potassium iodide (KI) was used as reference substance. Two samples were pre-treated and measured according to the same procedure. The recovery was $(n=2) 92.5 \pm 0.5 \%$. 
- Standard addition experiments to the CTAB matrix: Two recovery experiments by standard addition of a known concentration of iodide to the CTAB matrix were carried out and the recovery was $(n=2) 101 \pm 6 \%$.

\section{Other tested variations of the procedure}

Two experiments of the CTAB matrix were carried out by using a higher sample amount of 0.1 g. Nevertheless, this did not lead to significantly different results. Also the use of different organic alkalis (TMAH as well as TEAH) did not lead to significantly different results.

The methodological limit of detection (based on a weight of $0.03 \mathrm{~g}$ CTAB) was determined to be $0.5 \mathrm{ppm}$ In. In all samples, whether 'good' or 'suspect', no significant amounts of iodide were detected.

\section{Nanoparticles using Fluka 52370 CTAB deliberately contaminated with potassium iodide}

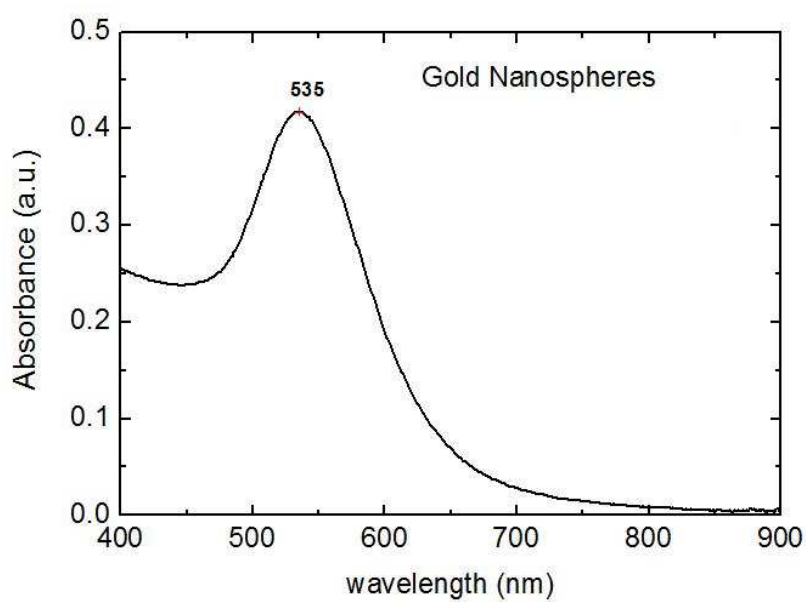

(a)

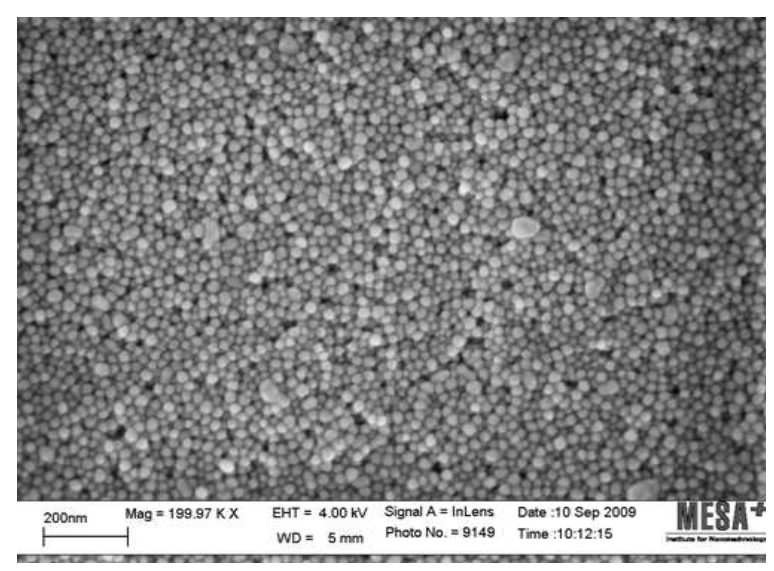

(b)

Figure 4: For nanoparticles generated using growth solutions carrying $1.72 \mu \mathrm{M}$ potassium iodide in the growth solutions. (a) Optical extinction spectrum showing the solitary plasmon features indicative of gold nanospheres and not nanorods, (b) Scanning Electron Microscopy (SEM) image of the particles confirming the nonformation of nanorods.

Figure 4(a) shows the optical extinction spectrum for the particles produced with KI impurity in a concentration of $1.72 \mu \mathrm{M}$ in the growth solution. The SEM image of the resulting particles is shown in Figure 4(b). Only gold spheres are obtained in this case. Similar results are obtained with $\mathrm{KI}$ concentration of $0.86 \mu \mathrm{M}$ in the growth solution (not shown). These results are in agreement with the report of Smith et al [21]. 


\section{Discussion}

We have synthesized excellent samples of gold nanorods using all three CTAB products including the two 'suspect' ones as shown in electron microscopy images and the optical spectra. The optical spectra may be described as being textbook curves with sharp peaks marking the wavelengths where the excited plasmons resonate along the length and width of the rod-shaped nanoparticles. The great sensitivity that nanorod sizes and consequently longitudinal plasmon peak positions, have for the $\mathrm{Ag}^{+}$ion concentrations in the growth solution are also evident (see Figures 1(a), 2(a) and 3) using the different CTAB products. We have no doubt that the same physicochemical mechanisms responsible for the symmetry breaking in gold seed evolution and growth into nanorods dominate for all CTABs used with no evidence of disruption of the nanorod formation due to any impurities. In sharp contradiction to Smith et al [21], we were unable to detect iodide impurities in the 'suspect' products. This finding concurs with the success of the nanorod synthesis protocol, when these CTAB products were used. When we deliberately contaminated the products with iodide, gold nanorod formation was indeed disrupted (figure 4) corroborating the results of Smith et al [21]. We are confident of the accuracy and reliability of our ICP-MS measurements, which were performed with care after ensuring that several quality control checks had been successfully carried out. From discussions with the authors of Ref. 16 and 19, we learnt that the lot numbers analyzed by our two laboratories were different within the same CTAB product number. Table 1 consolidates our experiences and those of Smith et al [21] with the various $\mathrm{CTAB}$ products. It is clear that there are significant variations in the presence of iodide impurities between the lots of the two 'suspect' CTABs, which implies that that the samples quantified by Smith et al [21] may be considered as different from the samples we studied even under the same product name.

\begin{tabular}{ccccccccc}
$\begin{array}{c}\text { supplier } \\
\text { and purity }\end{array}$ & $\begin{array}{c}\text { product } \\
\text { number }\end{array}$ & \multicolumn{2}{c}{ lot numbers } & \multicolumn{2}{c}{ nanorods } & \multicolumn{2}{c}{ [I](ppm) } \\
& & ref.21 & this work & ref.21 & this work & ref.21 & this work \\
Acros $\geq 99 \%$ & 22716 & B0116374 & A0258881 & no & yes & 57.68 & $<0.5$ \\
Sigma $\geq 99 \%$ & H5882 & 055K0140 & 117K0732 & no & yes & 839.27 & $<0.5$ \\
Fluka $\geq 96 \%$ & 52370 & $445709 / 110703248$ & 43608036 & yes & yes & $<2.75$ & $<0.5$ \\
Sigma $\geq 96 \%$ & H9151 & 095K0187 & $018 K 35291$ & yes & yes & $<2.75$ & $<0.5$
\end{tabular}

Table 1: Overview of results of gold nanorod synthesis, from this work and from Ref. 21, using various CTAB products with their iodide impurity concentrations measured using ICP-MS. The lot numbers of products used in Ref. 21 was obtained from the authors of the article. 
It should be mentioned that even when nanorods are successfully synthesized, size reproducibility, manifested in variations in the optical properties across the CTAB products we studied leaves much to be desired.

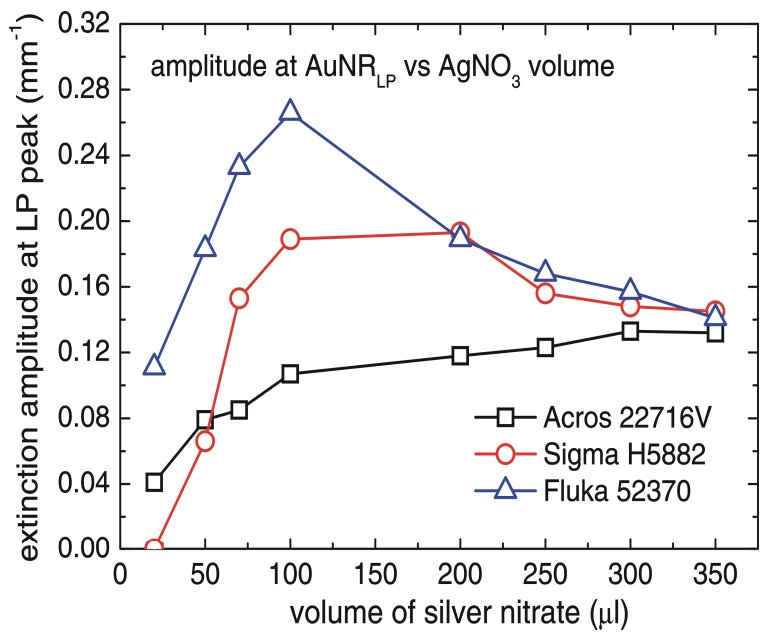

(a)

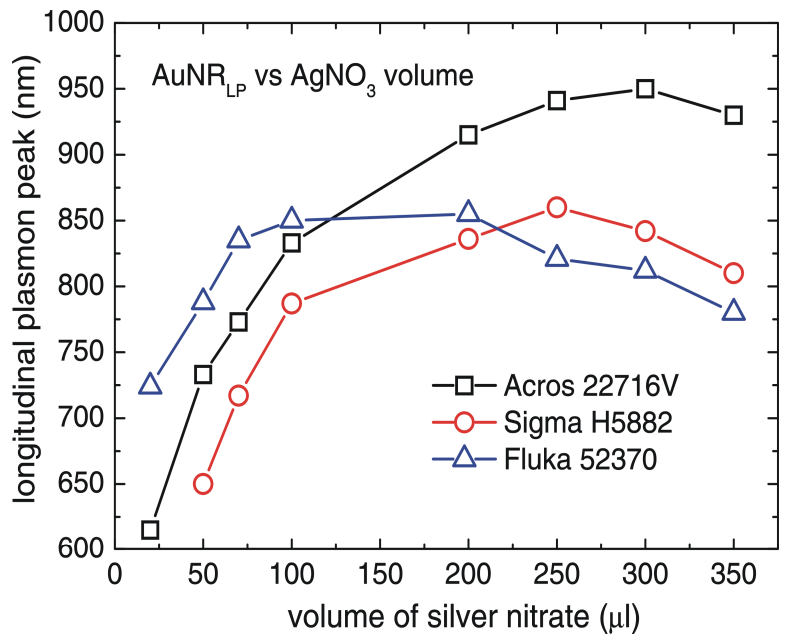

(b)

Figure 5: Variation with respect to the volume of $\mathrm{AgNO}_{3}$ used in the growth solutions of (a) the amplitude of the extinction coefficient at the longitudinal plasmon peak, and (b) the position of the longitudinal plasmon peak.

Figure 5(a) and (b) consolidate the outstanding differences between the nanorods produced using the various CTABs. An important difference is the threshold volumes of $\mathrm{AgNO}_{3}$ required to produce nanorods. In the case with Sigma H5882, no nanorods are formed using $20 \mu \mathrm{AgNO}_{3}$, in contrast with the other 2 cases. Further, the amplitudes of the longitudinal plasmon peaks (Figure 5(a)) and their positions (Figure 5(b)) are different in the 3 cases. It is possible that these variations are due to the modulation of the basic nanorod formation mechanism, by an interfering process as explained by Smith et al [21] due to the presence of iodide or other trace impurities, which in our case are below the detectable limits. Also, while utmost care was taken, it is not possible to exclude variations in environmental conditions such as in ambient temperature etc, uncertainties in experimental conditions such as in seed aging times, in concentrations of components taken etc which could be affecting various steps in the trajectory followed for making the nanorods. These uncertainties would propagate in complex ways, which could affect the final results. One is also encouraged to resort to tweaking concentrations of various products added or fine-tune certain experimental conditions, to get the nanorod products that are desired. This is exemplified by a hypothetical situation, where had we stayed with the case of $20 \mu \mathrm{AgNO}_{3}$ solution in combination with Sigma H5882 CTAB, perhaps we may have arrived at the same conclusion as Smith et al [21] and not decided to investigate the matter further. 


\section{Conclusions}

The most important conclusion is that iodide impurities can vary significantly from lot to lot within a CTAB product. It should not be a forgone conclusion that nanorods can or cannot be synthesized using certain $\mathrm{CTAB}$ products just based upon supplier name and product number; a lot number is required to make such judgements. Further, even with undetectable iodide impurities in $\mathrm{CTAB}$ products, variations in yields and dimensions of the nanorods produced across these products, points to possible influence of trace quantities of iodide or other impurities on the fundamental mechanism of nanorod formation. More research is required to identify contaminants and experimental conditions that can compromise reproducibility in gold nanorod synthesis.

\section{Acknowledgement}

The authors are grateful to Ms. C. Hermans (MiPlaza, Philips Research) for support during the analytical work. Dr. J. L. Hueso, Dr. D. K. Smith and Prof. B. A. Korgel (University of Texas at Austin) are thanked for their help in making their CTAB lot numbers available, and for sharing their experiences with lot-lot vagaries. The work is funded through the thrust area program NIMTIK of the University of Twente; through the PRESMITT project (IPD067771) of the SenterNovem program IOP Photonic Devices; and by the Nederlandse Wetenschappelijk Organisatie (NWO) and Stichting Technische Wetenschappen (STW) through project TTF 6527.

\section{References}

1) X. Huang, I.H. El-Sayed, W. Qian and M. A. El-Sayed, J. Am. Chem. Soc., vol. 128, pp. 2115-2120, 2006.

2) H. Liao, C.L. Nehl and J.H. Hafner, Nanomedicine, vol. 1, pp. 201-208, 2006.

3) X. Huang, P.K. Jain, I.H. El-Sayed, M. A. El-Sayed, Nanomed., vol. 2, pp. 681-693, 2007.

4) J. Pérez-Juste, I. Pastoriza-Santos, L.M. Liz-Marzán, P. Mulvaney,. Coord. Chem. Rev., vol. 249, pp. $1870-1901,2005$.

5) C. J. Murphy, T.K. Sau, A. M. Gole, C. J. Orendorff, J. Gao, L. Gou, S. E. Hunyadi and T. Li, J. Phys. Chem. B, vol. 109, pp 13857-13870, 2005.

6) Li, P.-C.; Wang, C.-R. C.; Shieh, D.-B.; Wei, C.-W.; C.-K. L.; Poe, C.;Jhan, S.; Ding, A.-A.; Wu, Y.-. Opt. Exp. 2008, 16, 18605-18615.

7) Yu, Y.-Y.; Chang, S.-S.; Lee, S.-S.; Wang, C.-R. C. J. Phys. Chem. B. 1997,101, 6661-6664.

8) N.R. Jana, L. Gearheart and C. J. Murphy, J. Phys. Chem. B, vol. 105, pp. 4065-4067, 2001.

9) B. Nikoobakht and M. A. El-Sayed, Langmuir, vol. 17, pp. 6368-6374, 2001.

10) B. Nikoobakht and M. A El-Sayed,. Chem. Mater., vol. 15, pp. 1957-1962, 2003.

11) B.D. Busbee, S.O. Obare and C. J Murphy,. Adv. Mater., vol. 15, pp. 414-416, 2003.

12) A. Gole and C. Murphy,. J. Chem. Mater., vol. 16, pp. 3633-3640, 2004.

13) X. Jiang, A. Brioude and M. Pileni,. Colloid. Surf. A, 277, pp. 201 - 206, 2006.

14) M. Liu and P. Guyot-Sionnest,. J. Phys. Chem. B, vol. 109, pp. 22192-200, 2005. 
15) M. Grzelczak, J. Pérez-Juste, P. Mulvaney, L. M. Liz-Marzán, Chem. Soc. Rev., vol. 37, pp. 1783 1791, 2008.

16) Y. Xia, Y. Xiong, B. Lim, S.E. Skrabalak, Angew. Chem. Intl. Ed., vol. 48, pp. 60-103, 2009.

17) M. Grzelczak, A. Sánchez-Iglesias, B. Rodríguez-González, R. Alvarez-Puebla, J. Pérez- Juste, L.M. Liz-Marzán, Adv. Func. Mater., vol. 18, pp. 3780-3786, 2008.

18) D.K. Smith and B.A. Korgel, Langmuir, vol. 24, pp. 644-649, 2008.

19) L. Gou, C.J. Murphy, Chem. Mater., vol. 17, pp. 3668-3672, 2005.

20) N.J. Durr, T. Larson, D.K. Smith, B.A. Korgel, K. Sokolov, A. Ben-Yakar, Nano Letters, vol. 7, pp. 941-945, 2007.

21) D.K. Smith, N.R. Miller, B.A. Korgel, Langmuir , vol. 25, pp. 9518-9524, 2009.

22) R.G. Rayavarapu, W. Petersen, C. Ungureanu, J. Post, T.G. Van Leeuwen, S. Manohar, Int. J. Biomed. Imaging, 29817, 2007.

23) B.T. Draine and P.J. Flatau, J. Opt. Soc. Am. A, vol. 11, pp. 1491-1499, 1994.

24) C. Ungureanu, R.G. Rayavarapu, S. Manohar, T.G. Van Leeuwen, J. Appl. Phys., vol. 105, pp. 102032-9, 2009.

25) M. Eghtedari, A.A. Oraevsky, J.A. Copland, N.A. Kotov, A. Conjusteau, M. Motamedi, Nano Lett., vol. 7, pp. 1914-1918, 2007. 


\section{Supporting Information}

\section{Size distributions of nanoparticles produced}

\subsection{Using Acros $22716 \mathrm{~V}$}

The Scanning Electron Microscopy (SEM) images of the particles produced using Acros 22716V are shown in Figures 2(b) and (c) in the manuscript. These are analyzed to ascertain size distributions.

\subsection{Using Sigma H5882}

Figures 3(b), (c) and (d) (SEM images) in the manuscript are analyzed to ascertain size distributions. Table 1 consolidates the sizes of particles encountered in the images.

\section{Reference}

1) D.K. Smith, N.R. Miller, and B.A. Korgel, Langmuir, 25, pp. 9518-9524, 2009.

Table 1: Size distributions for samples prepared using the two `suspect' CTAB products for different volumes of $\mathrm{AgNO}_{3}$ used in the growth solutions. All dimensions of len (length), width and dia (diameter) are in nm; vol (volume) in $\mu \mathrm{l}$; conc (concentration of nanorods) in NR/ml. Further, 'num' is number, 'NR' is nanorods and 'NS' is nanospheres.

\begin{tabular}{l|c|cccc|cc|c}
\hline $\begin{array}{l}\text { CTAB } \\
\text { product }\end{array}$ & $\begin{array}{c}\mathrm{AgNO}_{3} \\
\text { vol }\end{array}$ & $\begin{array}{c}\text { NR } \\
\text { length }\end{array}$ & $\begin{array}{c}\text { NR } \\
\text { width }\end{array}$ & $\begin{array}{c}\text { aspect } \\
\text { ratio }\end{array}$ & $\begin{array}{c}\text { num } \\
\text { of NR }\end{array}$ & $\begin{array}{c}\text { NS } \\
\text { size }\end{array}$ & $\begin{array}{c}\text { num } \\
\text { of NS }\end{array}$ & $\begin{array}{c}\text { conc } \\
\text { of NR }\end{array}$ \\
\hline Acros & 20 & $35.8 \pm 4.8$ & $12.6 \pm 1.9$ & $2.8 \pm 0.6$ & 30 & $38 \pm 6.8$ & 96 & $1 \times 10^{10}$ \\
$22716 \mathrm{~V}$ & 50 & $44 \pm 3.2$ & $22 \pm 1.7$ & $2.0 \pm 0.1$ & 250 & $43 \pm 17.6$ & 14 & $1.8 \times 10^{10}$ \\
& & & & & & & & \\
\hline Sigma & 20 & N.A. & N.A. & N.A. & N.A. & $25 \pm 3.2$ & 250 & N.A \\
H5882 & 50 & $33 \pm 3.2$ & $15 \pm 1.7$ & $2.1 \pm 0.2$ & 180 & $26 \pm 5.5$ & 83 & $1.6 \times 10^{10}$ \\
& 250 & $45 \pm 3.6$ & $12 \pm 2.2$ & $3.8 \pm 0.7$ & 250 & $29 \pm 5.4$ & 12 & $4 \times 10^{10}$ \\
\hline
\end{tabular}


Chapter 4

\title{
Maneuvering gold deposition to tailor the tips of gold nanorods by changing initial ascorbate-gold ion ratios in synthesis
}

\begin{abstract}
We report here a one-pot, one-shot approach to synthesize gold nanorods with tunable tip morphologies, by changing concentrations of ascorbic acid in a standard wet chemistry method. While the end products of the conventional method are hemispherically-capped cylindrical (nanorod) structures, in the modified method various subtly changed shapes from the nanorod are obtained. These shapes range from nanorod to dog-bone, to dumbbell and finally irregularly-shaped structures. This variety of shapes can be obtained by increasing just one constituent of the recipe, the initial ascorbic acid volumes used in growth solutions. The changes in shape and size of nanorod are manifested as changes in the position of longitudinal plasmon peaks from the far-visible to near infrared region (NIR) of the spectrum. We present a model which extends the concept of under-potential deposition of silver with fast bromide binding, where this passivation progressively targets different facets of growing gold seed, as the availability of AA ions is changed. Deposition of gold on the relatively exposed facets then results in the observed subtle deviations from the original nanorod shapes.
\end{abstract}

This chapter has been submitted for publication to ACS Nano as "R.G. Rayavarapu, T.G. van Leeuwen and S. Manohar". 


\section{Introduction}

Surface plasmon resonances of gold nanoparticles produce strongly enhanced optical interaction peaks making these particles suitable as contrast agents for optical or photoacoustic imaging [1]. Gold nanospheres have a single plasmon resonant optical interaction peak in the visible region of the spectrum. This peak does not shift considerably with changes in size or in refractive index of the embedding medium. Hence, the use of gold nanospheres is not useful for in vivo applications, as light penetration in tissue is poor in this spectral region due to high absorption by hemoglobin. With the introduction of an asymmetry, as is the case with rod-shaped particles, two plasmon-drived peaks are produced. Of all the gold nanoparticle shapes, rod-shaped gold nanoparticles are unique in their optical properties due to their two-plasmon peaks. The transverse plasmon (TP) peak is seen in the visible region due to the excitation of plasmons along the short axis of the nanorod, and a reddened longitudinal plasmon (LP) peak due to creation of plasmon along the long axis of the nanorod [2]. The LP peak of gold nanorods can be red-shifted by increasing the asymmetry in the form of the aspect ratio of the particle, and can be positioned in the NIR region. This wavelength region is characterized by high penetration of light into tissue since absorption by tissue chromophores is low. Such particles with strong absorption in this spectral region can be used as contrast agents or as photo-induced therapeutic agents in in vivo applications [3].

The most popular method for synthesizing gold nanorods with LP peaks tunable in the far-red and NIR spectral regions is the wet chemical method of Nikoobakht and El-Sayed [4], or one of its many variation [5]. Here, hexadecyltrimethylammonium bromide (CTAB) stabilized gold spheres catalyze reduction of gold salt when they are added to a growth solution comprising $\mathrm{CTAB}$, gold salt, silver nitrate $\left(\mathrm{AgNO}_{3}\right)$ and ascorbic acid (AA) $[4,5]$. Gold deposition takes place on the gold spheres, which function as nucleating centers, but because $\mathrm{CTAB}$ assisted by silver passivates certain facets of the seed particles, gold deposition occurs preferentially on the exposed tips to yield rod-shaped particles $[6,7,8]$.

The final shape and size of the nanoparticles are dependent on the nature of nucleating seed $[9,10,11,12]$ and upon several conditions in the growth phase. By increasing e.g. the concentrations of $\mathrm{AgNO}_{3}$ in the growth solutions, the most dramatic controlled change in size can be achieved, which causes the production of nanorods with progressively higher aspect ratios causing red-shifting of LP peaks $[4,13]$. 
Several other variables such as $\mathrm{pH}[14,15]$, and CTAB concentrations [16] have been changed to have an effect on yield or change the hemispherically capped gold nanorods into peanuts or nanowires that could be required for various applications. Huang et al [17] changed AA concentrations in the growth solution and were able to synthesize nanodog-bone structures.

Another approach has been to use prepared gold nanorods as seed with the addition of AA [18, 19], glutathione/cysteine [20] and iodide [21], downstream of the synthesis protocol. This achieved controlled overgrowth of gold to obtained shape tuning of the rods into a wide variety of shapes such as dog-bone, peanut, octohedra and dumbbell $[18,19]$.

We report here the fine-tuning of the tip shapes of gold nanorods, beyond dog-bone shapes as described by Huang et al [17] to dumbbell shapes, only by modulating AA concentrations in the growth solution in the seed-mediated nanorod synthesis method. We explain the mechanisms, which lead to the shapes obtained in a model based on identifying facets likely to be capped by silver bromide. These target facets depend upon the relative ratios of ascorbate to gold ions in the growth solution. We thereby identify the facets, which are left exposed to gold deposition and use this to explain the shapes obtained.

\section{Materials and Methods}

\section{Synthesis of Gold nanorods}

Hydrogen tetrachloroaurate $\left(\mathrm{HAuCl}_{4} \cdot 3 \mathrm{H}_{2} \mathrm{O}, 99.99 \%\right)$ was purchased from Acros Organics (Belgium), Hexadecylcetyltrimethylammonium bromide (CTAB, 98\%) was obtained from Fluka (52370) (The Netherlands), Silver nitrate $\left(\mathrm{AgNO}_{3}, \mathrm{Merck}\right.$, Germany), L-Ascorbic acid (Sigma, The Netherlands). All solutions were prepared using Milli-Q Gradient System water (Millipore, Quantum R).

Step 1: Gold nanorods were prepared by a seed-mediated growth method using the method of Gou and Murphy [5] with slight modifications. Seed solution was prepared by adding $0.6 \mathrm{ml}$ of ice cold $0.01 \mathrm{M} \mathrm{NaBH}_{4}$ to $9.75 \mathrm{ml}$ of $0.1 \mathrm{M} \mathrm{CTAB}$ mixed with $0.25 \mathrm{ml}$ of $0.01 \mathrm{M}$ gold salt. The solution was stirred for 2 minutes. The seed solution appeared brownish-yellow in color.

Step 2: In a typical experiment, six flasks containing identical growth mixtures were prepared by adding $9.5 \mathrm{ml}$ of $0.1 \mathrm{M} \mathrm{CTAB}$ to $0.5 \mathrm{ml}$ of $0.01 \mathrm{M} \mathrm{HAuCl}_{4} .3 \mathrm{H}_{2} \mathrm{O}, 70 \mu \mathrm{l}$ of 0.01 M silver nitrate solution and mixed gently to yield an orange colored solution. In the standard 
method [5], $55 \mu \mathrm{l}$ of $0.1 \mathrm{M} \mathrm{AA}$ is added to the growth solution. We used varying volumes $(45,55,100,150$ and $200 \mu \mathrm{l})$ of $0.1 \mathrm{M} \mathrm{AA}$ to realize ratios of AA to gold in the growth solutions namely $0.9: 1,1.1: 1,2: 1,3: 1$ and $4: 1$ respectively. The addition of $45 \mu 1$ of AA did not change the color of the growth mixture. However, in the case of all other growth mixtures with higher AA volumes, the orange solution turned colorless.

Step 3: Twelve $\mu 1$ of the seed solution from Step 1, were added to each of the five growth mixtures. These nanorod samples were aged for 24 hours. The samples were centrifuged twice at $10000 \mathrm{RCF}$ (relative centrifugal force) for 20 minutes and dispersed in $10 \mathrm{ml}$ Milli Q water.

\section{Nanoparticle characterization}

Scanning Electron Microscopy (Zeiss-1550 - SEM) and Transmission Electron Microscopy (CM 30 Philips - TEM) were used to analyze size distributions and shape variations in the samples respectively. Samples were prepared for electron microscopy by centrifuging nanorods at $10000 \mathrm{RCF}$ for $20 \mathrm{~min}$, twice, to remove excess surfactant. For analysis using SEM, the nanorod pellet was re-dispersed in a $0.5 \mathrm{ml}$ of Milli Q water, and $8 \mu \mathrm{l}$ of the suspension was air-dried on a silicon wafer for 2 hours, flushed gently with inert gas to prevent oxidation. Particle sizes were estimated using NI Vision module (Labview, National Instruments) on the digital SEM images with at least 250 particles considered in each sample. For TEM analysis, the nanorod pellet was air-dried on a carbon-coated grid for around 20 minutes.

Collimated transmission spectra of samples were measured using the Shimadzu PC3101 UV-Vis-NIR spectrophotometer. The spectra were used for estimating number densities as:

$$
N=\frac{\mu_{e x t}}{Q_{\text {ext }} \pi r^{2}}
$$

where, $\mu_{\text {ext }}$ is the extinction coefficient of the samples calculated from the transmission data using the Beer-Lambert law, $Q_{\text {ext }}$ is the extinction efficiency of a particle simulated using numerical methods such as the Discrete Dipole Approximation [22, 23] and ' $r$ ' is the radius of an equivalent sphere obtained from sizes ascertained from SEM images. 


\section{Results}

\section{Optical Spectroscopy}

The optical extinction spectra of the gold nanostructures synthesized by changing the volume of AA within the growth solution keeping all other parameters constant are shown in Fig. 1.

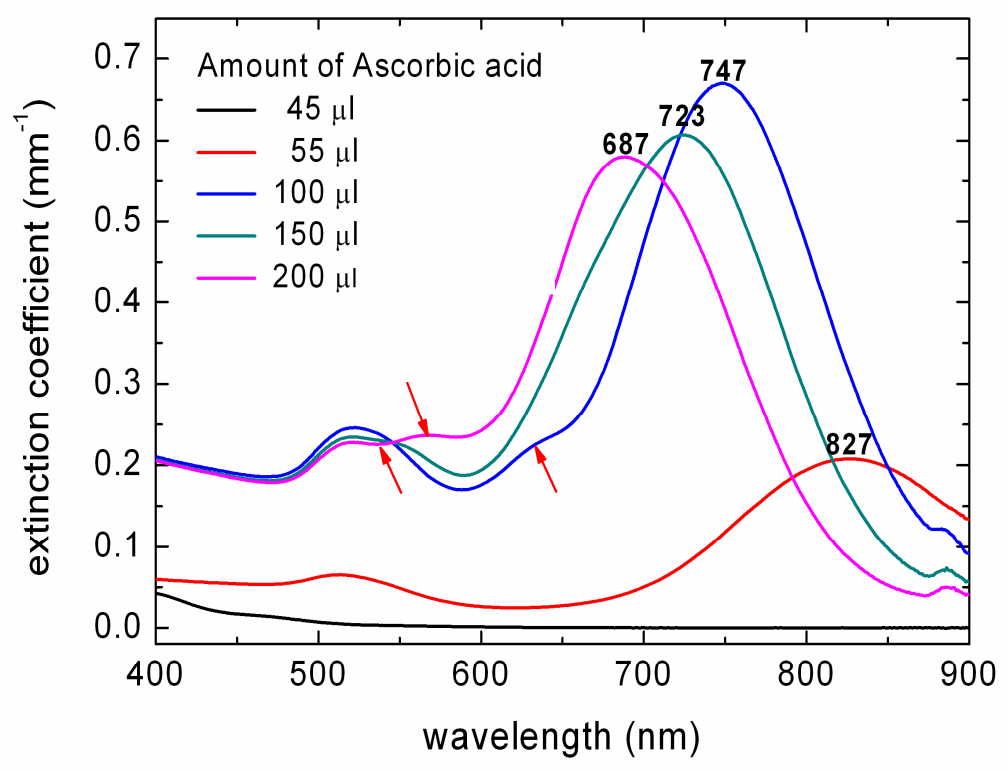

Figure 1: Optical extinction spectra of gold nanostructures synthesized with different volumes of ascorbic acid in the growth mixture. The particles produced have a progressively blue-shifted longitudinal plasmon peaks with increasing AA volumes. The arrows (red) mark shoulder peaks in the spectra of different gold nanostructures.

The absence of a LP peak with the case of $45 \mu \mathrm{lAA}$ in the growth solution shows that no gold nanorods are produced. When $55 \mu \mathrm{AA}$ is used in the growth solution, as in the original protocol of Ref. [5], gold nanorods are formed as observed in the characteristic TP and LP peaks at $513 \mathrm{~nm}$ and $827 \mathrm{~nm}$ respectively. From the LP peak, amplitude the concentration of particles is estimated to be $\sim 10^{10}$ particles $/ \mathrm{ml}$. With increasing volumes of AA (100, 150 and $200 \mu \mathrm{l})$ in the growth solutions nanostructures are formed, presumably nanorods, with progressive blue shifted LP peaks with higher amplitudes. In the samples with excess AA, shoulder peaks are observed in the optical spectra in the vicinity of 550, 575 and $625 \mathrm{~nm}$ respectively (marked with red arrows in figure 1) 


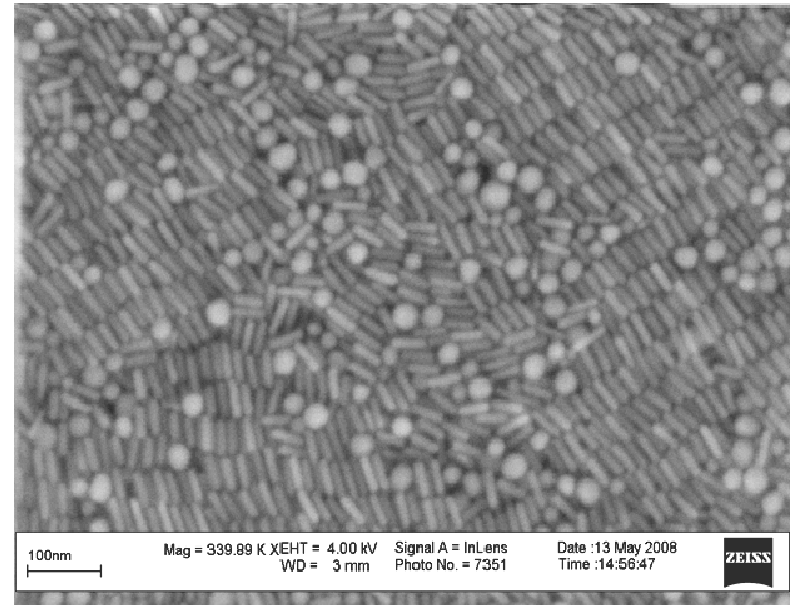

(a)

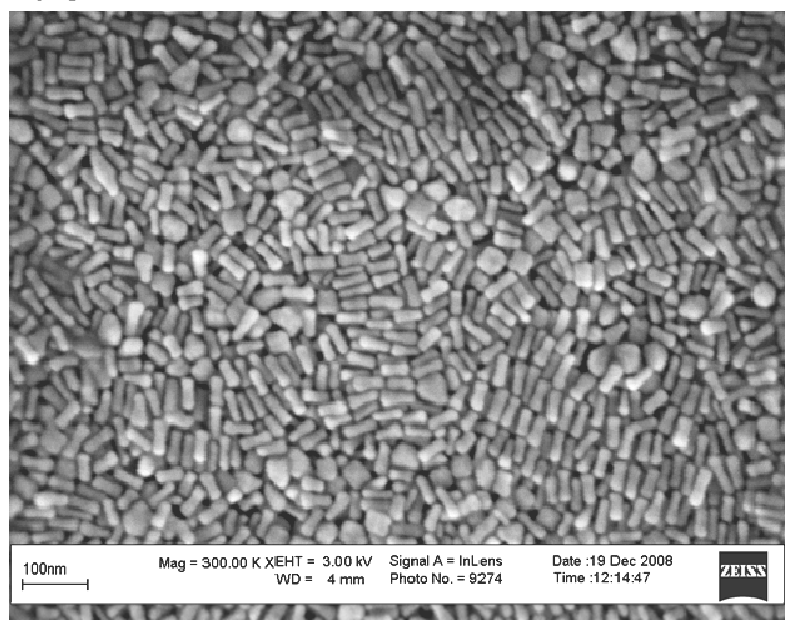

(c)

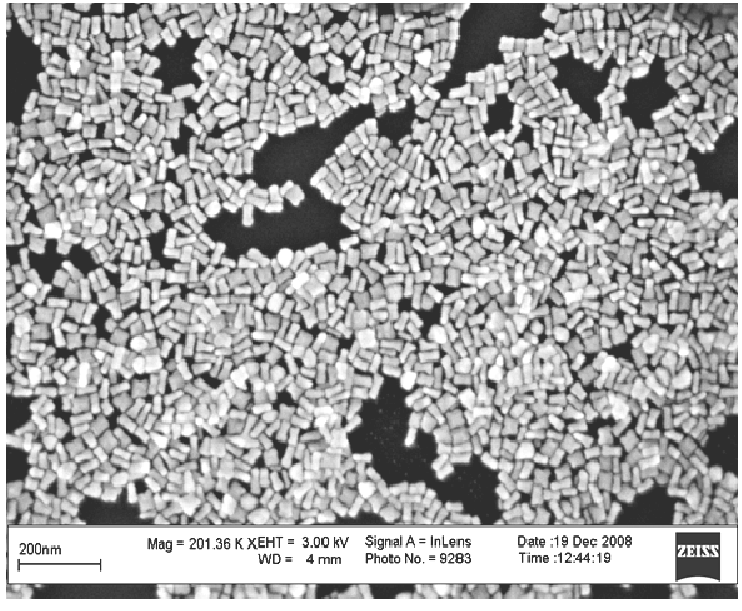

(b)

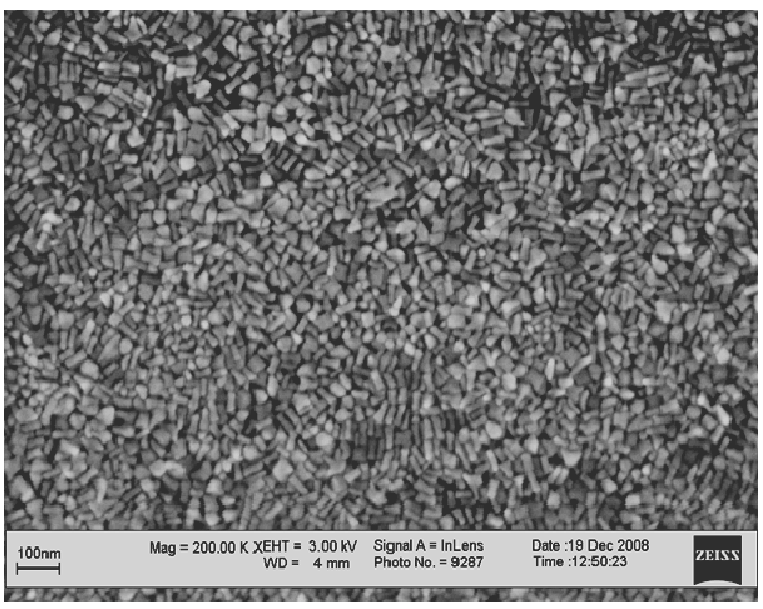

(d)

Figure 2: Scanning Electron Microscopic (SEM) images of various shapes of gold nanostructures synthesized with different AA:gold ion ratios in the growth mixtures. (a-d) Hemispherically-capped cylinder (nanorod) shaped, dog-bone shaped, dumbbell shaped and irregular shaped nanostructures are obtained when AA volumes used were 55, 100, 150 and $200 \mu 1$.

The nanostructures visualized using SEM, gives us preliminary information about the shape of nanostructures synthesized. The original protocol synthesizes hemispherically-capped cylinders (nanorods) as shown in figure 2(a) with a small amount of nanospheres as byproducts. With increase in the AA volumes in the growth mixtures, dog-bone, dumbbell and irregular shaped nanostructures are obtained as shown in figure 2(c)-(e). An overview of shapes obtained and the average sizes associated with these shapes determined from 250 particles in each SEM image (except for irregular shaped nanostructures) are shown in Table 1. Dog-bone shaped gold nanostructures are thicker compared to nanorod and dumbbell shaped nanostructures. The length of dumbbell is larger compared with that of dog-bone 
shaped nanostructures. All the nanostructures were measured in the waist region for their width.

$\begin{array}{ccccc}\text { AA volume }(\mu \mathbf{l}) & \text { Shape } & \text { Length }(\mathbf{n m}) & \text { Width }(\mathbf{n m}) & \text { Aspect Ratio } \\ 55 & \text { Nanorod } & 29.5 \pm 2.4 & 6.6 \pm 0.8 & 4.5 \pm 0.6 \\ 100 & \text { Dog-bone } & 42.0 \pm 4.8 & 13.8 \pm 2.3 & 3.1 \pm 0.6 \\ 150 & \text { Dumbbell } & 45.2 \pm 4.7 & 9.5 \pm 1.1 & 4.7 \pm 0.7 \\ 200 & \text { Irregular } & \text { NA } & \text { NA } & \text { NA }\end{array}$

Table 1: Shapes and average sizes obtained for the different cases of ascorbic acid volumes in the growth solution during nanorod synthesis. The mean length, width and aspect ratio are measured by taking 250 nanoparticles from each of the SEM images.

TEM analysis was done to visualize a single nanostructure to examine the details of the shape as shown in figure 3.

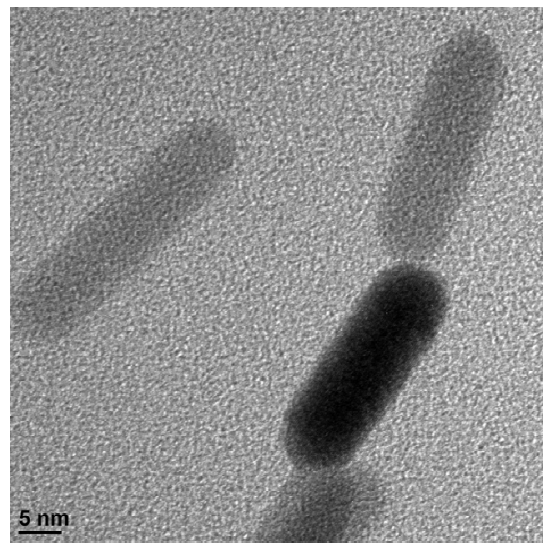

(a)

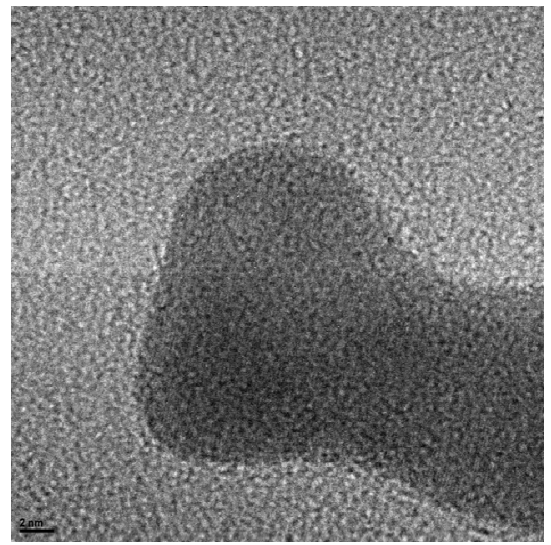

(c)

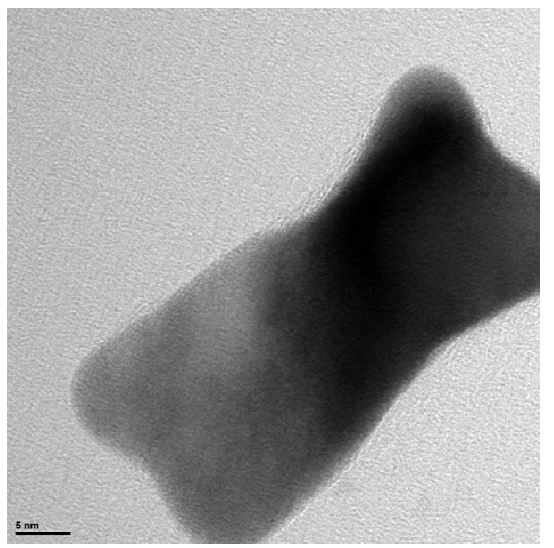

(b)

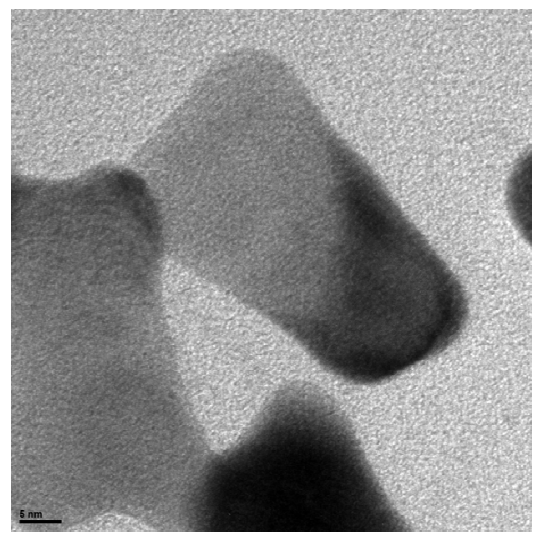

(d)

Figure 3: TEM image of the various shapes of gold nanostructures obtained when synthesis used different volumes of ascorbic acid in the growth solutions. (a) Hemispherically-capped cylinder (nanorod), (b) dog-bone shaped, (c) dumbbell shaped and (d) irregularly shaped nanostructures. Scale bar of images in (a), (b) and (d) is $5 \mathrm{~nm}$, and in (c) $2 \mathrm{~nm}$. 
When the standard method is used, the particles produced are the classic nanorod shape as shown in Figure 3(a). With the addition of $100 \mu \mathrm{l}$ of AA, the rod as it were, develops protrusions or lobes at the tips with thicker flanks to resemble a dog-bone shape as shown in Figure 3(b). The use of $150 \mu \mathrm{l} \mathrm{AA}$ in the growth mixture lead to the formation of a dumbbell shape where there is, as it were, a filling up at the tips of previous dog-bone shape as shown in Figure 3(c). The waist of the dumbbell is thinner than the previous structure. With $200 \mu 1$ AA in the growth solution, irregularly shaped nanostructures are produced as shown in Figure 3(d) where the growth appears not to have a preference in any one direction.

\section{Discussion}

Nanostructures are produced with morphologies subtly changed from the fundamental hemispherically-capped cylinder or nanorod shape with the use of increasing ascorbic acid (AA) to gold ratios in the growth solutions. The shapes progress from nanorod to dog-bone shaped to dumbbell shaped and finally into asymmetrically-flared indeterminate shapes as the starting $\mathrm{AA}: \mathrm{Au}^{3+}$ ratios are raised from the conventional 1.1:1 to an excess 4:1 as shown in Figure 4.

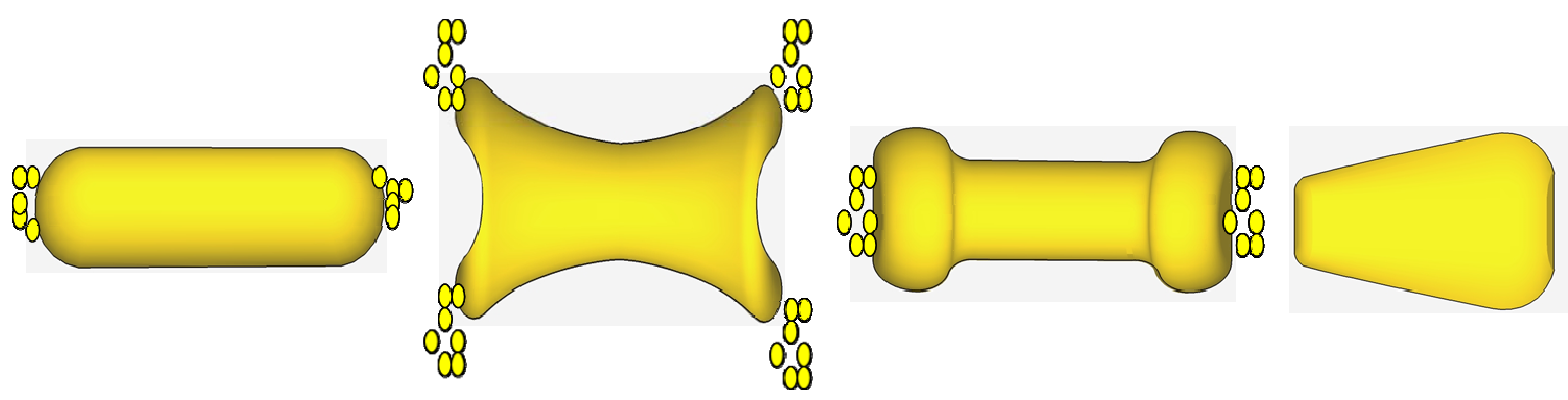

Figure 4: Schematic representation of gold nanostructures synthesized with increasing addition of AA during nanorod synthesis. The yellow dots represent gold atoms depositing on certain facets of Au.

Stoichiometric considerations allow us to roughly calculate the amount of gold atoms that can be reduced by the available ascorbate ions. The molar ratio of the ascorbate to gold to silver ions in the conventional silver-assisted synthesis method has been optimized to 1.1:1:0.14 [4, 5]. Ascorbate spontaneously reduces $\mathrm{Au}^{3+}$ to $\mathrm{Au}^{+}$in the first step thereby priming the growth solution to receive the gold seed. Addition of the CTAB-capped spheres catalyzes reduction of $\mathrm{Au}^{1+}$ to $\mathrm{Au}^{0}$ elemental gold. Each ascorbic acid molecule can supply two electrons $[8,18]$ while the change in oxidation state from $\mathrm{Au}^{3+}$ to $\mathrm{Au}^{0}$ requires 3 electrons. Further, $\mathrm{Ag}^{+}$ 
requires one electron for underpotential deposition (UPD), which plays a major role in symmetry breaking and nanorod growth as discussed further. In UPD, elemental silver is deposited on the growing surfaces of the gold seed even though ascorbic acid is not a strong enough reducing agent to change the oxidation state of $\mathrm{Ag}^{+}$at low $\mathrm{pH}$ [6]. Considering that UPD involves the formation of only a few monolayers of silver on the growing gold surfaces and that experimentally only $5 \%$ to $9 \%[7,19]$ silver has been found associated with the gold nanorod, practically all of the ascorbate is exhausted in reducing the gold salt. Supply and demand balances then allow one to estimate that up to $80 \%$ of the gold ions remain unreduced. For $100 \%$ utilization of the gold ions to form nanostructures a minimum AA: $\mathrm{Au}^{3+}$ ratio of 1.5:1 is required, which implies that for the ratios 2:1, 3:1 and 4:1 studied in this work, all gold ions have been exhausted in forming the particles.

With this background, we proceed to propose models to explain the formation of the observed shapes of synthesized nanostructures.

\section{Hemispherically-capped cylinder (nanorod) shape}

The formation of the nanorod shape has been discussed at length in the recent past. The model of Liu and Guyot-Sionnest [6] is able to satisfactorily explain many experimental observations, especially the exquisite control of nanorod aspect ratios by changing silver ion concentrations in the reaction mixtures. They proposed that in the process of underpotential deposition, the reduction of the $\mathrm{Ag}^{+}$to $\mathrm{Ag}^{0}$ occurs faster on $\{110\}$ facets of the CTAB stabilized gold seed crystal compared to the other facets as shown in figure 5.

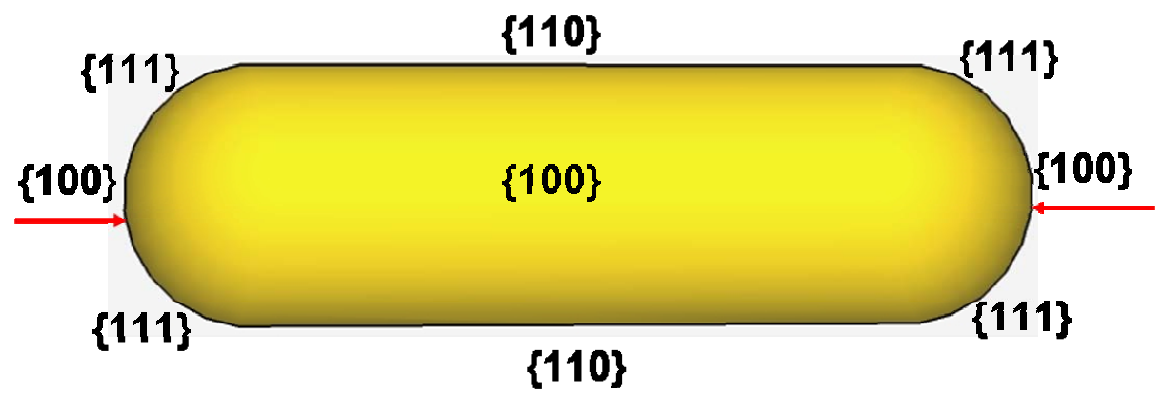

Figure 5: Schematic representation of cylindrical shaped gold nanorod with expected crystal facets [6]. The red arrows shows the location of the $\mathrm{Au}\{100\}$ at the tips.

Formation of the Ag-Br complex on this facet hinders the deposition of gold; symmetry breaking is initiated when the gold is reduced on the relatively exposed $\{100\}$ and $\{111\}$ facets to drive elongated nanorod growth predominantly in the [100] direction. The crystal 
facets observed in growing rods [6] shows dominant $\{110\}$ facets on the flanks with the $\{100\}$ as the terminating end facet; the $\{100\}$ facets on the flanks appearing possibly during aging.

\section{Dog-bone shape}

With an increased ascorbate to gold ion ratio to 2:1, all the gold ions can be reduced to elemental gold in nanostructures. Under the assumption that no new nucleation points are available with the use of the same seed solution as in the previous case, the one would assume a scaled up nanostructure with preservation of the hemispherical-capped cylinder shape. This has been observed by Ratto et al [18] with addition of ascorbic acid 48 hours after synthesis of nanorods, the addition phased over a period of 72 hours. In contrast to this slow secondary overgrowth on an existing template, our ascorbate excess being an intrinsic part of the initial growth solution has a strong influence on the shape determination. This is observed in structure that results, where the structure possesses lobes at either end to take on dog-bone morphology.

Since the changes in shapes are not radically different across the types made in this work, we believe that the rod-shaped structure (Figure 5) may be thought of as a basic model for the other shapes; the crystal growth facets will be the same for all. Insight into the shape evolution may be obtained by examining the crystal facet that is the target for the augmentation. While the terminating facets of the tips of the gold nanorod are $\mathrm{Au}\{100\}$, the rounded or angled parts of the tips are facets $\mathrm{Au}\{111\}$ and $\{110\}$ where gold deposition appears to take place.

To answer the question which of these facets will attract gold accretion and why we invoke the concept of silver UPD and bromide binding. As mentioned earlier, silver monolayers can be deposited on growing gold surfaces at potentials less negative than for bulk deposition [6] even with the low reduction potential of ascorbic acid at low $\mathrm{pH}$ values. The UPD shifts for the $\mathrm{Au}-\mathrm{Ag}^{+}$system follow the order $\mathrm{Au}\{110\}>\mathrm{Au}\{100\}>\mathrm{Au}\{111\}$ respectively $0.28 \mathrm{~V}, 0.17$ $\mathrm{V}$ and $0.12 \mathrm{~V}$. Silver deposition and subsequent bromide binding rates will be higher on the $\{110\}$ surface than the $\{100\}$ surface with the slowest passivation seen on the $\mathrm{Au}\{111\}$ edges. The gold deposition is thus directed predominantly to the $\{100\}$ and $\{111\}$ facet. At a certain point in time, the terminating facet $\{100\}$ is also capped by the silver bromide; gold deposition then proceeds practically solely on the stepped edge facets of $\mathrm{Au}\{111\}$ resulting in the observed protrusions. 
This is not seen in the conventional nanorod shape because the low ascorbate: $\mathrm{Au}^{3+}$ ratios ensure that the ascorbate ions are exhausted before gold augmentation of the $\{111\}$ facets can begin in earnest. This is also not seen when dosed ascorbic additions [18] are administered. This is perhaps because even though gold deposition would prefer the $\{111\}$ facets using the arguments above, the slow kinetics of gold deposition in overgrowth could allow migration of deposited adatoms into the $\{100\}$ face, a situation that has been observed in molecular dynamics simulation studies of gold nanorod growth [24]. It should be mentioned that Ratto et al [18] did indeed observe dog-bone like structures when fast secondary overgrowth is attempted with a single step addition of excess ascorbic acid, which can be explained using our arguments.

The increased extinction coefficient at the LP peak in the optical spectra (figure 1) is not due to a higher yield of particles since no new nucleating centers are introduced. With no extra seed to compete for gold, the accretion of the available gold takes place on the same number of particles causing the end products to be increased in volume (Table 1). With the extinction efficiencies maintaining roughly the same level as simulated by $\mathrm{Xu}$ and Cortie [25] using DDA methods, the observed increase in LP peak amplitude is a manifestation of the increased particle volume. The shoulder at $625 \mathrm{~nm}$ could be related to the loss of rotational symmetry as discussed earlier in Ref. 25 .

\section{Dumbbell shaped}

With a further increase in the ascorbate: gold ion ratio to $3: 1$, this shape is obtained also described as peanut shaped $[18,21,25,26]$. In this case, the number of gold ions that can be reduced remains the same as in the previous case, being $100 \%$. What is then the reason for the observed difference in shape with the end facet filled up? The difference in concentration of ascorbate ions may provide some hints here, since it may be expected that the availability of a higher number of such ions would lead to faster reaction dynamics. This will lead to a faster rate of silver UPD with bromide binding, capping the $\{110\}$ facets to impede gold deposition on the flanks more efficiently thereby pinching the structure strongly at the waist. More gold is then available for deposition at available facets and the deposition is steered early on to occur at the $\{100\}$ and $\{111\}$ facets with higher propensity for the latter. The otherwise sharp edges at the $\{111\}$ facets will see a rounding off as progressively more gold is deposited. 
Further the terminating facet could receive material by adatom migration from the excessively bulged $\{111\}$ facets as well as direct deposition from the growth solution. This would then lead to the observed structure that is a subtle change from the previous dog-bone shape.

The same arguments as used for the increased amplitudes of the LP peak in the dog-bone case are applicable here. However, while the aspect ratio (AR) of the particle is increased (Table 1) compared with dog-bone and nanorod cases, the LP peak is blue shifted. This may be explained in the following way. The $\mathrm{AR}$, as a simple ratio of maximum length to minimum width, is not to be used to predict the optical behavior of the particle. Examination of the shapes obtained (figures. 2(c) and 3(c)) reveals that the particle possesses relatively more matter along the transverse axis than is represented in the simple AR calculation. The observed blue shift in LP peak is thus not an anomaly since the effective AR is smaller than Table 1 suggests.

\section{Irregular shaped gold nanostructures}

As mentioned earlier, the relative preferences of "silver UPD with bromide binding" passivation of the facets largely follows the order $\mathrm{Au}\{110\}>A u\{100\}>A u\{111\}$, with gold deposition directed to follow an antipodal order of preference. When the ratio of ascorbate to gold ions in the growth solution is $4: 1$, a large excess of electrons is immediately available for driving the reduction of silver and gold salts. The kinetics of silver-bromide capping and the rates of gold deposition are ramped up. This then causes relatively uncontrolled gold deposition behavior where the competing growth at different facets is not as ordered or predictable as in the earlier shapes obtained. Even though the shapes are not well-defined, one does recognize semblances of the forms presented earlier showing that the basic mechanisms discussed earlier are still at play except that the behavior is less controlled.

\section{Conclusions}

We show that by increasing the volumes of ascorbic acid in growth solutions keeping the gold salt concentrations constant, during nanorod synthesis, various subtle changes in tip morphology from the conventional shape are obtained. The nanorod, dog-bone, dumbbell and finally indeterminate forms are seen as the ratios of ascorbate to gold ions are varied from the standard $1.1: 1$ to $2: 1,3: 1$ and $4: 1$. We are able to explain the shapes obtained in a model where passivation by silver-bromide progressively targets different facets of growing gold 
seed, the relatively exposed facets then being recipients of gold deposition. This mechanism is dictated by the rates of silver and gold deposition, which are increased due to the availability of excess ascorbate ions. We also explain the variations in observed optical responses due to the observed shapes.

\section{References}

1) J. Perez-Juste, I. Pastoriza-Santos, L. Liz-Marzan, P. Mulvaney, Coordination Chemistry Reviews., 249, pp. 1870-1901, 2005.

2) X. Huang, I.H. El-Sayed, and M.A. El-Sayed, J Am. Chem. Soc, 125, pp. 1215-1220, 2006.

3) I.H. El-Sayed, X. Huang and M.A. El-Sayed, Nano Lett, 5, pp. 829-834, 2005.

4) B. Nikoobakht and M.A. El-Sayed, Chem. Mater., 15, pp. 1957-1962, 2003.

5) L.F. Gou and C.J. Murphy, Chem. Mater., 17, pp. 3668-3672, 2005

6) M.Z. Liu and P. Guyot-Sionnest., J. Phys. Chem. B, 109, pp. 22192- 22200, 2005.

7) C.J. Orendorff, and C.J. Murphy, J. Phys. Chem. B., 110, pp. 3990-3994, 2006.

8) C.J. Murphy, T.K. Sau, A.M. Gole, C.J. Orendorff, J. Gao, L. Gou, S.E. Hunyadi and T. Li, J. Phys. Chem. B, 109, pp. 13857-13870, 2005.

9) N.R. Jana, L. Gearheart, and C.J. Murphy, J. Phys. Chem. B., 105, pp. 4065-4067, 2001.

10) N.R. Jana, L. Gearheart, and C.J. Murphy, Chem. Mater. vol. 13, pp. 2313-2322, 2001.

11) N.R. Jana, L. Gearheart and C.J. Murphy, Adv. Mater, 13, pp 1389-1393, 2001.

12) A. Gole, and C.J. Murphy, Chem. Mater., 16, pp. 3633 -3640, 2004.

13) R.G. Rayavarapu, W. Petersen, C. Ungureanu, J.N. Post, T.G. van Leeuwen and S. Manohar, IJBI, 29817, 2007.

14) K. Yong, Y. Sahoo, M.T. Swihart, P.M. Schneeberger and P.N. Prasad, Top Catal, 45, pp. 49-60, 2008.

15) W. Park, Y. Huh and W. Hong, Current Applied Physics., 9, pp. 140-143, 2009.

16) N. R. Jana, Small, 1, pp. $875-882,2005$.

17) C. Huang, P. Chiu, Y. Wang, T. Meen and C. Yang, Nanotechnology, 18, 395603, 2007.

18) F. Ratto, P. Matteini, F. Rossi and R. Pini, Journal Nanopart. Res. DOI 10.1007/s11051-009-9712-0, 2009.

19) M. Grzelczak, J. Perez-Juste, B. Rodriguez-Gonzalez and L.M. Liz-Marzan, J. Mater. Chem., 16, pp. 3946-3951, 2006.

20) C. Wang, T. Wang, Z. Ma and Z. Su, Nanotechnology, 16, pp. 2555-2560, 2005.

21) M. Grzelczak, A. Sánchez-Iglesias, B. Rodríguez-González, R. Alvarez-Puebla, J. Pérez-Juste, L. M. Liz-Marzán, Adv. Funct. Mater., 18, pp. 3780-3786, 2008.

22) B.T. Draine and P.J. Flatau, J. Opt. Soc. Am., pp. 1491-1499, 1994.

23) C. Ungureanu, R.G. Rayavarapu, S. Manohar, T.G. van Leeuwen, J. Appl. Phys., 105, 102032-9, 2009.

24) G. Grochola, I.K. Snook and S.P. Russo, J.Chem.Phys., 127, 2007.

25) X. Xu and M.B. Cortie, Adv. Funct. Mater., 16, pp. 2170-2176, 2006.

26) J.H. Song, F. Kim, D. Kim, and P. Yang, Chem. Eur. J., 11, pp. 910-916, 2005. 


\title{
Chapter 5
}

\section{In vitro toxicity studies of polymer-coated gold nanorods}

\begin{abstract}
We evaluated cellular responses to polymer-treated gold nanorods, which were synthesized using the standard wet-chemistry method that utilizes hexadecyltrimethylammonium bromide (CTAB). The nanorod dispersions were coated with either polystyrene sulfonate (PSS) or polyethylene glycol (PEG). Two sizes of nanorods were tested, with optical responses peaking at $628 \mathrm{~nm}$ and $773 \mathrm{~nm}$. The cells were from mammary adenocarcinoma (SKBR3), Chinese Hamster Ovary (CHO), mouse myoblast (C2C12) and Human Leukemia (HL60) cell-lines. Their mitochondrial function following exposure to the nanorods were assessed using the MTS-assay. We found PEGylated particles to have superior biocompatibility compared with PSS coated nanorods, which showed substantial cytotoxicity. Electron microscopy showed no cellular uptake of PEGylated particles compared with their PSS counterparts. PEGylated gold nanorods also exhibited better dispersion stability in the presence of cell growth medium; PSS-coated rods tended to flocculate or cluster. In the case of the PSS-particles, toxicity correlated with surface area across the two sizes of nanorods studied.
\end{abstract}

This chapter has been accepted for publication as "R.G. Rayavarapu, W. Petersen, L. Hartsuiker, P. Chin $\dagger$, H. Janssen†, F.W.B. van Leeuwen $\dagger$, C. Otto, S. Manohar and T.G. van Leeuwen”, Nanotechnology (in print). ( $\dagger$ - Netherlands Cancer Institute). 


\section{Introduction}

The interaction of light with gold nanoparticles has aroused much interest in the medical field, where these particles have potential as labels for light-based imaging techniques and as photo-induced therapeutic agents [1-4]. Light interaction with gold at the nanoscale is strong due to surface plasmon resonance, which leads to intense absorption or scattering peaks in the optical spectra [5]. Gold nanorods (AuNR) are characterized by two plasmon resonant peaks in the spectra. The lower energy peak, which arises from plasmons excited along the long axis of the rod, is called the longitudinal plasmon (LP) peak and can be tuned to occur in the far-red or near-infrared (NIR) between 600 - $950 \mathrm{~nm}$ [5]. The transverse plasmon (TP) peak due to plasmons excited along the AuNR's short axis remains pegged at around $520 \mathrm{~nm}$. The AuNRs' strong absorption or scattering can provide contrast for imaging using techniques such as photoacoustic imaging [6] and optical coherence tomography (OCT) [7]. Further, by optimizing the delivered light spatially and temporally, absorption by the particles can cause sufficient heating and localized temperature rise to kill cells, opening up therapeutic applications [8]. Nanorods (NRs) are ideal for applications in tissue, since light penetration is deep in the range of $700 \mathrm{~nm}-1100 \mathrm{~nm}$, as photons undergo less absorption and scattering by tissue components [9]. While much research has been done demonstrating the potential of these plasmonic nanoparticles in laboratory and pre-clinical studies, a crucial requirement for clinical translation is biocompatibility. With gold nanorods, an obstacle is the toxic surfactant hexadecyltrimethylammonium bromide (CTAB), which is indispensible for synthesizing particles with good yield, and size and shape control.

$\mathrm{CTAB}$ is used in growth solutions comprising gold salt, gold seed, silver nitrate and a weak reducing agent. Reduction of gold on the gold seed takes place, but because CTAB assisted by silver passivates certain facets of the seed particles, gold deposition occurs preferentially on the exposed tips to yield rod-shaped particles [10-12]. It has been shown earlier on a few cell-lines that repeated centrifugations and re-dispersion in water removes excess unbound $\mathrm{CTAB}$ and makes AuNR dispersions relatively less toxic [13-15]. However, centrifugation can strip stabilizing $\mathrm{CTAB}$ off the gold, which could result in aggregation of the particles. Further, it is possible that $\mathrm{CTAB}$ molecules can desorb from the gold surfaces, making such these particles potentially toxic. Thus, it is necessary to treat the particles in such a way either that the $\mathrm{CTAB}$ is removed from the particles or that it remains encapsulated. One approach to reduce the toxicity, has been to overcoat the $\mathrm{CTAB}$ particles with polyelectrolyte coatings such as poly(diallyldimethylammonium chloride) (PDADMAC), poly(4-styrenesulfonic acid) 
(PSS), polyacrylic acid (PAA) and poly(allylamine) hydrochloride (PAH), which prevents direct interactions between cells and CTAB, and shown to reduce cell-death in certain celllines $[16,17]$. Another approach is to PEGylate NRs, which has been shown to ameliorate cell toxicity in several cell lines $[13,14,15]$, by largely replacing CTAB.

We have expanded such cell-toxicity studies by applying PSS-coated and PEGylated nanorods to a number of cell lines. The cells used were SKBR3, CHO, C2C12 and HL60 cells. Cellular uptake in SKBR3 cells was also investigated using transmission electron microscopy (TEM). We studied two AuNR samples with plasmon peaks roughly occupying opposite ends of the optical imaging and therapeutic window $-628 \mathrm{~nm}$ and $773 \mathrm{~nm}$.

\section{Materials and Methods}

\section{Gold nanorod synthesis}

The following reagents were used: the gold salt (tetrachloroauric acid $\mathrm{HAuCl}_{4} \cdot 3 \mathrm{H}_{2} \mathrm{O}$ ) from Acros Organics (Belgium); hexadecyltrimethylammonium bromide (CTAB > 99\%), sodium borohydride $\left(\mathrm{NaBH}_{4}\right.$ 99\%) and ascorbic acid (99\%) from Fluka (The Netherlands); silver nitrate $\left(\mathrm{AgNO}_{3}\right.$ 99.8\%) from Merck (Germany). Prior to use, all glassware was cleaned with $8 \%$ hydrofluoric acid (HF), further with aqua regia $\left(\mathrm{HCl} / \mathrm{HNO}_{3}\right)$ and rinsed twice with Milli Q water.

AuNRs were synthesized using the seed-mediated method $[18,19]$ that requires the addition of gold seed to a growth solution with shape directing surfactant CTAB. The detailed protocol is described in Ref. [20], but we briefly describe the steps involved. Reducing agent $\mathrm{NaBH}_{4}$ is added to gold salt and surfactant CTAB. Within a few minutes as the reaction proceeds, the pre-formed spheres are used to seed a growth solution comprising gold salt, $\mathrm{CTAB}$, and $\mathrm{AgNO}_{3}$, which has been primed with the weak reducing agent ascorbic acid. Passivation of certain facets of the growing gold seed by CTAB assisted by silver $[10,11,12]$ causes symmetry breaking, and gold deposition is directed to occur on the exposed tips to yield rod-shaped particles. Using different $\mathrm{AgNO}_{3}$ concentrations in the growth solution allows a tuning of LP peak positions. Specifically, we used appropriate $\mathrm{AgNO}_{3}$ concentrations to synthesize two batches of NRs with LP peaks at 628 and $773 \mathrm{~nm}$ respectively. These are henceforth referred to as samples $A_{u N R} 628 \mathrm{~nm}$ and $A_{u N R} N_{73} \mathrm{~nm}$ respectively as shown in (Figure 1(a) and 2(a)).

The samples were filtered in two steps using a $5 \mu \mathrm{m}$ filter and a $0.45 \mu \mathrm{m}$ filter respectively to remove excess $\mathrm{CTAB}$ which tends to forms small crystals. This is followed by centrifugation 
at $10000 \mathrm{~g}$ for 20 minutes using a Beckman Coulter ultracentrifuge. The NR pellet from one centrifugation cycle, was dispersed in Milli Q water to give stock solutions of $100 \mathrm{ml}$ volumes with concentrations of the order of $10^{11} \mathrm{NRs} / \mathrm{ml}$.

\section{Polymer treatment of gold nanorods}

The following reagents were used: methoxy PEG-thiol (5000 MW) from Fluka (The Netherlands) and polystyrene sulfonate-Na salt (15000 MW) from PolySciences (USA).

\section{Polystyrene Sulfonate (PSS) treatment:}

To the NR stock solution $0.1 \mathrm{ml}$ of a PSS stock (10 mg PSS in $1 \mathrm{ml} 0.01 \mathrm{M} \mathrm{NaCl})$ and 0.05 $\mathrm{ml}$ of $0.01 \mathrm{M} \mathrm{NaCl}$ solution was added with gentle vortexing, and maintained at $4^{\circ} \mathrm{C}$ with stirring for 24 hours. The sample was then centrifuged and the supernatant with unbound PSS was discarded, and the pellet dispersed in $2.5 \mathrm{ml} 1 \mathrm{X}$ PBS.

\section{PEG-thiol treatment:}

The stock solution was centrifuged for a second time at $10000 \mathrm{G}$ for 20 minutes, and the NR pellet dispersed in $0.5 \mathrm{ml}$ of $5 \mathrm{mM}$ mPEG-SH with vigorous vortexing for 20 seconds. To this, $0.5 \mathrm{ml}$ of 1X PBS (Phosphate Buffer Saline) was added followed by continuous rotation for 24 hours at $4^{\circ} \mathrm{C}$. The resultant was subjected to two centrifugation steps to remove excess mPEG-SH from the solution and the pellet dispersed in $2.5 \mathrm{ml} 1 \mathrm{X}$ PBS [21].

\section{Nanorods Characterization}

Electron microscopy of samples (figure 1(a) and 2(a)) was performed using the Zeiss-1550 scanning electron microscope (SEM). Particle sizes were estimated using NI Vision module (Labview, National Instruments) on the digital SEM images with at least 250 particles considered in each sample. (See Table 1 for sizes of particles synthesized).

Collimated transmission spectra of samples were measured using the Shimadzu PC3101 UVVis-NIR spectrophotometer at various stages after synthesis and polymer treatment. Retention of the plasmon peaks in the spectra is taken as evidence of colloidal stability following processing steps. Further, the spectra are used for estimating number densities as:

$$
N=\frac{\mu_{\text {ext }}}{Q_{\text {ext }} \pi r^{2}}
$$

where, $\mu_{\text {ext }}$ is the extinction coefficient of the samples calculated from transmission data 
using the Beer-Lambert law (figure 1(a) and figure 2(a)), $Q_{\text {ext }}$ is the extinction efficiency of a particle simulated using numerical methods such as the Discrete Dipole Approximation [22, 23], and ' $r$ ' is the radius of an equivalent sphere obtained from sizes ascertained from SEM images. The concentration in number of NRs/litre is also expressed in a molar concentration in particle entities.

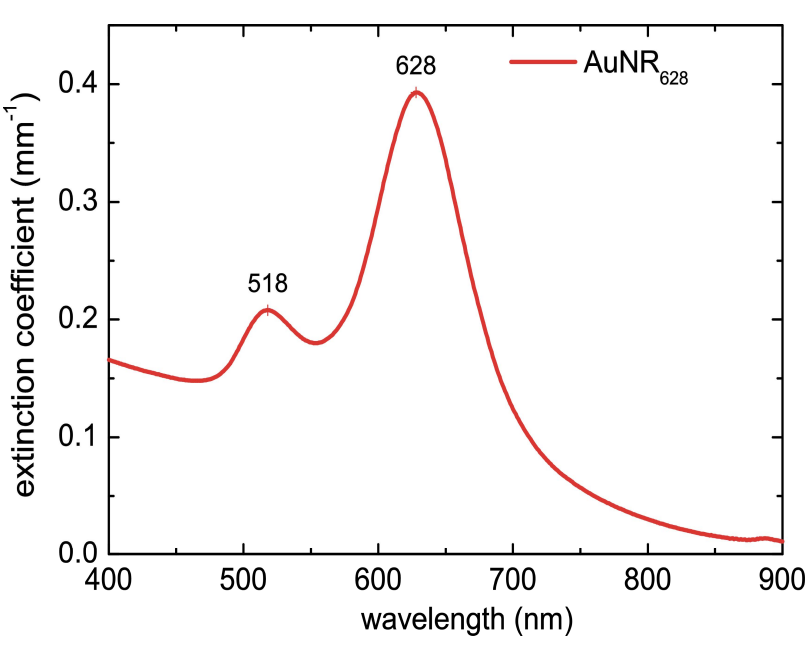

(a)

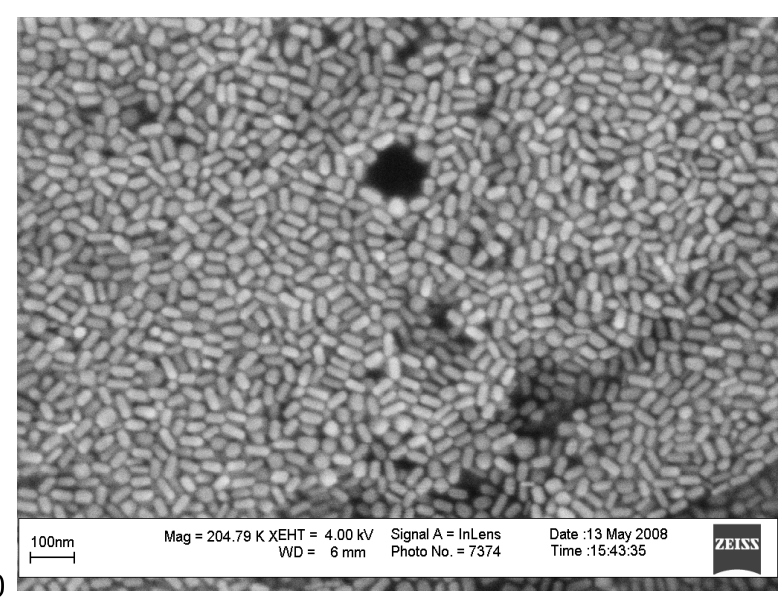

(b)

Figure 1: (a) Optical extinction spectrum of the batch of gold nanorods with longitudinal plasmon at $628 \mathrm{~nm}$. The batch is designated as AuNR 628 . (b) Scanning electron micrographs (SEM) of the same batch showing monodispersity of the particles.

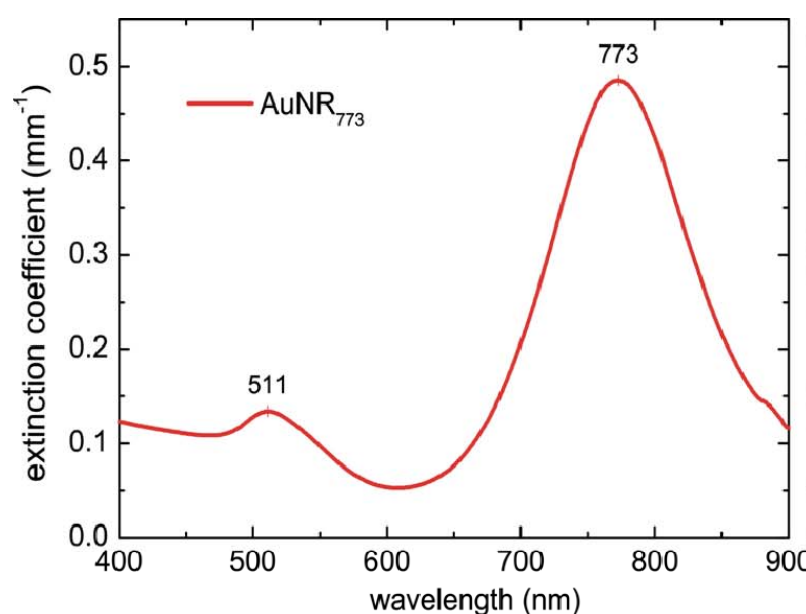

(a)

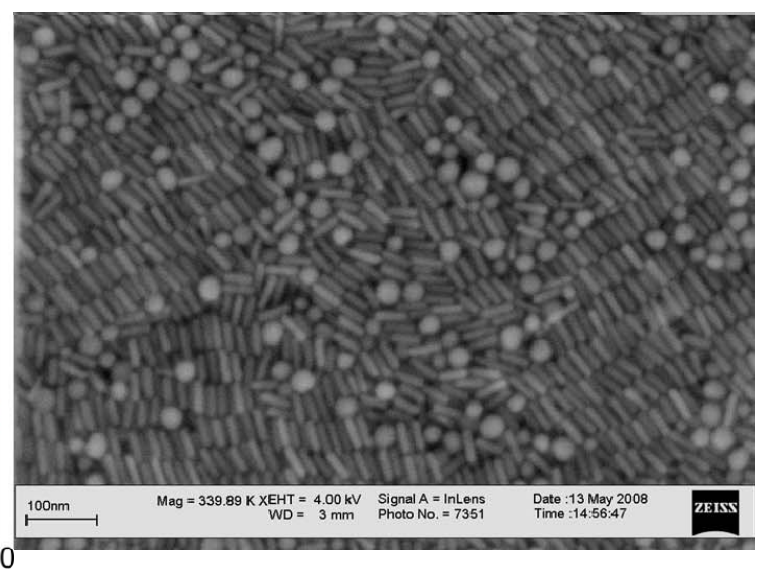

(b)

Figure 2: (a) Optical extinction spectrum of batch designated as $\mathrm{AuNR}_{773}$ showing the longitudinal plasmon peak at $773 \mathrm{~nm}$. (b) Scanning electron micrographs (SEM) of the same batch showing monodispersity of the particles. 


\begin{tabular}{lccc}
\hline Sample & $\begin{array}{c}\text { length } \\
(\mathrm{nm})\end{array}$ & $\begin{array}{c}\text { width } \\
(\mathrm{nm})\end{array}$ & $\begin{array}{c}\text { aspect } \\
\text { ratio }\end{array}$ \\
\hline AuNR $_{628}$ & $44.8 \pm 2.8$ & $18.5 \pm 1.6$ & $2.4 \pm 0.2$ \\
AuNR $_{773}$ & $41.8 \pm 3.3$ & $11.7 \pm 1.4$ & $3.5 \pm 0.4$ \\
\hline
\end{tabular}

Table 1: Average lengths, widths and aspect ratios of $A_{u N R} 628$ and $A_{u N R}$ s73 samples derived from analysis of at least 250 particles in each case from SEM images (Figures 1(b) and 2(b)).

The zeta- $(\zeta-)$ potential of Au-NRs was measured using the Malvern Zeta NS 2000 instrument (Malvern Instruments Ltd., UK). The zeta-potential serves as a criterion for inspecting surface charge changes on the nanoparticles, as well as a measure for their stability following various polymer-coating steps.

\section{Viability studies}

Adherent and suspension cells were studied. The former were mammary adenocarcinoma (SKBR3), Chinese Hamster Ovary (CHO) and mouse myoblast (C2C12) cells, whereas the suspension cells were Human Leukemia (HL60) cells. SKBR3 and HL60 cells were maintained using RPMI1640 medium supplemented with glutamine, 10\% FBS and 1\% antibiotics (penicillin/streptomycin). CHO and C2C12 were cultured using Dulbecco's Modified Eagle's Medium (DMEM) supplemented with glucose, 10\% FBS and 1\% antibiotics (penicillin/streptomycin). HL60 cells were passaged every 2 days whereas the SKBR3, CHO and C2C12 cells were passaged every 3 days.

Viabilities of the cells as a response to AuNR exposure, were studied using the CellTiter 96 Aqueous Cell Proliferation Assay from Promega (The Netherlands). Viable cells can be quantified by a colorimetric change to the tetrazolium compound - MTS and an electron coupling reagent phenazine methosulfate (PMS). The MTS salt is converted into formazan by metabolically active cells [10]. The quantity of formazan product is measured by the amount of $490 \mathrm{~nm}$ absorbance directly from 96-well plates and is directly proportional to the number of living cells. 


\section{Protocol followed}

The adherent cells were added to specific wells of the plate to obtain approximately 5000 cells/well. The plate was equilibrated for 16 hours at $37{ }^{\circ} \mathrm{C}$ in a humidified, $5 \% \mathrm{CO}_{2}$ atmosphere. HL60 cells were then dispensed into other wells to obtain approximately 5000 cells/well. The test compounds were as-prepared, filtered, PEGylated and PSS-treated $\mathrm{AuNR}_{628}$ and $\mathrm{AuNR}_{773}$ rods. Each type was added in volumes of $50 \mu \mathrm{l}$ in 8 serial dilutions (Table 2) in triplicate to the cell-line wells.

\begin{tabular}{lcccc}
\hline Samples & $\begin{array}{c}\text { AuNR } \\
(\mathrm{pM})\end{array}$ & $\begin{array}{c}\text { Filtered AuNR } \\
(\mathrm{pM})\end{array}$ & $\begin{array}{c}\text { PSS-AuNR } \\
(\mathrm{pM})\end{array}$ & $\begin{array}{c}\text { PEG-AuNR } \\
(\mathrm{pM})\end{array}$ \\
\hline AuNR $_{628}$ & $20-0.15$ & $20-0.15$ & $174-1.3$ & $165-1.2$ \\
AuNR $_{773}$ & $20-0.15$ & $20-0.15$ & $165-1.2$ & $157-1.2$ \\
\hline
\end{tabular}

Table 2: Start and end concentrations of nanorods in used in cell viability studies. Serial dilutions by a factor 2 are made to achieve 8 concentrations per type per batch of nanorods. The molar concentrations are in particles and not in gold atoms.

The same series of dilutions in duplicate of the test materials with $50 \mu$ medium were used without any cells as a sample background (SB) control. Culture medium (100 $\mu$ RPMI and DMEM) alone was used as a medium-only control $\left(\mathrm{LC}_{0}\right)$. The cells prepared for the assay were also used as cell-only control $\left(\mathrm{LC}_{100}\right)$. The plate with its well contents was maintained at $37^{\circ} \mathrm{C}$ in a humidified, $5 \% \mathrm{CO}_{2}$ atmosphere. Following 24 hours exposure to the various AuNR samples, $20 \mu \mathrm{l}$ of MTS/PMS solution was added to all the wells. The plates were wrapped in aluminum foil to prevent exposure to light, which could cause degradation of the MTS/PMS solution. The plates were incubated for a further 4 hours at $37^{\circ} \mathrm{C}$ in a humidified, 5\% $\mathrm{CO}_{2}$ atmosphere. The absorbance was then recorded at $490 \mathrm{~nm}$ using the Tecan plate reader.

\section{Data processing and analysis}

For every dilution, the average absorbance for the cells exposed in triplicate with test and assay compounds was calculated. This was corrected by deducting the average absorbance from the sample background control, and with a standard deviation calculated from a linear combination of errors [24]. As an example, 


$$
\left\langle A_{T C}\right\rangle_{\text {corr }}=\left\langle A_{T C}\right\rangle-\left\langle A_{S B}\right\rangle_{\text {and }} \sigma_{\text {corr }}=\sqrt{\sigma_{T C}^{2}+\sigma_{S B}^{2}}
$$

In the above, $A$ is the absorbance, $\sigma$ is the standard deviation with subscripts $T C$ and $S B$ representing test compound and sample background, respectively. Further, a corrected average $\mathrm{LC}_{100}$ absorbance is obtained by deducting average absorbance of $\mathrm{LC}_{0}$. This is taken as absorbance associated with living cells. The percentage cell survival is calculated as:

$$
\% \text { cell survival }=\left\lfloor\frac{\left\langle A_{T C}\right\rangle}{\left\langle\mathrm{LC}_{100}\right\rangle}\right\rfloor_{\text {corr }} \times 100 \%
$$

with a corresponding standard deviation calculated as the quotient combination of errors [24], which is the fractional errors of the individual components above:

$$
\sigma=\sqrt{\left(\frac{\sigma_{\text {corr }}}{\left\langle A_{T C}\right\rangle_{\text {corr }}}\right)^{2}+\left(\frac{\sigma_{\mathrm{LC}_{100}}}{\left\langle\mathrm{LC}_{100}\right\rangle}\right)^{2}} \times \% \text { cell survival }
$$

The cell survival percentages are plotted as a dose-response curve which permits the calculation of the $\mathrm{LC}_{50}$ value which is the $\mathrm{x}$-intercept of the curve at $50 \%$ cell death.

\section{Electron microscopy on gold nanorod exposed cells}

Due to the high x-ray attenuation of AuNRs, their interaction with cells can be accurately visualized at the nm-scale using Transmission Electron Microscopy (TEM). SKBR3 cells were exposed to the three variants of $\mathrm{AuNR}_{628}$ for $2 \frac{1}{2}$ hours. The concentrations used were 10 $\mathrm{pM}\left(6 \times 10^{9} \mathrm{NRs} / \mathrm{ml}\right)$ for PSS- and PEG-AuNR 628 , and $0.1 \mathrm{pM}\left(6 \times 10^{7} \mathrm{NRs} / \mathrm{ml}\right)$ for CTABAuNR $_{628}$. Exposed cells were fixed in Karnovsky fixative. Post-fixation was done with $1 \%$ osmium tetroxide in $0.1 \mathrm{M}$ cacodylate buffer.

After washing, the pellets were stained en bloc with Ultrastain-1 (Leica, Vienna, Austria), followed by an ethanol dehydration series. Finally, the cells were embedded in a mixture of DDSA/NMA/Embed-812 (EMS, Hatfield, USA), sectioned and stained with lead oxide and analyzed with a CM10 electron microscope (FEI, Eindhoven, the Netherlands). Only intact cells were analyzed. 


\section{Results}

\section{Characterization of polymer-treated gold nanorods}

Figures 3(a) and (b) are the optical extinction spectra of variously processed gold nanorods of the two batches $A_{u N R} 628$ and $A_{u N R}$ 773. The processing is either filtration to minimize excess CTAB, PSS-treatment or PEG-treatment. The spectra of the processed particles retain the LP peaks of the as-prepared NRs, with only marginal shifts in position, indicating that particle clustering or aggregation has not taken place. These spectra also remain stable in time as tested over several months. Peak amplitude differences (Figures 3(a) and (b)) are present for the various processing steps because concentrations were not normalized at the point when the spectra were measured.

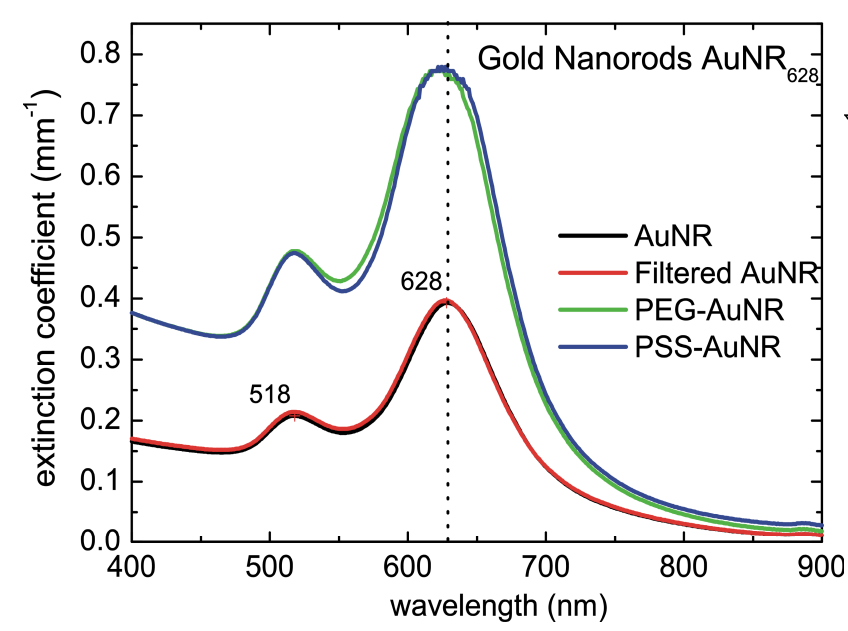

(a)

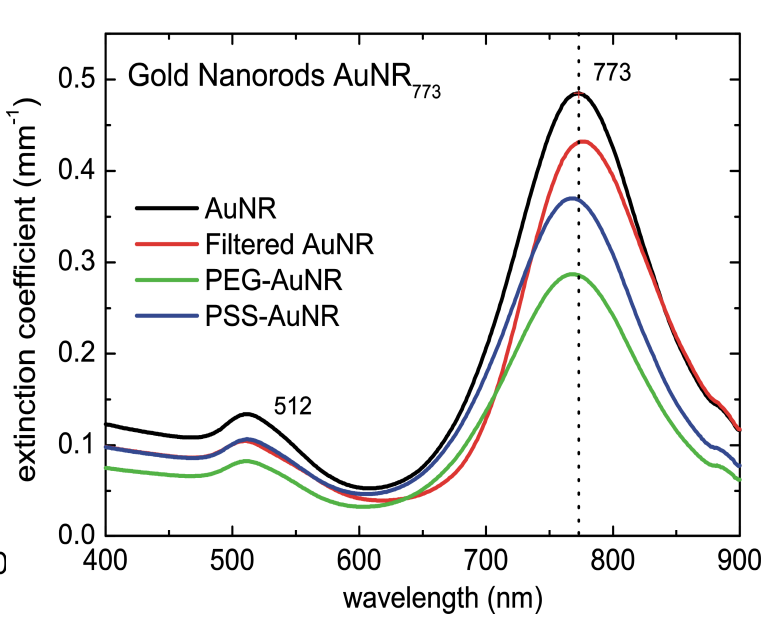

(b)

Figure 3: Optical extinction spectra of gold nanorods for the conditions of as-prepared, filtered, PSS-treated and PEGylated for batches designated as (a) $A_{u N R} 628$ and (b) $A_{u N R}$. The longitudinal plasmon peak features are retained indicating that polymer-treatment has not affected colloidal stability.

Table 3 shows the zeta-potential of various samples of the two NR types. The unprocessed and filtered AuNRs show high positive zeta-potential, which is consistent with the cationic head group $\left(\mathrm{CTA}^{+}\right)$of CTAB in the bilayer [25] on the gold surface. The PEG-AuNR show a low value of zeta-potential pointing to the presence of near-neutral PEG on the particle. In the case of PSS-AuNR, the presence of the anionic sulfonate groups of PSS is evident in the high negative values. 


\begin{tabular}{lcccc}
\hline Samples & $\begin{array}{c}\text { AuNR } \\
(\mathrm{mV})\end{array}$ & $\begin{array}{c}\text { Filtered AuNR } \\
(\mathrm{mV})\end{array}$ & $\begin{array}{c}\text { PSS-AuNR } \\
(\mathrm{mV})\end{array}$ & $\begin{array}{c}\text { PEG-AuNR } \\
(\mathrm{mV})\end{array}$ \\
\hline AuNR $_{628}$ & $+37.7 \pm 3.7$ & $+42.2 \pm 1.1$ & $-60.5 \pm 1.0$ & $-5.2 \pm 0.5$ \\
AuNR $_{773}$ & $+55.2 \pm 2.1$ & $+58.7 \pm 3.6$ & $-49.6 \pm 2.6$ & $-4.1 \pm 0.9$ \\
\hline
\end{tabular}

Table 3: Average zeta-potential values of typical as-prepared and variously treated gold nanorods for the two batches. Values are seen to be nearly neutral for PEG-treated samples and negative for PSS-treated nanorods.

The zeta-potentials of $\mathrm{AuNR}_{628}$ purified after incubation with the cell culture medium used are consolidated in Table 4.

\begin{tabular}{lccc}
\hline Samples & $\begin{array}{c}\text { AuNR }_{628} \\
(\mathrm{mV})\end{array}$ & $\begin{array}{c}\text { PSS-AuNR }_{628} \\
(\mathrm{mV})\end{array}$ & $\begin{array}{c}\text { PEG-AuNR }_{628} \\
(\mathrm{mV})\end{array}$ \\
\hline Before & $+53 \pm 7.2$ & $-45.0 \pm 3.6$ & $-4.1 \pm 0.6$ \\
After & $+5.2 \pm 2.1$ & $-7.0 \pm 4.6$ & $-0.3 \pm 0.6$ \\
\hline
\end{tabular}

Table 4: Average zeta-potential of typical gold nanorods $\left(\mathrm{AuNR}_{628}\right)$ before and after incubating with cell medium. Values are seen to drop to nearly zero from their initial values.

The original values fall to near neutral values for the CTAB-, and PSS-AuNR 628 showing compromised dispersion stability. The optical extinction spectrum of PSS-AuNR 628 after exposure to the growth medium (Figure 4) confirms clustering showing the appearance of a red-shifted broad peak.

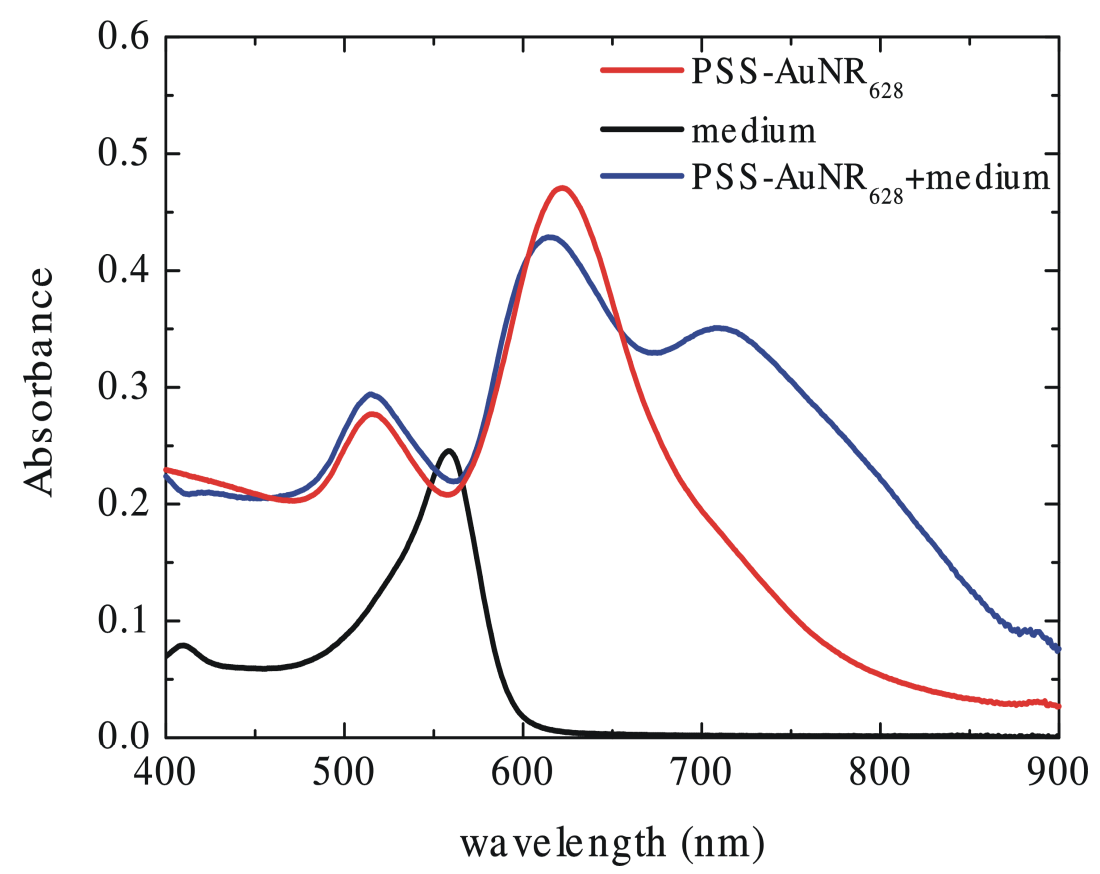

Figure 4: Optical extinction spectra of cell culture medium and of PSS-AuNR 628 before and after incubation in the medium. A reddened broad peak appears in the spectrum pointing to flocculation or clustering. 
The zeta-potentials of $\mathrm{AuNR}_{628}$ purified after incubation with the cell culture medium used are consolidated in Table 4. The original values fall to near neutral values for the CTAB-, and PSS-AuNR $_{628}$ showing compromised dispersion stability. The optical extinction spectrum of PSS-AuNR 628 after exposure to the growth medium (figure 4) confirms clustering showing the appearance of a red-shifted broad peak.

\section{Cell viability using gold nanorods}

Dose response curves using products from batch $\mathrm{AuNR}_{628}$ on cell-lines SKBR3, CHO, C2C12 and HL60 are shown in Figure 5(a)-(d) respectively.

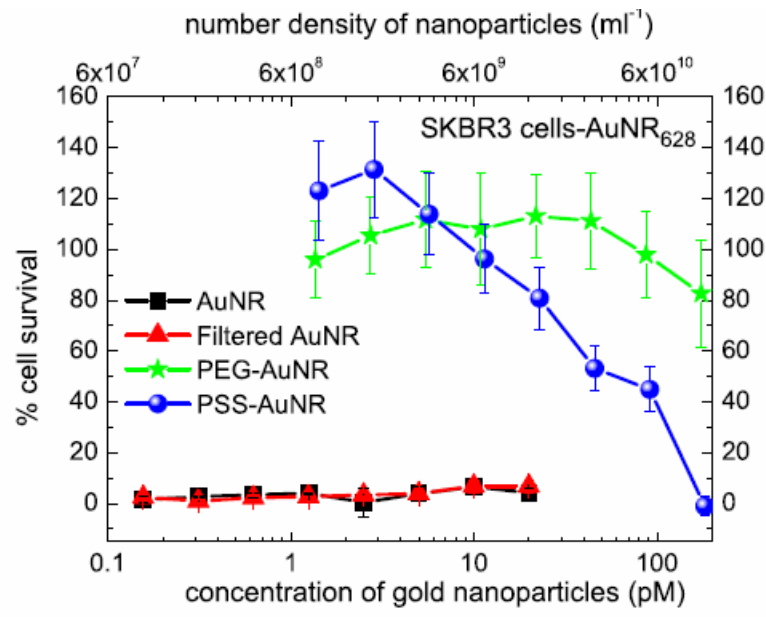

(a)

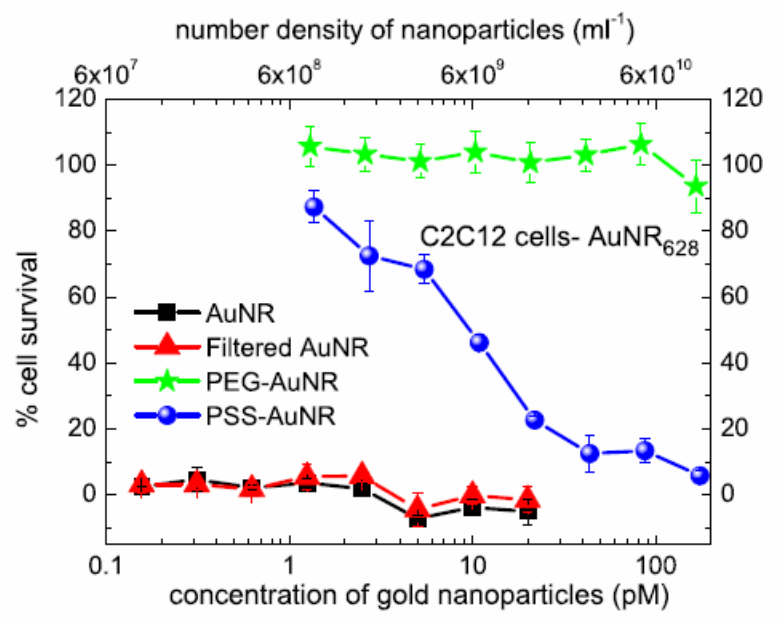

(c)

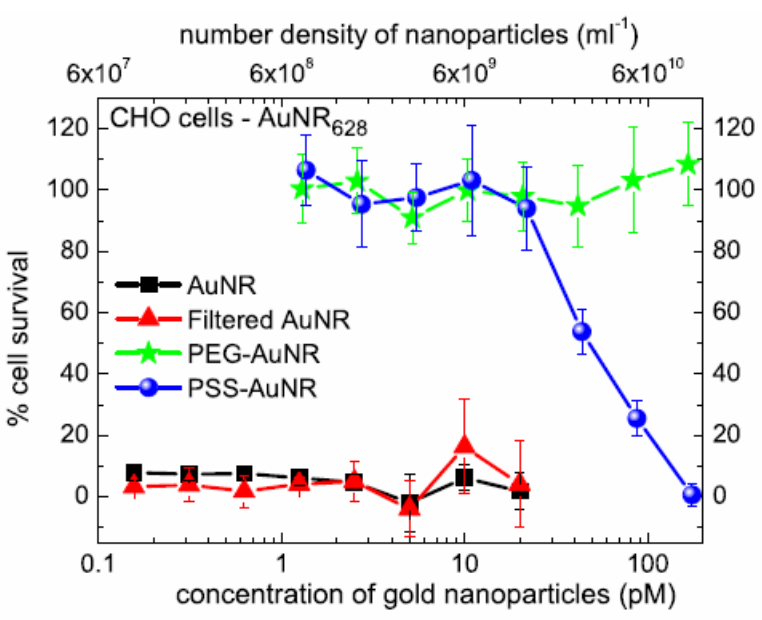

(b)

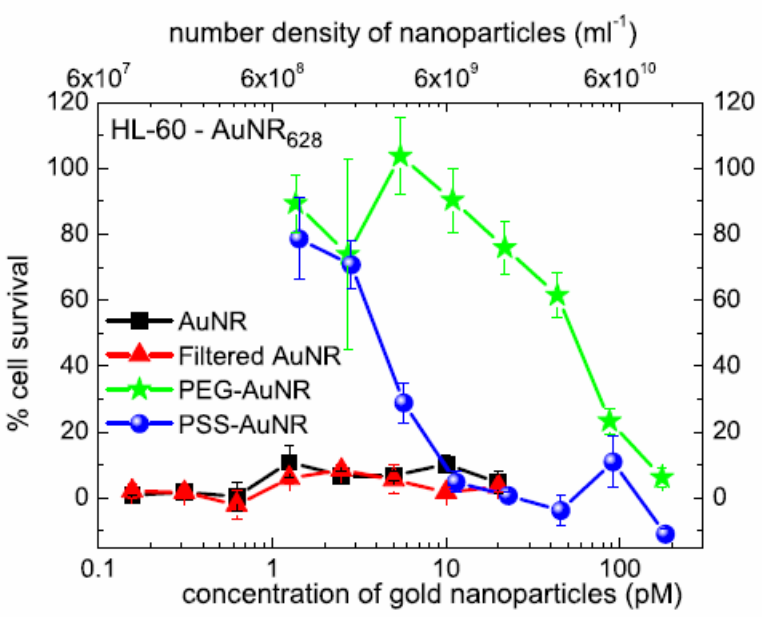

(d)

Figure 5: Dose-response curves of various cell-lines following exposure to gold nanorods (AuNR), filtered AuNR, PEGylated AuNR and PSS-treated AuNR. The results with batch AuNR 628 are shown with (a) SKBR3 cells, (b) $\mathrm{CHO}$ cells, (c) C2C12 cells, and (d) HL60 cells.

Data points and error bars have been calculated according to eqns. 4 and 5. For all cell-lines the as-prepared and filtered AuNR resulted in 100\% cell death even at the lowest 
concentrations. PSS-AuNR 628 gave high cell survival at low concentrations, which progressively worsened at higher concentrations for all cell-lines and particularly for HL60 cells. PEGylated nanorods evoked excellent cell survival for SKBR3, CHO and C2C12 cells for all concentrations. Only HL60 showed a toxic response at high concentrations of the PEGylated particles. The median lethal concentration $\mathrm{LC}_{50}$ values for exposure under the tested conditions were ascertained from the dose-response curves and are consolidated in Table 5.

\begin{tabular}{lcclr}
\hline Cell line & $\begin{array}{c}\text { AuNR } \\
\mathrm{pM}\left(\mathrm{ml}^{-1}\right)\end{array}$ & $\begin{array}{c}\text { Filtered AuNR } \\
\mathrm{pM}\left(\mathrm{ml}^{-1}\right)\end{array}$ & $\begin{array}{l}\text { PSS-AuNR } \\
\mathrm{pM}\left(\mathrm{ml}^{-1}\right)\end{array}$ & $\begin{array}{r}\text { PEG-AuNR } \\
\mathrm{pM}\left(\mathrm{ml}^{-1}\right)\end{array}$ \\
\hline SKBR3 & $<0.15\left(9 \times 10^{7}\right)$ & $<0.15\left(9 \times 10^{7}\right)$ & $28.4\left(1.7 \times 10^{10}\right)$ & $>165\left(1 \times 10^{11}\right)$ \\
CHO & $<0.15\left(9 \times 10^{7}\right)$ & $<0.15\left(9 \times 10^{7}\right)$ & $48.5\left(3 \times 10^{10}\right)$ & $>165\left(1 \times 10^{11}\right)$ \\
C2C12 & $<0.15\left(9 \times 10^{7}\right)$ & $<0.15\left(9 \times 10^{7}\right)$ & $9.7\left(6 \times 10^{9}\right)$ & $>165\left(1 \times 10^{11}\right)$ \\
HL60 & $<0.15\left(9 \times 10^{7}\right)$ & $<0.15\left(9 \times 10^{7}\right)$ & $3.0\left(1.8 \times 10^{9}\right)$ & $103\left(6 \times 10^{10}\right)$ \\
\hline
\end{tabular}

Table 5: The median lethal concentration $\mathrm{LC}_{50}$ values extracted from the dose-response curves of the tested cell-lines following exposure to gold nanorods (AuNR), filtered AuNR, PEG-AuNR and PSS-AuNR. All particles had their longitudinal plasmon peaks at $628 \mathrm{~nm}$.

In general $\mathrm{CHO}$ cells appeared to be less vulnerable to exposure to the different AuNRs compared with other cell-lines, while HL60 were most sensitive. The $\mathrm{LC}_{50}$ values for the PEG-AuNR 628 are indeterminate at the concentrations and exposures studied for adherent cells but are around $100 \mathrm{pM}$ corresponding to a number density of $6 \times 10^{10} \mathrm{NR} / \mathrm{ml}$ for the HL60 cells.

Similar trends were observed for the case of untreated and polymer treated- AuNR 773 . (See Supporting information, figure 1). The $\mathrm{LC}_{50}$ values for PSS-coated particles showed significant differences depending on whether $A_{u N R} 628$ or $A_{u N R}{ }_{773}$ were used; $A_{u N R} 628$ has lower $\mathrm{LC}_{50}$ values across all cell-lines showing that the cells have higher sensitivities to these particles. PEGylated particles $\mathrm{AuNR}_{628}$ and $\mathrm{AuNR}_{773}$ showed similar $\mathrm{LC}_{50}$ values on exposure to HL60 cells. (See Supporting Information, Table. 1).

\section{Electron microscopy of cellular uptake of gold nanorods}

In the case of the CTAB-coated AuNRs we found cell debris, indicating massive cell death, and could not detect AuNRs in any of the left over viable cells. The PSS-AuNR samples also 
displayed a high degree of cell death, but still enabled the detection of AuNRs in intact, but apoptotic cells. The PSS-AuNRs were located a vacuole together with cell debris and/or proteins (Figure 6).

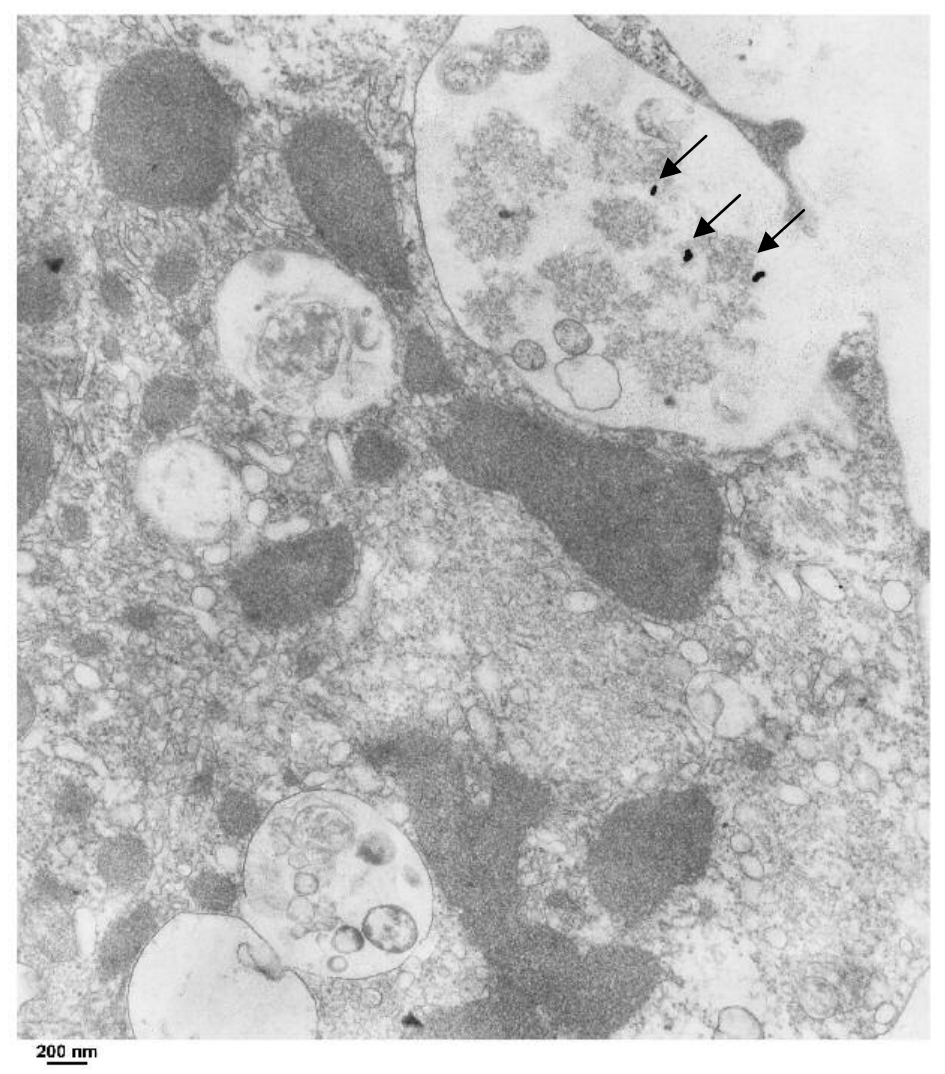

Figure 6: Transmission electron micrograph of an SKBR3 cells undergoing apoptosis after exposure to PSS$\mathrm{AuNR}_{628}$. Gold nanorods (marked with arrows) are seen in vacuoles.

Unfortunately, we were not able to determine the uptake route of these PSS-NRs. The cells incubated with the PEG-AuNRs, remained viable, however, no AuNRs were observed in these cells.

\section{Discussion}

It has previously been demonstrated on a few cell-lines that excess unbound CTAB makes AuNR dispersions toxic [13-17] by disrupting cell membrane integrity [26]. However, CTAB is crucial for the synthesis of AuNRs: CTAB (and silver) stabilize the $\{110\}$ faces of gold seed to a greater extent than the $\{100\}$ surfaces leading to a faster rate of gold atom growth on the latter, consequently leading to one-dimensional growth along the [100] direction [10, $11,12]$ to form rods. Further, the CTAB bilayer [25] imparts a net positive charge to the particle and helps preserve colloidal stability by electrostatic repulsion. Thus, CTAB is 
indispensable for low polydispersity, controllable aspect ratio $[18,27]$ and stability of the particles. Repeated centrifugation of AuNR can minimize free CTAB and thereby reduce toxicity. However, we found that a second cycle of centrifugation could in many cases result in irreversible aggregation of the particles. A single centrifugation following filtration gave stable AuNRs, but these were severely toxic even at the lowest concentrations of nanoparticles studied (figure 5).

\section{PSS-coated gold nanorods}

The model for PSS-treated particles is shown in figure 7(a). PSS being an anionic polyelectrolyte is electrostatically adsorbed on the positively charged CTAB bilayer. This forms an overcoat encapsulating CTAB-AuNRs. The zeta-potential values (Table 3) show a charge reversal and are evidence for this interaction. The magnitude of the zeta-potential, which is a measure of the repulsive forces present and an indicator of the potential colloidal stability, is greater than the rule-of-thumb $30 \mathrm{mV}$ [28]. This points to the stability of the colloidal system based on charge stabilization mechanisms, which is borne out from the largely unchanged plasmon peaks in figure 3(a) and (b), showing the absence of flocculation or coagulation.

Alkilany et al [17] showed that gold nanorods with certain polyelectrolyte surface coatings remained stable due to serum protein adsorption when incubated with cell growth medium. Our PSS-AuNRs on the other hand, suffered clustering when exposed to medium and FBS. This can be concluded from the optical spectra (figure 4), and from the zeta-potential values which drop to a few milliVolts $(\mathrm{mV})$ (Table 4). The presence of salts in the medium compromises charge stabilization due to electrostatic shielding. If there is sufficient protein adsorption on the particles, then the particles remain stable due to steric stabilization provided by the coating. In our case, at the concentrations chosen there appears to be inadequate adsorption of serum proteins making the AuNRs susceptible to flocculation. This has negative implications for use of such particles in vivo, since colloidal instability leads to loss of the predicted NR response due to two reasons. First, clustering weakens the LP peak intensity in the optical spectrum. Second, an initiation of an immune response to the agglomerates is also possible resulting in a loss of nanomaterial at target sites. When we exposed cells to low concentrations of PSS-AuNR 628 all cell-lines showed good viability $(80+\%)$. Cell-survival worsened with progressive increase in concentrations for three celllines; for HL60 cells the numbers dropped sharply to give a median $\mathrm{LC}_{50}$ an order of 
magnitude less than in the case of SKBR3 cells. The observed toxicity could be due to desorption of the PSS-CTAB complex [26] from the gold surface. This release of the toxic complex will be heightened when the particles cluster. The amount of the free complex increases with increasing concentrations and flocculation.

Why HL60 cells are more affected that others is discussed further. In the TEM images of cells exposed to PSS-AuNR 628 , a few clearly affected apoptotic, but still intact, SKBR3 cells can be found with NRs present inside vacuoles. These PSS-AuNRs appear to interact with cell debris and/or proteins. We believe that although the particles are susceptible to clustering, individual NRs or smaller clusters may still enter the cell via endocytosis. We intend to investigate aspects relating to cell-death, cellular uptake and passage in more detail.

\section{PEG-coated gold nanorods}

We incubated the particles with mPEG-thiol which results in strong sulphur binding to the gold surface with replacement of (most of) the CTAB bilayer. A model of the resultant is shown in figure 7(b). Evidence for this is provided in the zeta-potential values for the resultant (Table. 3) which are near-neutral indicating PEG replacement of most of the CTAB. There is no compromise in colloidal stability, since the PEG layer takes over the stabilizing role of $\mathrm{CTAB}$, but with a steric hindrance mechanism compared with electrostatic or charge stabilization observed with CTAB or with the PSS-AuNR. The optical spectra (figure 3(a) and (b)) of the particles before and after PEGylation show that the characteristic plasmon peaks are preserved and that the colloid is stable. The particles retain excellent dispersion stability even after mixing in cell culture medium showing that the PEG-SH forms a strong link to the gold surface, and the composite is not affected by cell medium contents. PEGylated particles elicited excellent cell viability and were essentially non-toxic for the SKBR3, $\mathrm{CHO}$ and $\mathrm{C} 2 \mathrm{C} 12$ cell-lines up to concentrations of $165 \mathrm{pM}$ corresponding to approximately $1 \times 10^{11} \mathrm{NRs} / \mathrm{ml}$. HL60 cells show a linear drop in cell-survival with increasing concentrations. Since neither nanogold nor PEG are themselves toxic, HL60 cell death could point to residual CTAB even after mPEG-SH treatment. However, this needs to be studied in detail. Absence of PEG-AuNR inside the intact SKBR3 cells observed using TEM suggests that PEG coatings prevent cellular uptake of the gold nanorods. In combination with the low toxicity profile (Figure 5) with the cell-line, this indicates that PEG-AuNRs have little interaction with SKBR3 cells. Huff et al [15] have also shown that cellular uptake of nanoparticles is reduced by their PEGylation. 


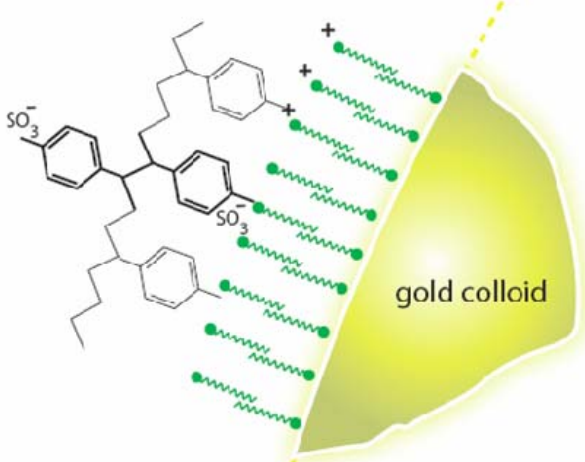

(a)

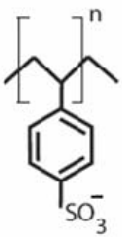

PSS

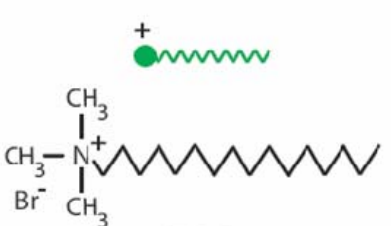

CTAB

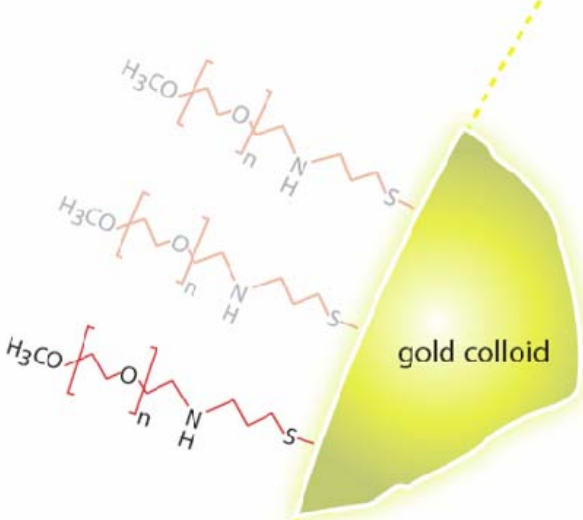

(b)

mPEG-SH

Figure 7: Models for surface modification of gold nanorods with (a) PSS, where an overcoat of the polymer occurs on the CTAB bilayer by electrostatic adsorption, (b) mPEG-SH, where sulphur-gold binding ensures replacement of the CTAB bilayer by PEG.

\section{Other remarks}

The surface area of single particle of $A_{u N R} 628$, calculated assuming that the NR is a hemispherically capped cylinder and using sizes from Table 1, is 1.69 times greater than for AuNR $_{773}$. The loading of the toxic CTAB bilayer is thus proportionally higher for PSS$\mathrm{AuNR}_{628}$ giving it a corresponding higher toxic potential than its $773 \mathrm{~nm}$ counterpart. This is the reason for the observed higher sensitivity of all cell-lines for exposure to PSS-AuNR 628 as assessed by $\mathrm{LC}_{50}$ values, compared to PSS-AuNR 773 (Table 6).

\begin{tabular}{lcccc}
\hline Samples & SKBR3 & CHO & C2C12 & HL60 \\
\hline PSS-AuNR $_{628}$ & $28.4 \mathrm{pM}$ & $48.5 \mathrm{pM}$ & $10 \mathrm{pM}$ & $3 \mathrm{pM}$ \\
PSS-AuNR $_{773}$ & $38 \mathrm{pM}$ & $57 \mathrm{pM}$ & $11 \mathrm{pM}$ & $4 \mathrm{pM}$ \\
\hline
\end{tabular}

Table 6: $\mathrm{LC}_{50}$ values extracted from Fig. 5 and Supporting information Fig. 1, showing differences in sensitivity of the cell-lines to PSS-coated gold nanorods of two sizes.

In the case of PEGylated particles, surface area is not the parameter of interest for comparing cytotoxicity between the two families of NRs, since the treatment largely replaces CTAB. 
Indeed for the case where $\mathrm{LC}_{50}$ values of PEG-AuNRs are determinate, namely for HL60 cells, there was no difference in the values. Different cells show different responses based on inherent characteristics such as surface reactivity, making a comparison across cell-lines complicated. The case of HL60 cells which show higher cell deaths compared with other celllines, may be an example of such natural characteristics, making this a less robust cell-line than the others. A part of the reason may also be due to HL60 being cells in suspension. The cells expose larger surface areas compared with the two-dimensional distribution of adherent cells, with a corresponding higher probability of interaction with toxic components in the microenvironment. While we have established that the biocompatibility of PEG-AuNR is superior to PSS-AuNR as manifested in the $\mathrm{LC}_{50}$ values, the question is whether such concentrations will ever be encountered in vivo at non-target sites to cause unwanted toxicity. This requires dosage studies in living organisms, where bioaccumulation of particles in organs [13] will need to be ascertained. In any case, we can place the $\mathrm{LC}_{50}$ concentrations in context, by calculating the NR load per cell that would be required for detection using photoacoustic imaging. We have estimated that a cell-NR ratio of $\sim 30$ marks minimum detectability using photoacoustics [29, 30]. (See Supporting Information for details.) Compare this with the cell-NR ratio of 360,000 calculated at the $\mathrm{LC}_{50}$ for PSS-AuNR 628 with HL60 cells.

\section{Conclusions}

The implication of this study on utilization is that PEGylated AuNRs are superior to PSScoated AuNRs from the point of view of biocompatibility. Treating the particles with mPEG$\mathrm{SH}$, replaces toxic $\mathrm{CTAB}$ with $\mathrm{PEG}$, and renders the particles colloidally stable even in the presence of salts in cell culture medium. Treatment with PSS and possibly other polyelectrolytes, is an inferior detoxification approach since the polymer only encapsulates the AuNR with its CTAB bilayer intact. PSS-AuNRs cluster and coagulate on exposure to cell culture medium, which will further hamper future use in vivo. This in vitro study is valuable in learning about necessary modifications to AuNRs in a quick and relatively inexpensive way to improve biocompatibility. Ultimately, we intend to test these particles in vivo with all the complexity of blood flow, immunological and inflammatory responses, and the biological variability inherent to such systems [31]. Another direction which is being explored is to use PEG not only to detoxify and stabilize the AuNRs, but also as a linker to attach appropriate antibodies at their free ends. 


\section{Acknowledgements}

This work is funded through the thrust area program NIMTIK of the University of Twente; through the PRESMITT project (IPD067771) of the SenterNovem program IOP Photonic Devices; and by the Nederlandse Wetenschappelijk Organisatie (NWO) and Stichting Technische Wetenschappen (STW) through project TTF 6527; and by a KWF-translational research award (Grant No. PGF 2009-4344; FvL).

\section{References}

1) J.L. West and N.J. Halas, Ann. Rev. Biomed. Engg., 5, pp. 285-292, 2003.

2) H. Liao, C.L. Nehl and J.H. Hafner, Nanomedicine, 1, pp. 201-208, 2006.

3) C.J. Murphy, T.K. Sau, A.M. Gole, C.J. Orendorff, J. Gao, L. Gou, S.E. Hunyadi and T. Li, J. Phys. Chem. B, 109, pp. 13857-13870, 2005.

4) X. Huang, I.H. El-Sayed, and M.A. El-Sayed, J Am. Chem. Soc., 125, pp. 1215-1220, 2006.

5) S. Eustis and M.A. El-Sayed, 35, pp. 209-217, 2006.

6) M. Eghtedari, A.A. Oraevsky, J.A. Copland, N.A. Kotov, A. Conjusteau and M. Motamedi, Nano Lett., 7, pp. 1914-1918, 2007.

7) A.L. Oldenburg, M.N. Hansen, T.S. Ralston, A. Wei and S.A. Boppart, J. Mater. Chem., 19, pp. 64076411, 2009.

8) X. Huang, P.K. Jain, I.H. El-Sayed and M.A. El-Sayed, Nanomed., 2, pp. 681-693, 2007.

9) B.J. Tromberg, N. Shah, R. Lanning, A. Cerussi, J. Espinoza, T. Pham, L. Svaasand and J. Butler, Neoplasia, 2, pp. 26-40, 2000.

10) M.Z. Liu and P. Guyot-Sionnest, J. Phys. Chem. B, 109, pp. 22192-22200, 2005.

11) J.C. Orendroff, and C.J. Murphy, J. Phys. Chem. B., 110, pp. 3990-3994, 2006.

12) M. Grzelczak, J. Prez-Juste, P. Mulvaney and L.M. Liz-Marzan, Chem. Soc. Rev., 37, pp.1783-1791, 2008.

13) T. Nidome, M. Yamagata, Y. Okamoto, Y. Akiyama, H. Takahashi, T. Kawano, Y. Katayama and Y. Niidome, J. Contl. Release, 114, pp. 343-347, 2006.

14) T.B. Huff, M.N. Hansen, Y. Zhao, J.X. Cheng and A. Wei, Langmuir, 23, pp. 1596-1599, 2007.

15) T.S. Hauck, A.A. Ghazani and W.C.W. Chan, Small, 4, pp. 153-159, 2008.

16) A.M. Alkilany, P.K. Nagaria, C.R. Hexel, T.J. Shaw, C.J. Murphy and M.D. Wyatt, Small , 5, pp. 701708, 2009.

17) B. Nikoobakht and M.A. El-Sayed, Chem. Mater, 15, pp. 1957-1962, 2003.

18) A.V. Alekseeva, V.A. Bogatyrev, L.A. Dykman, B.N. Khlebtsov, L.A. Trachuk, A.G. Melnikov and N.G. Khlebtsov, Appl. Opt., 44, pp. 6285-6295, 2005.

19) R.G. Rayavarapu, W. Petersen, C. Ungureanu, J. Post, T.G. van Leeuwen and S. Manohar, Int. J. Biomed. Imaging, 29817, 2007.

20) E.E. Connor, J. Mwamuka, A. Gole, C.J. Murphy and M.D. Wyatt, Small 1, pp. 325-327, 2005.

21) B.T. Draine and P.J. Flatau, J. Opt. Soc. Am. A., 11, 1994.

22) C. Ungureanu, R.G. Rayavarapu, S. Manohar and T.G. van Leeuwen, J. Appl. Phys. 105, 102032, 2009.

23) L. Lyons A practical guide to data analysis for physical science students Cambridge University Press, New York, 1991.

24) B. Nikoobakht and M.A. El-Sayed, Langmuir, 17, pp. 6368-6374, 2001.

25) A.P. Leonov, J. Zheng, J.D. Clogston, S.T. Stern, A.K. Patri and A. Wei, ACS Nano, 2, pp. 2481-2488, 2008.

26) J. Gao, C.M. Bender and C.J. Murphy, Langmuir, 19, pp. 9065-9070, 2003.

27) Malvern Instruments Ltd., Malvern Zetasizer Manual, 2008.

28) J. Jose, R. Willemink, S. Resink, T. Maalderink, J.C.G. van Hespen, T.G. van Leeuwen and S. Manohar 7371, 73710S, SPIE Proceedings, 2009.

29) J. Jose, T. Maalderink, G.H. Willemink, J.W. Van Neck, S. Manohar and T.G. van Leeuwen, $A$ Computed Tomography photoacoustic imager for imaging murine disease models, (in prep).

30) B.J. Panessa-Warren, J.B. Warren, M.M. Maye and W. Schiffer, Nanoparticles and Nanodevices in Biological Applications: The INFN Lectures, pp.1-46, 2009. 


\section{Supporting Information}

\section{Cell viability on exposure to $\mathrm{AuNR}_{773}$}

Dose response curves of cell-lines SKBR3, CHO, C2C12 and HL60 exposed to AuNR 773 are shown in Supporting Information Figure 1. Data points and error bars are calculated according to manuscript eqns. 4 and 5. Similar trends as in the case of exposure to AuNR 628 are seen. For all cell-lines the as-prepared and filtered AuNRs resulted in 100\% cell death even at the lowest concentrations. PSS-AuNR 773 gave high cell survival at low concentrations, which progressively worsened at higher concentrations for all cell-lines and particularly for HL60 cells. PEG-AuNR ${ }_{773}$ shows excellent cell survival for all but HL60 cells.

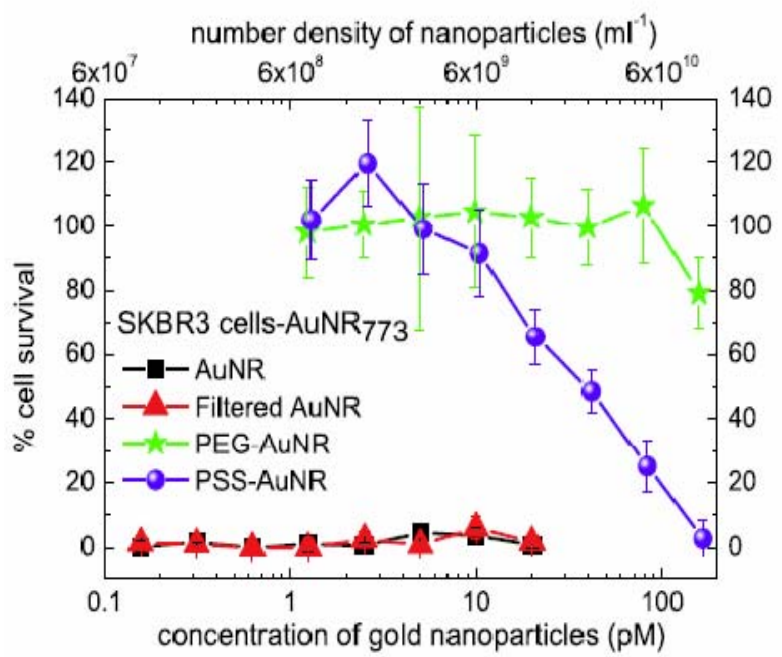

(a)

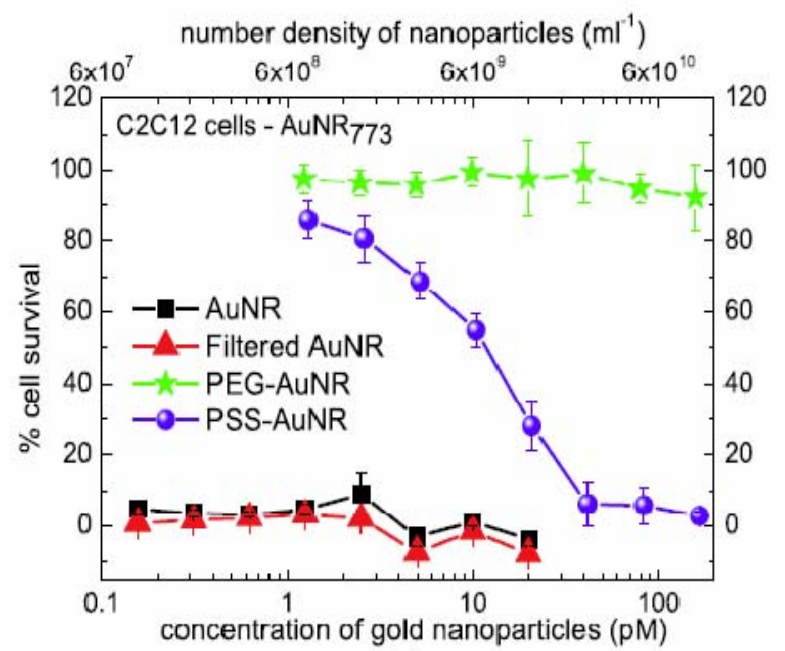

(c)

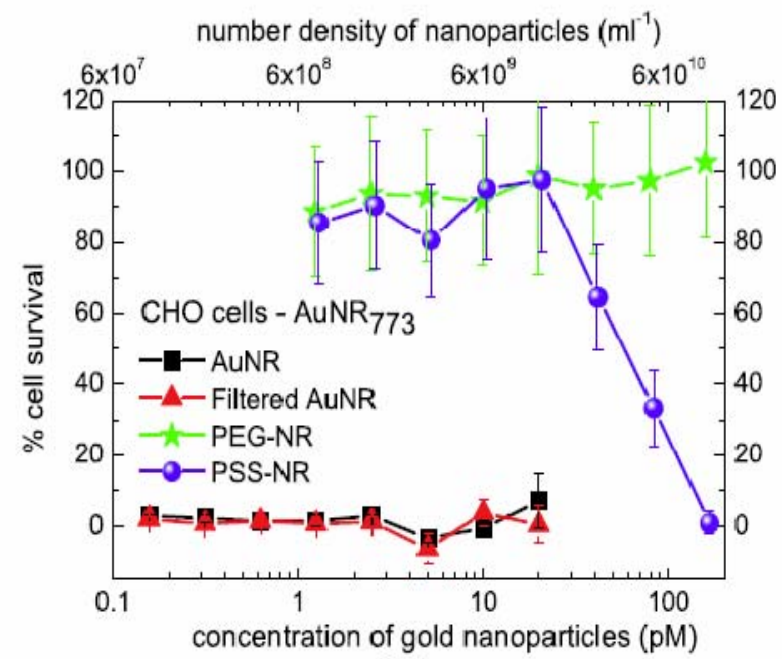

(b)

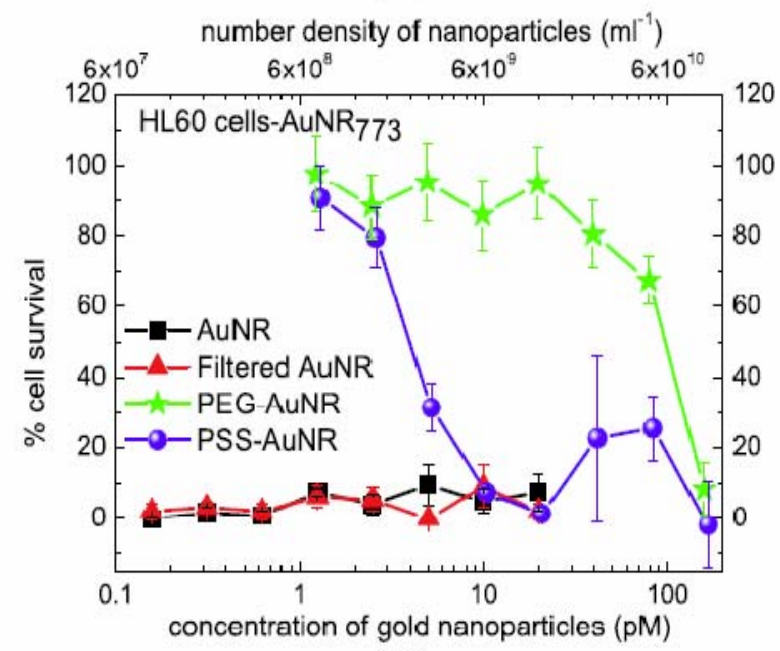

(d)

Supporting Information Figure 1: Dose-response curves of various cell-lines following exposure to various gold nanorods having longitudinal plasmon peak at $773 \mathrm{~nm}$. Particles studied are $\mathrm{AuNR}_{773}$, filtered AuNR 773 , PEGylated AuNR 773 and PSS-treated AuNR 773 . Cells used are (a) SKBR3 cells, (b) CHO cells, (c) C2C12 cells, and (d) HL60 cells. 
The median lethal concentration $\mathrm{LC}_{50}$ values for exposure under the tested conditions were ascertained from Supplementary Fig. 1(a)-(d) and are consolidated in Supporting Information Table 1. The values for PEG-AuNR ${ }_{773}$ are indeterminate at the concentrations and exposures studied for adherent cells but are around $100 \mathrm{pM}$ corresponding to a number density of $6 \times 10^{10} \mathrm{NRs} / \mathrm{ml}$ for the HL60 cells.

\begin{tabular}{|c|c|c|c|c|}
\hline Cell line & $\begin{array}{c}\text {AuNR}_{773} \\
(\mathrm{pm})\end{array}$ & $\begin{array}{c}\text { Filtered-AuNR } \\
(\mathrm{pm})\end{array}$ & $\begin{array}{c}\text { PSS-AuNR }_{773} \\
(\mathrm{pm})\end{array}$ & $\begin{array}{c}\text { PEG-AuNR }_{773} \\
(\mathrm{pm})\end{array}$ \\
\hline SKBR3 & $<0.1563$ & $<0.1563$ & 38 & $>157$ \\
\hline CHO & $<0.1563$ & $<0.1563$ & 57 & $>157$ \\
\hline C2C12 & $<0.1563$ & $<0.1563$ & 11 & $>157$ \\
\hline HL60 & $<0.1563$ & $<0.1563$ & 4 & 97 \\
\hline
\end{tabular}

Supporting Table 1: $\mathrm{LC}_{50}$ values in $\mathrm{pM}$ of unmodified and surface modified $\mathrm{AuNR}_{773}$ particles. $\mathrm{LC}_{50}$ values are determined from the dose-response curves as the concentration where there is $50 \%$ cell death.

\section{Comparison of $\mathrm{LC}_{50}$ concentrations with AuNR concentrations required for photoacoustic imaging}

The $\mathrm{LC}_{50}$ values obtained may be compared with requirements of AuNR concentrations for the specific application of photoacoustic imaging. Recently we have determined using our photoacoustic Computed Tomography (CT) mouse imager $[1,2]$ that the system is capable of detecting $3.3 \times 10^{10} \mathrm{NRs} / \mathrm{ml}$ in a $2 \mathrm{~mm}$ diameter alginate sphere. The NR-embedded sphere functioned as an inhomogeneity, embedded at the center of a $26 \mathrm{~mm}$ diameter cylindrical, tissue-mimicking phantom $[3,4]$. The concentration corresponds to a number of $138 \times 10^{6} \mathrm{NRs}$ in the sphere. In tissue, this volume would be occupied by approximately $5 \times 10^{6}$ cells - cells represented by spheres of $10 \mu \mathrm{m}$ diameter. Under the assumption that a similar concentration would also be possible in tissue, the ratio of cells to NRs would be $\sim 30$. This figure is considerably lower than the cell-NR ratios encountered in this study. For example, HL60 cells $\left(5000\right.$ cells) to PSS-AuNR $628\left(1.8 \times 10^{9} \mathrm{NR} / \mathrm{ml}\right)$ exposure at the $\mathrm{LC}_{50}$ value yield a cellNR ratio of 360,000 . 


\section{References}

1) J. Jose, R. Willemink, S. Resink, T. Maalderink, J.C.G. van Hespen, T.G. van Leeuwen, and S. Manohar, SPIE proceedings, 73710S, 2009.

2) J. Jose, T. Maalderink, G.H. Willemink, J.W. Van Neck, S. Manohar, and T.G. van Leeuwen, "A Computed Tomography photoacoustic imager for imaging murine disease models," in prep.

3) A. Kharine, S. Manohar, R. Seeton, R.G.M. Kolkman, R.A. Bolt, W. Steenbergen and F.F.M. de Mul, Phys. Med. Biol. 48, 357, 2003.

4) S. Manohar, A. Kharine, J.C.G. van Hespen, W. Steenbergen and T.G. van Leeuwen, J. Biomed. Opt. 9 , 1172, 2004. 
Chapter 6

\title{
Blood Clearance And Tissue Distribution of PEGylated and Non-PEGylated Gold Nanorods After Intravenous Administration In Rats
}

\begin{abstract}
Gold nanorods whose aspect ratios can be tuned to obtain plasmon peaks between $600 \mathrm{~nm}$ and $850 \mathrm{~nm}$ are investigated as contrast enhancing agents for diagnostic and therapeutic applications. In this study, we compared the blood clearance and tissue distribution of hexadecyltrimethylammonium bromide (CTAB) capped and poly ethylene glycol (PEG) coated gold nanorods after intravenous injection in the tail vein of rats. The gold content in blood and various organs was measured quantitatively with inductively coupled plasma mass spectrometry (ICP-MS). The CTAB capped gold nanorods were almost immediately $(<15$ minutes) cleared from the blood circulation whereas the PEGylation of gold nanorods resulted in a prolonged blood circulation with a half-life time (t1/2) of 19 hours and more wide spread tissue distribution. While for the CTAB capped gold nanorods, the tissue distribution was limited to liver, spleen and lung, the PEGylated gold nanorods also distributed to the kidney, heart, thymus, brain and testes. PEGylation of the gold nanorods resulted in the spleen being the organ with the highest exposure whereas for the nonPEGylated CTAB capped gold nanorods the liver was the organ with the highest exposure, per gram organ. In view of the time frame (up to 48 hours) of the observed presence in blood circulation, PEGylated gold nanorods can be considered to be promising candidates for therapeutic and diagnostic imaging purposes.
\end{abstract}

This chapter has been submitted to Nanomedicine for publication as "D.P.K. Lankveld $\uparrow$, R.G. Rayavarapu, P. Krystek $\ddagger$, A.G. Oomen $\dagger$, H.W. Verharen†, T.G. van Leeuwen, W.H. De Jong $\dagger$ and S. Manohar”. (†-RIVM; $\$$-MiPlaza, Philips Research). 


\section{Introduction}

Nanotechnology is a rapidly developing technology with the potential to provide our society with a wide range of products to be used in various advanced technology applications [1]. More specifically, there is a general expectation that significant progress will be made in various medical applications for diagnostic and therapeutic purposes including the potential for specific drug delivery in cancer therapy. However, besides the beneficial effects of the developed nanodevices also the potential risks should be taken into consideration [2]. For drug delivery, nanoformulations may consist of the drug itself or at least two components, one being the pharmaceutical ingredient and the other one being the nanodevice as drug carrier $[3,4,5]$. When nanoparticles are used for diagnostic or therapeutic applications, one should realize that after having fulfilled their function, the nanomaterials themselves remain and may pose a potential toxicological risk. It is therefore that for drug delivery specifically designed biodegradable nanomaterials are preferable over non-degradable solid nanomaterials, which may have the potential for local persistence. Also for diagnostic purposes, the nanomaterials should be able to be eliminated from the body either by biodegradation and metabolism or by elimination via excretion [6].

Kinetic properties are considered to be an important descriptor for potential human toxicity and thus for human health risk. In general, the kinetics of a particle consists of four processes: absorption, distribution, metabolism, and excretion (ADME). It is important to know the amount of the external exposure that is absorbed and results in internal exposure. For therapeutic and diagnostic applications, mostly a direct systemic administration into the blood circulation is used, so the whole dose applied should be taken into consideration. Potentially all organs can be reached and are thus considered to be at risk for toxic responses. However, to date most particulate materials in the systemic circulation, including nanoparticles, are taken up by the so-called reticulo-endothelial system (RES). This results in entrapment of the nanoparticles mainly in organs with a high content of mononuclear phagocytes (macrophages) such as liver and spleen [7-12]. For diagnostic and therapeutic uses, such entrapment in the liver should be avoided unless a specific aim is wanted like treatment of liver diseases. One approach may be to modify the nanoparticles in order to obtain a more specific organ or cellular targeting. The use of carbohydrate binding ligands increased cellular binding of poly(D,L-lactic-co-glycolide)acid (PLGA) nanospheres [13], whereas the use of specific antibodies enabled a more specific immunologically directed targeting of the nanoparticles $[14,15]$. Cellular entrapment in macrophages can be avoided by surface 
modification of the nanoparticles. Coupling of polyethylene glycol (PEG) onto the surface of nanoparticles was found to increase their circulation time in the blood by inhibiting recognition and phagocytosis by macrophages [16, 17]. Using PEGylation of gold nanorods an increase of gold, as measured by inductively coupled plasma mass spectrometry (ICPMS), in both spleen and tumour tissue was observed while the level in the liver was remarkably reduced [17]. In addition, the size of nanoparticles can be a significant determinant of particle distribution, as shown for spherical gold nanoparticles and gold composite nanodevices $[12,18,19]$.

For diagnostic purposes, optical imaging is currently under development for detecting, diagnosing, staging, and treatment monitoring of disease. Progression of disease is usually accompanied by changes in physiology and pathology that are manifested as location specific changes in optical properties, thereby providing contrast for optical imaging to study disease. Imaging techniques use contrast-enhancing agents of which gold nanoparticles have emerged due to their unusual optical properties and inherent biocompatibility [20, 21, 22].

Gold metal nanoparticles exhibit narrow and intense absorption and scattering bands owing to plasmon resonance, the collective oscillation that the conduction electrons experience in an electromagnetic field of the appropriate wavelength [23]. The plasmon resonant condition of gold nanoparticles depends upon their size, shape, structure (solid or hollow), and upon the refractive index of the embedding medium. Spherical gold nanoparticles have a single plasmon resonant extinction peak at around $520 \mathrm{~nm}$, which does not shift extensively with changes in size and refractive index of the surrounding medium. Due to the strong scattering and absorption by haemoglobin, at this wavelength, light penetration in tissue is poor and gold nanospheres are not useful in contrast enhancement for deep tissue imaging. Rod-shaped nanoparticles exhibit two plasmon resonances due to oscillation of the conduction electrons along the short axis as well as along the long axis of the particles. Thus by tailoring the length and/or width of these particles, their extinction peaks may be made to cover the low-energy visible to infrared wavelength regions [1, 21]. Further, gold-protein chemistry is well developed and several bioconjugation protocols are available in the literature, which allows the combination of the targeting functionality of antibodies with such gold nanoparticles. The inertness and biocompatibility of gold in general hold promise the use of gold nanoparticles for in vivo imaging and therapeutic applications. The aim of the present study was to determine the influence of PEGylation of gold nanorods on the blood clearance and tissue distribution and potential organ accumulation in the rat. In this study, we used gold nanorods 
absorbing in the NIR region of the spectrum (peak absorbing wavelength of $\sim 770 \mathrm{~nm}$ ). The PEGylated and non-PEGylated gold nanorods were intravenously injected into rats and gold content in various organs was determined using inductively coupled plasma mass spectrometry (ICP-MS).

\section{Materials, Animals and Methods}

\section{Materials}

We used hexadecyltrimethylammonium bromide (CTAB, Acros 22716V, Belgium) for both growth and seed solutions. Gold salt $\left(\mathrm{HAuCl}_{4} .3 \mathrm{H}_{2} \mathrm{O}, 99.99 \%\right)$ was purchased from Acros Organics (Belgium), sodium borohydride $\left(\mathrm{NaBH}_{4}, 99 \%\right)$, and ascorbic acid (99\%) from Sigma (The Netherlands), and silver nitrate $\left(\mathrm{AgNO}_{3}, 99.8 \%\right)$ from Merck (Germany), mercaptopolyethylene glycol monomethyl ether, PEG thiol (mPEG-SH), 5K, Fluka (The Netherlands). Prior to use, all glassware was cleaned with $8 \%$ hydrofluoric acid (HF), further with aqua regia $\left(\mathrm{HCl} / \mathrm{HNO}_{3}, 3: 1\right)$ and rinsed thoroughly with Milli Q water.

\section{Synthesis of gold nanorods (AuNRs)}

Preparation of growth solution:

To freshly prepared $0.5 \mathrm{ml}$ of $0.01 \mathrm{M}$ gold salt solution, $9.5 \mathrm{ml}$ of $0.1 \mathrm{M}$ CTAB solution was added, with thorough mixing to yield a dark yellow solution. The amount of silver nitrate used was $60 \mu \mathrm{l}$. To this $55 \mu \mathrm{l}$ of $0.1 \mathrm{M}$ ascorbic acid was added under stirring. The resultant turns colourless. Ten identical growth solutions were prepared to scale up the synthesis to have a final volume of $100 \mathrm{ml}$ suspension of nanorods.

Preparation of gold seed:

To freshly prepared $0.25 \mathrm{ml}$ of $0.01 \mathrm{M}$ gold salt solution, $9.75 \mathrm{ml}$ of $0.1 \mathrm{M} \mathrm{CTAB}$ was added under stirring. Ice-cold, freshly prepared $0.01 \mathrm{M}$ sodium borohydride solution in a volume of $0.6 \mathrm{ml}$ was added to the mixture all at once, while stirring for 2 minutes. The resultant seed solution that showed a light-brownish colour was added within about 5 minutes to the growth solution.

Growth phase:

The seed solution $(12 \mu \mathrm{l})$ was added to the growth solutions with gentle stirring. The resultants were maintained undisturbed at $25{ }^{\circ} \mathrm{C}$ for 24 hours, after which they were 
centrifuged twice at $10000 \mathrm{~g} / 20$ minutes and the supernatants removed. The precipitates were re-dispersed in phosphate buffer saline (PBS).

\section{PEGylation of AuNRs}

The gold nanorods were washed twice at $10000 \mathrm{~g} / 20$ minutes. The supernatant was discarded and to the pellet of nanorods $0.5 \mathrm{ml}$ of $\mathrm{mPEG}-\mathrm{SH}$ was added along with $0.5 \mathrm{ml}$ of PBS. The nanorods were then vortexed for 30 seconds and were kept on stirring overnight. After stirring, the nanorods were centrifuged once to remove excess of PEG thiol and next the pellet was dispersed in PBS. Before use, the amount of CTAB was reduced by dialysis against PBS to reduce toxicity due to the presence of CTAB.

\section{AuNRs characterization}

Electron microscopy of the nanoparticles was performed using a Zeiss- 1550 high-resolution scanning electron microscope (SEM). Particle sizes were estimated using the NI Vision module (Labview, National Instruments) on the digital SEM images for which approximately 250 particles were considered.

Optical spectra of nanorods were measured with collimated transmission in a Shimadzu PC3101 UV-Vis-NIR spectrophotometer. The nanorods synthesized have two plasmon peaks, a transverse plasmon peak at approximately $517 \mathrm{~nm}$ and a longitudinal plasmon peak at 770 $\mathrm{nm}$. The longitudinal plasmon peak is in the near infrared region (NIR) of the spectrum. Based on the longitudinal plasmon peak at $770 \mathrm{~nm}$ the non-PEGylated CTAB capped gold nanorods were designated $\mathrm{CTAB}-\mathrm{AuNR}_{770}$ and the PEGylated gold nanorods were designated PEG-AuNR 770 .

The concentrations of non-PEGylated and PEGylated gold nanorods were determined by the optical spectra. The extinction coefficient value of the longitudinal plasmon peak is taken into consideration for calculating the number of particles/ml. The mean length and width of gold nanorods were measured. The mass of a single gold nanorod was calculated by taking the density of gold that is multiplied with the volume of a single gold nanorod. The number of particles/ml was multiplied with the mass of gold nanorods to calculate the concentration of $\mathrm{Au}$ in $\mu \mathrm{g} / \mathrm{ml}$. 


\section{Zeta -potential measurements of AuNRs}

The zeta-potential measurements of gold nanorods were carried out using a Malvern Zetasizer Nano Z(S). The cuvettes (DTS 1060) were used for measuring the zeta potential $(\mathrm{mV})$ of nanorods. $1 \mathrm{ml}$ nanorods were filled in the cuvette using a syringe avoiding any airbubbles. The zeta-potential measurements determine the charge present on the surface of nanoparticle. The as-prepared CTAB capped gold nanorods show positive zeta potential due to the presence of CTAB bilayer on the surfaces of the gold nanorods. The PEGylation of gold nanorods causes the displacement of CTAB with PEG and hence the surface charge changes to nearly neutral. The zeta-potential of the original CTAB capped gold nanorods $\left(\mathrm{CTAB}-\mathrm{AuNR}_{770}\right)$ was $(+42 \pm 7.2) \mathrm{mV}$, while for the PEGylated gold nanorods (PEG$\left.\mathrm{AuNR}_{770}\right)$ the zeta-potential was $(0.4 \pm 2.1) \mathrm{mV}$. The results of the characterization of the gold nanorods are presented in Table 1. The optical spectra and a SEM image of the gold nanorods are presented in figure 1.

\section{Animals}

Six-week-old male Wistar rats (HsdCpb:WU) were purchased from Harlan Nederland BV (Horst, The Netherlands) and allowed to acclimatize for 2 weeks before starting the experiment. Animals were bred under specific pathogen-free (SPF) conditions and barrier maintained during the entire experiment in Macrolon cages at a room temperature of (23 \pm $1)^{\circ} \mathrm{C}$, a relative humidity of $(50 \pm 5) \%$ and a 12-hours light/dark cycle. Drinking water and conventional feed were provided ad libitum. The experiment was approved by an independent Ethical Committee on Animal Experimentation and conducted in compliance with all applicable provisions of the national laws, i.e. the Experiments on Animal Decree and the Experiments on Animal Act.

\section{Experimental design}

Rats were divided in 3 groups: non-PEGylated gold nanorods (CTAB-AuNR ${ }_{770}, \mathrm{n}=6$ ), PEGylated gold nanorods (PEG-AuNR $770, n=6)$, and PBS vehicle control $(n=3)$. Four days prior to the start of the experiment, a single venous blood sample (tail vein; $100 \mu 1$ ) was obtained from the animals in the control group. On day 0 , rats were intravenously injected (tail vein) with either $1 \mathrm{ml}$ nanogold dispersion or vehicle control. The injections were well tolerated and no adverse effects were observed during the entire experiment. Starting at day 0 venous blood samples were obtained from the treatment-groups at 0.25 (15 minutes), 0.5 (30 
minutes), 1, 2, 4, 8, 24 (day 1), 48 (day 2), 96 (day 4), and 144 (day 6) hours after injection. Blood samples from the animals in the control group were collected at day "minus" 4 (96 hours before the start of the experiment) and at day 8 (192 hours after start of the experiment). At 24 hours (day 1) and 144 hours (day 6) after the injection, rats ( $n=12,6$ per treatment group) and at 192 hours (day 8) the 3 control rats were anaesthetized with isoflurane (Isoflu ${ }^{\circledR}$, AST Pharma, The Netherlands) in oxygen and subsequently euthanized by drawing blood from the abdominal aorta. Next to blood, liver, spleen, kidneys, lungs, heart, thymus, brain and testes were collected. Organs were weighed and homogenized. All samples were stored at $-20{ }^{\circ} \mathrm{C}$ for determination of the gold content. After subjecting the sample material to a digestion process, the gold content was determined by inductively coupled plasma mass spectrometry (ICP-MS).

\section{Sample pre-treatment and inductively coupled plasma- mass spectrometry (ICP-MS)}

Reagents : All chemicals used for ICP-MS were of analytical grade or of high purity. Nitric acid $\left(\mathrm{HNO}_{3}\right)$ and hydrochloric acid $(\mathrm{HCl})$ were purchased from Merck, Germany. The calibration standard solution of gold $(\mathrm{Au})$, as well as a solution of the element rhodium $(\mathrm{Rh})$ used as internal standard, were prepared using single element stock solutions with a concentration of 1,000 $\mu \mathrm{g} / \mathrm{ml}$ from Inorganic Ventures, supplied by Instrument Solutions, The Netherlands. Deionised water $\left(\mathrm{H}_{2} \mathrm{O}\right)$ with $17.2 \mathrm{M} \Omega \mathrm{cm}$ was produced by a system from Christ, The Netherlands.

Instrumentation: A drying cabinet, from Heraeus, type T6060 (supplied by Thermo, The Netherlands) was used. For the digestion of the sample materials, a Stuart block heater SBH200D (supplied by Omnilabo, The Netherlands) was used. The presence of gold was measured with an inductively coupled plasma mass spectrometer (ICP-MS, type ELAN DRC Plus, Perkin Elmer, The Netherlands).

Accuracy and quality control aspects of the analytical procedure: Next to the control of chemical blanks, experiments were included to cover different performance characteristics and quality control aspects. However, for gold (nanoparticles) in biological materials there is no suitable reference material commercially available. The matrix effects were studied by the standard addition experiments and calculation of the recovery. These standard addition experiments were carried out by spiking blood samples of non-treated control rats with the nanoparticles (PEG-AuNR 770 and CTAB-AuNR 770 ) prior to sample pre-treatment and 
analysis. For gathering details about inhomogeneity in the sample material, four blood samples were analysed as independent duplicates (sub-samples A and B).

Sample preparation: To the homogenized organ samples $(0.2-0.5 \mathrm{~g}) 0.5 \mathrm{ml}$ concentrated $\mathrm{HNO}_{3}$ was added. The samples were placed in the drying cabinet at $50^{\circ} \mathrm{C}$ for 1 hour to predissolve the sample material. Then, the samples were transferred into digestion vials by adding three times $0.5 \mathrm{ml}$ concentrated $\mathrm{HCl}$. For blood, the sample amount was $\sim 0.3 \mathrm{~g}$ blood, to which $2 \mathrm{ml}$ aqua regia was added. The mixture was digested overnight with a maximum temperature of $125^{\circ} \mathrm{C}$. Afterwards the mixture was transferred into another vial, spiked with the internal standard $\mathrm{Rh}$ (final concentration: $100 \mu \mathrm{g} / \mathrm{L}$ ) and $\mathrm{H}_{2} \mathrm{O}$ was added to a total volume of $10 \mathrm{ml}$. The total solution was shortly shaken by hand.

ICP-MS measurement: The quantification was carried out by external calibration with internal standard correction. Stock solutions were diluted with diluted (1:5) aquaregia to relevant concentration levels. All measurements of standards and samples were carried out with the ICP-MS system. The main instrumental operating conditions were as follows: RF power $1450 \mathrm{~W}$ and nebulizer gas flow $0.91 \mathrm{~L} / \mathrm{min}$ Air. The following isotopes were measured: ${ }^{197} \mathrm{Au}$ and ${ }^{103} \mathrm{Rh}$ as internal standard.

\section{Results}

\section{Optical spectroscopy and SEM imaging}

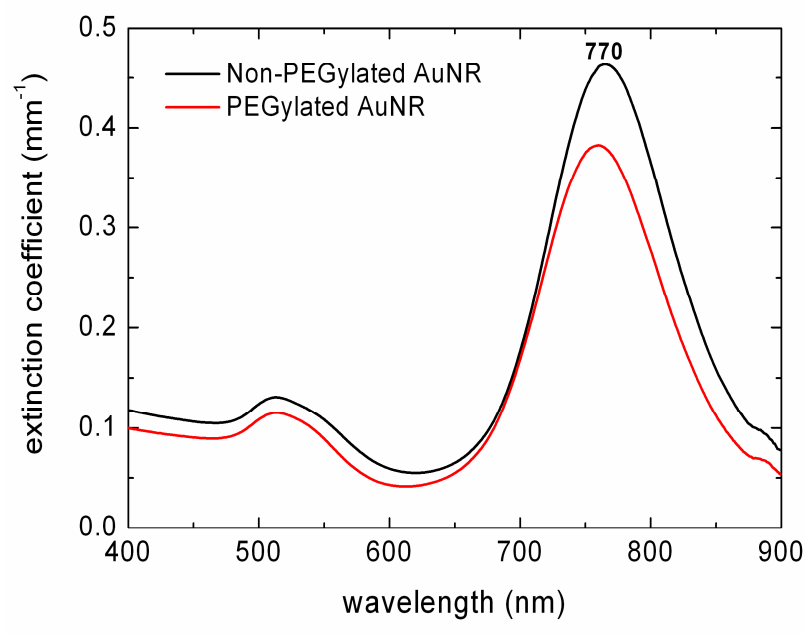

(a)

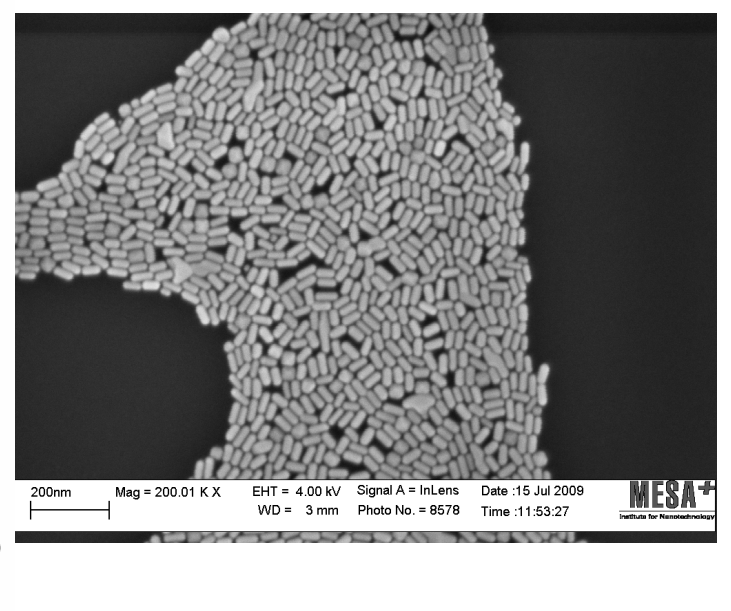

(b)

Figure 1: (a) Optical extinction spectra of the batch of gold nanorods with longitudinal plasmon peak at 770 $\mathrm{nm}$. The longitudinal plasmon peaks (black and red) correspond to as-prepared CTAB capped AuNR (NonPEGylated) and PEGylated gold nanorods respectively (b) Scanning Electron Micrograph (SEM) of the same batch showing monodispersity of gold nanorods. 


\section{Characteristics of gold nanorods used for biodistribution studies in rat organs}

\section{Samples}

Size given as mean $(\mathrm{nm})$

Aspect ratio

Number concentration $(\mathrm{NRs} / \mathrm{ml})$

Mass concentration $(\mu \mathrm{g} / \mathrm{ml})$

Zeta potential $(\mathrm{mV})$

$$
\text { (CTAB-AuNR } 770 \text { ) }
$$

3

$1.2 \times 10^{11}$

30.5

$+42 \pm 7.2$
(PEG-AuNR 770 )

$55.3 \times 18.5$

3

$9.8 \times 10^{10}$

25

$+0.4 \pm 2.1$

Table 1: Characteristics of gold nanorods before/after polyethylene glycol (PEG) coating.

\section{Blood clearance}

The blood clearance of the intravenously administered gold nanorods is presented in Table 2, Figures 2 and 3.

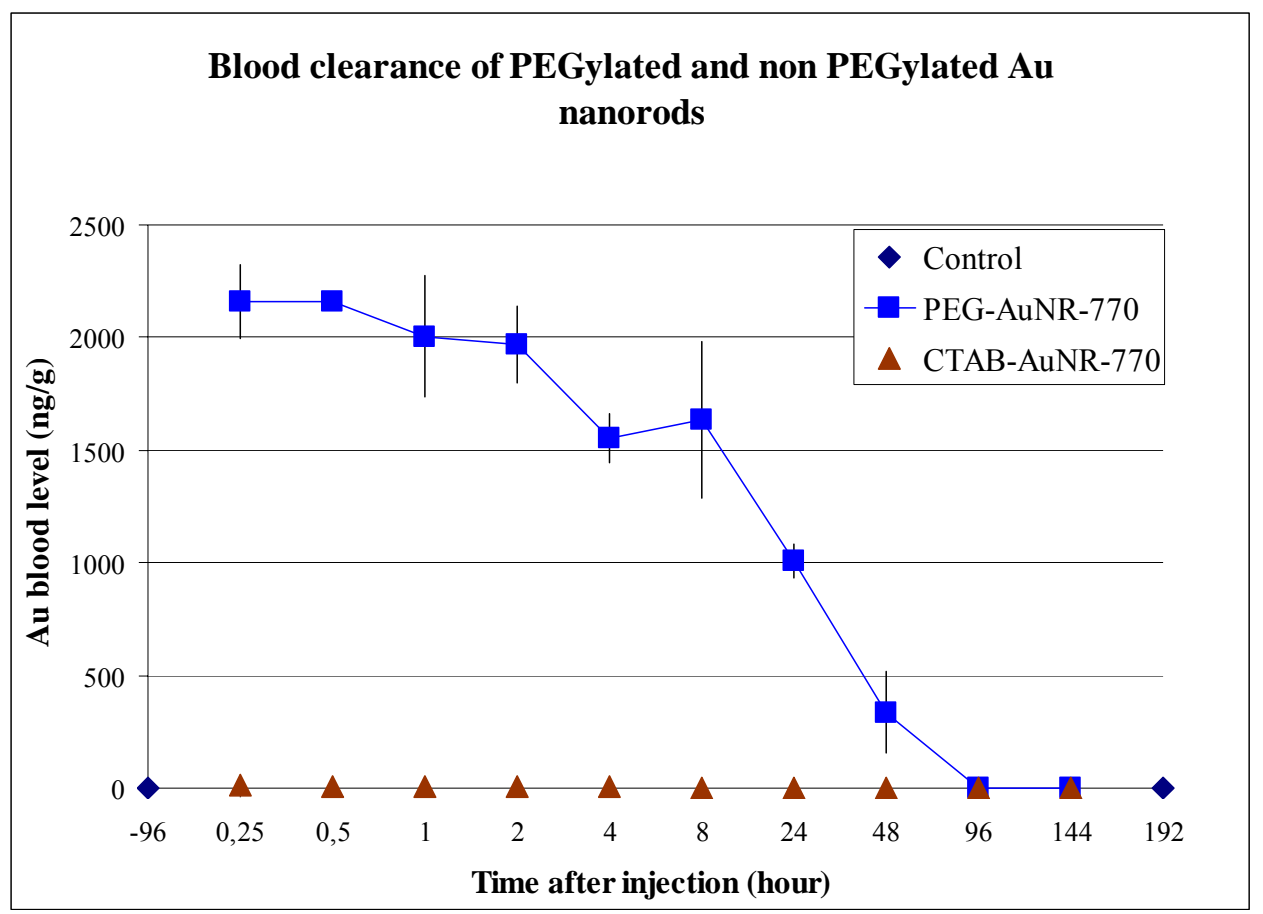

Figure 2: Blood clearance of PEGylated (PEG-AuNR 770 ) and non-PEGylated (CTAB-AuNR ${ }_{770}$ ) gold nanorods.

The results show an almost immediate clearance of the CTAB-AuNR ${ }_{770}$ from the circulation as at 15 minutes after the administration barely detectable levels of gold were measured in the blood (Table 2 and Figure 2). 


\begin{tabular}{|c|c|c|c|}
\hline Time (h) & Control & CTAB-AuNR 770 & PEG-AuNR $_{770}$ \\
\hline-96 & $3 \pm 0(3)$ & - & - \\
\hline 0.25 & & $14 \pm 8(2)$ & $2160+161(2)$ \\
\hline 0.5 & & $8 \quad(1)$ & 2157 \\
\hline 1 & & $4 \pm 1(3)$ & $2005+368(3)$ \\
\hline 2 & & $8 \pm 6(3)$ & $1967 \pm 169(3)$ \\
\hline 4 & & $4 \pm 1(3)$ & $1552 \pm 110(3)$ \\
\hline 8 & & $3 \pm 0$ & $1636 \pm 349$ \\
\hline 24 & & $3 \pm 0$ & $1007 \pm 76$ \\
\hline 48 & & $3 \pm 0(6)$ & $337 \pm 182(6)$ \\
\hline 96 & & $3 \pm 0(6)$ & $3 \pm 0$ \\
\hline 144 & & $3 \pm 0(6)$ & $3 \pm 0.5(6)$ \\
\hline 192 & $3+0(3)$ & & \\
\hline
\end{tabular}

Table 2: Blood values of gold nanorods after intravenous administration in rats.

For the PEGylated PEG-AuNR 770 blood, gold levels remain detectable until 48 hours after the administration (Table 2 and Figure 2).

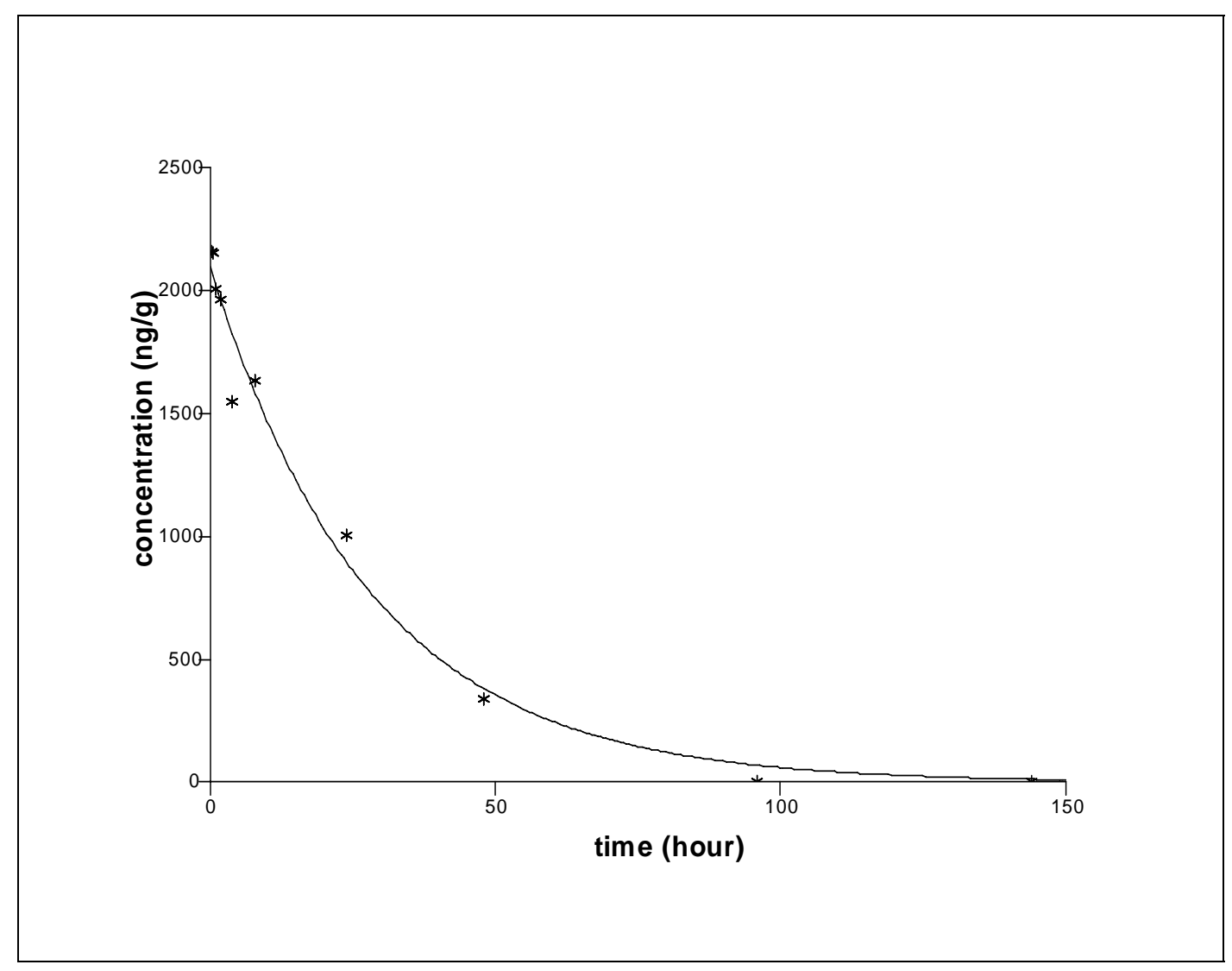

Figure 3: Fitted mono-exponential model of blood clearance of PEGylated gold nanorods (PEG-AuNR 770 ) 
Figure 3 presents a description of the blood clearance in a mathematical model. Starting with a bi-exponential model, it immediately appeared that the concentration-time course could well be described with a mono-exponential model:

$$
C(t)=C_{0} \exp (-k \cdot t)
$$

Fitting this model to the data the values $C_{0}=2100( \pm 50) \mathrm{ng} / \mathrm{g}$ and $k=0.036( \pm 0.004)-/ \mathrm{h}$ were found. The corresponding half-life time in blood $t_{1 / 2}=\ln (2) / k$ is 19 hours with a $95 \%$ confidence interval of 16 to 25 hours. The starting concentration of $2100 \mathrm{ng} \mathrm{Au} / \mathrm{g}$ blood in rats of 300 grams containing 18 grams of blood $(60 \mathrm{~g} / \mathrm{kg}$ body weight) results in a calculated administered dose of $37.8 \mu \mathrm{g}$.

\section{Tissue distribution of AuNRs}

The gold concentrations were measured in blood, liver, lungs, spleen, brain, heart, thymus, kidneys and testes at day 1 ( $\mathrm{t}=24$ hours $)$ and at day $6(\mathrm{t}=144$ hours $)$ after intravenous administration. In control rats $(n=3)$ the gold levels were at or below the detection limit (data not shown).

The gold content in tissues per gram organ is presented in Table 3.

\begin{tabular}{lcccc}
\hline \multicolumn{2}{c}{ DAY 1 } & \multicolumn{2}{c}{ DAY 6 } \\
& PEG-AuNR 770 & CTAB-AuNR 770 & PEG-AuNR 770 & CTAB-AuNR 770 \\
\hline Liver & $320 \pm 105$ & $2339 \pm 390$ & $978 \pm 145$ & $2059 \pm 299$ \\
Spleen & $3477 \pm 153$ & $1643 \pm 236$ & $6644 \pm 1973$ & $1132 \pm 204$ \\
Kidney & $183 \pm 32$ & $13 \pm 1$ & $176 \pm 29$ & $5 \pm 3$ \\
Lung & $264 \pm 22$ & $239 \pm 102$ & $106 \pm 17$ & $172 \pm 99$ \\
Heart & $192 \pm 5$ & $3 \pm 1$ & $104 \pm 13$ & $4 \pm 3$ \\
Thymus & $66 \pm 19$ & $2 \pm 0$ & $66 \pm 26$ & $2 \pm 0$ \\
Brain & $27 \pm 3$ & $5 \pm 6$ & $2 \pm 0$ & $2 \pm 1$ \\
Testes & $33 \pm 10$ & $2 \pm 0$ & $23 \pm 6$ & $2 \pm 0$ \\
Blood & $1007 \pm 76$ & $3 \pm 0$ & $3 \pm 1$ & $3 \pm 0$ \\
\hline Data are presented as gold concentration in ng/ gram tissue. Gold nanorods were administered intravenously at day 0. Number of animals (samples) $\mathrm{n}=3$ \\
for day 1 and $\mathrm{n}=6$ for day 6 . Tissue samples were prepared by organ digestion before ICP-MS measurement.
\end{tabular}

Table 3: Gold content in organs per gram tissue as determined by ICP-MS in organs of rats after intravenous administration of PEGylated and non-PEGylated gold nanorods.

The results show that the non-PEGylated CTAB-AuNR ${ }_{770}$ are mainly found in the liver and spleen, and to a lesser extent in the lung. Similar levels are observed on both day 1 and day 6 
after administration. Expressed per gram organ the highest exposure was observed in the liver, followed by spleen and lung. For the PEGylated nanorods (PEG-AuNR 770 ) in most organs at $\mathrm{t}=24$ hours and $\mathrm{t}=144$ hours gold could be detected with the highest levels on day 1 in spleen, blood and liver. At day 6, clearly accumulation in all organs was observed with the highest level in the spleen followed by the liver (Table 3).

When we consider the total levels of gold in the whole organ for the PEGylated gold nanorods at day 1, the highest levels are in the blood followed by liver and spleen (Table 4). In most organs investigated gold could be detected indicating the wide spread distribution of the PEGylated gold nanorods in the body with the highest level per whole organ in the liver (Table 4).

\section{DAY 1}

DAY 6

PEG-AuNRT70 CTAB-AuNR PEG-AuNR $770 \quad$ CTAB-AuNR 770

$\begin{array}{lcccc}\text { Liver } & 4127 \pm 1282 & 28372 \pm 3999 & 12454 \pm 960 & 26535 \pm 3954 \\ \text { Spleen } & 2183 \pm 261 & 978 \pm 181 & 4112 \pm 959 & 701 \pm 126 \\ \text { Kidney } & 362 \pm 77 & 26 \pm 3 & 360 \pm 50 & 10 \pm 6 \\ \text { Lung } & 350 \pm 23 & 293 \pm 113 & 139 \pm 20 & 215 \pm 122 \\ \text { Heart } & 182 \pm 13 & 3 \pm 1 & 99 \pm 14 & 4 \pm 3 \\ \text { Thymus } & 40 \pm 17 & 1 \pm 0 & 37 \pm 11 & 2 \pm 1 \\ \text { Brain } & 47 \pm 6 & 10 \pm 12 & 4 \pm 0 & 4 \pm 2 \\ \text { Testes } & 99 \pm 33 & 6 \pm 0 & 75 \pm 20 & 6 \pm 1 \\ \text { Blood } & 18042 \pm 1411 & 53 \pm 2 & 63 \pm 10 & 59 \pm 2\end{array}$

Data are presented as gold concentration in $n g /$ organ. Gold nanorods were administered intravenously at day 0 . Number of animals (samples) $n=3$ for day 1 and $n=6$ for day 6 .

Table 4: Gold content in organs of rats after intravenous administration of PEGylated and non-PEGylated gold nanorods.

Both at day 1 and day 6 for the non-PEGylated gold nanorods the highest levels are present in the liver at similar magnitude. In addition, also in the lung gold could be clearly detected. Table 5 and Figure 4 show the total recovery in organs when compared to the administered dose for day 1 and day 6 .

\begin{tabular}{lll}
\hline & PEG-AuNR & CTAB-AuNR \\
\hline Day 1 & $25.43 \mu \mathrm{g}(101.7 \%)$ & $29.67(97.3 \%)$ \\
Day 6 & $17.28 \mu \mathrm{g}(69.1 \%)$ & $27.47 \mu \mathrm{g}(90.1 \%)$ \\
\hline
\end{tabular}

Table 5: Total recovery of gold content in organs as percentage of administered dose. 
At day 0 the gold nanorods were intravenously injected. PEG-AuNR-770 as $25 \mu \mathrm{g}$ per animal, and CTABAuNR-770 as $30.5 \mu \mathrm{g}$ per animal.

The recovery percentages of gold per organ as percentage administered on day 1 and day 6 in several organs of rat are shown in Figure 4.
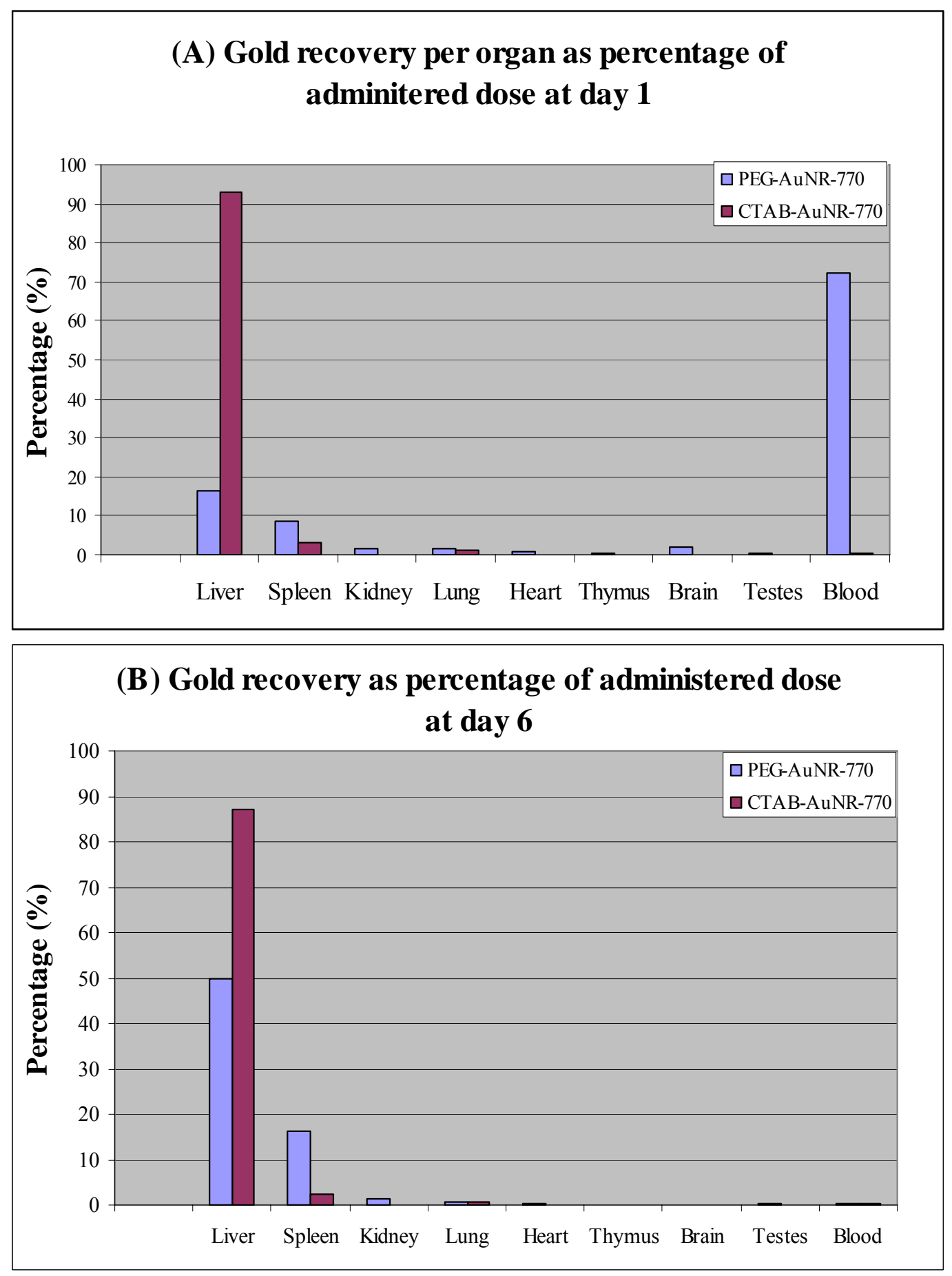

Figure 4: Recovery of gold as percentage of administered dose into rat. 
The total recovery varied from approximately $70 \%$ to more than $100 \%$. However, the recovery might be overestimated. Based on the calculations for the blood clearance for the PEG-AuNR 770 at a starting dose of $37.8 \mu \mathrm{g}$ was calculated. The dose administered according to Table 1 based on optical density calculations indicate a concentration per $\mathrm{ml}$ and thus dose per animal of $25 \mu \mathrm{g}$. ICP-MS measurement of the PEG-AuNR ${ }_{770}$ showed a concentration of $34.5 \mu \mathrm{g}$ that was more in line with the figure calculated from the blood concentrations (data not shown).

\section{Discussion}

We investigated the blood clearance, tissue distribution of two types of gold nanorods, CTAB capped gold nanorods, and PEGylated gold nanorods, specifically designed as potential probes for light based imaging techniques [1]. The main finding was the remarkable contrast in blood clearance between the PEGylated and non-PEGylated gold nanorods. The nonPEGylated CTAB capped gold nanorods were almost directly removed from the blood circulation, whereas for the PEGylated gold nanorods a blood half time value of 19 hours was observed. The non-PEGylated gold nanorods were sequestered to the liver mainly, and to a lesser extent to the spleen followed by the lung. When expressed as dose per gram organ the highest exposure was observed in the liver. Although for diagnostic use the clearance of imaging contrast agents has to be reasonably fast, the very quick disappearance as seen for the non-PEGylated CTAB capped gold nanorods also greatly diminishes its potential applications. In this respect, the PEGylated gold nanorods show more promise as they remain in circulation for some time. The half time of 19 hours allows the nanorods to reach all organs in the body thus widening the possible use as imaging agent. Our results on the prolonged circulation of the PEGylated gold nanorods and the very quick disappearance from the circulation of the non-PEGylated gold nanorods are in agreement with previous reports on the blood clearance of gold nanorods [16]. For avoidance of sequestration by the RES the amount of PEG grafted on the nanorods is of importance [16]. Our results indicate that sufficient PEG was present on the gold nanorods for a considerable increase in the circulation time.

In terms of potential toxicity, the exposure per gram organ is relevant. In absolute amount most of the gold was found to be present in the liver on both day 1 and day 6 after administration. When we consider the cellular exposure of the organs by evaluation of the concentration per gram organ for the PEGylated gold nanorods the highest exposure was in 
the spleen whereas the highest exposure for the non-PEGylated gold nanorods was in the liver. The uptake of nanoparticles by mainly liver and spleen and a size-related distribution over these organs is also described in [5].

Tissues with a fenestrated or incomplete endothelial lining, such as liver and spleen, respectively, were suggested to contribute to a significant uptake of nanoparticles. Moreover, it was concluded that particles smaller than the pore size of liver fenestrae $(\sim 100 \mathrm{~nm})$ lead to enhanced liver uptake, whereas larger particles lead to enhanced spleen uptake [5]. Most types of nanomaterials including soft nanomaterials like liposomes mainly end up in liver and spleen as major organs of the reticulo-endothelial system (RES) that is dedicated to removal of agents from the blood. Our results indicate that the non-PEGylated gold nanorods are quickly taken up primarily by the liver and the spleen with the highest concentration per gram organ in the liver, whereas the prolonged circulation of the PEGylated gold nanorods results in a much higher concentration per gram organ in the spleen. The prolonged circulation was of limited duration as eventually most PEGylated gold nanorods ended in the liver and spleen. However, it resulted in the distribution beyond the liver and spleen, and resulted in the highest tissue concentration in the spleen instead of the liver. As the gold nanorods were of the similar size the size could not be responsible for the altered distribution between liver and spleen.

Besides the liver and spleen other organs with detectable gold levels were kidney, lung, heart, and to a lesser extent thymus, brain and testes. For the brain, the content dropped below the detection level at day 6 after administration. However, we have to realise that low levels expressed as mass or as percentage of the injected dose still may be a considerable amount of nanoparticles. When we look at the $1.9 \%$ of the injected dose present in the brain at day 1 after injection, this translates to a number of $2 \times 10^{10}$ AuNRs based on the data presented in Table 1. When local accumulation in organs would occur this low fraction of the injected dose still may be the cause for a delayed toxic response. The percentage recovery of the injected dose was for the PEGylated gold nanorods for other organs than liver and spleen below $2 \%$ at day 1 , which decreased further below $1 \%$ at day 6 , indicating the washing out of the gold nanorods. In liver and spleen there was a considerable increase in gold content when comparing the gold level at day 6 with day 1.

At autopsy as much blood as possible was collected to maximize removal of residual blood from the organs. The amount of gold in residual blood is included in the measured tissue 
concentrations. This should be especially considered for the PEGylated gold nanorods as these remain in circulation for a prolonged time. Hence, tissue levels could have been overestimated. However, an organ with high-blood content like the spleen shows at day 1 a higher gold concentration than blood, suggesting that residual blood could have had only a minor effect on the amount of gold measured. On the other hand, the concentrations found in the other organs (liver, kidney, lung, heart, thymus, brain, and testes) were below the concentration in blood. Therefore, contribution of residual blood cannot be ruled out for these organs. For the measurements of the non-PEGylated gold nanorods and the day 6 measurements, blood levels are too low to have an effect on the tissue measurements. The day 6 measurements clearly show the difference in tissue distribution between the PEGylated and non-PEGylated CTAB capped gold nanorods, with the PEGylated gold nanorods showing a more widespread tissue distribution.

The distribution of the gold nanorods in blood and tissue was determined by measuring the gold content of tissue samples by ICP-MS, a method commonly used for the determination of the tissue levels of gold nanoparticles $[12,17]$. Other methods used for following the fate of gold nanoparticles are neutron activation either before use [19] so the radionucleide $\mathrm{Au}^{197}$ or $\mathrm{Au}^{198}$ was administered, or afterwards in the tissue samples by instrumental neutron activation analysis (INAA) [18]. Although the method of neutron activation allows the determination of the gold nanoparticles themselves when the activation of the nanogold preparation was done before the administration to the animals, it also requires specific infrastructures that are not easily available. For INAA the same applies as for ICP-MS that the total gold content is determined and not specifically the gold nanoparticles. For ICP-MS measurement, sample preparation (digestion) is needed with e.g. the possibility of loss of the sample due to the used procedure. Determination of the tissue distribution of injected materials is also possible with the use of a radioactive label, which, depending on the label, can be used with a more limited processing method that may limit loss of test sample. For more complex composed nanoparticles there is, however, the possibility for losing the label, as was previously demonstrated for technetium labeled carbon particles used for studying lung passage of inhaled ultrafine particles $[24,25,26]$. As the degradation of the gold seems unlikely, the determination of the gold content in organs by ICP-MS is a good measure for the tissue distribution of gold nanomaterials such as gold nanoparticles and nanorods. 


\section{Conclusions}

We demonstrated that the coating of gold nanorods with PEG had a significant effect on both the blood clearance and tissue distribution of the gold nanorods. PEGylation of gold nanorods resulted in a prolonged blood circulation with a half-life time of 19 hours and more wide spread tissue distribution. PEGylation of the gold nanorods resulted in the spleen being the organ with the highest exposure whereas for the non-PEGylated CTAB capped gold nanorods the liver was the organ with the highest exposure. In view of the time frame (up to 48 hours) of the observed increase in blood circulation PEGylated gold nanorods can be considered to be promising candidates for diagnostic imaging purposes.

\section{Acknowledgements}

We thank Prof. Vinod Subramaniam (University of Twente, BPE) for initiating this work with RIVM. Ron Vlug, Hans Strootman, Liset de la Fonteyne, Nick van Oyen (all from RIVM), and Amanda Troost-De Jong and Jeannette Smulders (both from MiPlaza) are acknowledged for their excellent technical support during the study. Jan Van Eijkeren (RIVM) is acknowledged for the mathematical modelling of the data.

\section{References}

1) R.G. Rayavarapu, W. Petersen, C. Ungureanu, J.N. Post, T.G. van Leeuwen and S. Manohar, IJBI, 29817, 2007.

2) W.H. De Jong and P.J.A. Borm, Int. J. Nanomed, 3, pp. 133-149, 2008.

3) R. Duncan, Nat Rev Drug Disc, 2, pp. 347-360, 2003.

4) M. Ferrari, Nat Rev Cancer, 5, pp. 161-171, 2005.

5) S.D. Li and L. Huang, Mol Pharmac., 5, pp. 496-504, 2008.

6) F. Alexis, E. Pridgen, L.K. Molnar and O.C. Farokhzad, Mol Pharmac, 5, pp. 505-515, 2008.

7) V. Lenaerts, J.F. Nagelkerke, T.J. Van Berkel, P. Couvreur, L. Grislain, M. Roland and P. Speiser, J. Pharm. Sci. 73, pp. 980-982, 1984.

8) S. Ginaud, M. Demoy, J.P. Andreux, C. Weingarten, B. Gouritin and P. Couvreur, J. Pharm. Sci., 85, pp. 944-950, 1996.

9) M. Demoy, S. Gibaud, J.P. Andreux, C. Weingarten, B. Gouritin and P. Couvreur, Pharm. Res. 14, pp. 463-468, 1997.

10) S.M. Moghimi, A.C. Hunter and J.C. Murray, Pharmacol Rev, 53, pp. 283-318, 2001.

11) E. Sadauskas, H. Wallin, M. Stoltenberg, U. Vogel, P. Doering, A. Larsen and G. Danscher, Part Fibre Toxicol, 4, 2007.

12) W.H. De Jong, W.I. Hagens, P. Krystek, M.C. Burger, A.J. Sips and R.E. Geertsma, Biomaterials, 29, pp. 1912-1919, 2008.

13) A. Weissenbrock, M. Wirth and F. Gabor, J. Contrl. Release.,99, pp. 383-392, 2004.

14) L. Nobs, F. Buchegger, R. Gurny and E. Allémann, Eur. J. Pharm. Biopharm., 58, pp. 483-490, 2004.

15) L. Prinzen, R. Miserus, A. Dirksen, T.M. Hackeng, N. Deckers, N.J. Bitsch, R.T. Megens, K. Douma, J.W. Heemskerk, M.E. Kooi, P.M. Frederik, D.W. Slaaf, M.A. van Zandvoort and C.P. Reutelingsperger, Nano. lett., 7, pp. 93-100, 2007.

16) T. Niidome, M. Yamagata, Y. Okamoto, Y. Akiyama, H. Takahishi, T. Kawano, Y. Katayama and Y. Niidome, J. Contl. Release, 114, pp. 343- 347, 2006.

17) Y. Akiyama, T. Mori, Y. Katayama, and T. Niidome, J. Contrl. Release. 139, pp. 81-84, 2009. 
18) L. Balogh, S.S. Nigavekar, B.M. Nair, W. Lesniak, C. Zhang, L.Y. Sung, M.S.T. Kariapper, A. ElJawahri, M. Llanes, B. Bolton, F. Mamou, W. Tan, A. Hutson, L. Minc and M.K. Khan, Nanomedicine: Nanotechnol, Biol and Medicine, 3, pp. 281-296, 2007.

19) M. Semmler-Behnke, W.G. Kreyling, J. Lipka, S. Fertsch, A. Wenk, S. Takenaka, G. Schmid and W. Brandau. Small, 4, pp. 2108-2111, 2008.

20) A.W.H. Lin, N.A. Lewinski, J.L. West, N.J. Halas and R.A. Drezek, J. Biomed. Optics, 10, 064035, 2005.

21) J. Perez-Juste, I. Pastoriza-Santos, L. Liz-Marzan and P. Mulvaney, Coord. Chem. Rev. 249, pp. 18701901, 2005.

22) K. Sokolov, M. Follen, J. Aaron, I. Pavlova, A. Malpica, R. Lotan and R. Richards-Kortum, Cancer Res. 63, pp. 1999-2004, 2003.

23) D.A. Stuart, A.J. Haes, C.R. Yonzon, E.M. Hicks and R.P. Van Duyne, IEEE Proc. Nanobiotechnol, 152, pp. 13-32, 2005.

24) A. Nemmar, P.H. Hoet, B. Vanquickenborne, D. Dinsdale, M. Thomeer, M.F. Hoylaerts, H. Vanbilloen, L. Mortelmans and B. Nemery, 105, pp. 411-414, 2002.

25) W.G. Kreyling, M. Semmler and W. Moller, J. Aerosol. Med., 17, pp. 140-152, 2004.

26) N.L. Mills, N. Amin, S.D. Robinson, A. Anand, J. Davies, D. Patel, J.M. de la Fuente, F.R. Cassee, N.A. Boon, W. Macnee, A.M. Millar, K. Donaldson and D.E. Newby, Am J. Respi. Crit. Care Med., 173, pp. 426-431, 2006. 
Chapter 7

\title{
Antibody conjugation to gold nanorods via PEG linkers: in vitro toxicity and cellular uptake studies in breast adenocarcinoma cell line
}

\begin{abstract}
Gold nanoparticles exhibit intense and narrow optical extinction bands due to the phenomenon of plasmon resonance making them useful as contrast agents for light-based imaging techniques. Localized heating results from the absorbed light energy, which makes these nanoparticles potentially useful as photothermal therapeutic agents as well. The bioconjugation of gold nanoparticles to appropriate antibodies targeted to tumors could make highly selective in vivo detection and therapy of tumors possible. We have synthesized gold nanorods (AuNR) using the seed-mediated surfactant method having longitudinal plasmon peak at $628 \mathrm{~nm}$. The $\mathrm{AuNR}_{628} \mathrm{~nm}$ that were used in this study have aspect ratio of 2.3 . Typical concentrations of these particles in aqueous dispersions are $1 \times 10^{11} \mathrm{NRs} / \mathrm{ml}$. To provide targeting capability we have bioconjugated gold nanorods covalently via thiol linkages using a heterobifunctional PEG linker (OPSS-PEG-NHS), to HER81 mouse monoclonal antibodies (mAb). The toxicity of these antibody conjugated gold nanorods to SKBR3 cells for two incubation periods was assessed using a propidium iodide (PI) assay. Characterization and size estimation of the nanoparticles were performed using electron microscopies, optical spectroscopy and confocal microscopy. Cellular uptake and intracellular transport of these nanorods was also studied using Transmission Electron Microscopy.
\end{abstract}

This manuscript will be communicated as "R.G. Rayavarapu, W. Petersen, Patrick Chin†, Hans Janssen†, F.W.B. van Leeuwen†, T.G. van Leeuwen and S. Manohar”. (†- Netherlands Cancer Institute). 


\section{Introduction}

The strong surface plasmon resonances of gold nanoparticles at optical frequencies make them excellent scatterers and absorbers of light [1]. The notable use of gold nanoparticles has been as contrast agents in cellular and biomedical imaging $[2,3]$. The purpose of these contrast agents is to improve the sensitivity and specificity of the measuring modality by sitespecifically labeling tissues or cells of interest. Rod-shaped gold nanoparticles have unique optical properties $[4,5]$ possessing two plasmon peaks in contrast to the solitary peak exhibited by gold nanospheres. The transverse plasmon (TP) peak is seen in the visible region $(520 \mathrm{~nm})$ due to the excitation of plasmons along the short axis of the nanorod, and a reddened longitudinal plasmon (LP) peak due to creation of plasmons along the long axis of the nanorod. The LP peak of gold nanorods can be red-shifted by increasing the asymmetry, in the form of the aspect ratio of the particle. For use in light-based imaging or therapy in tissues, contrast agents should have strong optical absorption and/or scattering in the NIR part of the spectrum where penetration of light is high. In the wavelength regime between $600 \mathrm{~nm}$ and $950 \mathrm{~nm}$, absorption and scattering losses are minimal permitting high light penetration. This is the so-called "optical imaging window" which is exploited for deep imaging in tissue. To use gold nanorods for the purpose of contrast enhancement in imaging, the appropriate aspect ratios should be synthesized that place the longitudinal peak in this range. Further, for in vivo use, the nanoparticles and their chemical constituents should be biocompatible which could require modification of their surfaces. To impart specificity to the disease-state such as cancer, that is being imaged or treated, targeted delivery is a key parameter [6]. This can achieve by conjugating the particles with proteins such as antibodies or peptides having affinity for disease markers.

Earlier we showed that as-synthesized CTAB capped AuNRs are toxic to cells $[7,8]$. The toxicity was mitigated by treating the nanorods with PEG-thiol (m-PEG-SH), which results in strong sulphur binding of the polymer to the gold surface with replacement of (most of) the toxic CTAB bilayer. The resulting nanorods were shown to be relatively non-toxic but also colloidally stable even in the presence of salts in cell culture medium. A logical further step from the detoxification and stabilization function of PEG is to use the polymer as a linker via the second terminus to an antibody that can bind to the receptors on the tumour cell.

The literature has reported on bioconjugation of gold nanoparticles with affinity biomolecules, using non-covalent means $[4,5]$ and covalent means $[9,10]$. The non-covalent conjugation of proteins to colloidal gold is usually through a combination of electrostatic and 
hydrophobic interactions. Positively charged amino groups of the antibody will be attracted to the gold surface, and when the protein comes close enough for binding, the hydrophobic pockets of the protein will make contact and bind with the gold. Using this method, we conjugated AuNRs with HER81 antibody, which bind with high efficiency to HER2 receptors expressed by SKBR3 breast carcinoma cells. We demonstrated in fixated cell studies that the targeting functionality of the antibody moiety remains viable. However, the method of non-covalent binding is expected to be less strong and less stable compared with the covalent coupling strategies, which is a drawback especially in the hostile environments found in vivo.

Huff et al [11] used a heterobifunctional cross-linker LC-SPDP to conjugate secondary antibodies to the nanorod surface using the covalent binding of the pyridildithio group with the gold nanorod surface. Recently, Eghtedari et al [12] reported covalent bioconjugation of gold nanorods using Nanothinks acid activated by EDC-NHS to couple to antibodies.

In this chapter, we report the covalent (thiol linkage) bioconjugation of gold nanorods using a heterobifunctional PEG linker (OPSS-PEG-NHS) to the HER81 antibody. This strategy was inspired by the work of Loo et al [10] who used the approach to couple gold nanoshells to HER2 antibody. We present immunostaining studies using the SKBR3 breast adenocarcinoma cell-line, demonstrating the success of the bioconjugation and the retention of the functionality of the antibody. Further, we show the results of toxicity studies of the antibody conjugated AuNRs to the SKBR cells, and cellular uptake using optical and electron microscopy.

\section{Materials and Methods:}

Tetrachloroauric acid $\left(\mathrm{HAuCl}_{4} .3 \mathrm{H}_{2} \mathrm{O}\right)$ was purchased from Acros Organics (Belgium), hexadecyltrimethylammonium bromide (CTAB; > $99 \%$ ) and PEG-thiol (mPEG-SH (5K)) from Fluka (The Netherlands) sodium borohydride $\left(\mathrm{NaBH}_{4}, 99 \%\right)$ from Merck (Germany) and ascorbic acid (99\%) from Aldrich (The Netherlands), OPSS-PEG-NHS (1600 MW) from Polypure (Denmark), RPMI1640 from Invitrogen (The Netherlands). All reagents were used as received. Prior to use, all glassware was cleaned with hydrofluoric acid (HF), further with aqua regia $\left(\mathrm{HCl} / \mathrm{HNO}_{3}\right)$ and rinsed twice with Milli Q water. 


\section{Synthesis of AuNRs}

Step 1: Synthesis of CTAB capped seed

A solution of CTAB $(9.75 \mathrm{ml} ; 0.1 \mathrm{M})$ was sonicated for 45 minutes at $40^{\circ} \mathrm{C}$ in a water bath. A solution of $\mathrm{HAuCl}_{4} .3 \mathrm{H}_{2} \mathrm{O}(0.25 \mathrm{ml} ; 0.01 \mathrm{M})$ was added with continuous stirring. Then, an ice-cold aqueous solution of $\mathrm{NaBH}_{4}(0.6 \mathrm{ml} ; 0.01 \mathrm{M})$ was added at once with vigorous stirring. This seed solution (CTAB-capped) is used during growth stage of nanorods as described in step 2 .

Step 2: Growth phase of synthesis

$9.5 \mathrm{ml}$ of a growth solution that consists of CTAB $(0.1 \mathrm{M}), 0.5 \mathrm{~mL}$ of $\mathrm{HAuCl}_{4} .3 \mathrm{H}_{2} \mathrm{O}(0.1 \mathrm{M})$ and $\mathrm{AgNO}_{3}(30 \mu 1$ of $0.006 \mathrm{M})$ was added resulting in an orange colored solution. Following this, the mild reducing agent ascorbic acid $(55 \mu 1$ of $0.1 \mathrm{M})$ was added to the orange colored growth solution that turned colourless. Finally, $12 \mu \mathrm{l}$ of a pre-formed CTAB-capped seed solution is added to the colourless solution. The solution was kept undisturbed for 24 hours for complete growth of nanorods. The gold nanorods were centrifuged once and the excess of unbound CTAB was removed. The nanorods were stored at $4^{\circ} \mathrm{C}$.

Further, the AuNRs were centrifuged (7000 g/20 minutes) and used for covalent bioconjugation with HER81 using a heterobifunctional PEG linker (OPSS-PEG-NHS). The PEG linker has a two functional groups at its ends- an N-hydroxysuccinimide group useful for coupling to amine group of HER81 and a -SS (disulfide) group that can couple to gold nanorod via thiol linkage. A schematic representation of this polymer is shown in Figure 1.

\section{PEG-Linker used for covalent bioconjugation of AuNRs}

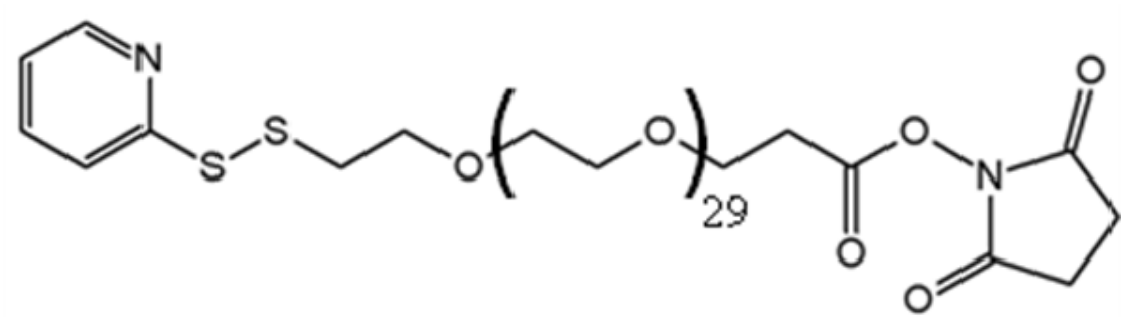

Figure 1: Heterobifunctional PEG linker (OPSS-PEG-NHS) used for covalent bioconjugation of gold nanorods. The PEG linker has an N-hydrosxy succinimidyl group at one end and a disulfide group at the other. 


\section{Bioconjugation of AuNRs via covalent interaction to HER81}

\section{Step 1: Coupling of OPSS-PEG-NHS (linker) to HER81 mAb}

The heterobifunctional PEG linker OPSS PEG NHS was used to couple the HER81 via amide bonding to the NHS group of linker and the -SS to couple to the AuNR. The linker is dispersed in $\mathrm{NaHCO}_{3}(100 \mathrm{mM}$, pH 8.5). $81 \mu \mathrm{mol} / \mathrm{L}(0.13 \mathrm{mg} / \mathrm{ml})$ OPSS-PEG-SH solution is mixed with $5.4 \mu \mathrm{mol} / \mathrm{L}(1 \mathrm{mg} / \mathrm{ml})$ HER81 $\mathrm{mAb}$ at a volumetric ratio of 1:9. The HER81OPSS-PEG-NHS was incubated on ice. The PEGylated antibody was dialyzed (MW=7,000) to remove excess of the linker. PBS buffer was used as dialysis buffer. The concentration of HER81-OPSS-PEG linker was measured using Nanodrop (Isogen Sciences). The samples were stored at $4{ }^{\circ} \mathrm{C}$.

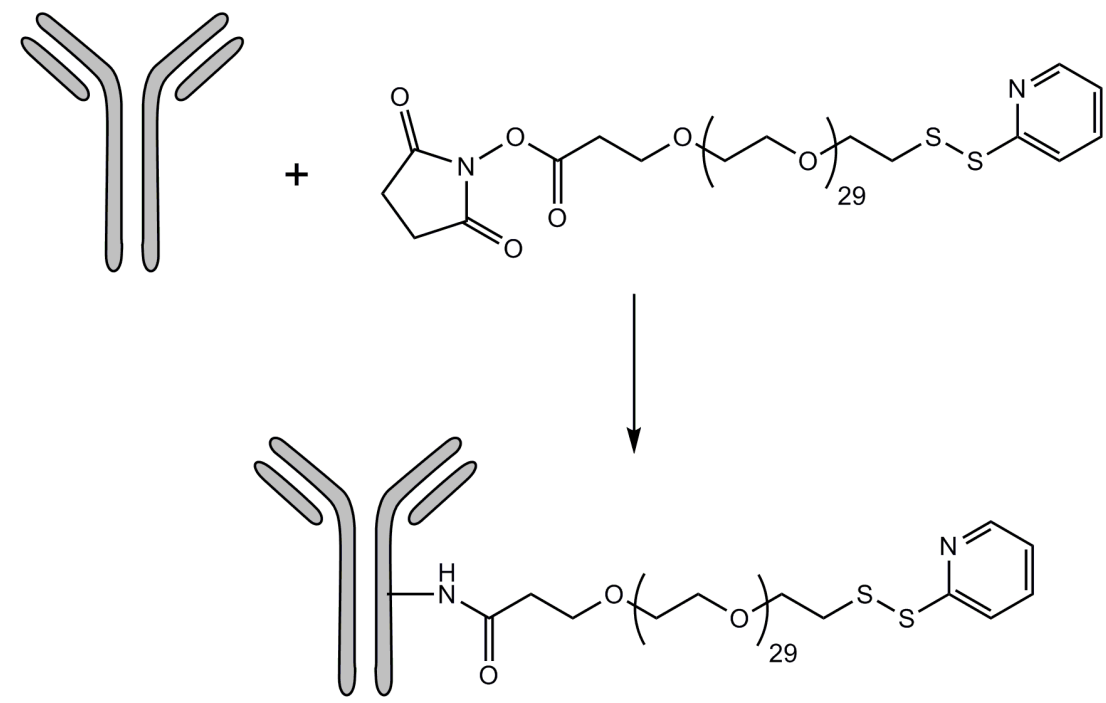

Figure 2: Schematic representation of lysine amino group of antibody coupling to NHS group of the PEG linker.

\section{Step 2: Coupling of PEG-linker-HER81 to gold nanorods via thiol linkage}

For a typical conjugation experiment, $2 \mathrm{ml}$ of gold nanorods are centrifuged at $7000 \mathrm{~g} / 20$ minutes in a low-binding micro tube. The supernatant is discarded and the pellets are added to increase the concentration of gold nanorods. The nanorods were later dispersed in $1 \mathrm{ml}$ PBS with $100 \mathrm{mM}$ EDTA and mixed gently. 


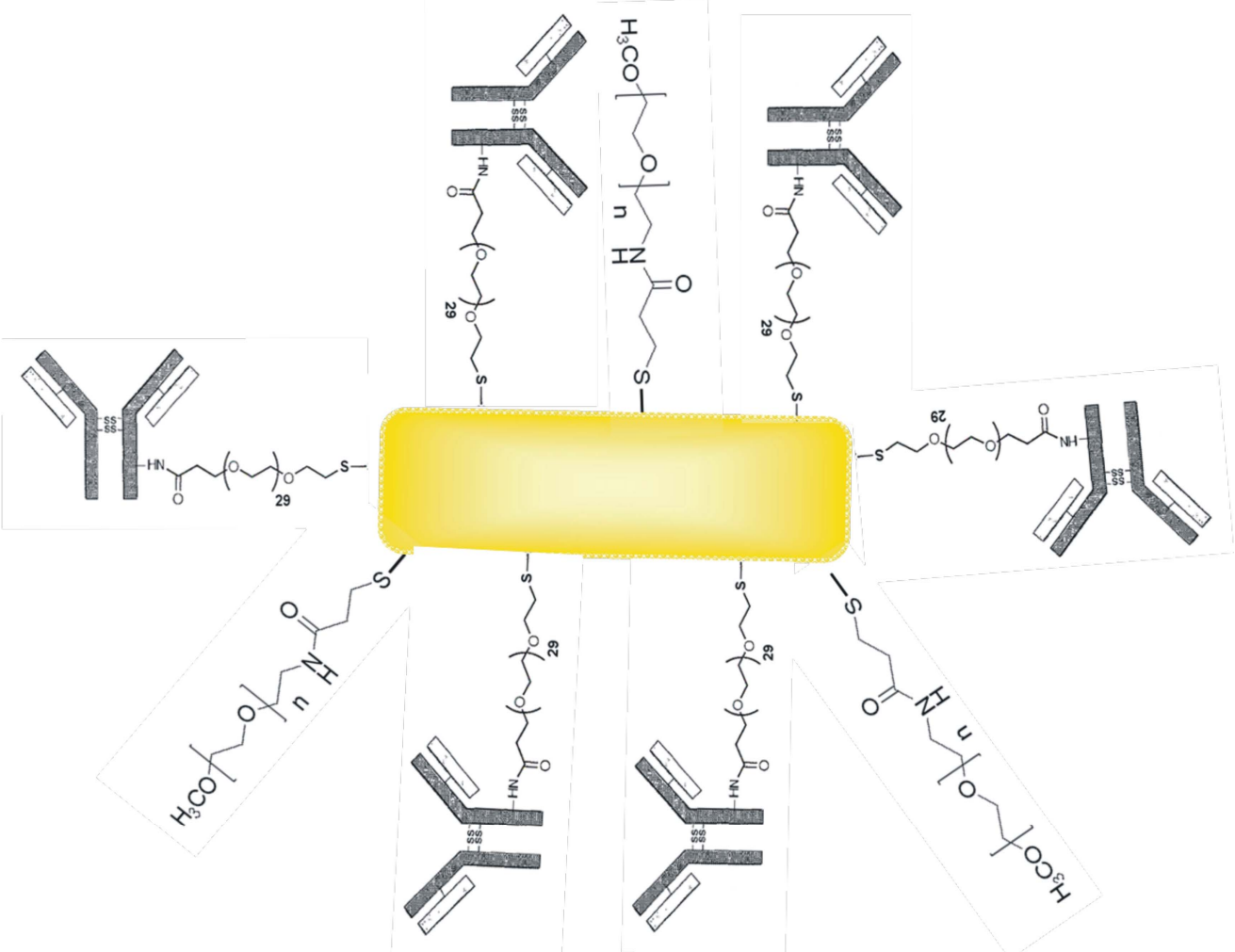

Figure 3: Schematic representation of gold nanorods binding covalently to PEG-linker-mAb complex via a thiol linkage. The free sites on the surface of nanorods are covered by using mPEG-SH.

Later, the PEG-linker-HER81 was added with vortexing and allowed to stand for two hours. After incubation, $100 \mu \mathrm{l}$ of $2 \mathrm{mM}$ potassium carbonate and $12 \mu \mathrm{l}$ of $5 \mathrm{mM}$ mPEG-SH was added. The sample was rotated overnight at room temperature (RT). Further, the sample was centrifuged three times at $7000 \mathrm{~g} / 20$ minutes and the supernatant was discarded. The pellet was dissolved in $1 \mathrm{X}$ PBS and stored at $4{ }^{\circ} \mathrm{C}$.

\section{Cell culture and cell-bioconjugate incubation with living cells}

The Her2/neu positive mammary adenocarcinoma (SKBR3) cell line was used as a HER81positive cell line. The cells were cultured in RPMI 1640 medium supplemented with $10 \%$ FBS (Fetal Bovine Serum) with antibiotics. Cells were maintained in an incubator at $37^{\circ} \mathrm{C}$ and $5 \% \mathrm{CO}_{2}$. The medium was changed every 3 days to keep confluent cells. Before the experiment, cells were harvested using a trypsin solution $(0.1 \%)$ and seeded onto $12 \mathrm{~mm}$ glass cover slips placed in a 6-well tissue culture plate, and allowed to grow for 2 days at $37^{\circ} \mathrm{C}, 5 \% \mathrm{CO}_{2}$ to reach $60 \%$ confluence. Cells were then washed with fresh PBS. Immunostaining was performed on cells and fixated in 4\% paraformaldehyde (PFA) for 20 minutes at room temperature. Silver staining using a silver-staining kit (Aurion, Wageningen, 
The Netherlands). was done on the cells with gold nanorod-antibody bioconjugate and visualization was performed using confocal microscopy.

\section{Electron microscopy on antibody conjugated AuNRs exposed to SKBR3 cells}

SKBR3 cells were exposed to conjugated gold nanorods were normalized to $62 \mathrm{pM}$ concentrations for $2 \frac{1}{2}$ hours. Exposed cells were fixed, in Karnovsky fixative. Post-fixation was done with $1 \%$ osmium tetroxide in $0.1 \mathrm{M}$ cacodylate buffer. After washing, the pellets were stained en bloc with Ultrastain-1 (Leica, Vienna, Austria), followed by an ethanol dehydration series. Finally, the cells were embedded in a mixture of DDSA/NMA/Embed812 (EMS, Hatfield, USA), sectioned and stained with lead oxide and analyzed with a CM10 electron microscope (FEI, Eindhoven, The Netherlands).

\section{Toxicity studies of antibody conjugated AuNRs on SKBR3 cells}

Here we have studied the toxicity of antibody conjugated gold nanorods when exposed to SKBR3 cells. For that purpose, $25 \mathrm{pM}$ of conjugated gold nanorods were incubated with SKBR3 cells for 48 hours. SKBR3 cells were placed on cover slips placed in a 24-well plate two days before the experiment and kept in an incubator $\left(37^{\circ} \mathrm{C}, 5 \% \mathrm{CO}_{2}\right)$ to reach $60 \%$ confluence. The nanorods solution were washed once in PBS and added to the culture medium of cells. After 4 hours incubation of the nanorods with the cells, the viability of the cells was assessed. Propidium iodide (PI) was added at a final concentration of $5 \mu \mathrm{g} / \mathrm{mL}$. After a few minutes incubation, cells were imaged using microscopy techniques and the amount of dead cells (i.e. red-stained nuclei) was qualitatively measured.

\section{Results}

\section{Optical spectroscopy of CTAB AuNRs and antibody conjugated AuNRs}

The optical peaks of gold nanorods are measured to determine the changes in the spectrum of as prepared gold nanorods after coupling with PEG-linker-antibody complex. The longitudinal plasmon peak of $\mathrm{AuNR}_{628 \mathrm{~nm}}$ did not shift as shown in figure 4, there was no broadening of the peak, and this is taken as evidence that the gold nanorods are stable after covalent linkage (thiol) with PEG linker antibody complex. The concentration of particles is lower due to loss of particles during the coupling of antibody-linker complex and during centrifugation steps. 


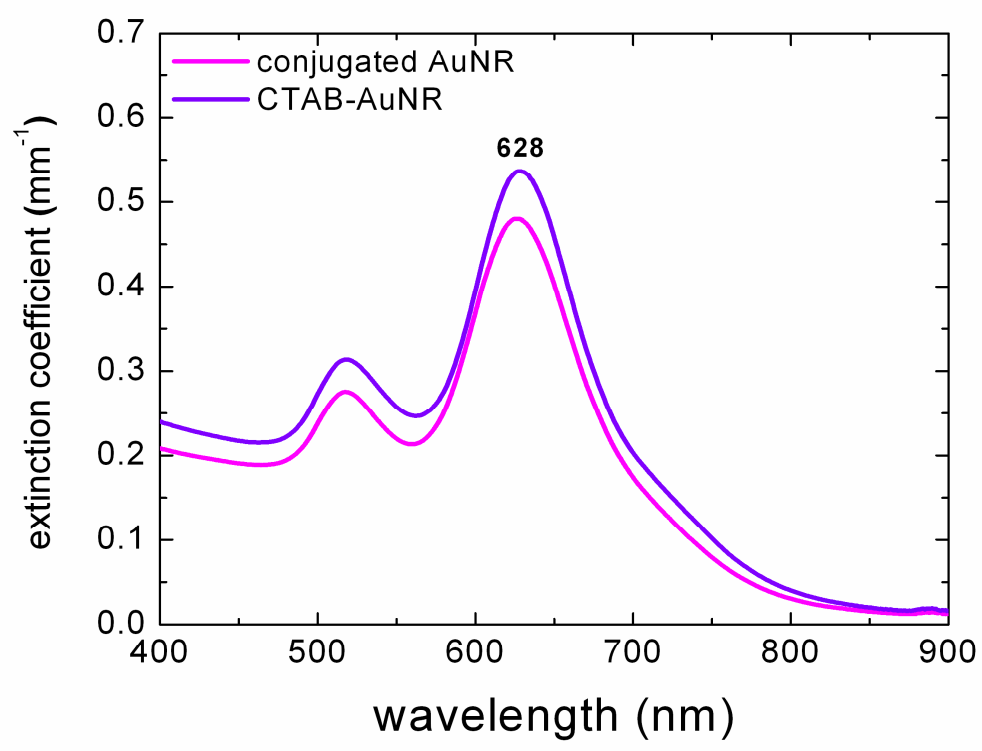

Figure 4: Optical spectroscopy of gold nanorods before/after bioconjugation with HER81 antibody via PEGlinker. The longitudinal plasmon peaks of gold nanorod before/after covalent coupling of antibody has a plasmon peak in the vicinity of $628 \mathrm{~nm}$. No aggregation was observed after coupling the PEG-linker-mAb complex to gold nanorods.

\section{Immunostaining of antibody conjugated AuNRs with SKBR3 cells}

The antibody conjugated gold nanorods are dispersed in media and are incubated with SKBR3 cells followed by fixation. Silver staining was done to enable visualization during confocal microscopy to study the fate of the gold nanorods in their interaction with the SKBR3 cells. In figure 5, the left panel is the confocal reflectance image and the right panel the bright field image of SKBR3 cells incubated with conjugated gold nanorods. The HER2 receptors are localized to the cell membranes of SKBR3 cells.

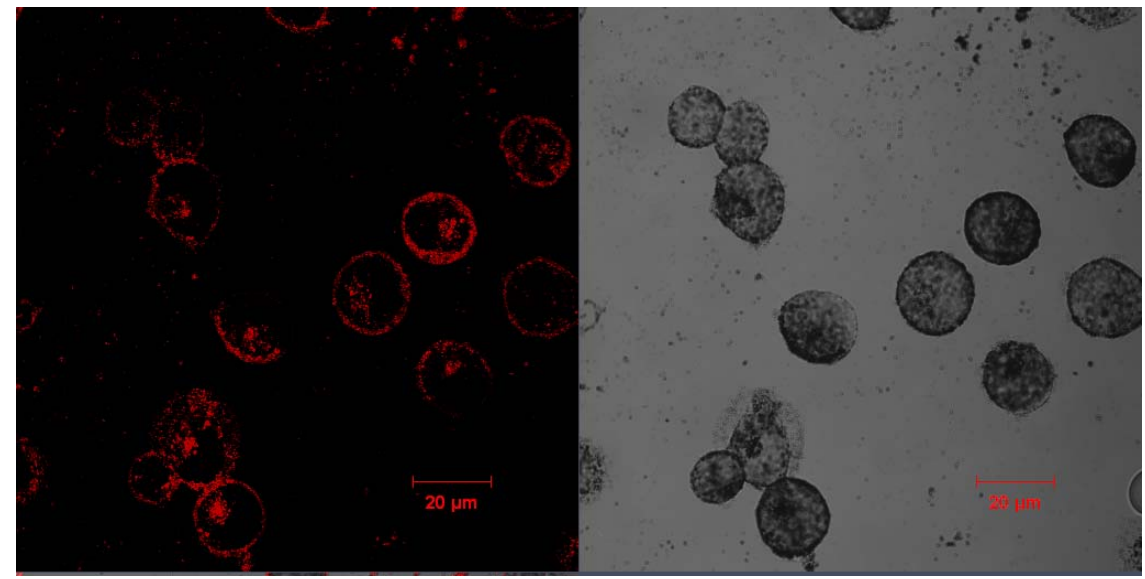

Figure 5: Immunostained confocal image of bioconjugated gold nanorods. Antibody conjugated AuNRs were incubated with SKBR3 cells for $2 \frac{1}{2}$ hours and later were fixated and silver stained. The right image is the reflectance of antibody conjugated gold nanorods and the left image is the bright field image. 
The high intensity of the signal at the cell membrane thus indicates collection of the antibody conjugated nanorods interacting with these receptors. This demonstrates successful conjugation and retention of functionality of the antibody after conjugation. There appears to be little or no internalization of the particles by the SKBR3 cells in the $2 \frac{1}{2}$ hours incubation time.

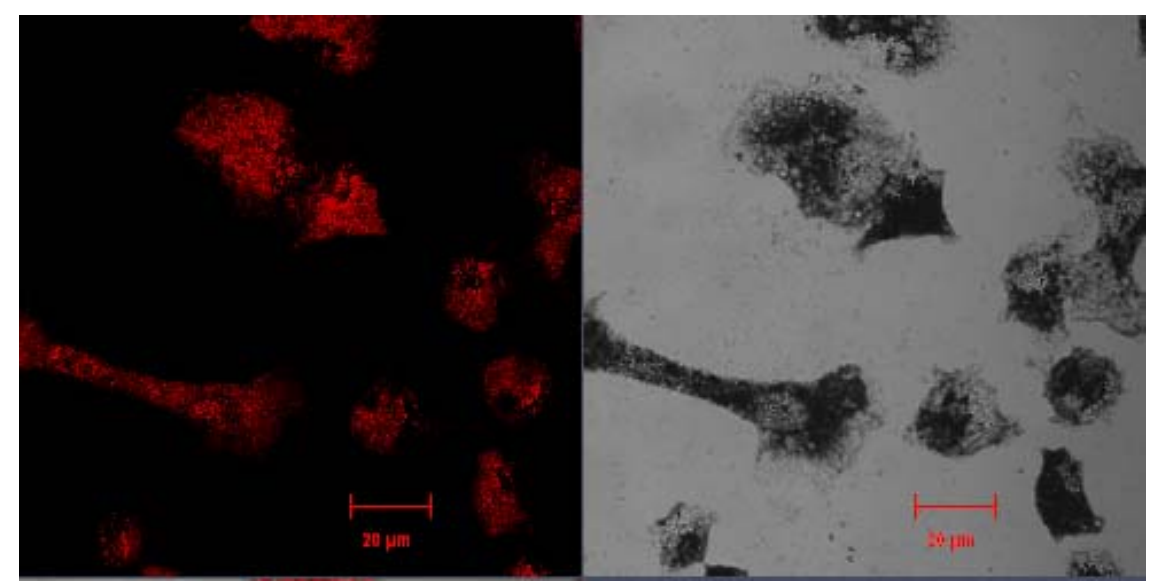

Figure 6: Immunostained confocal image of bioconjugated gold nanorods. Antibody conjugated AuNRs were incubated with SKBR3 cells for 48 hours and later were fixated and silver stained. The right image is the reflectance of silver-enhanced antibody conjugated gold nanorods and the left image is the bright field image.

\section{Negative controls}

The negative controls used in the study are the PEGylated gold nanorods (no antibody bound) incubated with SKBR3 cells and a Chinese Hamster Ovary (CHO) cell line that does not express EGFR receptors as shown in Figures 7 and 8.

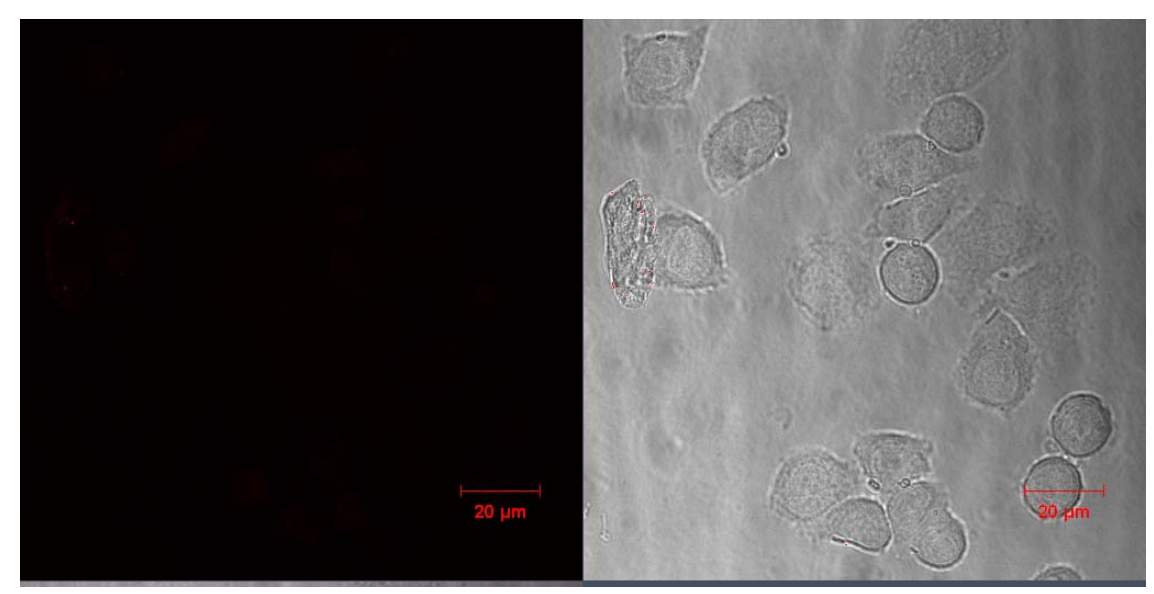

Figure 7: Immunostained confocal image of PEGylated gold nanorods with SKBR3 cells. The PEGylated gold nanorods were incubated with SKBR3 cells for 2 hours and later were fixated and silver stained. There is no reflectance of PEGylated gold nanorods on the cells in the left panel image. The right panel image is the bright field image of SKBR3 cells 


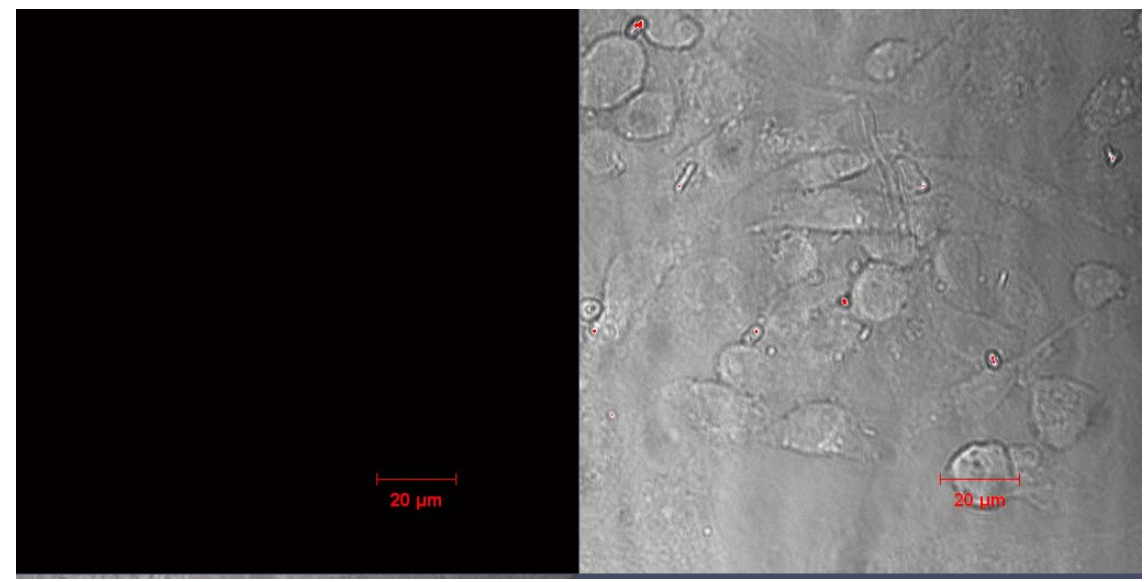

Figure 8: Immunostained confocal image of conjugated gold nanorods incubated with $\mathrm{CHO}$ cells. The $\mathrm{CHO}$ cells were incubated with antibody conjugated gold nanorods for 48 hours. There is no reflectance of antibody conjugated gold nanorods on the cell membrane indicating that the antibody conjugated gold nanorods have no interaction with $\mathrm{CHO}$ cell line. The right panel image is the bright field image of CHO cells.

There is no reflectance signal of gold nanorods when SKBR3 are incubated with PEGylated gold nanorods as shown in figure 7 . The $\mathrm{CHO}$ cells when incubated with antibody conjugated gold nanorods do not show any reflectance signal (Figure 8) as the antibody has no receptor to attach. Thus, the negative controls show no binding of gold to cells.

\section{TEM imaging of antibody conjugated gold nanorods interactions with SKBR3 cells}

The fate of the antibody-functionalized particles after an incubation time of $2 \frac{1}{2}$ hours with SKBR3 cells was studied using TEM. Figure 8(a) shows the particles interacting with the plasma membrane of a cell presumably binding to receptors. In figure 9(b), some nanorods are seen in endosomal vesicles, while in figure 9(c) particles are seen clearly in lysosomes. 

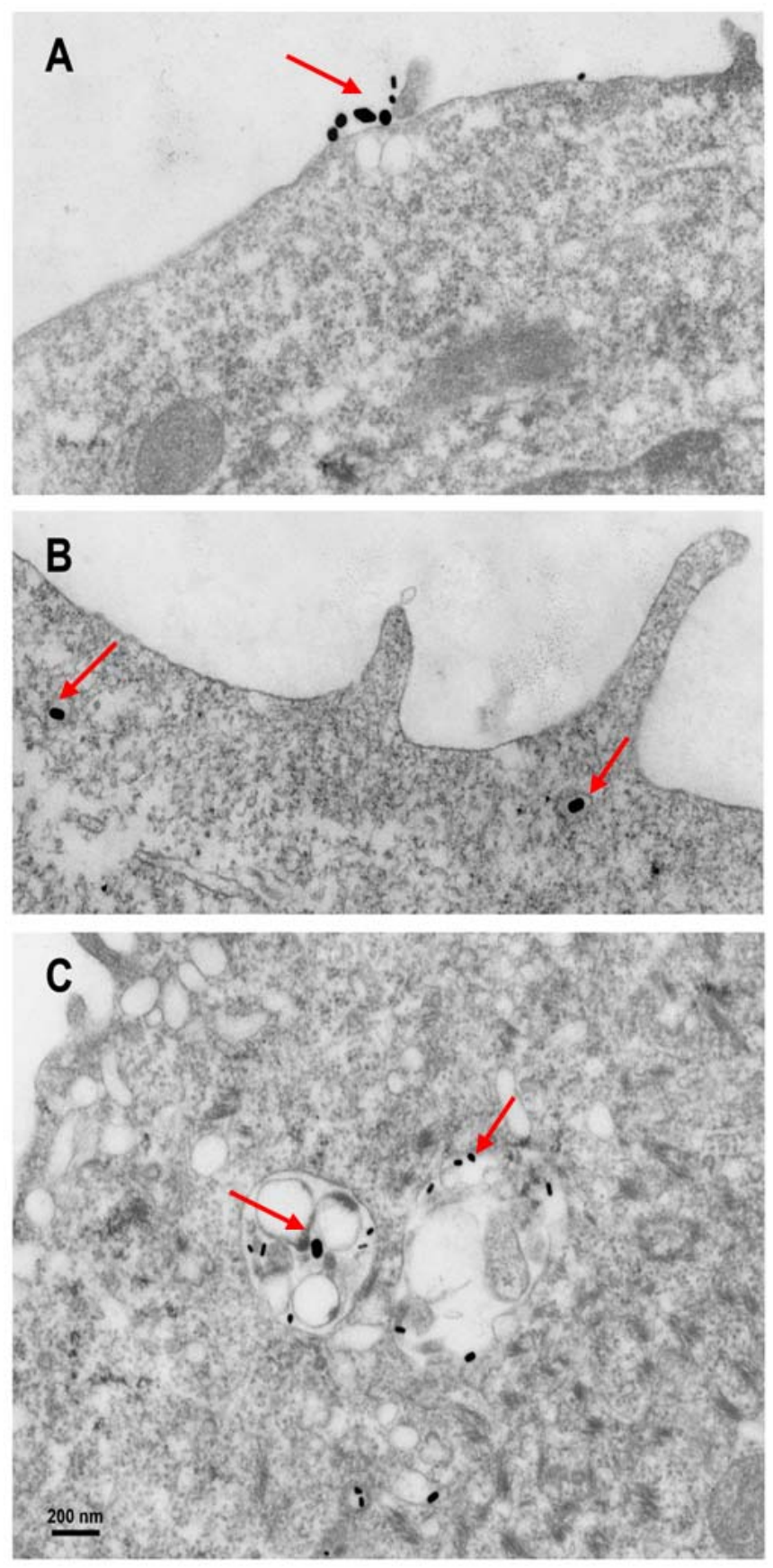

Figure 9: TEM images of antibody conjugated gold nanorods uptaken by SKBR3 cells in $2 \frac{1}{2}$ hours.

In the process of incorporation of foreign material, lysosomes produced in the cell travel to and fuse with the membranes of endosomal vesicles that carry these nanoparticles. The appearance of several particles in the lysosomes is not surprising since the organelle has the function to digest and recycle entrapped particles and does so by fusion with possibly several endosomes. 


\section{Toxicity of gold nanorods before/after conjugating antibodies to gold nanorods}

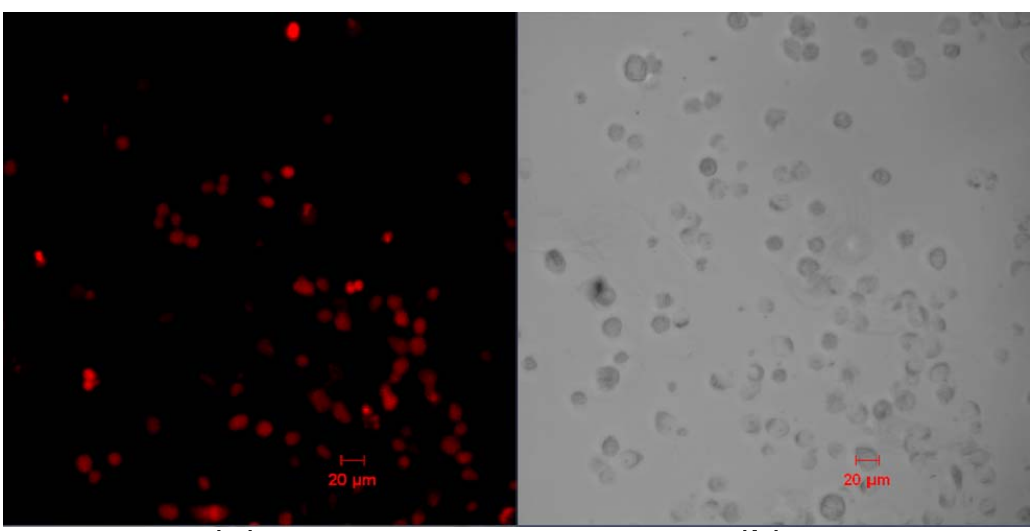

(a)

(b)

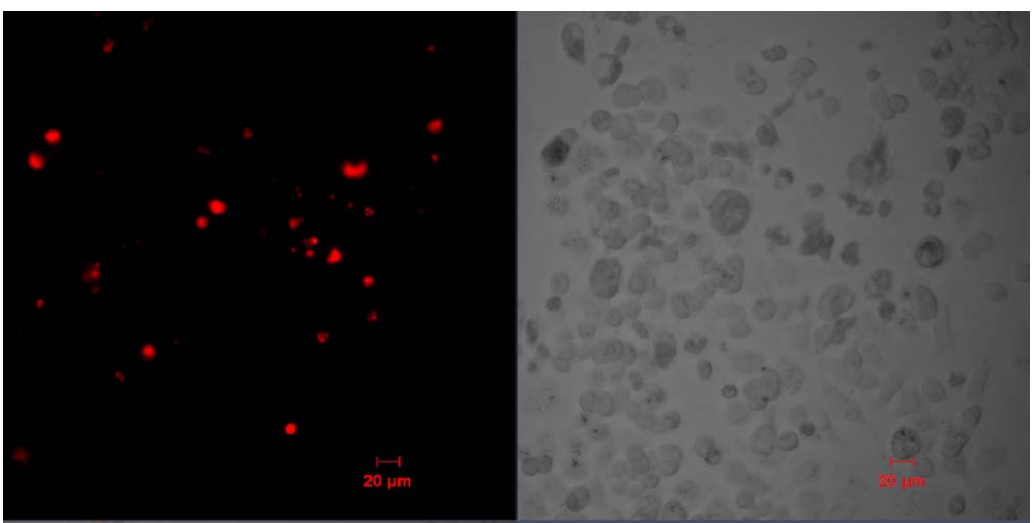

(c)

(d)

Figure 10: Confocal images of gold nanorods before/after antibody coupling to SKBR3 cells. The toxicity is evaluated using Propidium iodide assay. The as prepared CTAB capped gold nanorods are toxic to SKBR3 cells (figure 10(a)) and the dead cells nuclei are stained red. The antibody conjugated gold nanorods incubated with SKBR3 cells is not that toxic as shown in figure 10(c). The bright field images of SKBR3 cells are shown in $10(\mathrm{~b})$ and (d).

The as prepared and antibody conjugated gold nanorods were incubated for 48 hours with SKBR3 cells. The cell viability of antibody conjugated AuNRs was assessed using a nuclear staining (PI or propidium Iodide) assay. When there is cell death, the cellular membrane becomes permeable so that cells take up PI, and the nucleus becomes stained red. In figure 9, the left panels (figures 10 (a) and (c)) shows the nucleus red-stained indicating cell death. The right panels (figures 10 (b) and (d)) represents the bright field images that show the morphology of the cells. The fluorescence image shows few red-stained nuclei (i.e. dead cells); we do not observe significant cell death for 48 hours of the cell exposure to the conjugated gold nanoparticles in comparison to as-prepared gold nanorods. 


\section{Discussion}

Nanoparticles with applications in biomedicine, such as improving specificity for diagnostic imaging and therapy are necessarily functionalized to have affinity for the disease state and site. If the entry of the particles into the body is by systemic administration via an injection into a vein, the particles require to be colloidally stable in the presence of biomolecules such as serum proteins which abound in the microenvironment of blood. The particles should also have their reactive surfaces coated with biocompatible materials which makes the nanocomposites less toxic and imparts stealth characteristics to them. This would help them to evade phagocytic attack and incorporation into cells of the reticuloendothelial system (RES) $[6,7]$.

To achieve these features in our gold nanorods to make them suitable for in vivo applications, we have functionalized them with the HER81 antibody which recognizes HER2, the receptor that is overexpressed in $20-40 \%$ of human breast cancers. The mode of conjugation was covalent coupling that is expected to be more stable and stronger than the common electrostatic-hydrophobic binding discussed in Chapter 2. Specifically a heterobifunctional PEG linker was used, with an NHS group at one end for coupling to the amine group of the antibody and a disulfide group at the other end that can couple to gold nanorod by strong thiol binding. PEG is chosen to be the spacer due to its hydrophilic and biocompatible properties. Further, the nanocomposite is treated with mPEG-SH to passivate any exposed surfaces.

PEG has stealth properties and can evade the RES response, thereby improving circulation time of functionalized gold nanorods. This prolonged circulation time in blood improves opportunities for the particles to arrive at the target cells.

The optical imaging studies of fixated SKBR3 cells incubated for $2 \frac{1}{2}$ hours with the antibody coupled nanorods using confocal and bright field microscopy (figure 5) successfully detected the bioconjugates. The particles are seen to congregate at the membranes of the HER2 positive cell line where the receptors are expressed, indicating the success of the conjugation procedure. Longer incubation times of 48 hours showed massive uptake of the nanorods (figure 6). This shows that the cellular uptake of conjugated gold nanorods can be controlled by their incubation times. The internalization of the particles did not appear to elicit a toxic response and the SKBR3 cells remained intact and viable as seen in figures 5 and 6 . To verify 
this, cell viability studies were conducted for the two incubation times using a PI staining assay. No significant toxicity was observed in the cells for $2 \frac{1}{2}$ hours and 48 hours incubation (figure 8).

To elucidate the mechanisms of cellular uptake and the fate of the particles in the cells, we performed TEM imaging of the cells exposed to the particles for $2 \frac{1}{2} 2$ hours. Our selection of the images of cells in figure 7, capture the various stages in the pathway of nanoparticulate cellular incorporation and intracellular transport. The route starts with the binding of the antibody to the receptors on the plasma membrane a process that is suggested by figure 7(a). The actual internalization via invagination of the plasma membrane has not been captured, but figure 7(b) shows particles trapped in membrane limited (nanovesicles) [13], which are endosomes. The next image (figure 7(c)) shows a considerable number of particles in lysosomes, with which in all probability endosomes have fused transferring the foreign particles to these organelles. It is expected that the foreign particulate matter is processed in lysosomes using acidic enzymes, before the resultants are transported to the cell periphery for excretion. Thus, even though the imaging was not a time evolution study of uptake and transport behavior since a constant incubation time was applied, nevertheless we have captured different cells at various stages of the endo-lysosomal pathway starting with receptor-mediated endocytosis (RME). The cellular morphology was intact despite the uptake of the gold nanorods by the cells as seen in figure 7 . Taken together with the viability studies above these results show that the HER81-AuNRs are non-toxic for the conditions studied.

It should be mentioned that even though cells may appear normal, their function and growth could be impaired or that they may be genetically altered, which could have consequences in the end. We will examine these aspects in detail in future studies. From an application point of view, it is important to ascertain the average numbers of antibody conjugated nanorods, which can attach to or be taken up by the cells. This will provide an estimation of the contrast enhancement effect or therapeutic effect that can be elicited from such "marked" cells. We will study this issue using analysis techniques such as inductively coupled plasma mass spectrometry (ICP-MS).

Ultimately, we wish to study the molecular probes developed in vivo for the efficacy of targeting via biodistribution investigations in small animals. These studies should present interesting results, as the particles will be challenged by the complexity of blood flow, immunological and inflammatory responses [14]. 


\section{Conclusions}

We have synthesized gold nanorods with optical extinction peaks in the region from 600 $850 \mathrm{~nm}$ making these eminently suited for scattering and absorption contrast enhancements in optical imaging. We covalently conjugated the gold nanorods using heterobifunctional PEG linker with HER81 antibodies which have affinities for receptors expressed by SKBR3 breast carcinoma cells. The binding of the PEG-linker to the gold surface is achieved via strong covalent thiol coupling. The conjugated gold nanorods are colloidally stable and the antibodies remain biologically active. There was massive uptake of antibody conjugated gold nanorods by SKBR3 cells with 48 hours exposure observed using confocal microscopy. The TEM analysis confirmed that the uptake mechanism is receptor mediated endocytosis and follows an endo-lysosomal pathway. The TEM images show snapshots of various stages of the cellular uptake and intracellular transport. In spite of the incorporation of the nanoparticles, the cells showed minimal toxic responses. Future studies will focus on studying the behavior and distribution of these conjugated particles in vivo. These findings have potential for in vivo biomedical applications.

\section{Acknowledgements}

The HER81 antibody and SKBR3 cells were received from Prof. Leon Terstappen. Transmission Electron Microscopy was carried out by Patrick Chin, Hans Janssen and Dr. Fijs van Leeuwen (NKI). The research is funded by the University of Twente through the thrust area program NIMTIK; and by the Nederlandse Wetenschappelijk Organisatie (NWO) and Stichting Technische Wetenschappen (STW) through project TTF 6527.

\section{References}

1. P.K. Jain, K. Seok Lee, I.H. El-Sayed and M.A. El-Sayed, J. Phys. Chem. B, 110, pp. 7238-7248, 2006.

2. I.H. El-Sayed, X. Huang and M.A. El-Sayed, Nano Lett., 5, pp. 829-834, 2005.

3. J.A. Copland, M.A. Eghtedari, V.L. Popov, N. Kotov, M. Motamedi and A. Oraevsky, Mol Imaging Biol, 6, pp. 341-349, 2004.

4. X. Huang, I.H. El-Sayed and M.A. El-Sayed, J Am. Chem. Soc, 125, pp. 1215-1220, 2006.

5. R.G. Rayavarapu, W. Petersen, C. Ungureanu, J.N. Post, T.G. van Leeuwen and S. Manohar, Intl.J. Biomed.Imaging, 29817, 2007.

6. T. Niidome, M. Yamagata, Y. Okamoto, Y. Akiyama, H. Takahashi, T. Kawano, Y. Katayama and Y. Niidome, J. Control Release, 114, pp. 343-347, 2006.

7. R.G. Rayavarapu, W. Petersen, P. Chin, H. Jansen, F. van Leeuwen, S. Manohar and T.G. van Leeuwen, Nanotechnology, (in print), 2010.

8. R.G. Rayavarapu, W. Petersen, S. Le Gac, C. Ungureanu, T.G. van Leeuwen and S. Manohar, SPIE proceedings, 2007.

9. H. Liao and J. Hafner, Chem. Mater, 17, pp. 4636-4641, 2005. 
10. C. Loo, A. Lin, L. Hirsch, M.H. Lee, J. Barton, N. Halas, J. West and R. Drezek, Technol. Cancer Res. Treat. 3, pp. 33-40, 2004.

11. T.B. Huff, M.N. Hansen, Y. Zhao, J. Cheng and A. Wei, Langmuir, 23, pp. 1596-1599, 2007.

12. M. Eghtedari, A.V. Liopo, J.A. Copland, A. Oraevsky and M. Motamedi, Nano Lett., 9, pp. 287-291, 2009.

13. B.D. Chithrani, J. Stewart, C. Allen and D.A. Jaffray, Nanomedicine, 5, pp. 118-127, 2009.

14. B.D. Chithrani and W.C.W. Chan, Nano Lett., 7, pp. 1542-1550, 2007. 


\section{Chapter 8}

\section{Conclusions and Outlook/Recommendations}

In this thesis, we describe the development of functionalized gold nanorods as molecular probes that can be used in vivo for light-based imaging and treatment techniques. Therefore, these functionalized gold nanoparticles should be biocompatible, stable, with the ability to target tumor cells and consequently can be used in vivo for light-based imaging and treatment technique. Due to strong light absorbance by hemoglobin and other tissue chromophores in the blue and green part of the spectrum, the latter requirement determines that the gold nanorods should have their peak absorbance in the near infrared region (NIR- $600 \mathrm{~nm}-950$ $\mathrm{nm}$ ), in the so called the optical therapeutic and imaging window [1, 2]. At these wavelengths, the gold nanorods can be used as contrast agents/molecular probes that help in improving the specificity and sensitivity of multiple imaging modalities such as optical coherence tomography, two photon luminescence and photoacoustic imaging [3]. 
As a first proof of principle, gold nanorods (AuNRs) were synthesized in high yield using silver-assisted surfactant method having extinction peaks in the region from $600 \mathrm{~nm}-900$ $\mathrm{nm}$, making them eminently suited for absorption contrast enhancers in optical imaging. To provide targeting ability for a certain disease state such as cancer, we functionalized with HER81 antibodies via non-covalent (electrostatic/hydrophobic) binding [2]. In Chapter 2, it was shown that, the HER81 monoclonal antibodies have affinities for the HER2/neu receptors expressed by SKBR3 breast carcinoma cell line. However, these experiments were performed in vitro on fixed cells, which is clearly a different situation than the complex in vivo conditions.

$\mathrm{CTAB}$ acts as a shape-directing agent $[4,5,6,7]$ to synthesize the gold nanorods by passivating the $\mathrm{Au}\{110\}$ facets of the seed so that gold deposition takes place at facets which ultimately make the tips of the rod. Recently it was reported by Korgel et al [8] that certain supplier products of CTAB did not induce nanorods formation but formed only nanospheres, which was attributed to the presence of iodide impurities in CTAB powders, as determined from ICP-MS measurements. In Chapter 3, we presented our investigation using ICP-MS analysis that showed that our CTAB powders had no detectable iodide that obstructed the formation of nanorods. This was seen for the same CTAB products as identified by a supplier and product number. However, by forced contamination of the CTAB by potassium iodide (KI) we found that only nanospheres were formed instead of nanorods. This shows that CTAB supplier products can vary in impurity from lot to lot as shown in the results, which can have a dramatic influence on the reproducibility of the GNR production.

The effect of the chemical procedure was further investigated in chapter 4 . Whereas conventional protocols to synthesize cylindrical gold nanorods use low volumes of ascorbic acid (AA) in the growth solution [1, 2, 7, 8], we showed that by increasing the volume of AA in the growth solution, the shape of the normal hemispherically-capped cylinder (nanorods) changes. The nanorod, dog-bone, dumbbell and finally indeterminate forms are seen as the ratios of ascorbate to gold ions are varied from the standard 1.1:1 to $2: 1,3: 1$ and $4: 1$. We presented models to explain the formation of these shapes during particle formation. The changes in shape of nanorods to other morphologies also showed optical changes like blueshifting of the longitudinal plasmon peaks which we satisfactorily explained.

For use of gold nanorods and other nanostructures in in vivo biomedical applications, the nanorods should be stable and biocompatible $[9,10,11]$. Hence, in Chapter 5 we studied 
CTAB capped and polymer (PEG, PSS) treated gold nanorods on cell viability in different cell lines. The as-synthesized CTAB capped gold nanorods are toxic to all studied cell lines even at low picoMolar (pM) concentrations. To mitigate toxicity, we coated the surfaces of gold nanorods with thiolated-PEG and $\mathrm{Na}$ salt of polystyrene sulfonate (PSS). PEGylation helped in displacing the CTAB bilayer from the surface of nanorods, and PSS polymer was bound electrostatically to CTAB bilayer thereby encapsulating it. The covalent coupling of PEG to gold nanorod via thiol $(\mathrm{SH})$ makes the nanorods stable and no aggregation is observed. PEGylated gold nanorods are also found to be stable in presence of cell medium containing various serum proteins. Our results showed that the use of coating gold nanorods with PSS and possibly with other polyelectrolytes (PAA, PAH) is not the best approach to remove toxicity of CTAB capped gold nanorods.

Treatment with PSS and possibly other polyelectrolytes, is an inferior detoxification approach since the polymer only encapsulates the AuNR with its CTAB bilayer intact. PSS-AuNRs cluster and coagulate on exposure to cell culture medium (shown in chapter 5), which will further hamper future use in vivo. TEM analysis of the uptake of nanorods when incubated with SKBR3 cells showed that the PEGylated gold nanorods appear not to interact with cells and no uptake was observed. In case of PSS coated gold nanorods, there is uptake by cells via non-specific endocytosis. This in vitro study is a quick and relatively inexpensive method of testing surface modifications of nanorods to improve their biocompatibility. Furthermore, this study showed that the PEGylated gold nanorods are favorable for in vivo use.

The CTAB capped gold nanorods and PEGylated gold nanorods were tested for their fate in vivo. In 18 rats, we studied the biodistribution of gold nanorods at two and seven days after intravenous injection in blood and several organs (liver, spleen, kidney, lung, heart, thymus, brain and testes). The circulation time of gold nanorods in blood is an important aspect for therapy/clinical applications if the particles are to be used in targeting of disease in vivo. The non-PEGylated CTAB capped gold nanorods were sequestered to the liver mainly, and to a lesser extent to the spleen followed by the lung after administration into the rat. The PEGylated gold nanorods were found in the blood even after 48 hours after administration. In addition, the accumulation of CTAB capped gold nanorods appears to be higher in the liver, whereas the PEGylated gold nanorods showed an increased accumulation in the spleen. Another important finding is that no accumulation of gold nanorods was observed in the brain 
tissue. PEGylated gold nanorods appear to be promising candidates to be used for in vivo applications as their retention time in blood circulation is longer and aid in imaging during therapeutic applications.

For using gold nanorods as molecular probes for targeting pathologies in vivo, we have performed covalent bioconjugation of gold nanorods using a hetero functional PEG-linker that has a disulfide and -NHS moieties at either end. The disulfide reacts covalently with Au nanorod and the NHS moiety binds to amine group of HER 81 via peptide $\left(\mathrm{CO}-\mathrm{NH}_{2}\right)$ bonding. When these conjugated gold nanorods are incubated for 48 hours with SKBR3 cells, we observed a major uptake of conjugated gold nanorods by these cells. Such a trend was not observed with lower incubation of conjugated gold nanorods with cells. These results show that cellular uptake is increased with incubation time of particles with cells. Qualitative toxicity studies using a propidium iodide assay, and by microscopy, showed that the cells looked intact and healthy and were viable. Thus, the conjugated gold nanorods are biocompatible when exposed to cells and were successfully targeted to receptors of tumor cells that have potential to be used as molecular probes for clinical applications.

\section{Outlook/Future recommendations for improving gold nanorods as contrast agents}

As described in Chapter 2, the gold nanorods are synthesized using wet-chemical seed mediated method in final volumes of $10 \mathrm{ml}$ batches. Aliquots of such samples of gold nanorods based on CTAB stabilized Au seeds are shown in Figure 1,

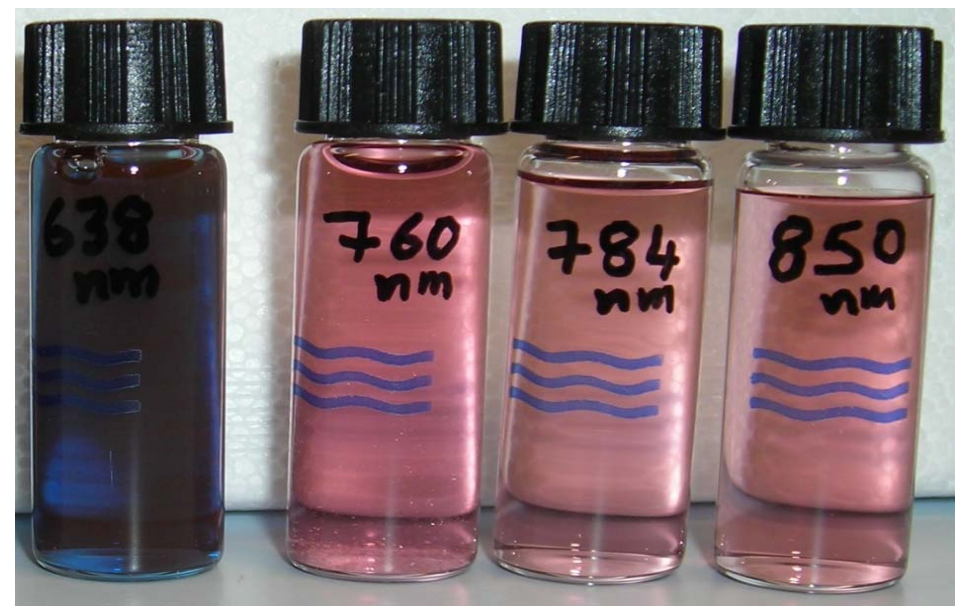

Figure 1: Gold nanorod solutions of $10 \mathrm{ml}$ final volume of batches spanning along the far visible up to near infrared (NIR) region of spectrum. The batches of gold nanorods (from left to right) have longitudinal plasmon peaks of $628 \mathrm{~nm}, 760 \mathrm{~nm}, 784 \mathrm{~nm}$ and $850 \mathrm{~nm}$ respectively. 
For practical purposes, gold nanorods should be synthesized in high yield and in larger quantities, e.g. to facilitate comparative studies based on the same batch of nanorods. Furthermore, the scaled-up synthesis of nanorods is required because of losses (concentration of nanorods) occurring during various processing steps such as coating of nanorod surfaces by polymers (PEGylation or PSS coating as shown in chapter 5). Next, during conjugation of gold nanorods with targeting antibodies, particles will also be lost. In both these cases, the bulk of losses occur during centrifugation steps to concentrate or purify samples. Finally, for large scale studies of biocompatibility and biodistribution (as in chapters 5 and 6), large quantities of high-concentrations samples will be required.

Scaled-up synthesis in bulk volumes was done as shown in Figure 2. The $100 \mathrm{ml}$ scaled up batch (figure $2 \mathrm{a}$ ) has a concentration of $3.5 \times 10^{10} \mathrm{NRs} / \mathrm{ml}$ and the liter solution (figure $2 \mathrm{~b}$ ) has a concentration of $1.2 \times 10^{11} \mathrm{NRs} / \mathrm{ml}$. However, the scaling up of synthesizing nanorods is a laborious process (e.g. pipetting/centrifugation). To resolve this, one has to design automated machinery without manual use that would speed up the synthesis and reduce batch-to-batch variation.
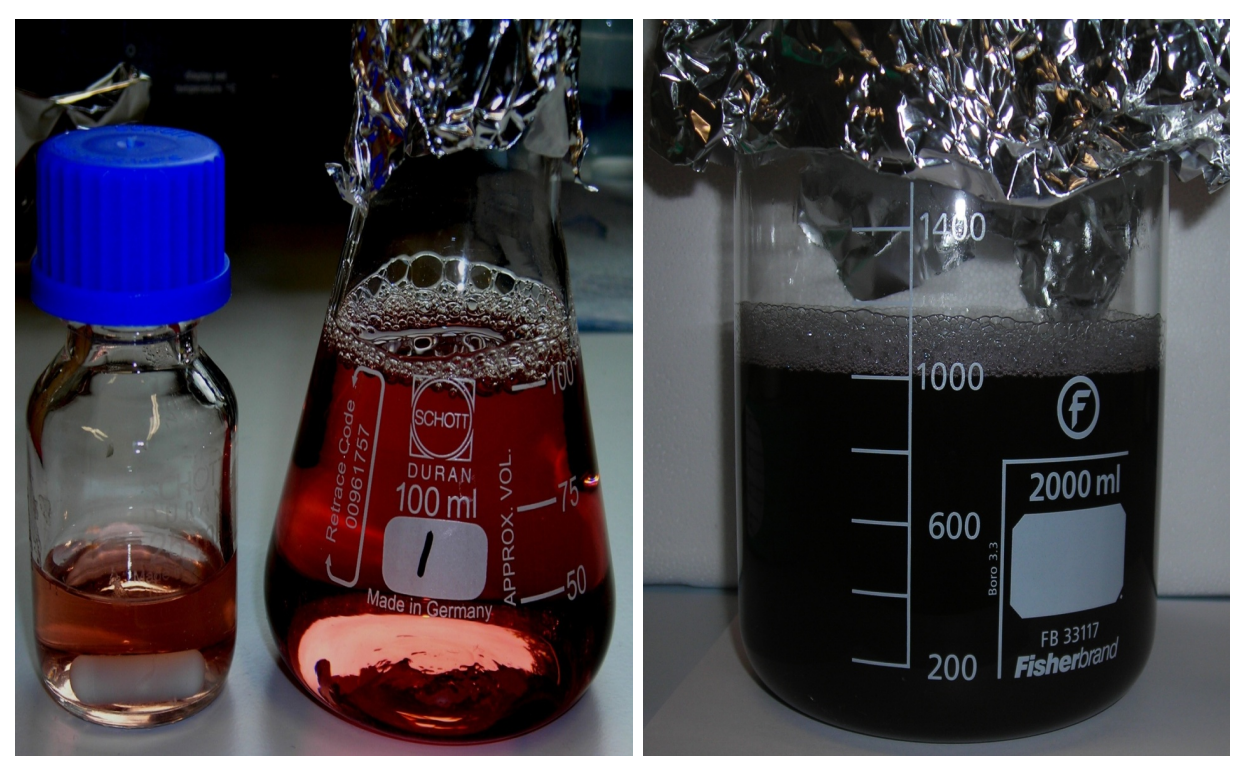

Figure 2: An attempt to scale up synthesis of gold nanorods. Figures 2 (a), (b) shows pre-formed CTABstabilized $\mathrm{Au}$ seeds and respective solutions of nanorods synthesized in volumes of $100 \mathrm{ml}$ up to 1 liter.

In chapter 2, we have mentioned that $\mathrm{CTAB}$ act as a stabilizer and shape-directing agent for synthesizing gold nanorods. However, CTAB is toxic to cells (Chapter 5) and causes a hindrance for use in in vivo applications [9, 10]. Thus, gold nanorods synthesized without CTAB or low concentrations of CTAB would be favorable. Such an optimization of the synthesis procedure could for example be achieved by post-treatment of CTAB capped gold 
nanorods. The excess CTAB can be extracted from a NR solution into a chloroform phase containing phosphatidylcholine (PC). Very negligible amount of CTAB is present after the process of extraction using PC-chloroform [11]. This is a better alternative in removing excess of $\mathrm{CTAB}$ compared to filtration or dialysis where there is loss of particles. The gold nanorods coated with mPEG-SH does not allow further for functionalization of organic molecules or proteins. In case of PC-modified gold nanorods, the phosphotidylcholine (phospholipids) can be attached to functional groups that allow functionalization of gold nanorods [12].

PEGylated gold nanorods have minimal interaction with cells and do not demonstrate major uptake into these cells. For some applications such as photo thermal therapy applications, this behavior may be disadvantageous. The cellular uptake of conjugated gold nanorods could be necessary for photothermal therapy where there can be local confinement of heat and thus tumor killing can take place without affecting the healthy cells. Hence, the gold nanorods conjugated using PEG-linker attractive because there is uptake of conjugated gold nanorods via receptor-mediated endocytosis.

\section{Towards clinical applications of AuNRs- for molecular imaging and therapeutic techniques}

New patient screening methods, especially based on new genomics and proteomics techniques will increase the number of patients known to be susceptible for having tissue metaplasia and dysplasia. Two potential problems arise: first, these small malformations have to be detected in vivo by non-invasive and non-ionizing imaging techniques, and, secondly, these malformations have to be eradicated. The detection of the small malformations depends on the sensitivity of the currently used imaging techniques, like ultrasound, photo-acoustic imaging and optical (coherence, fluorescence) tomography and digital signal analysis of MRI and CT images. The contrast of these imaging techniques, based on differences in fluorescence, scattering and absorption between target tissue and 'non-pathological' tissue, however, is either too low or unspecific to allow early detection of tumors. Therefore, identification of tissue pathologies on a molecular level by using specific antibodies coupled to biocompatible contrast enhancers is being pursued.

The large absorption efficiency of gold nanorods in the NIR part of the spectrum in combination with successful bioconjugation, may facilitate both early detection an 
eradication of tumors. The absorption of pulsed light (with pulse duration in the order of ns) will induce pressure waves, which after subsequent detection and reconstruction can be used to image the tumors. This method called photoacoustic imaging [13-16] can then be used for screening of suspected people. In the case of detected pathologic tissue, the non-invasive eradication of tissues by hyperthermia is a logical next step. Hyperthermia, the local heating of tissue, by the absorbed laser energy, is based on the higher sensitivity of tumor cells compared to normal cells for eradication by local heating. Although some chemicals aspects in the production process can still be optimized, we demonstrated that functionalized gold nanorods can be reproducibly made, are biocompatible, stable, with the ability to target tumor cells and consequently can be used in vivo for light-based imaging and treatment technique.

Most of the work reported in Chapter 7, the bioconjugation using covalent binding of PEGlinker (OPSS-PEG-NHS) to gold nanorods are silver stained and visualized using confocal microscopy and Scanning Electron Microscopy. The drawback of silver staining is that it can create artifacts where silver enhancement is not from a single gold nanorod but a cluster/aggregate of gold nanorods on the cell membrane or interior. To avoid such an artifact, the gold nanorods should be coupled with a fluorescent dye that gives good resolution and track the fate of gold nanorods inside the cell. In addition, fluorescently labeled gold nanorods can be visualized using Atomic Force Fluorescence microscopy (AFFM). This is a relatively inexpensive method compared to Transmission Electron Microscopy (TEM) that is laborious in sample preparation. The cells and the concentrations of particles should be high during sample preparation for TEM studies.

Gold nanorods can be attached to antibody coupled fluorescent dyes (antibody-FITC) via the FITC's isothiocyanate group that has affinity to gold. Thus, silver staining can be avoided and the presence of gold nanorods on the cell or within the cell can be tracked using fluorescence microscopy. Another approach is to use Ellman's reagent, a chemical that can be used to quantify the number of thiols [17]. This can determine the number of thiols on PEG linker bound antibody giving an estimation of the number of linkers attached to an antibody-AuNR complex and further the number of conjugated gold nanorods on cells.

Next, an appropriate ligand for functionalization of the gold nanorod has to be chosen. In Chapters 2 and 7, we have used HER81 monoclonal antibody coupling them via non-covalent and covalent interaction with receptors of breast carcinoma cell-line. However, antibodies are 
not always stable, and denature at higher temperature (e.g. during laser irradiation). The alternative is to use aptamers that are more stable at higher temperatures. Aptamers bind strongly to human IgE (immunogloblulin E), and compared to antibodies, have longer shelf life and can be synthesized chemically in large quantities [18]. Another important aspect is that aptamers, are stable at ambient temperatures and when denatured can be made active or regenerated within minutes. Apart from aptamers, nanobodies are also advantageous compared to antibodies. Nanobodies have resistance to heat and $\mathrm{pH}$ unlike antibodies. These are also used for targeting colon and gut tumors in vivo [19].

The biodistribution of gold nanorods should be further studied in small animals that are bearing tumors. It may well be, that PEGylated gold nanorods may extravasate into solid tumor tissue through leaks in the vasculature [20]. The additional effect of the bioconjugation on the uptake into the tumor and the consequent increase in contrast for imaging and the effect on tumor death upon laser-induced hyperthermia has to be studied.

The results obtained in this thesis using gold nanorods shows the potential for the developed particles to be successful these applications in vivo. The functionalized gold nanorods with tunable optical properties can play a vital role in molecular imaging modalities that allow merging of the diagnostic and therapeutic technologies.

\section{References}

1) B. Nikoobakht and M.A. El-Sayed, Chem. Mater, 15, pp. 1957-1962, 2003.

2) R.G. Rayavarapu, W. Petersen, C. Ungureanu, J.N. Post, T.G. van Leeuwen and S. Manohar, IJBI, 29817, 2007.

3) P.K. Jain, X. Huang, I.H. El-Sayed, M.A. El-Sayed, Acc Chem Res., 1, pp. 1578-1586, 2008.

4) C.J. Murphy, T.K. Sau, A.M. Gole, C.J. Orendorff, J. Gao, L. Gou, S.E. Hunyadi and T. Li, J. Phys. Chem. B, 109, pp. 13857-13870, 2005.

5) M.Z. Liu, and P. Guyot-Sionnest, J. Phys. Chem. B, 109, pp. 22192-22200, 2005.

6) C.J. Orendorff, and C.J. Murphy, J. Phys. Chem. B., 110, pp. 3990-3994, 2006.

7) N. R. Jana, L. Gearheart and C. J. Murphy, Adv. Mater, 13, pp 1389-1393, 2001.

8) D.K. Smith and B.A. Korgel, Langmuir, 24, pp. 644-649, 2008.

9) T. Niidome, M. Yamagata, Y. Okamoto, Y. Akiyama, H. Takahashi, T. Kawano, Y. Katayama, Y. Niidome. J Control Release, 12, pp. 343-347, 2006.

10) S. Pierrat, I. Zins, A. Breivogel and C. Sönnichsen, Nano Lett., 7, pp. 259-263, 2007.

11) H. Takahashi, Y. Niidome, T. Niidome, K. Kaneko, H. Kawasaki and S. Yamada, Langmuir, 22, pp. 25,2006

12) C.J. Orendorff, T.M. Alam, D.Y. Sasaki, B.C. Bunker and J.A. Voigt, ACS. Nano., 3, pp. 971-983, 2009.

13) S. Manohar, A. Kharine, J. C. G. van Hespen, W. Steenbergen, and T. G. van Leeuwen, J. Biomed. Opt. 9, 1172, 2004

14) S. Manohar, S.E. Vaartjes, J.C.G. van Hespen, J.M. Klaase, F. M. van den Engh, W. Steenbergen, and T.G. van Leeuwen, Opt. Express 15, 12277, 2007.

15) H.F. Zhang, K. Maslov, G. Stoica, and L.H. Wang, Nature Biotechnol. 24, 848, 2006.

16) A.A. Bednov, E.V. Savateeva, and A.A. Oraevsky, Proc. SPIE 4960, 21, 2003. 
17) Conjugates of Peptides and Proteins to Polyethylene Glycols Margherita Morpurgo and Francesco M. Veronese Methods in Molecular Biology, 283:Bioconjugation Protocols: Strategies and Methods

18) J. Wang, R. Lv, J. Xu, D. Xu and H. Chen, Anal Bioanal Chem, 390, pp. 1059-1065, 2008.

19) C. Hamers-Casterman, T. Atarhouch, S. Muyldermans, G. Robinson, C. Hamers, E.B. Songa, N. Bendahman and R. Hamers, Nature., 363, 1993.

20) S.D. Perrault, C. Walkey, T. Jennings, H.C. Fischer and W.C.W. Chan, Nano Lett., 9, 2009. 


\section{Summary}

The thesis describes the use of gold nanorods as molecular probes for light-based imaging techniques for potential applications in biology and medicine. An overview of the synthesis and critical parameters influencing the synthesis of gold nanorods on size/shape/yield of nanorods are discussed. For using gold nanorods in vivo, the concerns of toxicity, biodistribution and bioconjugation are addressed to make them suited for use in vivo.

Chapter 1 introduced the literature overview on gold nanoparticles to be used in the applications of biomedicine. The chapter describes initially the potential of gold nanospheres and their advantages/disadvantages for in vivo biomedical applications. Gold nanospheres due to their lack of absorption frequencies in the NIR region are not suitable for deep in vivo imaging purposes in tissue due to absorption of light by hemoglobin and other chromophores and scattering by tissue constituents. To overcome this obstacle, we chose to synthesize gold nanorods that have the ability to absorb in the NIR region where light penetration in tissue is higher. The aims of the thesis and the objectives/goals to be achieved are briefly described.

Chapter 2 described the synthesis of gold nanorods that have tunable longitudinal plasmon peaks absorbing from far red to the near infrared (NIR) region. The gold nanorods synthesized have the range of absorbance from $600 \mathrm{~nm}$ - $850 \mathrm{~nm}$ that falls within the optical imaging/therapeutic window. To provide targeting ability to a certain disease site, the gold nanorods were bioconjugated with HER81 monoclonal antibody (mAb) via electrostatic and hydrophobic interaction where they can attach to the receptors of the tumor cell.

Chapter 3 described the role of surfactant in synthesizing gold nanorods. Certain products of hexadecyltrimethylammonium bromide (CTAB) from specific suppliers did not favor the synthesis of gold nanorods but yielded only gold nanospheres during the synthesis. The iodide impurities in CTAB are responsible for non-formation of gold nanorods. The results presented showed that the products of CTAB vary from "lot-to-lot" even within the same product number.

The conventionally synthesized gold nanorods are cylindrical shaped with hemispherical endcaps. However, we described in Chapter 4 that by changing the ascorbate-gold ion ratios 
the shapes of nanorods can be changed to either dog-bone or dumbbell or irregular shaped nanostructures. These nanostructures have different optical peaks and thus show that the increased addition of ascorbic acid during growth stage led to the formation of different shapes of nanostructures.

The important aspect of using nanorods in clinical applications is to address the concern of toxicity of the as-prepared gold nanorods. Chapter 5 shows results of the in vitro toxicity of gold nanorods on four different cell lines (SKBR3, CHO, C2C12 and HL60) before /after polymer treatment. The CTAB capped gold nanorods are toxic to cells, PSS coated nanorods are less toxic to cells and PEGylated gold nanorods are the ultimate candidates that are nontoxic to cells and are biocompatible.

It is essential to know the fate of gold nanorods in vivo after injection in the tail vein of rat. The results described in Chapter 6 shows the biodistribution of gold nanorods in several organs (liver, spleen, lung, kidney, heart, brain, testes, thymus and blood) of rat. The CTAB capped gold nanorods disappeared within $\sim 10$ minutes of injection from the blood and were accumulated mostly in liver followed by spleen. The PEGylated gold nanorods are stable in blood circulation for longer than two days. The PEGylated gold nanorods were accumulated more in spleen compared to liver. This shows that the PEGylated gold nanorods are stable in blood stream and can be used in imaging and therapy.

As described in Chapter 2, the conjugation of gold nanorods was performed non-covalently. The non-covalent coupling of gold nanorods to antibodies is weaker and to overcome this obstacle we have used covalent mode of bioconjugating gold nanorods using PEG linkers as described in Chapter 7. The antibody conjugated gold nanorods are uptaken by SKBR3 cells with longer incubation periods via receptor-mediated endocytosis (RME). The immunostaining of gold nanorods showed successful coupling of gold nanorods to SKBR3 cells. The antibody conjugated gold nanorods toxicity was evaluated using propidium iodide (PI) assay and they are not toxic to cells even though the conjugated gold nanorods are uptaken.

In Chapter 8 the results of the thesis are discussed, and outlook sketched with several possible improvements proposed. We conclude that functionalized gold nanorods are ideal for using them as molecular probes for light-based imaging techniques. 


\section{Samenvatting}

Dit proefschrift beschrijft het gebruik van goudnanostaafjes als moleculaire markers voor beeldvorming in biologische en medische toepassingen. Er wordt een overzicht gegeven van de synthese van de goudnanostaafjes, alsmede van de parameters die tijdens deze synthese de vorm, grootte en opbrengst van de nanostaafjes beïnvloeden. Om de goudnanostaafjes geschikt te maken voor gebruik in vivo, worden ook aspecten als toxiciteit, biodistributie en bioconjugatie onderzocht.

In Hoofdstuk 1 wordt een literatuuroverzicht van het gebruik van goudnanodeeltjes in biomedische disciplines gegeven. Dit hoofdstuk start met een beschrijving van de potentie van goudnanobolletjes en hun voor- en nadelen voor in vivo biomedische toepassingen. Goudnanobolletjes vertonen weinig absorptie van nabij infrarood (NIR) licht. Dit maakt de goudnanobolletjes niet geschikt voor diepe in vivo beeldvorming in weefsel aangezien licht van andere golflengtes sterk wordt geabsorbeerd en verstrooid in weefsel. Om deze beperking te overwinnen, hebben wij ervoor gekozen om goudnanostaafjes te synthetiseren. De goudnanostaafjes hebben wél de eigenschap om het NIR licht, dat diep in weefsel door kan dringen, te absorberen. In dit hoofdstuk worden de doelstellingen van het proefschrift kort beschreven.

Hoofdstuk 2 beschrijft de synthese van goudnanostaafjes die een afstelbare longitudinale plasmonpiek hebben en licht absorberen van het uiterst zichtbare tot het NIR gebied. De gesynthetiseerde goudnanostaafjes absorberen licht tussen $600 \mathrm{~nm}$ en $850 \mathrm{~nm}$ en vertonen daarmee absorptie in het zogenaamde therapeutische of optische beeldvormingsvenster. Om de nanostaafjes specifiek te kunnen richten op een eventuele maligniteit, worden ze gebioconjugeerd met HER81 monoclonale antilichamen (mAB) via electrostatische en hydrofobische interacties. Dit maakt binding aan de receptoren van de tumorcel mogelijk.

Hoofdstuk 3 beschrijft de rol van surfactant in de synthese van de goudnanostaafjes. Met een bepaald product van hexadecyltrimethylammonium bromide (CTAB) van een zekere fabrikant was het niet mogelijk om goudnanostaafjes te synthetiseren, maar konden alleen goudnanobolletjes worden verkregen. Jodium verontreinigingen in de CTAB waren de oorzaak van het feit dat er geen goudnanostaafjes konden worden gevormd. De resultaten 
laten zien dat de samenstelling van CTAB producten kan variëren tussen eenheden met eenzelfde productnummer.

De conventioneel gesynthetiseerde goudnanostaafjes zijn cilinder-vormig met halfronde uiteinden. In Hoofdstuk 4 wordt beschreven dat door de ratio tussen ascorbine en goud ionen te veranderen, de vorm van de nanodeeltjes kan worden veranderd in botvormig, haltervormig of onregelmatig gevormd. Deze verschillende nanostructuren vertonen verschillende optische absorptiepieken. Het toevoegen van een grotere hoeveelheid ascorbinezuur tijdens de groeifase van de nanodeeltjes leidt dus tot de synthese van verschillende soorten nanodeeltjes.

Een belangrijk aspect met betrekking tot het gebruik van de goudnanostaafjes in klinische toepassingen is hun eventuele toxiciteit. In Hoofdstuk 5 staat het resultaat van de studie naar de in vitro toxiciteit van goudnanostaafjes op vier verschillende cellijnen (SKBR3, CHO, C2C12 en HL60) voor en na polymeer behandeling. De CTAB gestabiliseerde goudnanostaafjes zijn toxisch voor cellen, PSS gestabiliseerde goudnanostaafjes zijn minder toxisch en PEG gestabiliseerde goudnanostaafjes zijn de ultieme kandidaat voor klinische toepassingen: ze zijn niet toxisch voor cellen en ze zijn biocompatibel.

Het is essentieel om het lot van de goudnanostaafjes in vivo te kennen na injectie. De resultaten die worden beschreven in Hoofdstuk 6 laten de biodistributie van de goudnanodeeltjes in verschillende organen (lever, milt, longen, nieren, hersenen, testes, thymus en bloed) van ratten zien, na injectie van de staafjes in hun staartvenen. De CTAB gestabiliseerde goudnanostaafjes verdwenen binnen $~ 10$ minuten na injectie uit het bloed en accumuleerden voor het grootste gedeelte in de lever, gevolgd door de milt. De PEG gestabiliseerde goudnanostaafjes bleven langer dan twee dagen in de bloedcirculatie en accumuleerden voor een groter gedeelte in de milt dan in de lever. Dit laat zien dat de PEG gestabiliseerde goudnanostaafjes stabiel in het bloed blijven en dat ze kunnen worden gebruikt voor beeldvorming en therapie.

Zoals in Hoofdstuk 2 is beschreven, vindt de conjugatie van de goudnanostaafjes plaats via non-covalente bindingen. Een non-covalente binding van goudnanostaafjes aan antilichamen is zwakker dan een covalente binding. Om op deze beperking in te spelen, hebben we een covalente manier voor bioconjugatie van goudnanostaafjes toegepast met het gebruik van PEG linkers. Dit wordt in Hoofdstuk 7 besproken. De antilichaam geconjugeerde goudnanostaafjes worden met langere incubatieperioden door de SKBR3 cellen opgenomen 
via het proces van recepter gemedieerde endocytose. De immunokleuring van de goudnanostaafjes laat zien dat er succesvolle aanhechting van de goudnanostaafjes aan de SKBR3 cellen plaatsvindt. De toxiciteit van de antilichaam geconjugeerde goudnanostaafjes is beoordeeld met behulp van een propidium jodide analyse. Ondanks het feit dat de geconjugeerde goudnanodeeltjes worden opgenomen door de cellen, blijken ze niet toxisch te zijn.

In Hoofdstuk 8 worden de resultaten samengevat en bediscussieerd. Ook wordt een aantal mogelijke verbeteringen voorgesteld. We concluderen dat gefunctionaliseerde goudnanodeeltjes ideaal zijn voor gebruik als moleculaire markers in verschillende biomedische toepassingen, zoals bijvoorbeeld beeldvormende technieken met licht. 


\section{Abbreviations}

GNR, AuNR- gold nanorods

GNP- gold nanoparticle

GNS- gold nanosphere

CTAB- hexadecyltrimethylammonium bromide

PEG- polyetheylene glycol

PSS- polystyrene sulfonate

AA- ascorbic acid

$\mathrm{AgNO}_{3}$ - silver nitrate

OPSS-PEG-NHS- ortho-pyridyl disulfide-PEG-Succinimidyl Ester

$\mathrm{NaBH}_{4}$ - sodium borohydride

$\mathrm{HAuCl}_{4}$ - choloroauric acid

cGNR- conjugated gold nanorods

mAb- monoclonal antibody

EDTA- ethylenediaminetetra acetate

PBS- phosphate buffer saline

SEM- scanning electron microscope

TEM- transmission electron microscope

ZP- zeta potential

mPEG-SH- methoxy(polyethylene glycol)-thiol

pM- picoMolar

LC- lethal concentration 


\section{Acknowledgements}

It is now time to thank all the people who have directly or indirectly been associated with my research during the four years of my PhD study.

I thank my promotor Prof. Ton van Leeuwen for allowing me to work on this project. I thank him for his constant support and encouragement during all these four years. Ton, I express my sincere gratitude to you for giving me an extension that helped me finish writing my thesis. You were very kind and friendly throughout these years and encouraged me at every step of my PhD. Thank you for all the fruitful discussions during all these years of my $\mathrm{PhD}$.

I thank Dr. Srirang Manohar, my assistant promotor, for showing faith in me and considering me for this $\mathrm{PhD}$ position. I have enjoyed working with you all these four years and coming to this stage of my $\mathrm{PhD}$ having a thesis to defend is only because of your invaluable suggestions, critical comments, weekly meetings and your constant guidance throughout the research. Whenever I have doubts during the project, you were always there to teach and correct me. I truly appreciate your meticulous corrections on all my scientific outputs resulted from this work. All that I have learned from you during these four years will help me very much in my academic career in the future. Srirang, not only am I amazed by your scientific skills, your skills in photography too are excellent.

I thank Prof. Vinod Subramaniam for allowing me to join the group (BPE) as a PhD student. I thank you for your suggestions regarding certain aspects of the project especially for initiating the collaboration with RIVM for the biodistribution studies of gold nanoparticles.

Wilma, your technical support during all these years is invaluable and without your help, the chapters of toxicity and bioconjugation studies would not have been possible. I thank you for your contribution. Constantin, my friend, all the years working together with you made me learn many things related to physics. I enjoyed the lunches and dinners with you along with Felicia and the small scientist (Gabriel). The work in the thesis would not have been possible without your inputs on nanoparticles. I acknowledge the initial contribution in making gold nanoparticles by Sam Mathew, Christina Graf for her inputs on glassware cleaning using hydrofluoric acid. I thank all the people in the NIMTIK project for the useful meetings and discussions.

I thank Dr. Wiendelt Steenbergen for organizing the weekly work meetings that has helped me very much in improving my presentation skills. I thank Prof. Leon Terstappen for providing the HER81 monoclonal antibody for my research. I thank Dr. Cees Otto and Henk-Jan van Manen for their initial help in characterizing gold nanoparticles using the Raman set-up. I thank Tom Groothuis for the discussions on cellular uptake of nanoparticles. Dodo, you are friendly and helpful person. I thank you 
for organizing the useful meetings of Gold Mafia. I would like to thank Remco and Liesbeth for the useful discussions on nanoparticles.

I thank Severine le Gac for her help with the initiation of toxicity studies on gold nanoparticles, which led to in vitro and in vivo studies. I thank Dr. W. De Jong (RIVM) for his collaboration, which led to chapter 6 of my thesis. I thank Petra Krystek (MiPlaza, Philips Research) for the ICP-MS measurements. I thank Dr. Fijs van Leeuwen, Patrick Chin and Hans Janssen (NKI) for their collaboration in using TEM studies on cells with nanoparticles. I thank Prof. Johan Engbersen (PBM) for discussions regarding bioconjugation of gold nanorods using the PEG linker. I thank Mark Smithers who helped me with visualization of gold nanoparticles using SEM/TEM. I thank Wei Zhou (PBM) for teaching me how to use the Zetasizer.

Many thanks to cheerful Sylvia, who always smilingly helped me with the bureaucratic work. I thank Johan, Robert, Erwin, Christian for their help in solving the problems of my office computer. I thank Kirsten, Yvonne and Marloes for their help in the chemical lab and ordering of chemicals.

I like to thank my office-mates during the years, Kiran, Dianwen, Arjen, Gertjan, Wenfeng, Yujiye Ma. Michelle Heijblom, thank you so much for helping me with the Dutch translation of my summary. I thank Chien-Ching, Alex, Daniele, Cicerone, Ine-Segers and Roy Kolkman for their friendly nature. I thank my desi friends- Ganeshram, Ram, Sandeep, Babu, Kiran, Vinay and Chandra for their friendly talks.

Vishnu, thank you for your friendship and for your help whenever I required it. Not only are you an excellent researcher, you are good cook too. All the dinners with you and Anand Chakinala have been delicious. I like to thank my other two good friends Jithin Jose and Shashank Shekhar who always generously shared their lunch boxes with me. Renuka, Aruna and Hari thanks for always being there, in good as well as the bad times of my life. I cherish our long friendship.

Finally and most importantly, I owe my gratitude to my parents (Suryanarayana and Kasulamma) and brother (Hemanth Kumar) who encouraged me with their caring, love and unconditional support all through the years. I dedicate my thesis to my parents, brother and my teachers who taught me throughout my academic career.

Raja Gopal Rayavarapu

Enschede, March 2010. 


\section{List of Publications}

\section{Journal articles (peer-reviewed)}

R. G. Rayavarapu, C. Ungureanu, T. G. van Leeuwen and S. Manohar; "Iodide impurities in hexadecyltrimethylammonium bromide (CTAB) products: lot-lot variations and influence on gold nanorod synthesis”, Langmuir, DOI: 10.1021/la 100166f, 2010.

R. G. Rayavarapu, W. Petersen, L. Hartsuiker, P. Chin, H. Janssen, F. W. B. van Leeuwen, C. Otto, S. Manohar and T. G. van Leeuwen, "In vitro toxicity of polymer coated gold nanorods", Nanotechnology, 2010 (in print).

R. G. Rayavarapu, W. Petersen, C. Ungureanu, J. Post, T. G. van Leeuwen and S. Manohar, "Synthesis and Bioconjugation of gold nanoparticles as potential contrast agents for light-based imaging techniques”, Intl. J. Biomed. Imaging, 29817, 2007.

C. Ungureanu, R. G. Rayavarapu, S. Manohar and T. G. van Leeuwen, "Discrete Dipole Approximation simulations of gold nanorod optical properties -Choice of input parameters and comparison with experiment”, J. Appl. Phys. 105, 102302, 2009.

\section{Articles under review}

R. G. Rayavarapu, T. G. van Leeuwen and S. Manohar, "Maneuvering gold deposition to tailor the tips of gold nanorods by changing initial ascorbate-gold ion ratios in synthesis".

D. P. K. Lankveld, R. G. Rayavarapu, P. Krystek, A. G. Oomen, H. W. Verharen, T. G. van Leeuwen, W. H. De Jong and S. Manohar, "Blood clearance and tissue distribution of PEGylated and Non-PEGylated gold nanorods after intravenous administration in rats”.

C. Ungureanu, A. Amelink, R. G. Rayavarapu, H. J. C. M. Sterenborg, S. Manohar and T. G. van Leeuwen”, Differential pathlength spectroscopy for the quantitation of optical properties of gold nanoparticles".

C. Ungureanu, R. Kroes, Y. Candel, R. G. Rayavarapu, A. Poot, S. Manohar and T. G. van Leeuwen, "Hyperthermia using gold nanorods: A simulation study".

\section{Manuscripts in preparation}

R. G. Rayavarapu, W. Petersen, H. Janssen, P. Chin, F. W. B van Leeuwen, T. G van Leeuwen and S. Manohar, "Antibody conjugation to gold nanorods via PEG linkers: in vitro toxicity and cellular uptake studies in breast adenocarcinoma cell line".

C. Ungureanu, R. Kroes, R. G. Rayavarapu, S. Manohar and T. G van Leeuwen, "Photothermal destruction of gold nanorods”.

C. Ungureanu, R. Kroes, R. G. Rayavarapu, W. Petersen, S. Manohar and T. G. van Leeuwen, "Photo thermal killing of tumor cells using gold nanorods". 
L. Hartsuiker, W. Petersen, R. G. Rayavarapu, S. Manohar, T. G. van Leeuwen, L. W. M. M. Terstappen and C. Otto, "Fluorescent-like broadband Raman response of gold nanorods in live breast cancer cells”. 


\section{About the author}

Raja Gopal Rayavarapu was born in Pentasriramapuram, Andhra Pradesh, India on October 5, 1978. He obtained his Bachelor of Science (B.Sc) degree in Biochemistry from P.B. Siddhartha College of Arts and Sciences, Nagarjuna University, India. In 2001, he completed his Masters of Science (M.Sc) degree in Biochemistry from Nagarjuna University, Andhra Pradesh, India. From 2001 until 2002, he worked as a teacher for biological sciences in R. J. College, Rajahmundry, India.

From January 2003 until September 2003, he worked as a technician in National Brain Research Center (Department of Biotechnology, CSIR), Government of India. In 2005, he achieved another Masters of Science (M.Sc) degree in Molecular Bioengineering from Technical University Dresden (Germany). During his Masters, he carried out a research project in BNS group in the supervision of Dr. Micheal Mertig, Prof. Daniel Mueller and Prof. Wolfgang Pompe, titled "Construction of low-dimensional hexagonal DNA networks for biosensor applications”.

From November 2005 until March 2010, he performed research towards his PhD thesis titled as "Gold nanorods as molecular probes for light-based imaging techniques" at the Biophysical Engineering Group (BPE) of the University of Twente, The Netherlands under the supervision of Prof. Dr. Ton G. van Leeuwen and Dr. Srirang Manohar. The thesis is focused on synthesis, functionalization, characterization and studying the biocompatibility of gold nanorods for both in vitro as well as in vivo studies 
